\title{
Morphological Analysis of Kenyon Cells of the Drosophila Mushroom Bodies
}

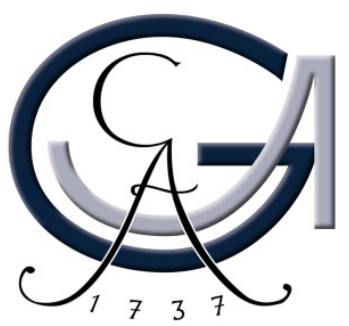

Dissertation

for the award of the degree

“Doctor rerum naturalium"

of the Georg-August-Universität Göttingen

submitted by
David Vasmer

From

Bremen, Germany

Göttingen, 2016 


\section{PhD Committee Member:}

Prof. Dr. André Fiala (Supervisor, Reviewer)

Dept. of Molecular Neurobiology of Behavior

Johann-Friedrich-Blumenbach Institute for Zoology and Anthropology

Georg-August-University, Göttingen

\section{PhD Committee Member:}

Prof. Dr. Martin Göpfert (Reviewer)

Dept. of Cellular Neurobiology

Johann-Friedrich-Blumenbach Institute for Zoology and Anthropology

Georg-August-University, Göttingen

\section{PhD Committee Member:}

Camin Dean, PhD

Dept. of Trans-synaptic Signaling

European Neuroscience Institute, Göttingen

Thesis submission: November 8, 2016 


\section{Declaration of academic honesty:}

I hereby declare that the doctoral thesis entitled "Morphological Analysis of Single

Kenyon Cells of the Drosphila Mushroom Bodies" has been written independently and with no other sources and aids other than quoted within texts, references and acknowledgements.

David Vasmer

Göttingen, November 8, 2016 


\section{Table of contents}

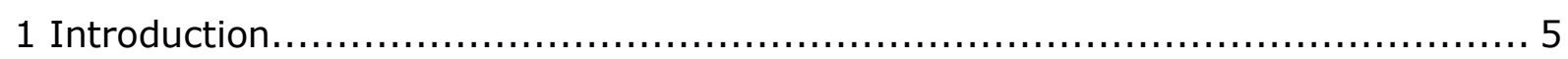

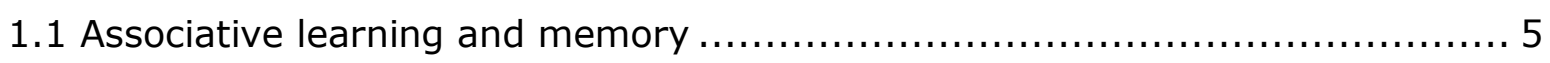

1.2 Learning and memory in Drosophila............................................ 6

1.3 Genetic tools for the study of learning and memory in Drosophila ............... 7

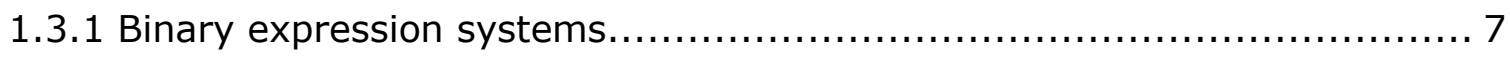

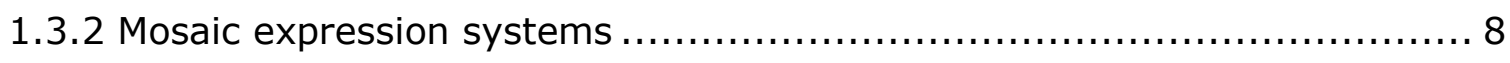

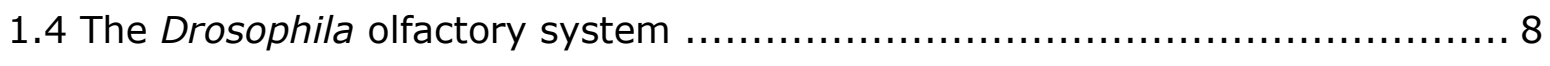

1.5 The molecular basis of olfactory associative memory in Drosophila............. 13

1.6 The role of the mushroom bodies in Drosophila olfactory associative learning

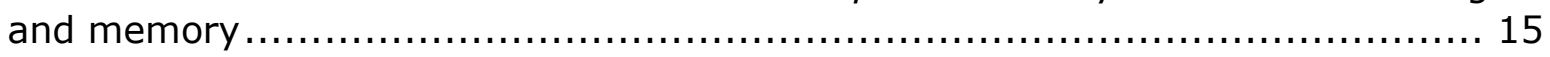

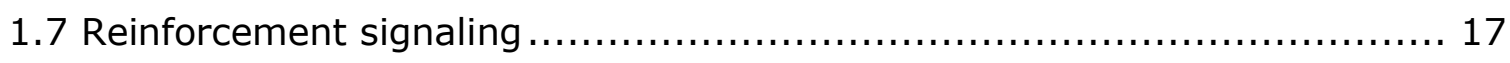

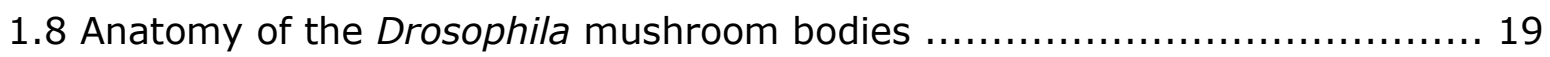

1.8.1 Morphological organization of the Drosophila mushroom bodies ........... 19

1.8.2 Organization of mushroom body extrinsic neurons ...................... 22

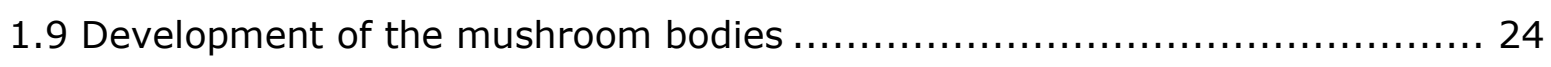

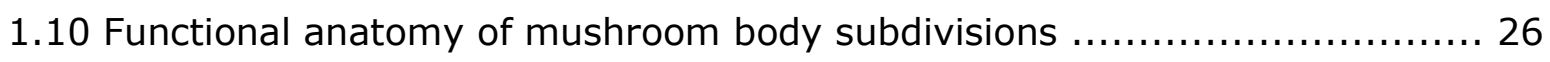

1.10.1 Functional anatomy of Kenyon cell classes ........................... 26

1.10.2 Functional anatomy of mushroom body compartments ................. 28

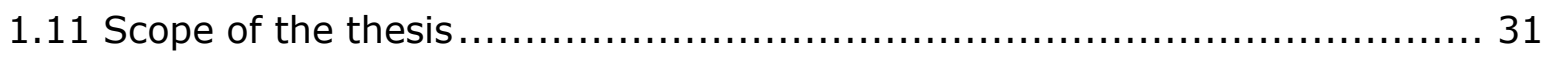

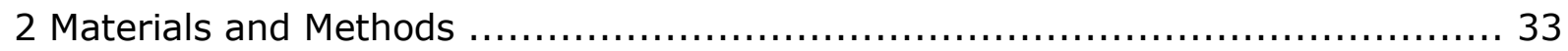

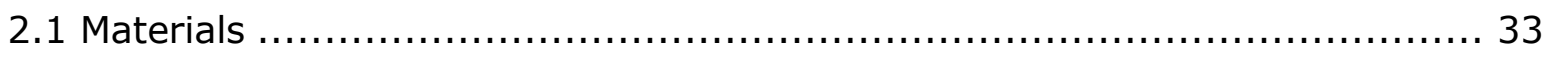

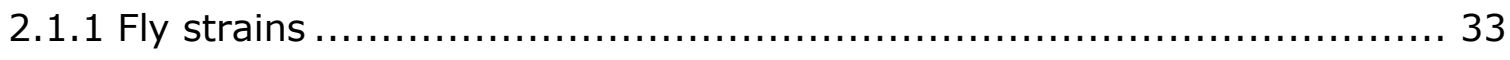

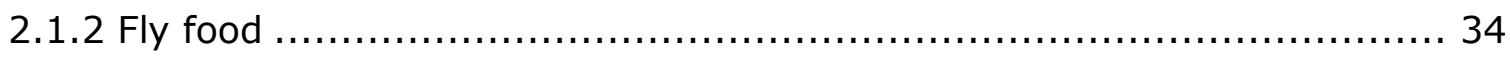

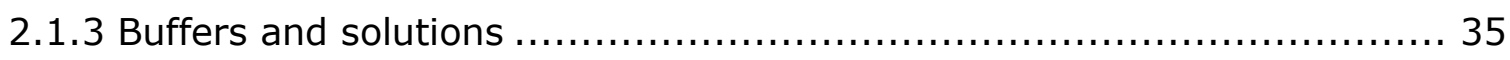

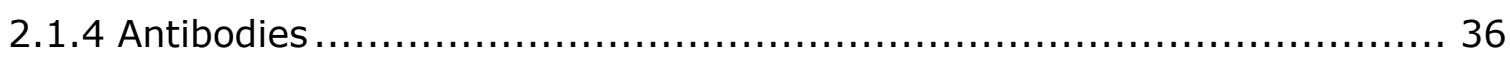

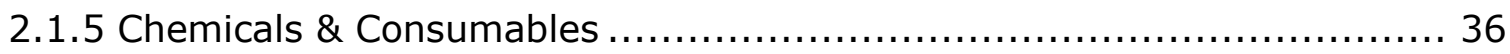

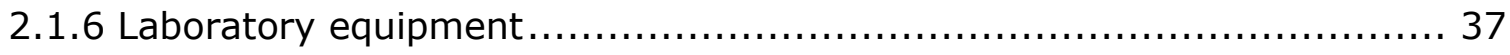

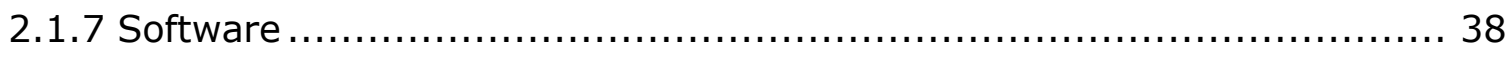

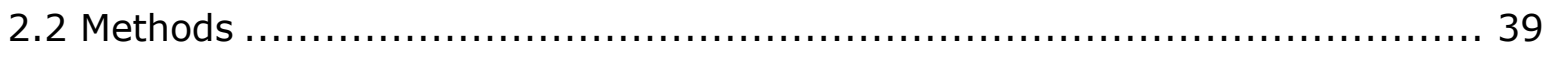

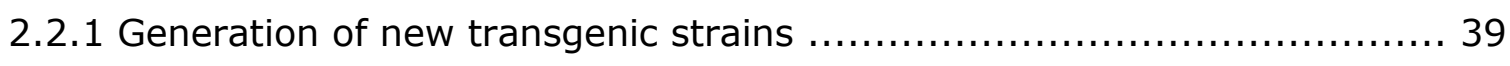

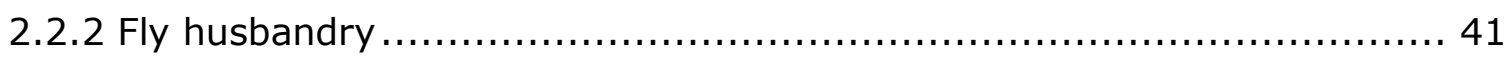

2.2.3 MARCM dependent single cell clone generation by heat shock ............. 42

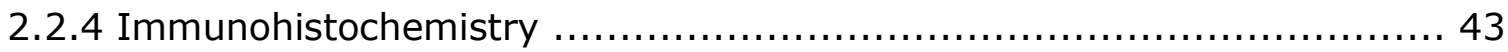

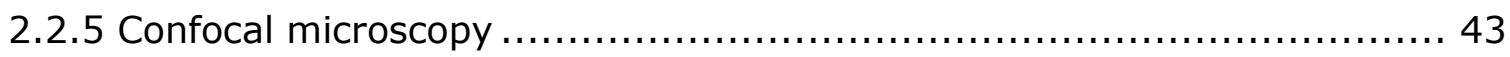

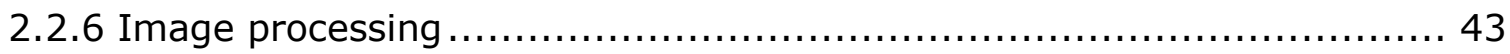




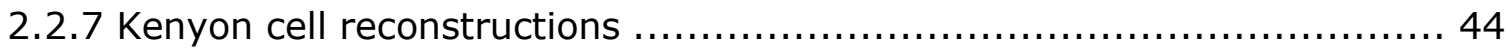

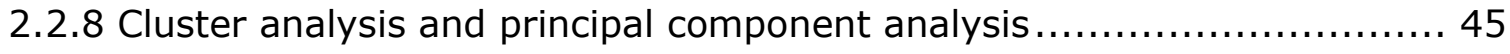

2.2 .9 Kenyon cell silhouettes................................................ 45

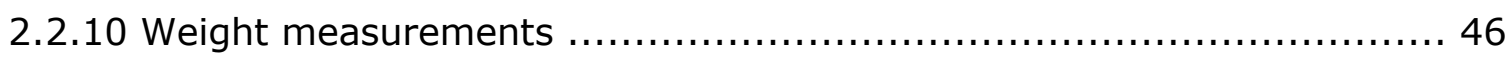

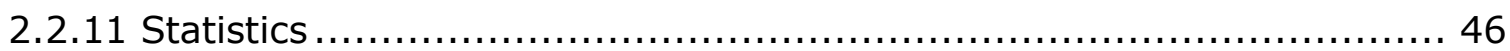

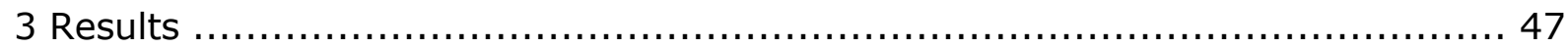

3.1 Establishing single cell anatomy of mushroom body Kenyon cells ............ 47

3.1.1 A Flp-out technique to label individual Kenyon cells ...................... 47

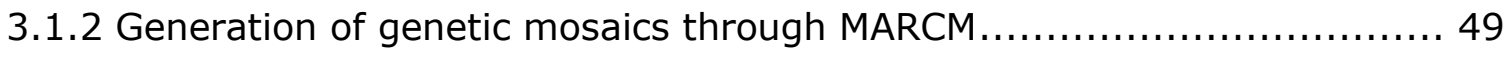

3.1.3 Digital reconstruction of individual Kenyon cells .......................... 54

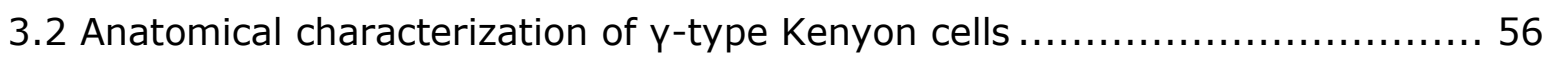

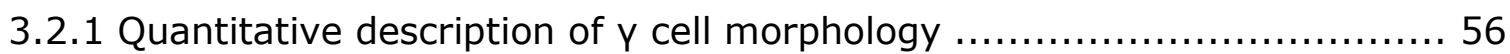

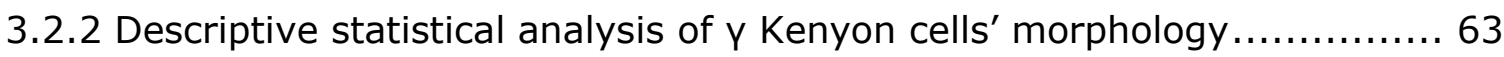

3.2.3 Quantitative description of Kenyon cell axonal arborizations $\ldots \ldots \ldots \ldots \ldots \ldots \ldots \ldots \ldots$

3.2.4 Descriptive statistical analysis of y Kenyon cells' axonal arborizations .... 76

3.3 Analysis of structural plasticity of individual Kenyon cells ................... 80

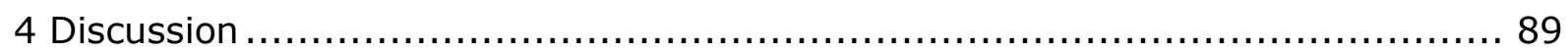

4.1 Single $y$ lobe Kenyon cells are morphologically diverse ..................... 89

4.2 Description of Kenyon cell subtypes........................................ 93

4.2.1 y Kenyon cell subtypes can be defined by axonal bifurcation............... 93

4.2.2 y Kenyon cell subtypes are identified by hierarchical cluster analysis .... 94

4.3 Nutritional value and structural plasticity of Kenyon cells ...................... 95

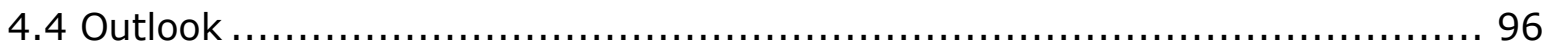

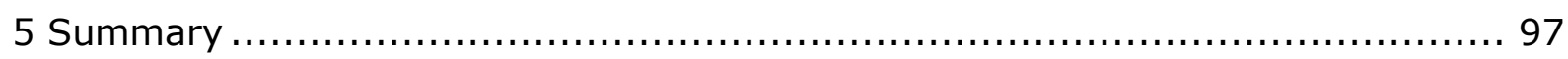

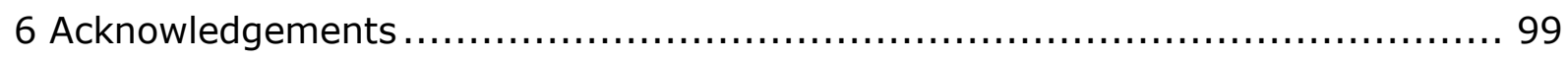

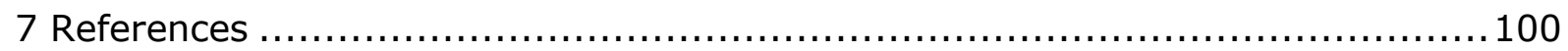

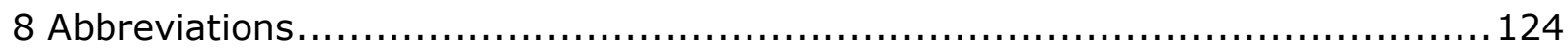

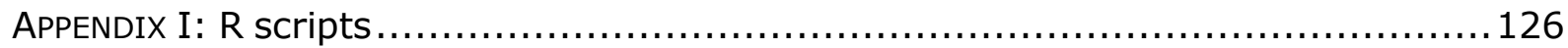




\section{Introduction}

\subsection{Associative learning and memory}

The mnemonic process of association has been described as early as $~ 300$ B.C. by Aristotle in his Parva Naturalia. Often, it is referred to the law of contiguity, which Aristotle stated as a memorization of things and events that are contiguous in time and space (Aristotle, trans. 1910; Buckingham and Finger, 1997). It was Ivan Pavlolv, who later addressed the subject of associative learning from an experimental perspective in his famous studies on classical conditioning with salivating dogs (Pavlov, 1906). In those experiments, the presentation of a food stimulus (the unconditioned stimulus, US) elicited an unconditioned response, that is, the dog started salivating. During training, the presentation of the food was repeatedly preceded by the ringing of a bell, the conditioned stimulus (CS). Subsequently, when subjected to only the sound of the bell, the dog also produced saliva, which is then called a conditioned response. In the following century, conditioned responses have been observed in a vast range of animal species and researchers have developed several model preparations in order to investigate associative learning in greater detail (reviewed in Schreurs, 1989). Well known model preparations are the siphon withdrawal reflex in Aplysia (Carew et al., 1981), the eyelid reflex in humans (Grant and Adams, 1944), spinal conditioning in the paralyzed cat (Patterson et al., 1973), and the nictitating membrane reflex in rabbits (Gormezano et al., 1962), just to mention a few. Phenomena of interest include memory acquisition, memory extinction, alpha response changes, and localization of memory in the central nervous system (Schreurs, 1989; Thompson, 1986), as well as operant learning (Thorndike, 1898). Nowadays, transgenic model organisms are widely used for the study of classical conditioning, and related phenomena, due to the many possibilities of labeling, and interfering with neurons (Kandel et al., 2014; Venken et al., 2011). Thus, the identification of a complete neural circuit behind associative memory has come within reach. 


\subsection{Learning and memory in Drosophila}

Drosophila has developed to an interesting model system in neuroscience because it combines a strong history of genetic research with an unexpectedly broad behavioral repertoire (reviewed for example in Anderson and Adolphs, 2014; Pitman et al., 2009; Sokolowski, 2001). Seymour Benzer and his students were pioneers in the field of Drosophila learning and memory in the 1970s. They could show that flies are able to associate an electric foot shock as an US with an odor stimulus as a CS, and further developed a paradigm to show that flies can discriminate between two colors when trained aversively with the bitter tastant quinine (Quinn et al., 1974). In subsequent experiments it was found that flies develop consolidated forms of olfactory memory, which are partly sensitive and partly resistant to cold shock anesthesia after training (Quinn and Dudai, 1976), and olfactory long-term memory traces were discovered, which last up to $24 \mathrm{hrs}$, and are dependent on novel protein synthesis (Tully et al., 1994). In contrast to appetitive olfactory long-term memory that was formed after a single training session (Tempel et al., 1983), aversive olfactory long-term memory formation required multiple training trials that had to be spaced in time (Tully et al., 1994). With the establishment of an efficient and well controlled training paradigm (Tully and Quinn, 1985), olfactory conditioning in Drosophila has become a major field of research for the study of the neural circuitry underlying associative memory. In this paradigm, groups of flies are trained aversively, using electric shocks as a punishment, to distinguish one reinforced odor (the CS+) against a non-reinforced odor (the CS-), and are subsequently tested in a T-maze choice assay.

Drosophilae are further able to perform in a great variety of visual learning tasks. They can learn to associate optic patterns with various aversive stimuli (Dill et al., 1993; Guo and Götz, 1997), to discriminate colors, shapes, and light intensities (Schnaitmann et al., 2013; Tang and Guo, 2001), and as for olfactory conditioning, they can form consolidated forms of visual memory (Xia et al., 1997). Most strikingly, flies can learn to navigate through a virtual maze based on landmarks (Ofstad et al., 2011). Apart from learning visual and olfactory cues, flies can be conditioned to alter the proboscis extension reflex (Brigui et al., 1990; Chabaud et al., 2006; Médioni and Vaysse, 1975), to avoid punished flavors (Masek and Scott, 2010), and to reduce courtship attempts towards females when they have experienced rejection before (Siegel and Hall, 1979). A good overview of Drosophila's abilities to perform in a total of 18 different visual learning, tactile, and motor learning tasks using different kinds of reinforcers is given in Wolf et al. (1998). Interestingly, flies are also able to 
generalize learned avoidance to stimuli that are perceived similarly (Barth et al., 2014; Ejima et al., 2005).

\subsection{Genetic tools for the study of learning and memory in Drosophila}

\subsubsection{Binary expression systems}

Binary expression systems have become the most commonly used tools in Drosophila neurogenetics, especially the Gal4/UAS system (Brand and Perrimon, 1993). The yeast transcription system allows for separation of the transcriptional activator (the transcription factor Gal4) and the effector protein (under control of the Gal4 specific upstream activating sequence [UAS]). Gal4 can be controlled directly by a cloned promoter region of interest or by the use of P-element insertions ( $O$ 'Kane and Gehring, 1987). P-element insertions link Gal4 expression randomly to a genomic promoter or enhancer, whose activity can then be visualized by an effector protein of choice - a technique known as enhancer trap. The target gene and the Gal4 driver are separated in two distinct transgenic fly lines. Any Gal4 driver line of interest can be crossed with a fly line carrying a UAS-controlled effector gene to generate flies in the F1 generation, in which the effector gene is expressed. Commonly used effector genes encode proteins for visualization of neurons such as GFP (Tsien, 1998) or RFP (Campbell et al., 2002), visualization of $\mathrm{Ca}^{2+}$ activity (Fiala et al., 2002; Tian et al., 2009), or transmitter release (Miesenböck et al., 1998), manipulation of neuronal activity by blockage of vesicle recycling (Kitamoto, 2001), light-gated depolarization (Nagel et al., 2003), or temperature induced activation (Hamada et al., 2008).

The Gal4/UAS system was further improved by the introduction of the Gal4 suppressor protein Gal80 (Lee and Luo, 1999; Ma and Ptashne, 1987) and its temperature sensitive version for spatiotemporal control of Gal4 expression (McGuire et al., 2003). Also the development of the split-Gal4 system, in which two parts of the Gal4 transcription factor are under control of separate promoters enabled researchers to target Gal4 expression to even smaller and more specific subsets of cells (Jenett et al., 2012; Luan et al., 2006). Other binary expression systems that can be used in combination with Gal4/UAS are QF/QUAS (Potter et al., 2010), LexA/lexAop (Lai and Lee, 2006), and FIp/FRT (Golic and Lindquist, 1989). For review see del Valle Rodríguez et al. (2012). 


\subsubsection{Mosaic expression systems}

In order to analyze subsets of cell types that cannot be labeled by binary expression systems or to label cell lineages, a number of mosaic expression systems have been developed. The Flp-out system (Golic and Lindquist, 1989; Harrison and Perrimon, 1993; Struhl and Basler, 1993) relies on excision of a stop cassette that is flanked by Flp recognition target (FRT) sequences. The stop cassette is located in front of a gene encoding a marker protein like lacZ or GFP, which is expressed after the excision event. Subsequent cell clones are likewise affected from the recombination and thus labeled as well. However, labeling of maturated cells is also possible. Induction of recombination by expression of Flp is usually achieved by heat shocking when Flp is under control of the hsp70 promoter (Ashburner and Bonner, 1979). An extension of the Flp-out system is the mosaic analysis with a repressible cell marker (MARCM, figure 1.1) (Lee and Luo, 1999). In MARCM, FRT sites are located at the same position in homologous chromosomes so that they potentially recombine during mitosis upon Flp activity. The ubiquitously expressed Gal4 repressor protein Gal80 is located on the same chromosome downstream of the FRT site. Animals are heterozygous for the Gal80 gene, wherefore following mitosis, one of the daughter cells will be homozygous for the repressor while in the other daughter cell and in its progeny Gal80 is absent. Combined with the Gal4/UAS system, this technique allows for the generation of single cell clones, in which Gal4 activity in a tissue of interest (dependent on the promoter that controls Gal4) is not suppressed by the Gal80 repressor leading to expression of the effector protein under UAS control. MARCM has widely replaced Golgi staining (Cajal and Sánchez, 1915) as a method for single cell labeling, and the efficiency of MARCM has even led to projects aiming to reconstruct the Drosophila brain at the single cell level (Chiang et al., 2011). Improvement of MARCM was achieved by twinspot MARCM, in which both daughter cells after mitosis and Flp induced recombination can be labeled in two different colors (Yu et al., 2009).

\subsection{The Drosophila olfactory system}

To understand the neural basis of olfactory associative memory an introduction to the Drosophila olfactory system is essential. Fruit flies have evolved a complex olfactory system that enables them to navigate through an odor-rich environment. They are able to localize food sources, to find mating partners, and to avoid dangers on the 

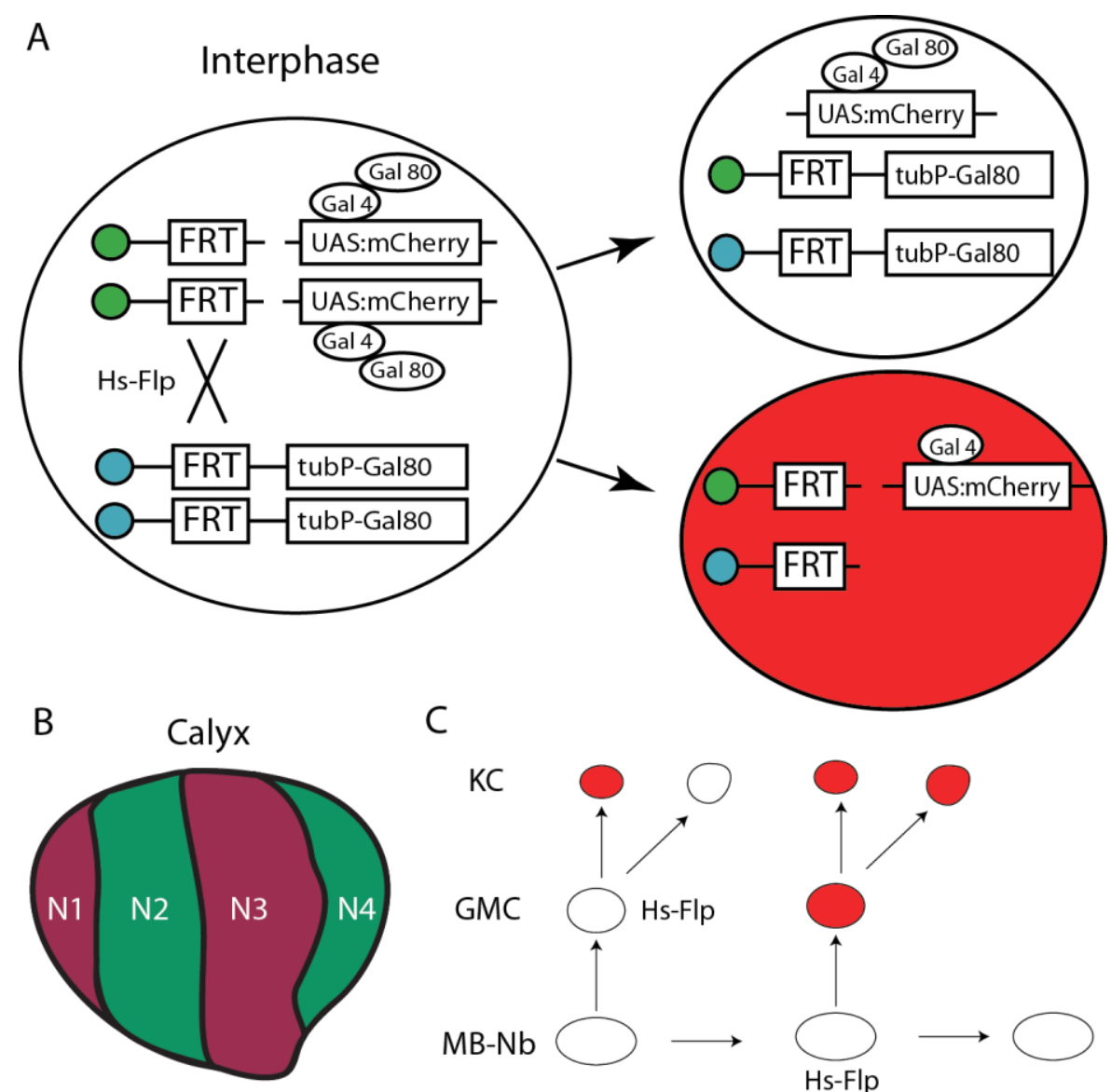

C

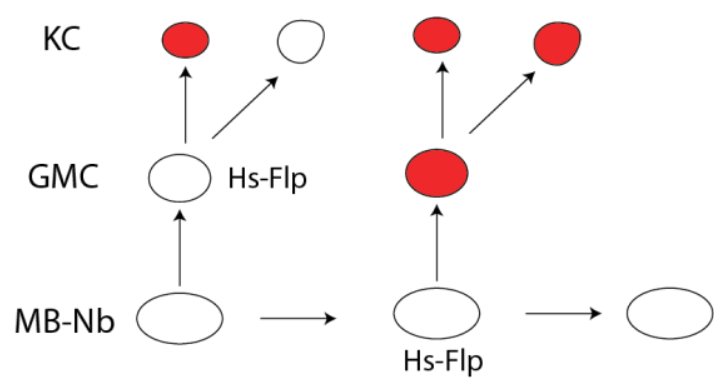

$\mathrm{D}$

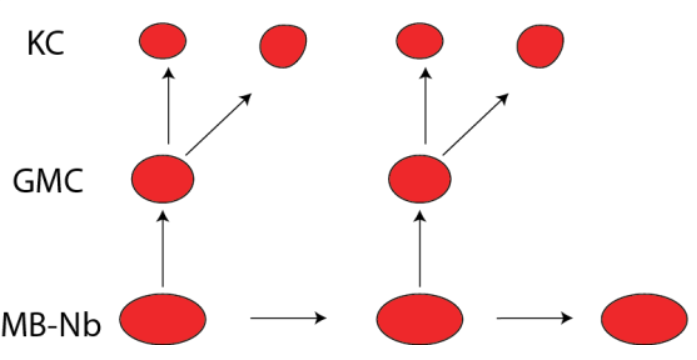

Figure 1.1. Mosaic analysis with a repressible cell marker (MARCM) illustrated on mushroom body Kenyon cells.

MARCM is a widely used genetic mosaic system for the analysis or labeling of single cell clones. It is based on induced recombination among homologous chromosomes during mitosis. In the present case, mCherry serves as a marker protein to visualize cell morphology.

A) Presumably during interphase after duplication of DNA strands has occurred, recombination can take place among homologous chromosomes induced by the activity of Flp recombinase at specific FRT sites. Flp is under control of a heatshock promoter, so that expression can be regulated by outside conditions. Cells are heterozygous in respect to the gene of interest, which is located distal to the recombination site. In MARCM, this gene, ubiquitously expressed via the tubulin promoter, codes for Gal80, a repressor of Gal4, which in turn can be driven in a tissue of choice. Upon recombination, successor cells can become homozygous for the Gal80 repressor, or completely lack the repressor. In the latter case, all successor cells will drive Gal4 expression and thus express the mCherry reporter. The UAS should be located on a different chromosome than the repressor in order not to be affected by the recombination event.

Figure 1.1 continued on next page. 
basis of olfactory cues. An overview of the Drosophila olfactory system is shown in figure 1.2 .

The detection of odors is achieved by olfactory receptors on the flies' antennae and maxillary palps. One to four different olfactory receptors are expressed within an olfactory receptor neuron, which in turn is localized to specialized sensillae belonging to four morphological classes: basiconic, coeloconic, trichoid, and palp basiconic (De Bruyne et al., 2001; Shanbhag et al., 1999). Canonical Drosophila olfactory receptors belong to an ancient diverse family of seven transmembrane proteins (Buck and Axel, 1991) and are encoded out of a set of 62 receptor genes (Robertson et al., 2003). For receptor functioning, co-expression of Or83b (also named olfactory co-receptor [Orco]) is essential (Larsson et al., 2004; Vosshall et al., 2000). The total amount of $\sim$ 1,300 olfactory receptor neurons per hemisphere are widely distributed over the antennae and palps. Interestingly, olfactory receptor neurons expressing the same kind of receptor converge in their projections to mostly one single glomerulus in the antennal lobe (Couto et al., 2005; Shanbhag et al., 1999; Vosshall et al., 2000). The antennal lobe consists of $\sim 54$ differently sized glomeruli arranged as spherical structures (Couto et al., 2005; Grabe et al., 2015). Each odor evokes spatiotemporal activity patterns of a set of olfactory receptor neurons that can be measured electrophysiologically, or using two-photon calcium imaging (Barth et al., 2014; Hallem and Carlson, 2006; Martelli et al., 2013; Wang et al., 2003). In addition, the antennal lobes are innervated by local interneurons of different morphologies ranging from unilateral uniglomerular to bilateral multiglomerular (Stocker et al., 1990). Those interneurons can be inhibitory or excitatory, and have been implicated in gain control, spatiotemporal shaping of odor identity, and in odor fine discrimination (Barth et al., 2014; Olsen and Wilson, 2008; Root et al., 2008; Shang et al., 2007; Wilson and Laurent, 2005).

Odor induced activity in the antennal lobe glomeruli is conveyed by about 200 olfactory projection neurons (PNs) to higher order brain centers via three different cerebral tracts (Marin et al., 2002; Stocker et al., 1990; Wong et al., 2002). The inner antennocerebral tract contains the largest number of PNs and is of particular interest

Figure 1.1 continued.

B) In the case of mushroom body development, four neuroblasts generate 95\% of Kenyon cells that dependent on the neuroblast (N1-N4) occupy distinct zones within in the mushroom body calyx.

C, D) Dividing mushroom body neuroblasts generate ganglion mother cells, each of which differentiates into two Kenyon cells. In dependence on when the Flp recombinase is active, and whether or not the daughter cell inherits the repressor, a single cell, or two cells (C), or the whole cell lineage (D) gets labeled by mCherry expression. Surely, all of the events can happen in parallel in different neuroblasts. Modified after Lee and Luo (1999). 


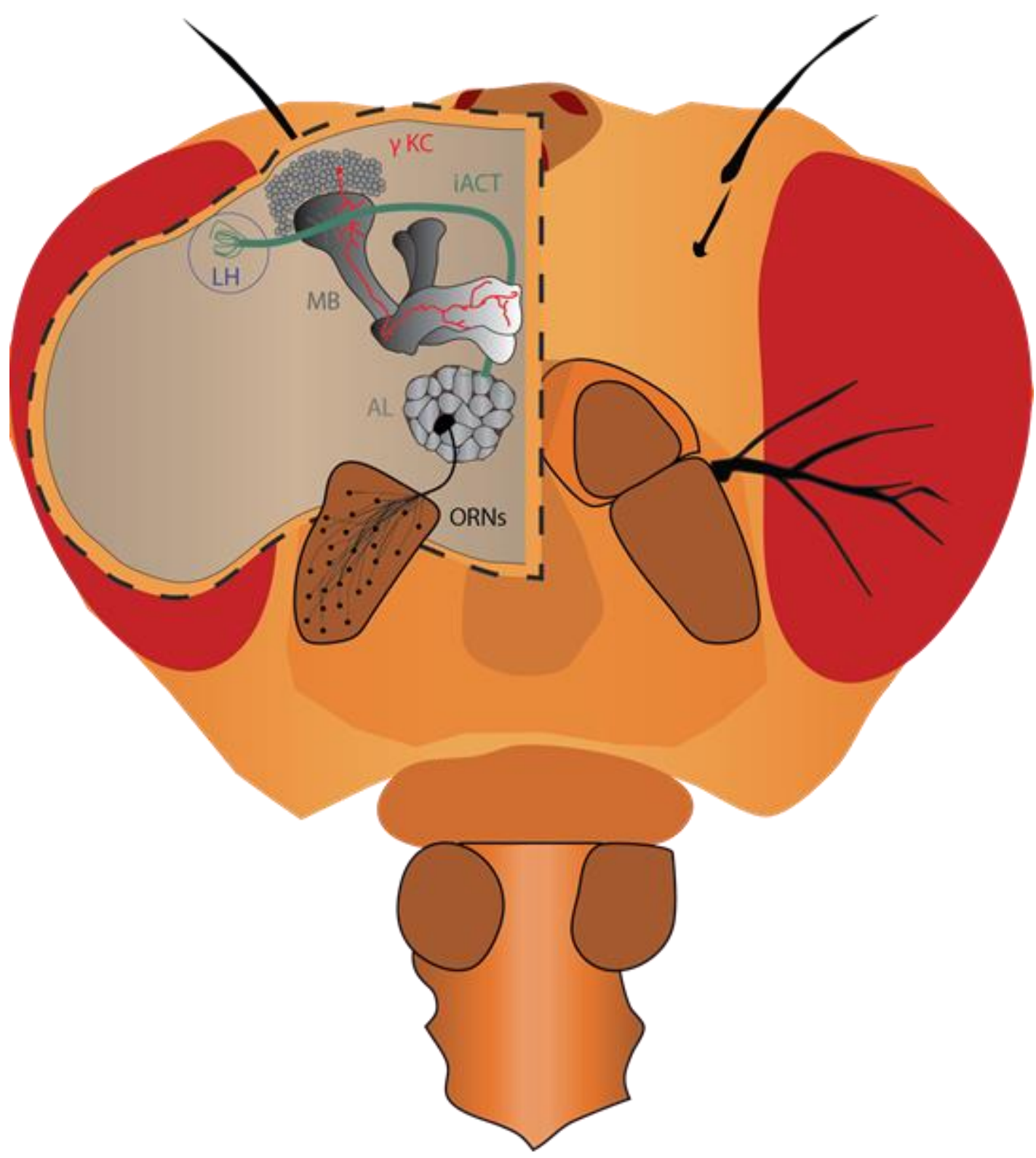

Figure 1.2. The Drosophila olfactory system.

Odor detection takes place on the flies antennae and maxillary palps, where odor molecules bind to olfactory receptors that are expressed in olfactory receptor neurons. Olfactory receptor neurons that express the same kind of receptor send axonal fibers to mostly one invariant glomerulus in the antennal lobe. Activity in glomeruli is regulated by inhibitory and excitatory local interneurons (not illustrated), and is taken up by dendrites of projection neurons that convey the signal mainly via the inner antennocerebral tract to higher order olfactory centers, namely the mushroom bodies and the lateral horn. Whereas the lateral horn is believed to mediate innate olfactory responses, Kenyon cells of the mushroom body have been shown to be necessary and sufficient for the support of associative memories. The cell bodies of Kenyon cells are located in a cup like shape around the calyx of the mushroom bodies, where Kenyon cell dendritic claws form connections to projection neuron boutons. Axonal Kenyon cell arbors project through the peduncle into the lobes of the mushroom body. Here, $a / \beta$ and $a^{\prime} / \beta^{\prime}$ neurons bifurcate to build the vertical $a / a^{\prime}$ and the horizontal $\beta / \beta^{\prime}$ lobes. The depicted $y$ neurons project only into the horizontal $y$ lobe. LH: lateral horn, MB: mushroom body, Y KC: Y Kenyon cell, iACT: inner antennocerebral tract, AL: antennal lobe, ORNs: olfactory receptor neurons. Modified after Heisenberg (2003). 
because the neurons form en passent synapses with dendritic arbors of Kenyon cells (KCs) in the mushroom body (MB) calyx before extending into the lateral horn (LH). The outer and medial antennocerebral tract are of smaller magnitude than the inner antennocerebral tract and constitute a direct connection from the antennal lobe to the lateral horn, while some fibers of the medial antennocerebral tract also send extensions to the MBs (Stocker et al., 1990). In contrast to the MBs that are involved in experience-dependent odor processing, the lateral horn is believed to mediate innate odor preferences (Gupta and Stopfer, 2012; Heimbeck et al., 2001; Heisenberg, 1998). It has been shown by mosaic studies that PNs projections are prespecified, in that they always innervate the same antennal lobe glomeruli and send stereotyped axonal fibers to the lateral horn and the MB calyx (Jefferis et al., 2001; Marin et al., 2002; Wong et al., 2002). Analysis of the output areas of olfactory PNs in the lateral horn using an image registration based technique showed that pheromone responsive PNs are spatially separated from fruit odor responsive PNs (Jefferis et al., 2007). In further experiments, the whole pheromone responsive circuitry could be dissected, from sensory input down to the ventral nerve cord and motor output areas (Datta et al., 2008; Ruta et al., 2010). Hard-wired behavioral programs were also found to be triggered by fruit odors like apple cider vinegar or by optogenetic activation of selected antennal lobe glomeruli (Bell and Wilson, 2016; Semmelhack and Wang, 2009).

In the calyx of the MBs, the acetylcholine producing PNs form connections to MB intrinsic KCs, and other extrinsic neurons that are partly GABA positive. Together, they compose the calycal microglomeruli, which are defined by one PN bouton and its postsynaptic partners (Leiss et al., 2009; Yasuyama et al., 2002). Stereotypy of PN $\mathrm{KC}$ connectivity, as it seems the case for hard-wired connections in the lateral horn, is subject of debate. On the one hand, it was found that the topography of identified PNs in the MB calyx is invariant across individuals, and that KCs of a particular type have stereotypic dendritic fields, which makes them likely to be connected to certain PN types (Jefferis et al., 2007; Lin et al., 2007). Furthermore, PNs that evoked responses in postsynaptic KCs were shown to be anatomically connected to more dendritic claws of these KCs than expected by chance (Gruntman and Turner, 2013). On the other hand, it could be shown by back-tracing the anatomical and functional input of single KCs to the respective PNs that neither PN nor KC identity was a reliable predictor for the potential synaptic partners (Caron et al., 2013).

Whereas PNs have a likelihood of $\sim 60 \%$ to respond to a given odor stimulus (Turner et al., 2008; Wilson et al., 2004), KCs show very low response rates lying around 5$10 \%$ in electrophysiological measurements (Murthy et al., 2008; Perez-Orive, 2002; 
Stopfer et al., 2003; Turner et al., 2008), and in $\mathrm{Ca}^{2+}$ imaging (Honegger et al., 2011). Thus, each odor is represented in terms of activity by 100-200 KCs of the overall population of $\sim 2,000 \mathrm{KCs}$ (Aso et al., 2009, 2014a) that comprise the Drosophila MB. The sparseness of the response is achieved by the high activation thresholds of $\mathrm{KCs}$, which integrate PN input across their dendritic claw population (Gruntman and Turner, 2013) and by feed-forward inhibition (Perez-Orive, 2002). Sparse coding is not a feature exclusively found in the insect MBs, but has also been described for neurons of the mammalian piriform cortex, which, interestingly, have likewise been implicated in learning and memory (Choi et al., 2011; Poo and Isaacson, 2009; Stettler and Axel, 2009). The advantage of sparse representations lies in the minimization of overlap between stimuli and thus reduced synaptic interference (Lin et al., 2014a; Masse et al., 2009; Turner et al., 2008).

Postsynaptic to KCs is a population of $\sim 35 \mathrm{MB}$ output neurons that are likely to convey $\mathrm{KC}$ responses to motor output areas. MB output neurons no longer represent odor identity but instead odor valence in order to guide appropriate behavioral responses. They have been shown to broadly respond to a given odor stimulus (Aso et al., 2014b; Hige et al., 2015a).

In summary, the Drosophila olfactory system has an interesting organization of convergence and divergence. At the first level of olfactory processing in the antennal lobe, the input of $\sim 1$,300 olfactory receptor neurons converges onto $\sim 54$ glomeruli or $\sim 200 \mathrm{PNs}$, enabling odor reception to be converted into an odortopic map, in which similar odors have similar topographics (Barth et al., 2014). At the next level, odor representations are again de-correlated within the set of $\sim 2,000 \mathrm{KCs}$, presumably to make each odor unique in its population activity pattern, thus maximizing information storage capacity, while saving energy (Olshausen and Field, 2004). In a last step, the $\sim 2,000 \mathrm{KCs}$ converge onto $35 \mathrm{MB}$ output neurons, which no longer encode the type of odor but its behavioral significance (Aso et al., 2014b).

\subsection{The molecular basis of olfactory associative memory in Drosophila}

The first studies on Drosophila learning mutants that fail to perform in olfactory conditioning enabled researchers to identify genes involved in memory formation. Research in Aplysia had already shown that the cAMP-PKA pathway is involved in shortand long-term sensitization of the gill-withdrawal reflex, and in the long-term form via 
the transcription factor CREB-1 (reviewed in Kandel, 2001). Interestingly, when forward genetics were used in Drosophila, the same pathway was found to be responsible for proper memory formation. The first described learning mutant selected from a screen of $~ 500$ mutated fly lines and found to be defective in olfactory associative learning was named dunce (Dudai et al., 1976), which encodes for a cAMP phosphodiesterase (Byers et al., 1981). Accordingly, dunce mutants were found to contain high levels of cAMP (Byers et al., 1981; Chen et al., 1986; Davis and Kiger, 1981). Another mutant fly line, named rutabaga, was found to have similar learning deficits as dunce. It encodes for a $\mathrm{Ca}^{2+} /$ calmodulin responsive adenylyl cyclase, and is thus required for the generation of CAMP (Levin et al., 1992; Livingstone et al., 1984), concluding that shortage as well as surplus of cAMP affects molecular memory formation pathways. Further experiments confirmed the role of the CAMP-PKA pathway in learning and memory in Drosophila. Flies mutated in $D C O$, the gene encoding for the catalytic subunit of PKA, or mutation of the gene encoding the regulatory PKA subunit dPKA-RI, showed reduced learning scores (Foster et al., 1988; Goodwin et al., 1997; Skoulakis et al., 1993). Using transgenes under the control of the heat shock inducible hsp70 promoter (Ashburner and Bonner, 1979) Drain et al (1991) inhibited PKA activity to interfere with the flies' learning ability and Griffith et al (1993) proved the necessity of $\mathrm{Ca}^{2+} /$ calmodulin-dependent kinase (CaMKII) in mnemonic processes. Another signaling pathway via the Ras-specific GTPase activating protein encoded by Neurofibromatosis-1 was identified to affect fly learning abilities presumably by disruption of the rutabaga mediated cAMP pathway (Guo et al., 2000).

CAMP seems to have various effects on memory formation. It was shown in Aplysia to activate the transcription factor CAMP response element-binding protein (CREB), which in turn regulates expression of proteins required for long-term facilitation of the gillwithdrawal reflex (Dash et al., 1990). Likewise in Drosophila, heat shock induced expression of a dominant negative form of $d$ CREB2 specifically inhibited long-term memory formation but not protein synthesis independent amnesia resistant memory (Yin et al., 1994). Short-term effects of CAMP-PKA include the phosphorylation of synaptic proteins (Diegelmann et al., 2006), and the manipulation of cyclic nucleotide gated ion-channels (Cheung et al., 2006; Pavot et al., 2015; Zhong et al., 1992). In sum, memory formation in Drosohila olfactory conditioning relies strongly on the CAMP-PKA pathway for alteration of gene expression and local synapse functioning. In accordance with the findings on synapse tagging and synaptic homeostasis (Redondo and Morris, 2011; Tononi and Cirelli, 2014), the identified molecular machinery might 
serve as a regulator for functional and structural plasticity of memory-relevant neurons.

\subsection{The role of the mushroom bodies in Drosophila olfactory associative learning and memory}

Analysis of Drosophila learning mutants not only led to the identification of genes that are necessary for memory formation, but also revealed several fly lines defective in olfactory learning with abnormally formed MBs (Heisenberg et al., 1985). The MBs are a central arthropod brain structure first described by Félix Dujardin (1850), who already proposed a role of the MBs in more complex behaviors (for review see Strausfeld et al., 1998). One learning mutant, named mushroom body deranged, lack the MB lobe structure, and is unable to perform in olfactory conditioning. Likewise do females of the mushroom body miniature mutant that completely lack the MBs. The males, in contrast, have normally developed MBs and show normal learning scores in olfactory associative learning (Heisenberg et al., 1985). More direct evidence for a role of the MBs in learning and memory came from honey bees in conditioning experiments, in which MB functionality was temporarily disturbed by local cooling (Erber et al., 1980; and see Hammer and Menzel [1995] for review). In Drosophila, it was further shown that feeding L1 larvae with hydroxyurea led to a selective deletion of the four neuroblasts that generate the MB intrinsic neurons (Ito and Hotta, 1992). Those flies showed normal odor and shock responsiveness but were unable to form a memory. Interestingly, in experiments, in which only partial ablation was observed, learning scores were significantly higher (de Belle and Heisenberg, 1994).

In the review article from 2004, Gerber and colleagues formulated several criteria for the identification of a memory trace in order to prove that olfactory memory is indeed localized to the MBs. They stated that neural plasticity has to occur in the cells of the alleged memory, that neural plasticity in these cells is necessary and sufficient for memory, that output from these cells is necessary for memory readout, and that input to these cells is necessary for memory acquisition (Gerber et al., 2004). Additionally, it should be possible to show that activity of the memory cells under observation is actually sufficient to elicit a behavioral response acquired by learning (Choi et al., 2011; Vasmer et al., 2014).

Evidence that neural plasticity occurs in the MBs due to learning is indicated by the investigated learning mutants: the phosphodiesterase encoded by dunce, the catalytic 
subunits of PKA encoded by $D C O$, and the adenylyl cyclase encoded by rutabaga are preferentially expressed in the MBs (Han et al., 1992; Nighorn et al., 1991; Skoulakis et al., 1993). It could further be shown, that genetic rescue of rutabaga in the MBs was sufficient to restore the memory deficits seen in the rutabaga mutant (Zars, 2000). Temporal control of the rutabaga rescue to adulthood substantiated the idea that the adenylyl cyclase encoded by rutabaga is directly required for learning and not just for proper MB development (Mao et al., 2004; McGuire et al., 2003). Thus, if it is agreed upon the role of CAMP in mediating neuronal plasticity (Kandel, 2001; Lee, 2015), the necessity and sufficiency of neural plasticity in the MBs is given.

The direct observation of neural plasticity in KCs has become possible with the development of new transgenic tools to image $\mathrm{Ca}^{2+}$ fluxes (Akerboom et al., 2012; Fiala et al., 2002; Pech et al., 2013a; Tian et al., 2009), PKA dynamics (Gervasi et al., 2010), or cAMP activity (Lissandron et al., 2007; Nikolaev et al., 2004; Shafer et al., 2008). Thereafter, a number of memory traces were identified as changes in post/pre training responses after paired olfactory conditioning in spatially restricted $\mathrm{MB}$ subregions and dependent on the time of test (Akalal et al., 2010, 2011; Barth et al., 2014; Boto et al., 2014; Tomchik and Davis, 2009; Wang et al., 2008; Yu et al., 2006). The requirement of $\mathrm{KC}$ activity for memory retrieval was shown using the temperature sensitive dominant allele of shibire (van der Bliek and Meyerowitz, 1991; Kitamoto, 2001) coding for the GTPase dynamin that is involved in endocytosis of synaptic vesicles. Temperature sensitive shibire becomes inactive at higher temperatures, thereby inhibiting synaptic transmission. Selectively inhibiting synaptic transmission of KCs by testing olfactory conditioned flies at restrictive temperatures, abolished memory retention (Dubnau et al., 2001; McGuire, 2001). Furthermore, extinction of the memory trace by repeated odor $(\mathrm{CS}+)$ exposures after training was not affected by inhibition of synaptic output (Schwaerzel et al., 2002). Blocking KC input during learning is until now not feasible due to the lack of appropriate tools that allow to specifically block PN output to the MBs but leave PN output to the lateral horn intact. However, me and my colleagues could show in previous experiments that sensory input to the MBs is not necessary for the formation of an associative memory (Vasmer et al., 2014). In these experiments, an mCherry tagged version of the heat inducible cation channel dTRPA1 (Pooryasin, 2014) was expressed in random subsets of about 100 KCs using a Flp-out approach. Flies were trained aversively with electric foot shocks while dTRPA1 expressing KCs were activated by raising the ambient temperature above $25^{\circ} \mathrm{C}$. Subsequently, when tested on a temperature gradient, flies 
avoided the KCs reactivation by changing their temperature preference towards lower temperatures.

It has to be mentioned however, that some MB extrinsic neurons are required for the consolidation of olfactory memories in Drosophila. The dorsal paired medial (DPM) neurons that innervate the whole MBs, respond to olfactory stimulation and show increased responses and synaptic output after learning (Yu et al., 2005). They have been shown to release the memory relevant amnesiac gene product onto the MBs, and rescue of amnesiac in DPM neurons, in an amnesiac mutant background, is sufficient to restore appetitive and aversive olfactory associative memories (Keene et al., 2006; Waddell et al., 2000). Output from the DPM neurons is, however, only required during the memory consolidation phase, and it was shown that connectivity to the $a^{\prime} / \beta^{\prime} K C s$ is sufficient for proper memory consolidation (Keene et al., 2004, 2006).

Moreover, changes in odor responses were observed in the MB associated GABAergic anterior paired lateral (APL) neurons, and dopaminergic neurons after pairing odor and electric shock punishment (Liu and Davis, 2009; Liu et al., 2007; Riemensperger et al., 2005).

In conclusion, the collected evidences give overwhelming support for the localization of the olfactory memory trace in the KCs of the MBs. In conjunction with a MB extrinsic neuronal network, the memory in the KCs becomes consolidated. It will be interesting to find out, how communication and connectivity between KCs and memory-relevant MB output neurons are affected by the consolidation process.

\subsection{Reinforcement signaling}

The pathway of the neurons that perceive and convey the aversive or appetitive unconditioned stimuli in olfactory learning is until today not fully resolved. Nevertheless, it could be shown that dopaminergic or octopaminergic signaling is required during aversive or appetitive memory formation. Blocking output of TH-Gal4 positive neurons, in which Gal4 is expressed under control of the regulatory sequences of tyrosine hydroxylase, a key enzyme in the pathway for dopamine synthesis (FriggiGrelin et al., 2003), abolishes aversive memory formation (Schwaerzel et al., 2003). Appetitive learning is absent in flies mutated in the gene encoding tyramine betahydroxylase (Monastirioti et al., 1996), the enzyme catalyzing the last step in octopamine biosynthesis. Rescue of the gene by ectopic expression of tyramine betahydroxylase restores normal appetitive learning (Schwaerzel et al., 2003). Additionally, replacing the US by artificial activation of dopaminergic or octopaminergic 
neurons in larval Drosophila led to the formation of either an aversive or an appetitive memory (Schroll et al., 2006). In adult flies, the relevance of dopamine signaling for aversive memory formation could be confirmed in several studies by artificial activation of dopaminergic neurons as a replacement for an aversive electric shock (Aso et al.,

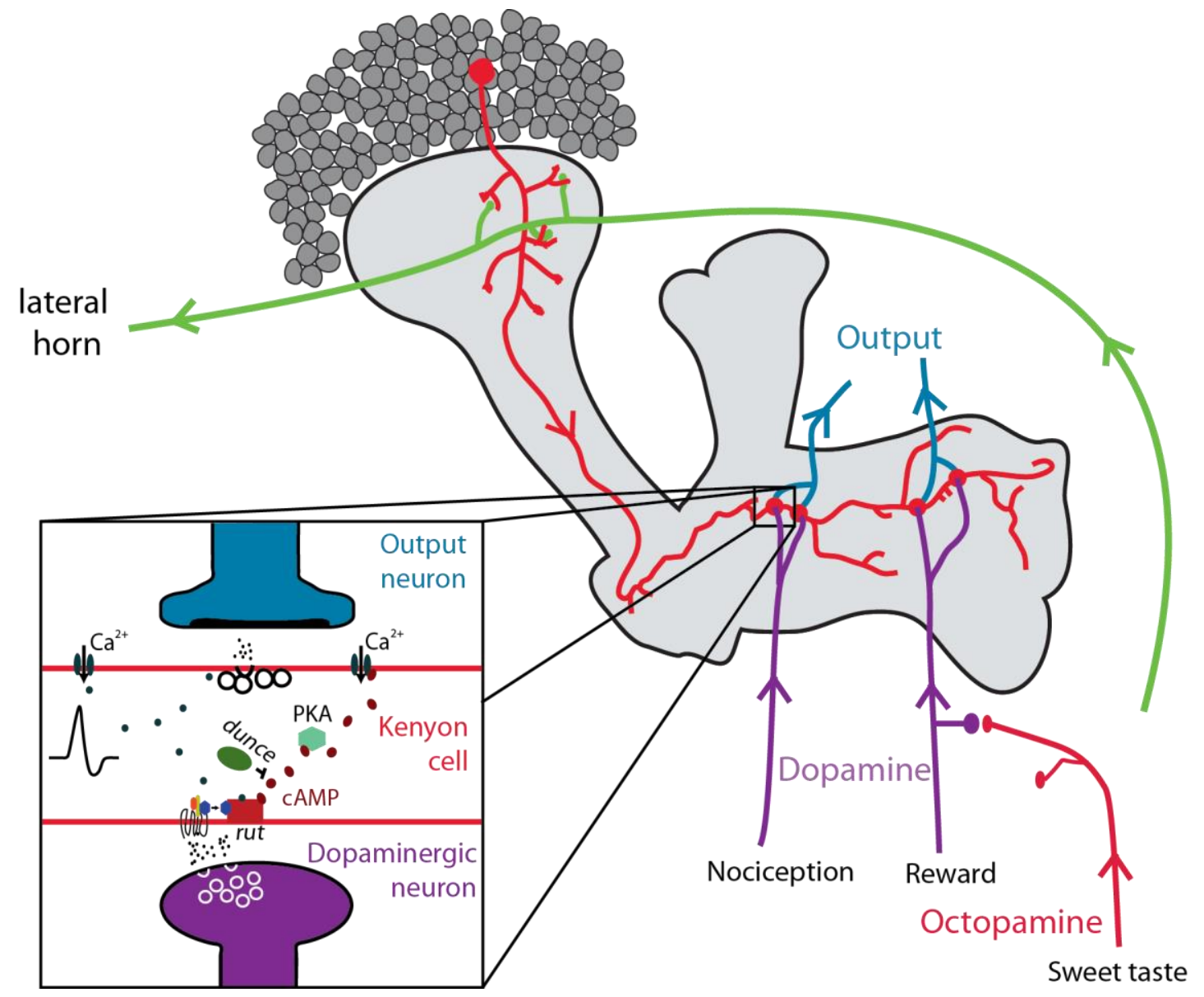

Figure 1.3. The model of olfactory associative learning in the Drosophila mushroom bodies.

Odors are conveyed by olfactory projection neurons (green) from the antennal lobes to the mushroom body and the lateral horn. Within the mushroom body calyx, projection neuron boutons connect to Kenyon cell (red) dendritic claws that carry the signal to the mushroom body lobes. Here, Kenyon cells form synaptic connections with dopaminergic (purple), and mushroom body output neurons (blue). Dopaminergic neurons were shown to convey reinforcement signals of nociception and reward to the mushroom bodies, while octopaminergic neurons, which are only required for certain types of appetitive memory formation, as induced by sweet taste, mediate their effect via dopaminergic neurons.

Inset: it is believed that the coincidence of odor-induced Kenyon cell activity and reinforcementinduced dopaminergic input is detected by a rutabaga-like adenylyl cyclase. This adenylyl cyclase produces CAMP upon simultaneous activation by calcium, deriving from depolarization, and Gprotein signaling, deriving from dopamine receptor binding. cAMP has various effects on the cell to regulate the communication to the mushroom body output neuron: for example via activation of protein kinase $A$, binding to cyclic nucleotide gated calcium-channels, or regulation of transcription by binding to CAMP response element-binding protein (CREB). The activity of CAMP can among others be modified by dunce-like phosphodiesterases that lead to the degradation of cAMP. Modified after Schwaerzel et al. (2002) and Waddell (2016) 
2010, 2012; Claridge-Chang et al., 2009). Furthermore, genetic rescue of the dopamine dDA1 receptor in the MBs, was sufficient to restore aversive memory formation (Kim et al., 2007; Qin et al., 2012). Although the nociceptive input by electric shock exposure is not clear, it could be shown that different aversive stimuli, which can induce an aversive memory, converge onto the same reinforcing dopaminergic neurons (Galili et al., 2014).

The role of octopamine in appetitive memory formation, however, had to be reclassified. At least in adult flies, appetitive memory inducing octopaminergic neurons were found to mediate their function via octopamine receptor expressing dopaminergic neurons (Burke et al., 2012). Indeed, it was shown that activation of a set of dopaminergic neurons was sufficient for the induction of an appetitive olfactory memory trace (Burke et al., 2012; Liu et al., 2012). Taken together, compartmentalized dopamine signaling has been recognized to be the main neuromodulation for reinforced memories, while its effect is dependent on the receiving $\mathrm{KCs}$ and the output network, in which they are embedded (Perisse et al., 2013a; Waddell, 2013).

In the model of olfactory associative learning, it is believed that coincidence detection of CS/US activity is achieved by an adenylyl cyclase (rutabaga-like) that via cAMP production activates signaling cascades leading to the modulation of KC output (figure 1.3). The adenylyl cyclase becomes activated by simultaneous stimulation by $\mathrm{Ca}^{2+} /$ calmodulin (CS pathway) and G-protein signaling (US pathway) (Fiala, 2007; Heisenberg, 2003). Indeed, disruption of G-protein signaling by ectopic expression of a dominant negative G-protein in KCs completely abolished memory formation (Connolly et al., 1996), and CAMP levels in the KCs were shown to be significantly increased after pairing KC activation with dopamine application (Tomchik and Davis, 2009). The necessity of CAMP-PKA signaling for neural plasticity is described in the previous chapter.

\subsection{Anatomy of the Drosophila mushroom bodies}

\subsubsection{Morphological organization of the Drosophila mushroom bodies}

Since the MBs are very prominent structures of the arthropod central nervous system their gross morphology was already well known in the $19^{\text {th }}$ century. At that time, though, the terminology as well as the language of publication was diverse and, for 

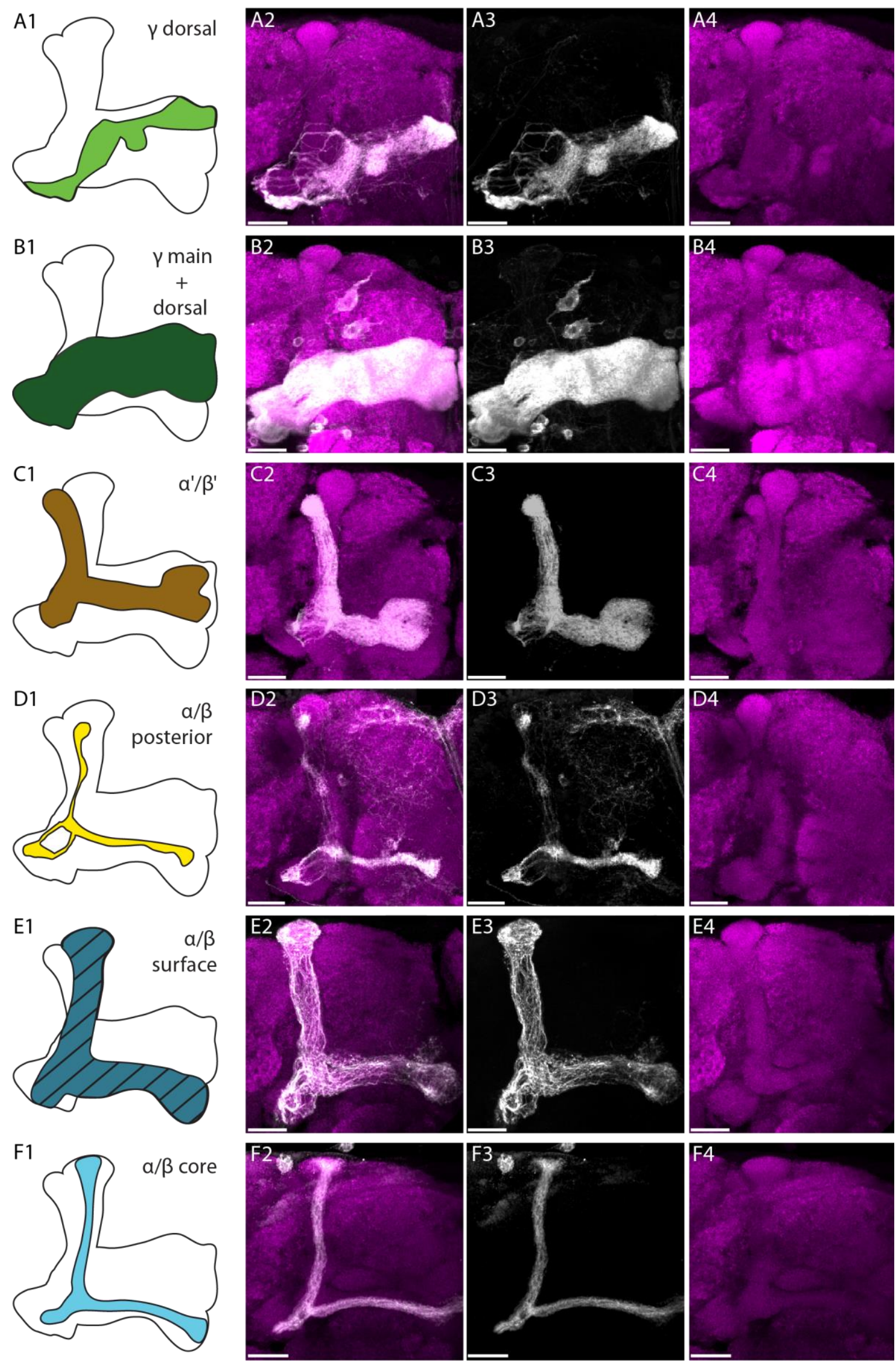

Figure 1.4. Types of Kenyon cells. Continued on next page. 
example, it was referred to them as "lobes à convolutions" in French, "Lappen mit Windungen", "Rind Körper", or "Pilzhutförmiger Körper" in German, while in English they were already known as the "mushroom bodies" (see Kenyon, 1896). The MBs can be subdivided into the calyx, where olfactory PNs synapse onto MB intrinsic Kenyon cells (Kenyon, 1896; Yasuyama et al., 2002), and which is surrounded by the cupshaped cell body layer, the peduncle, a stalk-like structure that extends through the mid-brain to connect the calyx to the lobes that lie in the anterior brain dorsal to the antennal lobes (figure 1.2). Across arthropod species the MBs are very remarkable structures due to their spatial orientation with the postero-dorsal localized calices and the anteriorly localized lobes, and the often observed division of the lobes into a medial part and a vertical part (Heisenberg, 1998; Strausfeld et al., 1998; Wolff and Strausfeld, 2015).

With the development of the Gal4/UAS system the analysis of MB anatomy in Drosophila was highly facilitated. First analyses of enhancer trap lines revealed a subdivision of the MB lobes into independent medially oriented $Y$ and $\beta$ lobes and vertically oriented $a$ and $a^{\prime}$ lobes (Yang et al., 1995). However, in an elegant study using antibody markers against MB enriched proteins it could be shown that the medial lobes consist of an additional $\beta^{\prime}$ lobe that is connected to the vertical $a^{\prime}$ lobe, and that the medial $\beta$ lobe is interconnected to the vertical a lobe. For the $\gamma$ lobe no vertical counterpart could be identified (Crittenden et al., 1998). Golgi stainings of individual $\mathrm{KCs}$ had indicated that the MBs consist of bifurcating cells that extend one branch into the vertical lobes and another into the medial lobes, and $\mathrm{KCs}$ that project only to the medial lobes (Mobbs, 1982; Yang et al., 1995). Thus, the MB lobes are comprised of bifurcating $a / \beta$ and $a^{\prime} / \beta^{\prime}$ KCs and non-bifurcating $y$ KCs (Crittenden et al., 1998).

Figure 1.4 continued.

Types of Kenyon cells that are differentiated by gene expression via different Gal4 driver lines, project to distinct areas within the mushroom body lobes. Axonal projections of Kenyon cells are illustrated by Gal4 driven expression of mcd8-GFP. Neuropil background is visualized by antibody labeling of presynaptic Nc82.

A) Expression pattern of $y$ dorsal cells driven by GMR93G04-Gal4, shown as illustration (A1), and as fluorescence images (A2-4).

B) Expression pattern of $\mathrm{Y}$ main and $\mathrm{Y}$ dorsal cells driven by 5HT1B-Gal4, shown as illustration (B1), and as fluorescence images (B2-4).

C) Expression pattern of $a^{\prime} / \beta^{\prime}$ cells driven by GMR35B12-Gal4, shown as illustration (C1), and as fluorescence images (C2-4).

D) Expression pattern of $a / \beta$ posterior cells driven by GMR71F03-Gal4, shown as illustration (D1), and as fluorescence images (D2-4).

E) Expression pattern of $a / \beta$ surface cells driven by GMR14G01-Gal4, shown as illustration (E1), and as fluorescence images (E2-4).

F) Expression pattern of $a / \beta$ core cells driven by GMR65A01-Gal4, shown as illustration (F1), and as fluorescence images (F2-4). 
Further studies, analyzing a vast range of enhancer-trap and promoter Gal4 expression patterns, could show that each of the mentioned KC types could be subdivided into at least two additional subtypes which are not only distinguished based on gene expression patterns but also morphologically (Aso et al., 2009, 2014a; Butcher et al., 2012; Strausfeld et al., 2003; Tanaka et al., 2008; figure 1.4). Those subtypes are the $\mathrm{Y}$ main ( $\sim 600$ cells) and $\mathrm{Y}$ dorsal ( $\sim 5$ cells) KCs, the $a^{\prime} / \beta^{\prime}$ middle ( $\sim 140$ cells) and $\mathrm{a}^{\prime} / \beta^{\prime}$ anterior-posterior ( 210 cells) $\mathrm{KCs}$, and the $\mathrm{a} / \beta$ posterior ( $\sim 90$ cells), $\mathrm{a} / \beta$ surface ( $\sim 500$ cells), and $a / \beta$ core ( $\sim 400$ cells) KCs. The $a / \beta$ posterior and the $Y$ dorsal KCs are special in the sense that their dendrites arborize outside the main calyx. Two accessory calices lie dorsal and ventral of the main calyx. The dorsal accessory calyx is exclusively innervated by $a / \beta$ posterior $\mathrm{KCs}$ and the ventral accessory calyx is exclusively innervated by $\mathrm{Y}$ dorsal $\mathrm{KCs}$ (the term dorsal used in $\mathrm{Y}$ dorsal $\mathrm{KCs}$ indeed refers to their relative position within the lobes not to their calycal arborizations) (Aso et al., 2009, 2014a; Butcher et al., 2012; Tanaka et al., 2008).

\subsubsection{Organization of mushroom body extrinsic neurons}

The MBs are innervated by a large number of extrinsic neurons that contribute to approximately one quarter of the overall MB structure and morphology (Aso et al., 2014a). Two sets of MB extrinsic neurons are outstanding because they are each represented by only one cell per hemisphere but are still strongly interconnected with the MB neuropil. The anterior paired lateral ( $A P L)$ neurons were shown to be GABAergic (Liu and Davis, 2009) and can be seen in fluorescence microscopy to innervate all MB substructures including the peduncle and the accessory calyx (Tanaka et al., 2008). The dorsal paired medial (DPM) neurons do not innervate the MB calyx and neither most of the peduncle. Their processes are found in the inferior part of the peduncle, and in all of the MB lobes (Ito et al., 1998; Tanaka et al., 2008). They have been shown to release a neuropeptide that is the product of the amnesiac gene (Waddell et al., 2000), serotonin (Lee et al., 2011), and potentially GABA (Haynes et al., 2015). The majority of $M B$ associated extrinsic neurons are aminergic neurons that often appear in the brain as clusters of cell bodies. A recent study made use of the GFP reconstitution across synaptic partners (GRASP) technique (Feinberg et al., 2008) to detect and quantify contacts between different aminergic Gal4 lines and KCs. Signals of reconstituted GFP were detected throughout the MBs, though the most abundant contacts were made by dopaminergic neurons, whereas octopaminergic neurons 
sparsely innervated the MBs. Also a variety of non-DPM serotoninergic neurons were found to form contacts to KCs in subregions of the lobes (Pech et al., 2013b).

The innervation patterns of dopaminergic neurons have been subject to extensive research because of their role in associative memory formation (Liu et al., 2012; Schwaerzel et al., 2003) and the specificity of their arborizations (Aso et al., 2014a; Ito et al., 1998; Tanaka et al., 2008). The MB lobes can be subdivided into 15 compartments based on the innervations of dopaminergic and MB output neurons (figure 1.5). Each of the three main $M B$ lobes $\left(\gamma, a^{\prime} / \beta^{\prime}, a / \beta\right)$ consists of five compartments. From central to distal the compartments have been named with increasing numbers: $\gamma 1-5, a^{\prime} 1-3, \beta^{\prime} 1-2, a 1-3$, and $\beta 1-2$. All of the 15 compartments are innervated by axonal terminals of dopaminergic neurons that are distinguished in 20 different types, from which 17 exclusively arborize within a single compartment. Out of this set of dopaminergic neurons one type is special in the way that it has

A

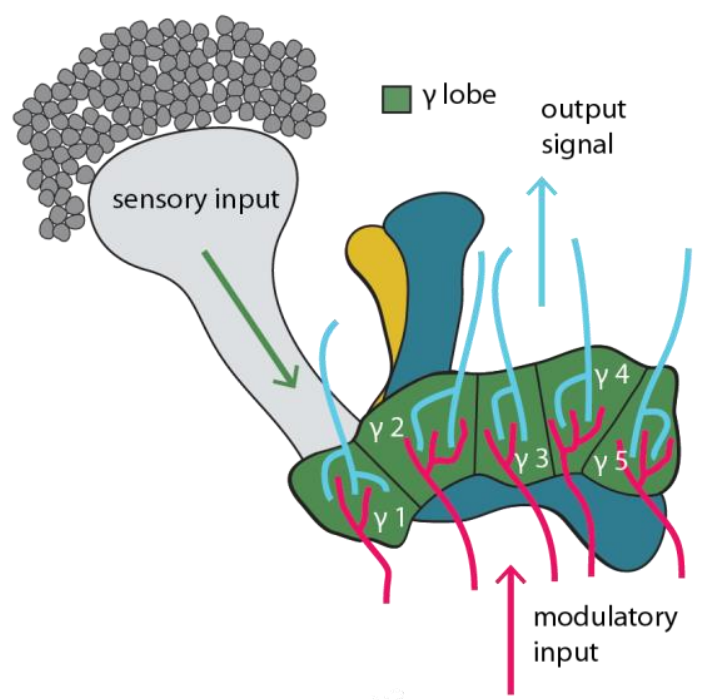

B

$\square a^{\prime} / \beta^{\prime}$ lobe

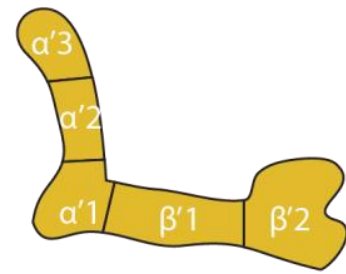

C

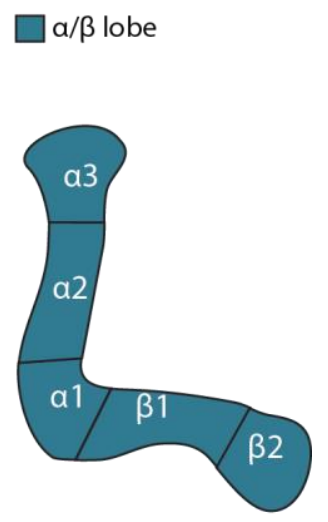

Figure 1.5. Compartmentalized organization of the mushroom body lobes.

The mushroom body lobes can be subdivided into 15 compartments based on the innervation patterns of extrinsic dopaminergic and mushroom body output neurons. A total of 20 dopaminergic neuron types and 21 mushroom body output neuron types innervate primarily one single compartment. Thus, each compartment is defined by the axonal terminals of at least one dopaminergic neuron type, and the dendritic innervations of at least one mushroom body output neuron type.

A) The organization of extrinsic innervations to the mushroom body lobes is illustrated with the $y$ lobe. Kenyon cells that comprise the $y$ lobe receive sensory input in the calyx, and project through the peduncle into the lobes, where they are densely innervated by modulatory dopaminergic neurons and mushroom body output neurons that likely link the mushroom body to motor output areas. The arbors of the two cell types are restricted to compartments $y 1-5$.

B) Illustration of the $a^{\prime} 1-3$ compartments and the $\beta^{\prime} 1-2$ compartments.

C) Illustration of the a1-3 compartments and the $\beta 1-2$ compartments.

Modified after Cohn et al. (2015). 
dendritic processes within the $\mathrm{y} 1$ and $\mathrm{y} 2$ compartments and axon termini in the $\mathrm{y} 4$ compartment. In total, there are 100 dopaminergic neurons that have their cell bodies in the PAM cluster, and which innervate compartments $\gamma 3, \gamma 4, \gamma 5, \beta^{\prime} 1, \beta^{\prime} 2, a 1$, $\beta 1$, and $\beta 2,6-7$, dopaminergic neurons of the PPL1 cluster that innervate compartments $y_{1}, y_{2}, a^{\prime} 1, a^{\prime} 2, a^{\prime} 3, a 2$, and $a 3$, and around 6 cells of the PPL2ab cluster, which extend their neurites into the calyx (Aso et al., 2014a; Mao and Davis, 2009; Riemensperger et al., 2005; Tanaka et al., 2008).

MB output neurons were found to innervate exactly the same lobular compartments as the dopaminergic neurons. They consists of 21 different cell types. Except for the MB output neuron type that extends dendritic arbors to the $\beta^{\prime} 1$ and consists of up to nine cells, other MB output neuron types consists of one to two cells. Interestingly, there is one MB output neuron with axon termini in the $\mathrm{Y} 1$ and $\mathrm{y} 2$ compartment and dendrites in the $\mathrm{y} 4$ compartment. Thus, considering the dopaminergic neuron described above with complementary projections, there seems to be a microcircuit formed between y compartments 1, 2, and 4 .

In terms of neurotransmitter expression MB output neurons fall into three classes: glutamatergic, GABAergic, and cholinergic. The dendritic arborizations of MB output neurons lie in the different MB compartments, where they synapse onto MB intrinsic $\mathrm{KCs}$ in close proximity to the respective dopaminergic neuron type. Interestingly, axonal termini of MB output neurons and dendritic processes of dopaminergic neurons partly arborize within the same brain regions, i.e. the crepine (located around the medial lobes), the superior medial protocerebrum, the superior intermediate protocerebrum, and the superior lateral protocerebrum, suggesting the presence of a recurrent network between the two cell types (Aso et al., 2014a).

In conclusion, the anatomically identified network between KCs, MB output neurons, and dopaminergic neurons represents a memory circuit assisted by the APL and DPM neuron that is predetermined to process multimodal sensory input in spatially defined computational units, the 15 compartments of the $\gamma, a^{\prime} / \beta^{\prime}$, and $a / \beta$ lobes (Aso et al., 2014a). Thus, modifications of the connectivity between KCs and MB output neurons that underlie behavioral adaptation are likely to occur in a compartment specific manner, dependent mainly on modulatory dopaminergic input.

\subsection{Development of the mushroom bodies}

Clonal analysis of KCs using Bromodeoxyuridine labeling of proliferating neuroblasts revealed that the MBs are generated out of four neuroblasts (Ito and Hotta, 1992), 
which were shown by genetic mosaic analysis to contribute equally to all known KC types of the MBs (Ito et al., 1997). Each of the MB neuroblasts sequentially produces Y main KCs during L1 and L2 larval stage, $a^{\prime} / \beta^{\prime} K C$ s during $L 3$ larval stage, and $a / \beta$ KCs during pupal stage (Kurusu et al., 2002; Lee et al., 1999; Zhu et al., 2003), while $Y$ dorsal KCs are already generated in the embryo (de Belle and Heisenberg, 1994; Butcher et al., 2012; Kunz et al., 2012; Yu et al., 2013). The subdivisions of the $a / \beta$ lobes, namely the $a / \beta$ posterior, the $a / \beta$ surface, and the $a / \beta$ core neurons are

A
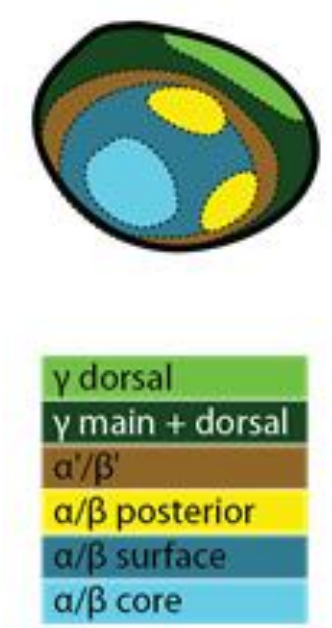
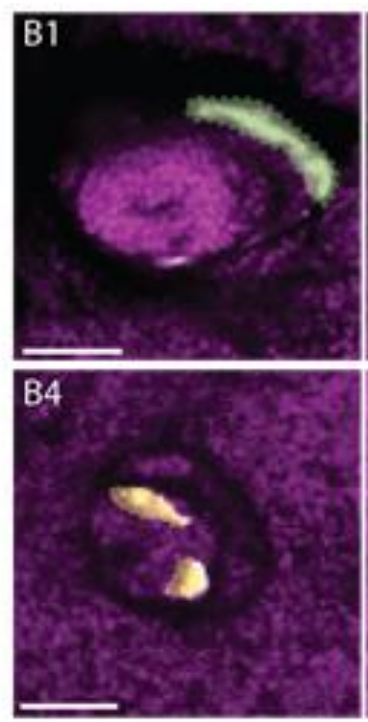
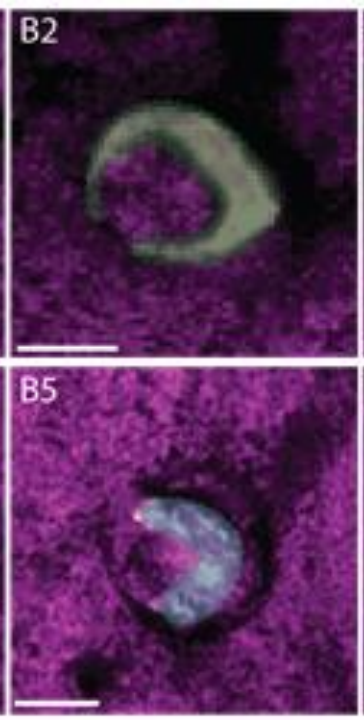
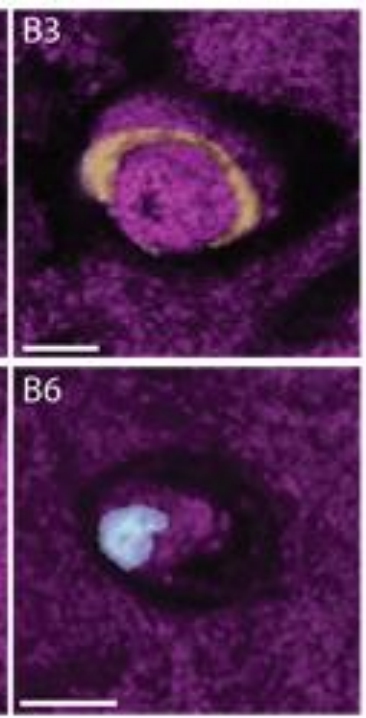

Figure 1.6. Projections of Kenyon cell types in the peduncle.

Different types of Kenyon cells occupy distinct areas within the mushroom body peduncle. Kenyon cells are differentiated by birth order and later born cells extend their axon into the middle of the peduncle, thereby pushing earlier born cells towards the periphery.

A) Illustration of the areas in the peduncle that are innervated by projections of distinct types of Kenyon cells.

$B$ ) Confocal images of Gal4 driven mcd8-GFP expression in $y$ dorsal cells (B1) y main and $y$ dorsal cells (B2), $a^{\prime} / \beta^{\prime}$ cells (B3), $a / \beta$ posterior cells (B4), $a / \beta$ surface cells (B5), and $a / \beta$ core cells (B6). Neuropil background is visualized by Nc82 antibody labeling. Scale bars $=10 \mu \mathrm{m}$.

generated sequentially during pupal stage in the order as mentioned (Zhu et al., 2003). Newly born KCs extend their projections through the core of the peduncle, thereby pushing the already generated $\mathrm{KC}$ neurites towards the periphery so that different $\mathrm{KC}$ types are arranged in the peduncle as concentric circles (Kurusu et al., 2002; figure 1.6). Transitions from the generation of one cell type to the next happen abruptly (Kunz et al., 2012; Lee et al., 1999). However, they depend on external cues and the developmental stage of the animal. Blockage of transition between developmental stages, and thereby extending the time of the animals' development, by starvation of larvae, led to an increased number of the KC type generated at the respective time (Lin et al., 2013). Cells generated by different neuroblasts are separated in their dendritic arborizations within the calyx, in the way that they occupy 
separated vertical columns with minimal overlap (figure 1.1). The $a / \beta$ posterior cells are excepted because they were found to intermingle within the area of the dorsal accessory calyx (Aso et al., 2014a; Ito et al., 1997; Lin et al., 2007). MB neuroblasts become inactive from the $9^{\text {th }}$ day after larval hatching or approximately 4 days after pupae formation. Neurogenesis of KCs in adult Drosophila does not happen (Ito and Hotta, 1992; Lee et al., 1999).

Interestingly, KCs of the $\mathrm{Y}$ type undergo restructuring during pupal stage. Their dendritic and axonal processes are pruned and degraded and regrow again into the MB medial lobes only (in contrast, $Y$ neurons grown during larval life project into the vertical and medial lobes) (Technau and Heisenberg, 1982). This especial remodeling has indeed become a research field on its own for the study of pruning processes in dendrites and axons (Yu and Schuldiner, 2014).

In sum, KC subtypes are distinguished by their birth order, while $\mathrm{Y}$ neurons are special because they undergo remodeling during pupal stage. Thus, identity of KCs is so far determined as an interplay of genetic, morphological, and developmental characteristics.

\subsection{Functional anatomy of mushroom body subdivisions}

\subsubsection{Functional anatomy of Kenyon cell classes}

Classically, the functional subdivision of the MBs was simple. $Y$ neurons are required for short-term memory formation (Blum et al., 2009; Zars, 2000), the $a^{\prime} / \beta^{\prime}$ neurons in conjunction with the DPM neurons are responsible for memory consolidation (Cervantes-Sandoval and Davis, 2012; Keene et al., 2006; Krashes et al., 2007; Waddell et al., 2000), and the $a / \beta$ neurons are needed for memory retrieval (Dubnau et al., 2001; Krashes et al., 2007; McGuire, 2001). Strikingly, conditioning flies of the alpha-lobes-absent mutant, which lack either the vertical a/ $a^{\prime}$ lobes or the medial $\beta / \beta^{\prime}$ lobes revealed that none of the four lobes is required for short-term memory formation, leaving the $y$ lobes as the most likely mediator. Additionally, it was found that flies missing the vertical $a / a^{\prime}$ lobes were defective in long-term memory formation (Pascual and Preat, 2001). In more detailed analyses of the requirements of proper rutabaga functioning for memory, Blum et al (2009) and Scheunemann et al (2012) showed that the $a / \beta$ and $y$ lobes have synergistic effects on the formation of short-, and long-term memories, and that cAMP signaling in $\alpha^{\prime} / \beta^{\prime}$ neurons was dispensable. 
Cervantes-Sandoval et al (2013) examined the precise need of output from different MB lobes in a very comprehensive study of short- and long-term aversive and appetitive memories making use of the temperature sensitive shibire mutant of dynamin (Kitamoto, 2001). In sum, output from all lobes is required for aversive and appetitive short-term memory, especially the prime lobes for appetitive short-term memory, and the $a / \beta$ lobes for the retrieval of aversive and appetitive long-term memory (Cervantes-Sandoval et al., 2013). Complete blockage of long-term memory retrieval, however, is only achieved by inhibiting transmission from both $a / \beta$ and $y \mathrm{KCs}$ (Xie et al., 2013). Functional calcium imaging revealed higher relative activity of $a^{\prime} / \beta^{\prime}$ neurons in early memory and increased CS+ responses in $a / \beta$ neurons in late memory for both appetitive and aversive memories (Cervantes-Sandoval et al., 2013). Looking at the subsets of $a / \beta$ neurons, it was found out that $a / \beta$ surface and core neurons are required for retrieval of $3 \mathrm{hr}$ appetitive memory but only $a / \beta$ surface neurons for retrieval of $3 \mathrm{hr}$ aversive memory (Perisse et al., 2013b).

There are two subsets of KCs that are special in the way that they build dendritic processes outside the main calyx: $\gamma$ dorsal and $a / \beta$ posterior $K C s$ that also have $a$ stereotyped projection pattern in the MB lobes. The KCs of the $Y$ dorsal subset have their dendritic arborizations in the ventral accessory calyx (Butcher et al., 2012), and project to defined areas in the lobes, mainly in $\mathrm{y} 2, \mathrm{\gamma} 3$, and to a dorsal tip in $\mathrm{y} 5$ (see figure 1.4). A recent study has shown that visual PNs from the optic lobes provide input to the $\mathrm{Y}$ dorsal KCs, and that indeed, $\mathrm{Y}$ dorsal KCs are necessary for visual associative learning but not for olfactory learning (Vogt et al., 2016). Additionally, $Y$ dorsal KCs have been implicated in sleep homeostasis (Sitaraman et al., 2015).

$a / \beta$ posterior neurons were found to extend their dendritic neurites into the dorsal accessory calyx and project their axonal fibers to unique areas in the lobes, thereby forming several peculiar nodes (Tanaka et al., 2008; and figure 1.4). Their role in behavior has so far not been resolved. It has been reported that they are involved in aversive olfactory long-term memory retrieval (Chen et al., 2012), however, this hypothesis was not supported in another study that even showed that they are inhibited by olfactory input (Perisse et al., 2013b). Furthermore, no anatomical connections were found between olfactory PNs and $a / \beta$ posterior KCs (Tanaka et al., 2008).

KCs have been analyzed for the kind of neurotransmitter they produce and release. For a long time this question could not be answered in a satisfactory manner. Glutamate, taurine, acetylcholine, and aspartate have been suggested as transmitters present in subsets of KCs (Sinakevitch et al., 2001; Strausfeld et al., 2003). However, 
it was not until recently that acetylcholine could be proven by RNA interference (Fire et al., 1998), pharmacology, and physiology to be the major transmitter released from KCs onto MB output neurons (Barnstedt et al., 2016). Short neuropeptide F (sNPF), the insect homolog of mammalian neuropeptide $Y$ (Brown et al., 1999), is expressed in all but $a^{\prime} / \beta^{\prime}$ KCs as a co-transmitter (Crocker et al., 2016; Johard et al., 2008; Lee et al., 2004). It could be shown that SNPF, when released onto olfactory receptor neurons, is involved in regulating food responses of starved flies (Root et al., 2011). Moreover, sNPF release by KCs is necessary for appetitive olfactory memory formation, establishing a role of SNPF in the MBs as a functional neuromodulator for appetitive behavior (Knapek et al., 2013).

\subsubsection{Functional anatomy of mushroom body compartments}

The most interesting feature of the MBs is the described anatomical subdivision into compartments based on the innervation patterns of dopaminergic and MB output neurons (Aso et al., 2014a). This structural organization implicates a corresponding functional organization, i.e. the processing of different forms of memory in independent computational units. The synapses between KCs and MB output neurons would be modified in a compartment specific way. Indeed, it could be shown that input to the $\gamma 1$ compartment is accounted for aversive short-term memory, while parallel traces of aversive memory could be formed in $a^{\prime} 1, a 2, a^{\prime} 2$, and $\beta 2$ with different stabilities (Aso et al., 2012). Surprisingly, Perisse et al (2013b) reported that activation of dopaminergic neurons projecting to $\beta 1$, and $\beta 2$ was sufficient to induce an appetitive memory trace. A possible solution is that the $\beta 1$ appetitive memory is overcompensated by the aversive $\beta 2$ memory. A detailed analysis of appetitive memories by Yamagata et al (2015) based on the findings of Liu et al (2012), who found PAM cluster dopaminergic neurons to be responsible for appetitive memory formation, revealed PAM-a1 dopaminergic neurons to be accountable for long-term appetitive memory and $\gamma_{5}, \gamma^{4}$, and $\beta^{\prime} 2$ innervations to be accountable for appetitive short-term memory. Similarly, induced activity in $\gamma^{4}$ and $\mathrm{\gamma} 3$ was sufficient for the formation of an appetitive memory trace (Aso et al., 2012). In an interesting case about competing memories with different qualities it was shown that training with DEET supplied sugar reward induced two opposing memories in parallel. One aversive short-term memory due to the aversive effects of DEET and another middle-/longterm appetitive memory due to the sugar reward (Das et al., 2014). A comprehensive 
overview of the abilities of different dopaminergic neurons for the induction of different memories in different compartments, analyzed for their training requirements for memory formation, memory capacities, extinction dynamics, and flexibility to form new associations is given in Aso and Rubin (2016). In their assay they used identified rewarding dopaminergic neurons that project to $a 1, \beta 1, \beta 2, \beta^{\prime} 2$, and $\gamma^{5}$ and punitive dopaminergic neurons projecting to $\gamma_{1}, \gamma_{2}, a^{\prime} 1, a 2, a^{\prime} 2$, and $a 3$ (Aso and Rubin, 2016). Investigation of dopaminergic output to $\mathrm{KCs}$ of the $\mathrm{Y}$ lobe revealed a functional subdivision into reward activated $\mathrm{\gamma} 4$, and 5 , and punishment activated $\mathrm{\gamma} 2$, and 3 compartments. The responses of KCs were specific to the respective compartmental input as shown by volumetric $\mathrm{Ca}^{2+}$ imaging. Interestingly, the internal state of the fly, i.e. being calm or active (flailing) under the microscope, was represented as dopaminergic activity in the same compartmental order (Cohn et al., 2015). In concordance to that, input from $\mathrm{y} 4$ dopaminergic neurons is necessary in water deprived flies for appetitive memory formation (Lin et al., 2014b), and inputs from $y 5$ dopaminergic neurons are necessary for signaling of sucrose reward in food deprived flies (Huetteroth et al., 2015). Thus, dopaminergic neurons not only signal the valence of external conditions but also the relevance of internal states, like thirst or hunger, in order to gate associative learning.

In the model of olfactory associative learning it is the synapses between KCs as the mediators of the odor stimulus on the one hand and MB output neurons as the relay system to motor output areas on the other hand that are prone to reinforcement induced changes (Fiala, 2007; Heisenberg, 2003). A comprehensive analysis carried out by (Aso et al., 2014b) included all 21 so far identified output neurons. The activation of some neurons was avoided by the flies, whereas the activation of other neurons was approached, or had no effect at all. Significant aversive short-term memory defect was found when output from the $\mathrm{y} 1$ output neuron was blocked, and an aversive long-term memory defect when output from a2, a'1, and a'3 output neurons was blocked during memory retrieval. a1, a3, $\beta^{\prime} 2, a^{\prime} 1, a^{\prime} 2, y 2$, and $y^{5}$ output neurons were shown to be involved in appetitive short- and $\beta^{\prime} 2, a^{\prime} 1, a^{\prime} 2, a^{\prime} 3, \gamma^{2}, \gamma^{4}$, and $\mathrm{y} 5$ output neurons in appetitive long-term memory. The output neurons necessary for visual memory retrieval were mostly the same as for olfactory memory retrieval, while others showed specialized involvement in visual memory, e.g. one output neuron that arborizes only in the a 2 posterior KCs. Interestingly, activity of output neurons that was aversive for the flies was also wake promoting, and activity of output neurons that was attractive for the flies was also sleep promoting indicating that MB output 
might express a general motivational state, e.g. feeling comfortable or stressed (Aso et al., 2014b).

Several output neurons have been analyzed in some detail. It was found that output from a2 and a'3 was required for aversive short- and long-term memory retrieval, and that the learning induced odor response of the output neuron was reduced (Séjourné et al., 2011). This finding implicates that the corresponding dopamine signal of punishment (Aso et al., 2012) in a2 paired with an odor stimulus leads to a weakening of the synapses between the KCs and the output neuron; an observation also supported for appetitive learning by imaging of the $\mathrm{Y} 4$ output neuron (Cohn et al., 2015), and in another study measuring aversive learning responses in $\mathrm{Y} 1$ (Hige et al., 2015b). In all cases, the odor evoked response in the respective output neuron was lower after learning. In contrast, the output neuron described by Pai et al (2013) that has dendritic arborizations in a3, showed increased activity after aversive training in response to stimulation with the CS+ (Pai et al., 2013), but decreased odor responses to the CS+ after appetitive memory formation (Plaçais et al., 2013). Likewise, in a group of glutamatergic output neurons residing in $\gamma^{2}, \beta^{\prime} 2$, and $\beta 2$ appetitive conditioning evokes a depression, while aversive conditioning leads to an increase of the output neurons responses of the same compartments (Owald et al., 2015). Therefore, bi-directional modification of KC-output neuron synapses dependent on the type of learning seems to be a principle used for valence specific information storage at least in some of the compartments.

In a normal situation, the activity of cells having a negative valence is balanced out against the activity of cells having a positive valence. Learning can mediate approach or avoidance via compartment specific modulation of KC-MB output neuron synapses to skew the overall MB output neuron population response (for review see Owald and Waddell, 2015) The (counter-) balancing of MB output neurons is highly individualized. Measuring odor evoked responses of the same output neuron in different individuals demonstrated a surprising variability, which, however, is not found between hemispheres in the same fly. The variability between flies seems to be experience dependent, since it was lost in rutabaga mutants that are defective in the cAMP pathway (Hige et al., 2015a).

Although changes in $\mathrm{KC}$ responses after learning have been observed in various experiments, a direct evidence for neural plasticity is still missing. In a series of elegant experiments, taking the $\mathrm{\gamma} 1$ compartment as a model system, Hige et al (2015b) demonstrated that first, odor-dopamine pairing induced a reduction in the corresponding MB output neurons firing rate, second, the excitability of single KCs or 
the KC population response was not changed after pairing, and third, that odordopamine pairing induced severe depression of excitatory postsynaptic currents, which is independent of postsynaptic spiking in contrast to findings in the locusts' MBs (Cassenaer and Laurent, 2007, 2012). Interestingly, measurements in another compartment, i.e. a2, using the same training regime revealed no postsynaptic changes. Only KC-dopamine pairing for a longer period induced postsynaptic depression in the a2 MB output neuron, fitting to the role of a2 in long-term memory retrieval (Séjourné et al., 2011). In sum, neural plasticity at the KC-MB output neuron synapse seems to be localized to the postsynaptic cell, however, local changes at the KC presynapses cannot be excluded.

Furthermore, MB volume changes were observed as a result of housing conditions of adult flies (Heisenberg et al., 1995). In the honey bee, Apis mellifera, the MB volumes were shown to increase with experience when they undergo transition from workers to foragers (Durst et al., 1994; Withers et al., 1993). Volume changes are strictly experience-dependent and are accompanied by the outgrowth of dendritic arbors (Farris et al., 2001). Structural plasticity of individual Drosophila KCs remains to be demonstrated.

\subsection{Scope of the thesis}

The requirement of KCs in various, mainly learning related, behaviors has been described in detail. Much emphasis was laid on the underlying molecular machinery (reviewed for example in Davis, 1996, 2004; Margulies et al., 2005; Roman and Davis, 2001; Waddell and Quinn, 2001) and the investigation of identified KC subclasses and $\mathrm{KC}$ compartments in the context of their functional contribution to the MB circuitry (reviewed for example in Davis, 2011; Fiala, 2007; Heisenberg, 2003; Owald and Waddell, 2015; Pitman et al., 2009). The MB morphology has been described in detail with an emphasis on network components (Aso et al., 2014a; Tanaka et al., 2008). However, the description of single KCs, the supposed carriers of memory, has been neglected so far, except for studies of development and disease (Furukubo-Tokunaga et al., 2016; Lee and Luo, 2001; Lee et al., 2000; Tessier and Broadie, 2008; Yu and Schuldiner, 2014). The investigation of single KC functional anatomy has the chance to complement our understanding of how neuronal systems manage to form and retrieve memories. Here, I aim to lay the foundation for this work by taking the $y$ lobe as a model system and to anatomically describe the contributing KCs in a quantitative comprehensive manner. To this end, a set of 75 single $\mathrm{Y} \mathrm{KC}$ images was collected using 
mosaic analysis with a repressible cell marker (Lee and Luo, 1999). KC morphologies were digitalized in order to quantify their structure. This was done in respect to MB subregions, i.e. the calyces, and the lobes with their compartments that were identified by background marker expression. The quantified parameters were used to compare and classify single KCs using standard statistical procedures as well as cluster analysis and principle component analysis.

Finally, it was investigated how exactly KCs might contribute to the formation of memories in terms of structural transformations. Thus, using a similar approach as described above, the flexibility of KCs, deriving from all major MB subclasses, to adapt structurally to different environmental conditions was tested. To this end, Drosophila imagos were exposed to different feeding regimes, which were shown to affect KCdopaminergic neuron connectivity and KC-MB output neuron connectivity ( $T$. Riemensperger, personal communication).

The acquired information is discussed in the context of the MB model system for olfactory associative learning and memory. Understanding mnemonic processes at the single cell level is the next step on the way to a functional understanding of an insect central nervous system. 


\section{Materials and Methods}

\subsection{Materials}

\subsubsection{Fly strains}

The following fly strains were used in this thesis. New fly strains were generated by crossbreeding as described in section 2.2.1 based on the strains found in this list.

\begin{tabular}{|c|c|c|}
\hline Genotype & Donor & Reference \\
\hline $\mathrm{w}^{-} ; ;$GMR35B12-Gal4 & $\begin{array}{l}\text { Bloomington } \\
(\# \text { 49822) }\end{array}$ & Jenett et al., 2012 \\
\hline $\mathrm{w}^{\prime} ; ;$ GMR14G01-Gal4 & $\begin{array}{l}\text { Bloomington } \\
(\# \text { 48655) }\end{array}$ & Jenett et al., 2012 \\
\hline $\mathrm{w}^{-} ; ;$GMR65A01-Gal4 & $\begin{array}{l}\text { Bloomington } \\
(\# \text { 39328) }\end{array}$ & Jenett et al., 2012 \\
\hline $\mathrm{w}^{-} ; ;$GMR71F03-Gal4 & $\begin{array}{l}\text { Bloomington } \\
(\# \text { 39595) }\end{array}$ & Jenett et al., 2012 \\
\hline $\mathrm{w}^{-} ; ;$GMR93G04-Gal4 & $\begin{array}{l}\text { Bloomington } \\
(\# \text { 40661) }\end{array}$ & Jenett et al., 2012 \\
\hline $\mathrm{Y}, \mathrm{w}^{-}, \mathrm{FRT} 19 \mathrm{~A} ; ;$ & $\begin{array}{l}\text { Bloomington } \\
(\# \text { 1744) }\end{array}$ & Xu and Rubin, 1993 \\
\hline hsFlp, tubP-Gal80, w', FRT19A;; & $\begin{array}{l}\text { Bloomington } \\
(\# \text { 5132) }\end{array}$ & $\begin{array}{l}\text { Golic, 1991; Lee and } \\
\text { Luo, 1999; Xu and } \\
\text { Rubin, } 1993\end{array}$ \\
\hline $\mathrm{w}^{-} ; ;$10xUAS:mcd8::GFP & $\begin{array}{l}\text { Bloomington } \\
(\# \text { 32184) }\end{array}$ & Pfeiffer et al., 2010 \\
\hline w; $^{-}$MB247-DsRed; MB247-DsRed & André Fiala & $\begin{array}{l}\text { Riemensperger et al., } \\
2005\end{array}$ \\
\hline
\end{tabular}




\begin{tabular}{|c|c|c|}
\hline y, w'; UAS>stop>mcd8-GFP/CyO; & $\begin{array}{l}\text { Bloomington } \\
(\# 30125)\end{array}$ & Yu et al., 2010 \\
\hline 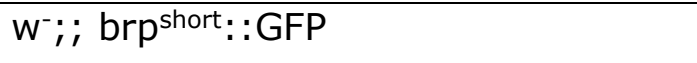 & Stephan Sigrist & Fouquet et al., 2009 \\
\hline $\begin{array}{l}\text { y, w; } 20 X U A S-6 X m C h e r r y-H A ~ / C y O, \\
\text { Wee-P; Dr/TM6C, Sb, Tb }\end{array}$ & $\begin{array}{l}\text { Bloomington } \\
\text { (\# 52267) }\end{array}$ & Shearin et al., 2014 \\
\hline $\begin{array}{l}\text { y, w; Sp/CyO, Wee-P; 20XUAS- } \\
\text { 6XmCherry }\end{array}$ & $\begin{array}{l}\text { Bloomington } \\
(\# \text { 52268) }\end{array}$ & Shearin et al., 2014 \\
\hline $\mathrm{w}^{-} ; ; ;$OK107-Gal4 & Robert Kittel & Connolly et al., 1996 \\
\hline $\mathrm{w}^{-} ; ; ;$OK107-Gal4/ci & $\begin{array}{l}\text { Bloomington } \\
(\# 854)\end{array}$ & $\begin{array}{l}\text { Connolly et al., 1996; } \\
\text { Von Ohlen and Hooper, } \\
1999\end{array}$ \\
\hline $\mathrm{w}^{-} ; ;$MB247-Gal4 & André Fiala & Schulz et al., 1996 \\
\hline $\mathrm{w}^{-} ; ;$5HT1B-Gal4 & $\begin{array}{l}\text { Bloomington } \\
(\# 27637)\end{array}$ & Yuan et al., 2005 \\
\hline w; MB247-GCaMP 3.0 (4M); & André Fiala & Pech et al., $2013 \mathrm{~b}$ \\
\hline
\end{tabular}

\subsubsection{Fly food}

\subsubsection{Standard cornmeal food}

The following ingredients were used to make $10 \mathrm{~L}$ of standard cornmeal food:

\begin{tabular}{|l|l|l|}
\hline Name and quantity & Company & Address \\
\hline $102.5 \mathrm{~g}$ agar & Gourvita GmbH & $\begin{array}{l}\text { Adam-Opel-Str. 19 } \\
\text { DEU-63322 Rödermark }\end{array}$ \\
\hline $100 \mathrm{~g}$ soy flour & $\begin{array}{l}\text { Pflanzensaftwerk } \\
\text { GmbH \& Co. KG }\end{array}$ & $\begin{array}{l}\text { Hutwiesenstraße 14 } \\
\text { DEU-71106 Magstadt }\end{array}$ \\
\hline $180 \mathrm{~g}$ yeast & Gourvita GmbH & $\begin{array}{l}\text { Adam-Opel-Str. 19 } \\
\text { DEU-63322 Rödermark }\end{array}$ \\
\hline $800 \mathrm{~g}$ cornmeal & $\begin{array}{l}\text { ZIELER \& CO. } \\
\text { GmbH }\end{array}$ & $\begin{array}{l}\text { Liebigstraße 101 } \\
\text { DEU-22113 Hamburg }\end{array}$ \\
\hline $220 \mathrm{~g}$ treacle & Obermühle Rosdorf & $\begin{array}{l}\text { Obere Mühlenstraße 3 } \\
\text { DEU-37124 Rosdorf }\end{array}$ \\
\hline $800 \mathrm{~g}$ malt & $\begin{array}{l}\text { MeisterMarken - } \\
\text { Ulmer Spatz }\end{array}$ & $\begin{array}{l}\text { Mainzer Straße 152-160 } \\
\text { DEU-55411 Bingen am Rhein }\end{array}$ \\
\hline
\end{tabular}




\begin{tabular}{|l|l|l|}
\hline $\begin{array}{l}63 \mathrm{~mL} \text { propionic } \\
\text { acid }\end{array}$ & $\begin{array}{l}\text { Carl Roth GmbH + } \\
\text { Co. KG }\end{array}$ & $\begin{array}{l}\text { Schoemperlenstr. 1-5 } \\
\text { DEU-76185 Karlsruhe }\end{array}$ \\
\hline $15 \mathrm{~mL}$ nipagin & $\begin{array}{l}\text { Sigma-Aldrich } \\
\text { Chemie GmbH }\end{array}$ & $\begin{array}{l}\text { Eschenstraße 5 } \\
\text { DEU-82024 Taufkirchen }\end{array}$ \\
\hline $50 \mathrm{~mL}$ ethanol & $\begin{array}{l}\text { VWR International } \\
\text { GmbH }\end{array}$ & $\begin{array}{l}\text { Hilpertstraße 20a } \\
\text { DEU-64295 Darmstadt }\end{array}$ \\
\hline
\end{tabular}

Before cooking, the agar has to be watered overnight in order to dissolve. The following day, soy flour, yeast, cornmeal, treacle, and malt are added while heating the food to $99.5^{\circ} \mathrm{C}$. After cooling down to $55^{\circ} \mathrm{C}$, propionic acid, and nipagin dissolved in ethanol are added. Subsequently, the food is filled into food vials and stored on $4{ }^{\circ} \mathrm{C}$.

\subsubsection{Low-, medium-, and high-calorie food}

The following ingredients are used to make low-, medium-, and high calorie food.

\begin{tabular}{|l|c|c|c|c|}
\hline Ingredient & $\begin{array}{c}\text { Calorie-value } \\
\text { per } 100 \mathrm{~g}[\mathrm{kcal}]\end{array}$ & Low-calorie & Medium-calorie & High-calorie \\
\hline Cornmeal & 356 & $8 \%$ & $8 \%$ & $8 \%$ \\
\hline Agar & 340 & $0.5 \%$ & $0.5 \%$ & $0.5 \%$ \\
\hline Sucrose $*$ & 387 & $2 \%$ & $5 \%$ & $15 \%$ \\
\hline Yeast $* *$ & 127 & $0.25 \%$ & $2 \%$ & $2 \%$ \\
\hline Lard $* * *$ & 898 & - & - & $1 \%$ \\
\hline Propionic acid & 7 & $6.3 \mathrm{~mL} / \mathrm{L}$ & $6.3 \mathrm{~mL} / \mathrm{L}$ & $6.3 \mathrm{~mL} / \mathrm{L}$ \\
\hline $\begin{array}{l}\text { Kilocalories } \\
\text { per } 100 \mathrm{~g}\end{array}$ & & 38 & 52 & 100 \\
\hline
\end{tabular}

* produced by Carl Roth GmbH + Co. KG, Schoemperlenstr. 1-5, DEU-76185 Karlsruhe. ** produced by Deutsche Hefewerke $\mathrm{GmbH}$, Bucher Hauptstr. 53-55, 90427 Nürnberg. *** produced by LARU GmbH, Weusterstraße 25, 46240 Bottrop

After cooking, the food is filled into food vials and stored on $4^{\circ} \mathrm{C}$ until usage.

\subsubsection{Buffers and solutions}

\begin{tabular}{|l|l|l|}
\hline Name & Ingredients & Remarks \\
\hline PBS (phosphate buffered & $15 \mathrm{mM} \mathrm{NaH}_{2} \mathrm{PO}_{4}$ & $\mathrm{HCl}$ or $\mathrm{NaOH}$ are \\
saline) & $100 \mathrm{mM} \mathrm{NaCl}$ \\
& $85 \mathrm{mM} \mathrm{Na}_{2} \mathrm{HPO}_{4}$ & $\begin{array}{l}\text { added to adjust the } \mathrm{pH} \\
\text { to } 7.4\end{array}$ \\
\hline PBST (PBS + Triton $\times 100)$ & $50 \mathrm{~mL} \mathrm{PBS}$ & $\begin{array}{l}\text { Stored in the fridge at } \\
5-8{ }^{\circ} \mathrm{C}\end{array}$ \\
\hline
\end{tabular}




\begin{tabular}{|c|c|c|}
\hline Blocking solution & $\begin{array}{l}1 \mathrm{~mL} \text { PBST } \\
20 \mathrm{mg} \text { albumin }\end{array}$ & $\begin{array}{l}\text { Stored in the fridge at } \\
5-8{ }^{\circ} \mathrm{C}\end{array}$ \\
\hline Ringer's solution & $\begin{array}{l}5 \mathrm{mM} \text { Hepes } \\
130 \mathrm{mM} \mathrm{NaCl} \\
5 \mathrm{mM} \mathrm{KCl} \\
2 \mathrm{mM} \mathrm{CaCl}_{2} \\
2 \mathrm{mM} \mathrm{MgCl} 2 * 2 \mathrm{H}_{2} \mathrm{O} \\
36 \mathrm{mM} \text { sucrose }\end{array}$ & $\begin{array}{l}\mathrm{pH} \text { is adjusted to } 7.4 \\
\text { with } \mathrm{NaOH} \text { and the } \\
\text { solution stored at }-20 \\
{ }^{\circ} \mathrm{C} \text {. }\end{array}$ \\
\hline $\begin{array}{l}4 \% \text { PFA } \\
\text { (Paraformaldehyde) }\end{array}$ & $\begin{array}{l}25 \mathrm{~mL} \text { PBS } \\
1 \mathrm{~g} \text { paraformaldehyde } \\
2.5 \mu \mathrm{L} \mathrm{NaOH}\end{array}$ & $\begin{array}{l}\text { PBS and } \\
\text { paraformaldehyde are } \\
\text { mixed at } 60^{\circ} \mathrm{C} \text {. } \\
\text { The solution is stored } \\
\text { at }-20^{\circ} \mathrm{C} \text {. }\end{array}$ \\
\hline
\end{tabular}

\subsubsection{Antibodies}

\begin{tabular}{|l|l|l|l|l|}
\hline Antigen & $\begin{array}{l}\text { Raised } \\
\text { in }\end{array}$ & Concentration & Fluorophor & Source, catalogue number \\
\hline
\end{tabular}

Primary antibodies

\begin{tabular}{|l|l|l|c|l|}
\hline Nc82 & mouse & $1: 10$ & - & E. Buchner \\
\hline GFP & rabbit & $1: 1000$ & - & Invitrogen $*$, A6455 \\
\hline GFP & chicken & $1: 1000$ & - & Invitrogen *, A11120 \\
\hline
\end{tabular}

Secondary antibodies

\begin{tabular}{|l|l|l|l|l|}
\hline mouse & goat & $1: 300$ & Alexa 568 & Invitrogen $*, A 11004$ \\
\hline rabbit & goat & $1: 500$ & Alexa 488 & Invitrogen $*, A 1104$ \\
\hline chicken & goat & $1: 500$ & Alexa 488 & Invitrogen $*, A 11039$ \\
\hline
\end{tabular}

* Thermo Fisher Scientific GmbH, Im Steingrund 4-6, 63303 Dreieich

\subsubsection{Chemicals \& Consumables}

\begin{tabular}{|l|l|l|}
\hline Name & Company & Address \\
\hline Triton X 100 & $\begin{array}{l}\text { Carl Roth GmbH + } \\
\text { Co. KG }\end{array}$ & $\begin{array}{l}\text { Schoemperlenstr. 1-5 } \\
\text { DEU-76185 Karlsruhe }\end{array}$ \\
\hline
\end{tabular}




\begin{tabular}{|l|l|l|}
\hline Albumin Fraction V & $\begin{array}{l}\text { Carl Roth GmbH + } \\
\text { Co. KG }\end{array}$ & $\begin{array}{l}\text { Schoemperlenstr. 1-5 } \\
\text { DEU-76185 Karlsruhe }\end{array}$ \\
\hline Pipette tips & Sarstedt AG \& Co & $\begin{array}{l}\text { Sarstedtstraße 1 } \\
\text { DEU-51588 Nümbrecht }\end{array}$ \\
\hline $\begin{array}{l}\text { Cover glasses } \\
18 \mathrm{~mm} \times 18 \mathrm{~mm}\end{array}$ & $\begin{array}{l}\text { Thermo Fisher } \\
\text { Scientific GmbH }\end{array}$ & $\begin{array}{l}\text { Im Steingrund 4-6 } \\
\text { DEU-63303 Dreieich }\end{array}$ \\
\hline Cover glasses $\mathrm{mm} \times 60 \mathrm{~mm}$ & $\begin{array}{l}\text { Th. Geyer GmbH \& } \\
\text { Co. KG }\end{array}$ & $\begin{array}{l}\text { Dornierstr. 4-6 } \\
\text { DEU-71272 Renningen }\end{array}$ \\
\hline Microscope slides & $\begin{array}{l}\text { Carl Roth GmbH + } \\
\text { Co. KG }\end{array}$ & $\begin{array}{l}\text { Schoemperlenstr. 1-5 } \\
\text { DEU-76185 Karlsruhe }\end{array}$ \\
\hline Insect pins & $\begin{array}{l}\text { Pin Service - Lucie } \\
\text { Hrabovská }\end{array}$ & $\begin{array}{l}\text { Čsl. Červeného kř́že 967 } \\
\text { CZE-68401 Slavkov u Brna }\end{array}$ \\
\hline Forceps & $\begin{array}{l}\text { Fine Science Tools } \\
\text { GmbH }\end{array}$ & $\begin{array}{l}\text { Vangerowstraße 14 } \\
\text { DEU-69115 Heidelberg }\end{array}$ \\
\hline Food vials & Sarstedt AG \& Co & $\begin{array}{l}\text { Sarstedtstraße 1 } \\
\text { DEU-51588 Nümbrecht }\end{array}$ \\
\hline
\end{tabular}

\subsubsection{Laboratory equipment}

\begin{tabular}{|l|l|l|}
\hline Name & Company & Address \\
\hline Waterbath & $\begin{array}{l}\text { Memmert GmbH }+ \\
\text { Co. KG }\end{array}$ & $\begin{array}{l}\text { Aeussere Rittersbacher Straße 38 } \\
\text { DEU-91126 Schwabach }\end{array}$ \\
\hline Precision scale & Sartorius AG & $\begin{array}{l}\text { Weender Landstrasse 94-108 } \\
\text { DEU-37075 Göttingen }\end{array}$ \\
\hline Pipettes & Eppendorf AG & $\begin{array}{l}\text { Barkhausenweg 1 } \\
\text { DEU-22339 Hamburg }\end{array}$ \\
\hline Incubator & BINDER GmbH & $\begin{array}{l}\text { Im Mittleren Ösch 5 } \\
\text { DEU-78532 Tuttlingen }\end{array}$ \\
\hline Shaker & $\begin{array}{l}\text { GFL Gesellschaft } \\
\text { für Labortechnik } \\
\text { GmbH }\end{array}$ & $\begin{array}{l}\text { Schulze-Delitzsch-Straße 4 } \\
\text { DEU-30938 Burgwedel }\end{array}$ \\
\hline Stereo microscope & $\begin{array}{l}\text { Carl Zeiss AG } \\
\text { Confocal microscope }\end{array}$ & $\begin{array}{l}\text { Leica Microsystems } \\
\text { GmbH }\end{array}$ \\
\hline
\end{tabular}




\begin{tabular}{|l|l|l|}
\hline Objectives & $\begin{array}{l}\text { Leica Microsystems } \\
\text { GmbH }\end{array}$ & $\begin{array}{l}\text { Ernst-Leitz-Strasse 17-37 } \\
\text { DEU-35578 Wetzlar }\end{array}$ \\
\hline Mercury lamp & $\begin{array}{l}\text { Leica Microsystems } \\
\text { GmbH }\end{array}$ & $\begin{array}{l}\text { Ernst-Leitz-Strasse 17-37 } \\
\text { DEU-35578 Wetzlar }\end{array}$ \\
\hline
\end{tabular}

Confocal microscope.

All images were acquired using a Leica TCS SP8 confocal microscope equipped with two photomultipliers and two Leica hybrid detectors. Objectives used were a Leica 10x air PL FLUOTAR objective with a numerical aperture of 0.3 and a Leica 20x glycerol/water PL APO objective with a numerical aperture of 0.75 . For excitation, a $488 \mathrm{~nm}$ argon and a $561 \mathrm{~nm}$ DPSS laser were used.

\subsubsection{Software}

\begin{tabular}{|l|l|l|}
\hline Name & Company & Address \\
\hline $\begin{array}{l}\text { Microsoft Office } \\
\text { Powerd, Excel, }\end{array}$ & $\begin{array}{l}\text { Microsoft } \\
\text { Corporation }\end{array}$ & $\begin{array}{l}\text { One Microsoft Way } \\
\text { USA WA-98052-6399 Redmond }\end{array}$ \\
\hline OriginPro & $\begin{array}{l}\text { OriginLab } \\
\text { Corporation }\end{array}$ & $\begin{array}{l}\text { One Roundhouse Plaza, Suite 303 } \\
\text { USA MA-01060 Northhampton }\end{array}$ \\
\hline Adobe Illustrator & $\begin{array}{l}\text { Adobe Systems } \\
\text { Software Ireland } \\
\text { Limited }\end{array}$ & $\begin{array}{l}\text { 4-6 Riverwalk } \\
\text { Citywest Business Campus } \\
\text { IRL-24 Dublin }\end{array}$ \\
\hline R & $\begin{array}{l}\text { The R Foundation } \\
\text { Felthandelsplatz 1 } \\
\text { AUT-1020 Vienna }\end{array}$ \\
\hline Fiji & GitHub & $\begin{array}{l}\text { 88 Colin P Kelly Jr St } \\
\text { USA CA-94107 San Francisco }\end{array}$ \\
\hline Neurolucida & MBF Bioscience & $\begin{array}{l}\text { 185 Allen Brook Lane, Suite 101 } \\
\text { USA VT-05495 Williston }\end{array}$ \\
\hline Suite (LAS) & $\begin{array}{l}\text { Leica Microsystems } \\
\text { GmbH }\end{array}$ & $\begin{array}{l}\text { Ernst-Leitz-Strasse 17-37 } \\
\text { DEU-35578 Wetzlar }\end{array}$ \\
\hline
\end{tabular}




\subsection{Methods}

\subsubsection{Generation of new transgenic strains}

\subsubsection{Generation of new balancer fly strains}

New balancer lines were generated in order to be able to combine the X-chromosomal inserts of the MARCM system with transgenes on the $2^{\text {nd }}$, and $3^{\text {rd }}$ chromosome. The completed balancer fly strain is highlighted by a frame.

(A)

I $\quad$ † $\frac{h s F l p, t u b P-G a l 80, w-, F R T 19 A}{h s F l p, t u b P-G a l 80, w-, F R T 19 A} ; ; \quad$ X o $\frac{w-}{Y} ; \frac{C y O}{G l a} ; \frac{T M 3}{T M 6}$

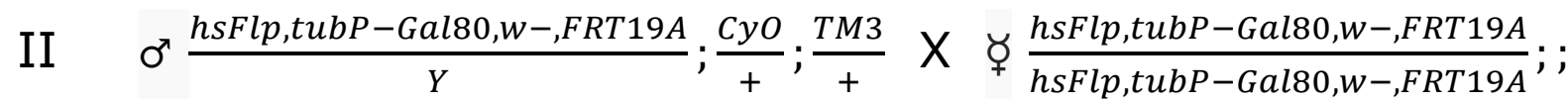

III ఫॄ $\frac{h s F l p, t u b P-G a l 80, w-, F R T 19 A}{h s F l p, t u b P-G a l 80, w-, F R T 19 A} ; \frac{C y O}{+} ; \frac{T M 3}{+}$

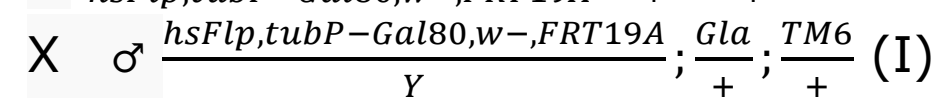

$\Rightarrow \frac{h s F l p, t u b P-G a l 80, w-, F R T 19 A}{h s F l p, t u b P-G a l 80, w-, F R T 19 A} ; \frac{C y O}{G l a} ; \frac{T M 3}{T M 6}$

(B)

I $\quad$ ช $\frac{y, w-, F R T 19 A}{y, w-, F R T 19 A} ; ; \quad$ X $\quad$ o $\frac{w-}{Y} ; \frac{C y O}{G l a} ; \frac{T M 3}{T M 6}$

II $\quad$ ơ $\frac{y, w-, F R T 19 A}{Y} ; \frac{C y O}{+} ; \frac{T M 3}{+} \quad$ X $\quad \succ \frac{y, w-, F R T 19 A}{y, w-, F R T 19 A} ; ;$

III $\Varangle \quad \frac{y, w-, F R T 19 A}{y, w-, F R T 19 A} ; \frac{C y O}{+} ; \frac{T M 3}{+} \quad X \quad$ or $\frac{y, w-, F R T 19 A}{Y} ; \frac{G l a}{+} ; \frac{T M 6}{+}$

$\Rightarrow \frac{y, w-, F R T 19 A}{y, w-, F R T 19 A} ; \frac{C y O}{G l a} ; \frac{T M 3}{T M 6}$

\subsubsection{Generation of new transgenic fly strains for MARCM studies}

Different Gal4 driver lines were used to generate MARCM clones in different subsets of KCs. Therefore, the Gal4 driver lines 5HT1B-Gal4 for the $y$ lobes, GMR35B12-Gal4 for the $a^{\prime} / \beta^{\prime}$ lobes, and OK107-Gal4 for the $a / \beta$ lobes were combined with FRT19A (Xu and Rubin, 1993) on the X-chromosome using the newly generated balancer fly strains 
(see previous section). In cases when homozygous strains were stable balancer insertions were crossed out. The completed fly strain is highlighted by a frame.

(A)

I $\quad \succ \frac{y, w-, F R T 19 A}{y, w-, F R T 19 A} ; \frac{\text { CyO }}{\text { Gla }} ; \frac{\text { TM3 }}{\text { TM6 }} \quad$ X $\quad$ or $\frac{w-}{Y} ; ; \frac{5 H T 1 B-\text { Gal4 }}{5 H T 1 B-\text { Gal4 }}$

II $\quad$ ơ $\frac{y, w-, F R T 19 A}{Y} ; \frac{C y O}{+} ; \frac{T M 3}{5 H T 1 B-G a l 4} \times \quad \succ \frac{y, w-, F R T 19 A}{y, w-, F R T 19 A} ; \frac{\text { CyO }}{\text { Gla }} ; \frac{\text { TM3 }}{\text { TM6 }}$

III $\Varangle \quad \frac{y, w-, F R T 19 A}{y, w-, F R T 19 A} ; \frac{C y O}{+} ; \frac{T M 6}{5 H T 1 B-G a l 4} \times \quad$ or $\frac{y, w-, F R T 19 A}{Y} ; \frac{C y O}{+} ; \frac{T M 3}{5 H T 1 B-G a l 4}$

$\Rightarrow \frac{y, w-, F R T 19 A}{y, w-, F R T 19 A} ; \frac{+}{+} ; \frac{5 H T 1 B-G a l 4}{5 H T 1 B-G a l 4}$

The $3^{\text {rd }}$ chromosomal driver line GMR35B12-Gal4 (Jenett et al., 2012) was crossed the same way as shown above, leading to:

$\Rightarrow \frac{y, w-, F R T 19 A}{y, w-, F R T 19 A} ; \frac{+}{+} ; \frac{\mathrm{GMR} 35 \mathrm{~B} 12-\mathrm{Gal} 4}{\mathrm{GMR} 35 \mathrm{~B} 12-\mathrm{Gal} 4}$

Further, OK107-Gal4 (Connolly et al., 1996) located on the $4^{\text {th }}$ chromosome was combined to FRT19A ( $\mathrm{Xu}$ and Rubin, 1993) on the X-chromosome using the $\mathrm{ci}^{\mathrm{D}}$ mutation (Von Ohlen and Hooper, 1999), which is also located on the $4^{\text {th }}$ chromosome:

(B)

I $\quad$ † $\frac{y, w-, F R T 19 A}{y, w-, F R T 19 A} \quad \mathrm{X} \quad$ ơ $\frac{w-}{Y} ; ; ; \frac{\text { OK107-Gal4 }}{c i D}$

II $\quad$ or $\frac{y, w-, F R T 19 A}{Y} ; ; ; \frac{+}{c i D} \quad \mathrm{\varnothing} \quad \Varangle \frac{y, w-, F R T 19 A}{y, w-, F R T 19 A}$

III $\quad \Varangle \quad \frac{y, w-, F R T 19 A}{y, w-, F R T 19 A} ; ; ; \frac{+}{c i D} \quad X \quad$ or $\frac{y, w-, F R T 19 A}{Y} ; ; ; \frac{+}{\text { OK107-Gal4 }}$

$\Rightarrow \frac{y, w-, F R T 19 A}{y, w-, F R T 19 A} ; ; ; \frac{\text { OK107-Gal4 }}{\text { OK107-Gal4 }}$

The UAS reporter line 6XmCherry (Shearin et al., 2014) and the green calcium sensor GCaMP 3.0 (Tian et al., 2009) targeted to the MBs via the MB247 promoter (Pech et al., 2013a; Riemensperger et al., 2005), both used in this study as MB background markers, were combined with hsFlp, FRT19A, tubP-Gal80 (Golic, 1991; Lee et al., 1999; Xu and Rubin, 1993) the following way: 
Ia $\Varangle \frac{h s F l p, t u b P-G a l 80, w-, F R T 19 A}{h s F l p, t u b P-G a l 80, w-, F R T 19 A} ; \frac{\text { CyO }}{\text { Gla }} ; \frac{\text { TM3 }}{\text { TM6 }}$ X ơ $\frac{w-}{Y} ; \frac{M B 247-G C a M P 3.0}{G l a}$;

Ib $\Varangle \frac{h s F l p, t u b P-G a l 80, w-, F R T 19 A}{h s F l p, t u b P-G a l 80, w-, F R T 19 A} ; \frac{\text { CyO }}{\text { Gla }} ; \frac{\text { TM3 }}{\text { TM6 }}$

$\mathrm{X} \quad \sigma^{\circ} \frac{w-}{Y} ; \frac{S p}{C y O, W e e-P} ; \frac{20 \mathrm{XUAS}-6 \mathrm{XmCherry}}{20 \mathrm{XUAS}-6 \mathrm{XmCherry}}$

IIa $\sigma^{7} \frac{h s F l p, t u b P-G a l 80, w-, F R T 19 A}{Y} ; \frac{M B 247-G C a M P 3.0}{G l a} ; \frac{T M 3}{+}$

X ఫ̛ $\frac{h s F l p, t u b P-G a l 80, w-, F R T 19 A}{h s F l p, t u b P-G a l 80, w-, F R T 19 A} ; \frac{\text { CyO }}{\text { Gla }} ; \frac{\text { TM3 }}{\text { TM6 }}$

III $\Varangle \frac{h s F l p, t u b P-G a l 80, w-, F R T 19 A}{h s F l p, t u b P-G a l 80, w-, F R T 19 A} ; \frac{M B 247-G C a M P 3.0}{C y O} ; \frac{T M 3}{T M 6}$

$\mathrm{X} \sigma \frac{h s F l p, t u b P-G a l 80, w-, F R T 19 A}{Y} ; \frac{G l a}{C y O, W e e-P+} ; \frac{20 \mathrm{XUAS}-6 \mathrm{XmCherry}}{T M 3}$

$\Rightarrow \frac{h s F l p, t u b P-G a l 80, w-, F R T 19 A}{h s F l p, t u b P-G a l 80, w-, F R T 19 A} ; \frac{M B 247-G C a M P 3.0}{C y O, W e e-P} ; \frac{20 X U A S-6 X m C h e r r y}{20 X U A S-6 X m C h e r r y}$

To combine UAS:brpshort::GFP (Fouquet et al., 2009) on the $3^{\text {rd }}$ chromosome and 20XUAS-6XmCherry on the $2^{\text {nd }}$ chromosome with hsFlp, tubP-Gal80, $w^{-}$, FRT19A on the $\mathrm{X}$-chromosome, the same crossing strategy was used, leading to:

$\Rightarrow \frac{h s F l p, t u b P-G a l 80, w-, F R T 19 A}{h s F l p, t u b P-G a l 80, w-, F R T 19 A} ; \frac{20 \mathrm{XUAS}-6 \mathrm{XmCherry}}{G l a} ; \frac{\mathrm{UAS}: \text { brp }^{\wedge} \text { short::GFP }}{\mathrm{TM} 3}$

\subsubsection{Fly husbandry}

All flies were reared in incubators in constant $12 \mathrm{~h} / 12 \mathrm{~h}$ light-dark cycles on $25^{\circ} \mathrm{C}$ and $60 \%$ humidity on standard cornmeal food. In experiments investigating structural plasticity of KCs in flies raised on different calorie foods, the flies were aged 7-9 days when used for analysis. In those experiments flies aged up to 1 day were placed on either low-calorie, medium-calorie, or high-calorie food for 7-8 days before being dissected for confocal imaging. In all other experiments, flies were aged 4-7 days. Female flies were used throughout. All flies used, were age controlled by allowing parental lines to lie eggs in a short time window of 2-8 hours.

The following fly lines were crossed to generate transgenic lines for MARCM:

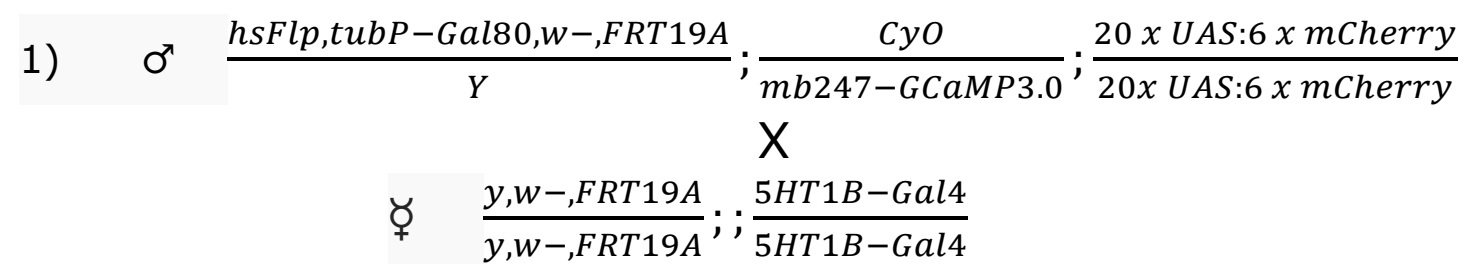


Leading to flies of the F1 generation that potentially express 6XmCherry in single $Y$ lobe Kenyon cells:

은 $\frac{h s F l p, \text { tubP-Gal80,w-,FRT19A }}{y, w-, F R T 19 A} ; \frac{+}{m b 247-G C a M P 3.0} ; \frac{5 H T 1 B-G a l 4}{20 x \text { UAS:6 x mCherry }}$

2) $\sigma^{7} \frac{\text { hsFlp,tubP-Gal80,w-,FRT19A }}{Y} ; \frac{C y O}{m b 247-G C a M P 3.0} ; \frac{20 \times U A S: 6 \times \text { mCherry }}{20 \times U A S: 6 \times \text { mCherry }}$ $\mathrm{X}$

$$
\Varangle \quad \frac{y, w-, F R T 19 A}{y, w-, F R T 19 A} ; ; \frac{G M R 35 B 12-G a l 4}{G M R 35 B 12-G a l 4}
$$

Leading to flies of the F1 generation that potentially express $6 \mathrm{XmCherry}$ in single $\mathrm{a}^{\prime} / \beta^{\prime}$ lobe Kenyon cells:

ㅇ $\frac{h s F l p, \text { tubP-Gal80,w-,FRT19A }}{y, w-, F R T 19 A} ; \frac{+}{m b 247-G C a M P 3.0} ; \frac{\text { GMR35B12-Gal4 }}{20 x \text { UAS:6 } x \text { mCherry }}$

3) $\sigma^{\pi} \frac{h s F l p, t u b P-G a l 80, w-, F R T 19 A}{Y} ; \frac{C y O}{m b 247-\text { GCaMP } 3.0} ; \frac{20 \times \text { UAS:6 } x \text { mCherry }}{20 x \text { UAS:6 } x \text { mCherry }}$

$$
\text { ఫ } \quad \frac{y, w-, F R T 19 A}{y, w-, F R T 19 A} ; ; ; \frac{O K 107-G a l 4}{O K 107-G a l 4}
$$

Leading to flies of the F1 generation that potentially express 6 XmCherry in single $a / \beta$ lobe Kenyon cells:

인 $\frac{h s F l p, t u b P-G a l 80, w-, F R T 19 A}{y, w-, F R T 19 A} ; \frac{+}{m b 247-G C a M P 3.0} ; \frac{+}{20 x \text { UAS:6 x mCherry }} ; \frac{+}{\text { OK107-Gal4 }}$

\subsubsection{MARCM dependent single cell clone generation by heat shock}

In order to induce heat-shock promoter dependent flippase (flp) expression (Golic, 1991), the food vials containing the respective larvae were placed in a water bath heated to $37^{\circ} \mathrm{C}$ using a custom build device to keep the vials below the surface. The heat-shock was applied for 2-4 hours interrupted by several breaks of 15-30 minutes on room temperature to avoid overheating of the larvae. Dependent on the type of $\mathrm{KC}$ clone that was to be induced by MARCM the larvae were heat shocked at different time points after hatching. The publication of Lee et al., 1999 was taken as a reference to decide when to apply the heat shock for labeling of the respective cell type (see illustration in figure 3.4 for overview of MB development in respect to the organisms development). 


\subsubsection{Immunohistochemistry}

Fly brains were dissected in ice cold Ringer's solution using fine forceps. After the dissection, the brains were kept in Ringer's solution until all brains of the set of flies were dissected. Subsequently, the brains were placed in $4 \%$ PFA for 1.5 hours on the shaker in $4{ }^{\circ} \mathrm{C}$. Brains were then rinsed three times for 20 minutes in $0.6 \%$ PBST, incubated for 2 hours at room temperature in blocking solution, and then incubated for 2 days on $4^{\circ} \mathrm{C}$ in the first antibodies solved in blocking solution. After washing, three times 20 minutes in $0.6 \%$ PBST, the brains were incubated in the second antibodies solved in PBST for 3 hours at room temperature. Then, brains were rinsed again two times for 20 minutes in $0.6 \%$ PBST and one time 30 minutes in PBS. In the last step, the brains were mounted in Vectorshield ${ }^{\mathrm{TM}}$ on cover slips that were placed on object holders, and subsequently imaged using confocal microscopy. In case antibody staining was not required, brains were washed after fixation for two times 30 minutes in $0.6 \%$ PBST and one time 30 minutes in PBS before being mounted cover slips.

\subsubsection{Confocal microscopy}

Image acquisition was performed using a Leica SP8 microscope controlled by the Leica produced software LCA. Samples were focused using a 10x air objective and subsequently scanned using a 20x glycerol/water (with the ratio 80/20 in the immersion medium used) objective. For fluorophore excitation, the argon laser at 488 $\mathrm{nm}$ and the DPSS laser at $561 \mathrm{~nm}$ wavelength were used in parallel. The equipped Leica hybrid detectors (HyDs) were employed for light detection. Laser power, gain, and scanning speed were adjusted to the samples fluorescence intensity leading to a pixel dwell time of $400-600$ ns at a resolution of $1024 \times 1024$. The pinhole size was kept constant at 1 airy unit.

\subsubsection{Image processing}

Confocal acquired images were processed using the open source software Fiji (Schindelin et al., 2012). If not stated otherwise, maximum projections were made from confocal stacks focusing on the cell regions of interest. The resulting maximum projections were improved by adjusting brightness and contrast. Scale bars were added using the standard Fiji plugin. To count the number of labeled cell bodies, the stack counter plugin of Fiji was used that allows to manually set markers on visually identified cell bodies, while scrolling through the $z$ stack using the mouse wheel. 


\subsubsection{Kenyon cell reconstructions}

Kenyon cell morphologies were reconstructed manually using the software Neurolucida $\Re$. The reconstruction was performed using the simple click tracing function. Skeletons of Kenyon cells were traced only, ignoring different volumes along the neurites. Using the background marker expression (MB247-GCaMP3.0) for orientation, MB sub-structures were identified. Accordingly, different parts of single KCs were assigned to the calyx, the peduncle, and the lobes, which in turn were further subdivided in the different compartments described in (Aso et al., 2014a; Tanaka et al., 2008); and illustrated in figure 2.1 for the $y$ lobes. $Y$ neurons fall into compartments $\gamma 1-5, a / \beta$ neurons into $a 1-3$ and $\beta 1-2$, and $a^{\prime} / \beta^{\prime}$ neurons into $a^{\prime} 1-3$ and $\beta^{\prime} 1-2$. The length of the arborizations and the number of nodes were measured within the different cell subdivisions. Moreover, dendritic claws within the calyx were marked and quantified. In case the image quality of a KC was considered to be very good, all bouton-like varicosities along the $\mathrm{KC}$ axons in the lobes were quantified. The primary node of a $\mathrm{Y}$ lobe Kenyon cell was defined within one of the five $y$ lobe compartments according to the following criteria taking into account the complexity of the arborizations. If after a bifurcation both branches will cross at least two compartment borders the node at the bifurcation is considered to be a primary node. In the case of a bifurcation in the $\mathrm{Y} 4$ compartment, the node at the bifurcation is

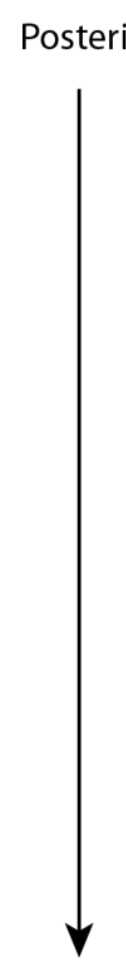

Anterior
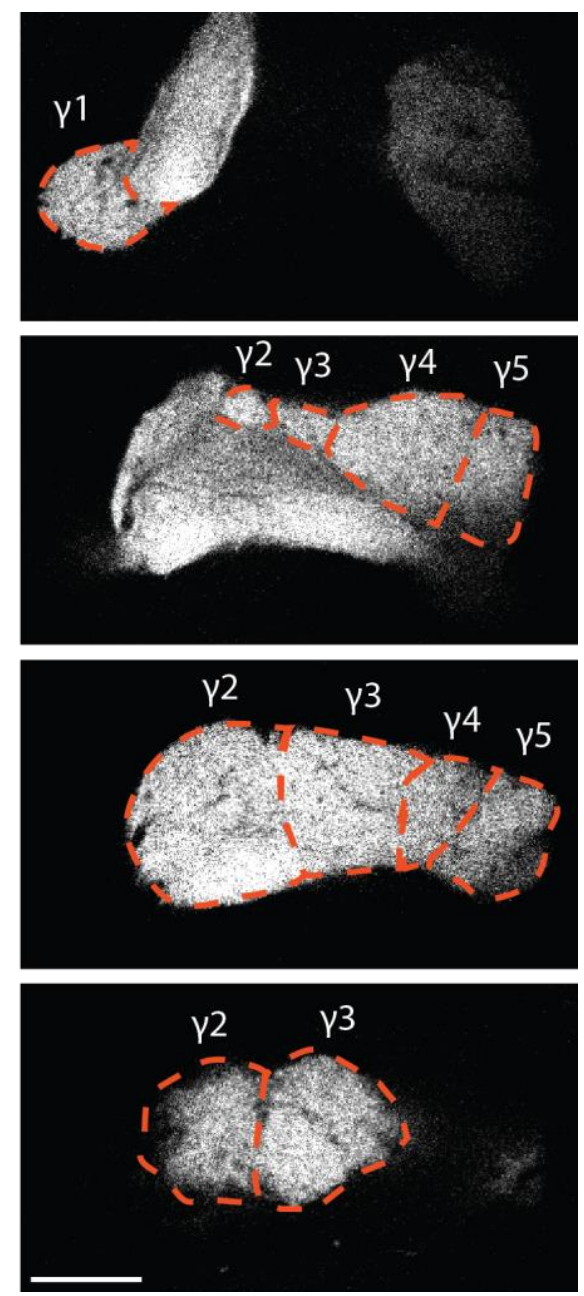

Figure 2.1. Morphological identification of $y$ lobe compartments.

To distinguish different $\mathrm{y}$ lobe compartments, it is necessary to recognize the borders between the compartments. Here, the emergence of the five $y$ lobe compartments, while going through the image stack from the posterior side of the mushroom body lobes to the anterior side. MB247-GCaMP is used as a mushroom body marker. 
both branches crossed the border to the $\mathrm{y} 5$ compartment and were of the same magnitude.

In case of the analysis of $a / \beta$ in the context of different calorie food conditions, $a / \beta$ surface neurons were reconstructed only, in order to avoid bias within the data set.

\subsubsection{Cluster analysis and principal component analysis}

Hierarchical cluster analysis was performed using the function hclust in R (R project, version 3.2.0). As input data Euclidean distances between all pairs of KCs within the respective data set were used based on the variables chosen for the particular analysis. All data were scaled by dividing each entry by the standard deviation of the respective variable. As a criterion for grouping cells into clusters a modified variant of Ward's method of minimum variance (Ward, 1963) implemented as ward.D2 (Murtagh and Legendre, 2014) in R was applied. Ward's method of minimum variance pairs those kind of data points or clusters that lead to the least increase of within cluster variance after merging. Dendrograms were plotted using the package ggdendro.

Principle component analysis was performed using the prcomp function in R. Data were scaled by dividing each entry by the standard deviation of the respective variable. In order to plot KCs in principal component space, the adjusted values were copied to OriginPro 8.5 and a 3D dot plot was generated based on the first three principal components. Coloration was added in OriginPro 8.5 according to the time of MARCM induction or dependent on the cluster affiliation.

\subsubsection{Kenyon cell silhouettes}

Silhouette values are used to describe how well the elements of a cluster fit into it. If $\mathrm{KC} \mathrm{A}$ is an element of cluster $\mathrm{C} 1$ and $\mathrm{C} 2$ is the neighboring cluster to $\mathrm{C} 1$, then the silhouette value gives a measure of the cells relative distance to $\mathrm{C} 1$ in comparison to C2. More precisely, if $\mathrm{c} 1(\mathrm{a})$ is the average Euclidean distance of KC A to all other cells in cluster $\mathrm{C} 1$ and $\mathrm{C2}(\mathrm{a})$ is the average Euclidean distance of KC A to all cells in cluster $\mathrm{C} 2$ then the silhouette value $\mathrm{s}(\mathrm{a})$ of cell $\mathrm{A}$ is defined as

$s(a)=\frac{\mathrm{c} 2(\mathrm{a})-\mathrm{c} 1(\mathrm{a})}{\max \{c 1(a), c 2(a)\}}$

The difference between $c 1(a)$ and $c 2(a)$ is normalized to the maximum of the two values, so that the silhouette values lies between 1 and -1.1 meaning an exact fit to the own cluster, -1 an exact fit to the neighboring cluster. In figure 3.13, 3.14, and 3.19 no negative values are shown but the direction of the bar indicates the relative location of the cell between the two neighboring clusters illustrated by color. 


\subsubsection{Weight measurements}

Weights of flies were measured using a precision scale. To this end, groups of 4 to 17 flies that had spent 7-8 days on special food conditions were transferred into microtubes that were weighed with and without the flies. The difference was then divided by the number of flies to determine the flies' average weight. The average weight was determined before and after the flies have been set on special food conditions and the gain in weight calculated.

\subsubsection{Statistics}

Data comparisons were performed using statistical software OriginPro 8.5 and R. Independent pairs of data were compared using Student's $t$-test if data were normally distributed and Mann-Whitney $U$ test if data were not normally distributed. Normality distribution was determined by the Shapiro-Wilk test. If multiple pairs were compared within the same experiment the p-values were corrected according to Bonferroni's principle. In case that more than two distributions were compared, significance was tested using an analysis of variance (ANOVA) if data were normally distributed or a Kruskal-Wallis test if date were not normally distributed. P-values were corrected for multiple comparisons. Linear regressions were calculated in OriginPro 8.5 using minimum chi-square estimations. To indicate significant differences in figures, $*$ is defined as $\mathrm{p}<0.05$, ** as $\mathrm{p}<0.01$, and $* * *$ as $\mathrm{p}<0.001$. 


\section{Results}

\subsection{Establishing single cell anatomy of mushroom body Kenyon cells}

Single cell analysis of KCs offers the opportunity to resolve the question how exactly they contribute to adaptive behavioral responses, mainly including learning and memory tasks. The memory trace of olfactory associative learning could be pinpointed to KCs using behavioral genetics. Different roles in memory formation, consolidation, and retrieval could be assigned to different subtypes of KCs that are defined based on their gene expression patterns using distinct Gal4 driver lines. However, it remains poorly understood how information is stored at the single $\mathrm{KC}$ level and if there are different morphological or functional types of lobe-specific KCs within identified KC subsets that are specialized to certain behavioral tasks. Therefore, a high number of single KCs shall be stained and analyzed to answer the question of uniformity versus diversity in terms of morphology, and lay the foundation for future functional research. To begin with, three Gal4 driver lines were chosen that express Gal4 in one of the three main KC lobes, and tested for their suitability to be used in genetic mosaic techniques. The Gal4 driver lines chosen were 5HT1B-Gal4 (Yuan et al., 2005), expressing Gal4 in Y KCs of the MB, MB247-Gal4 (Schulz et al., 1996), expressing Gal4 in $y \mathrm{KCs}$ and $\mathrm{a} / \beta \mathrm{KCs}$, and GMR35B12-Gal4 (Jenett et al., 2012), expressing Gal4 in $a^{\prime} / \beta^{\prime}$ KCs (figure 3.1A-C).

\subsubsection{A Flp-out technique to label individual Kenyon cells}

In order to reduce the Gal4 mediated expression of fluorescent proteins down to the single cell level, the Flp-out technique (Harrison and Perrimon, 1993; Struhl and Basler, 1993) was employed. The genetic construct UAS>stop>mcd8-GFP (Wong et al., 2002; Yu et al., 2010; Zhu et al., 2003) includes a transcriptional stop that is flanked by two FRT sites. Only upon excision of the stop cassette by Flp activity mcd8GFP is expressed. In the Flp-out system the Flp is expressed under the control of a heat shock promoter (Golic, 1991). Here, however, no heat shock was applied to 

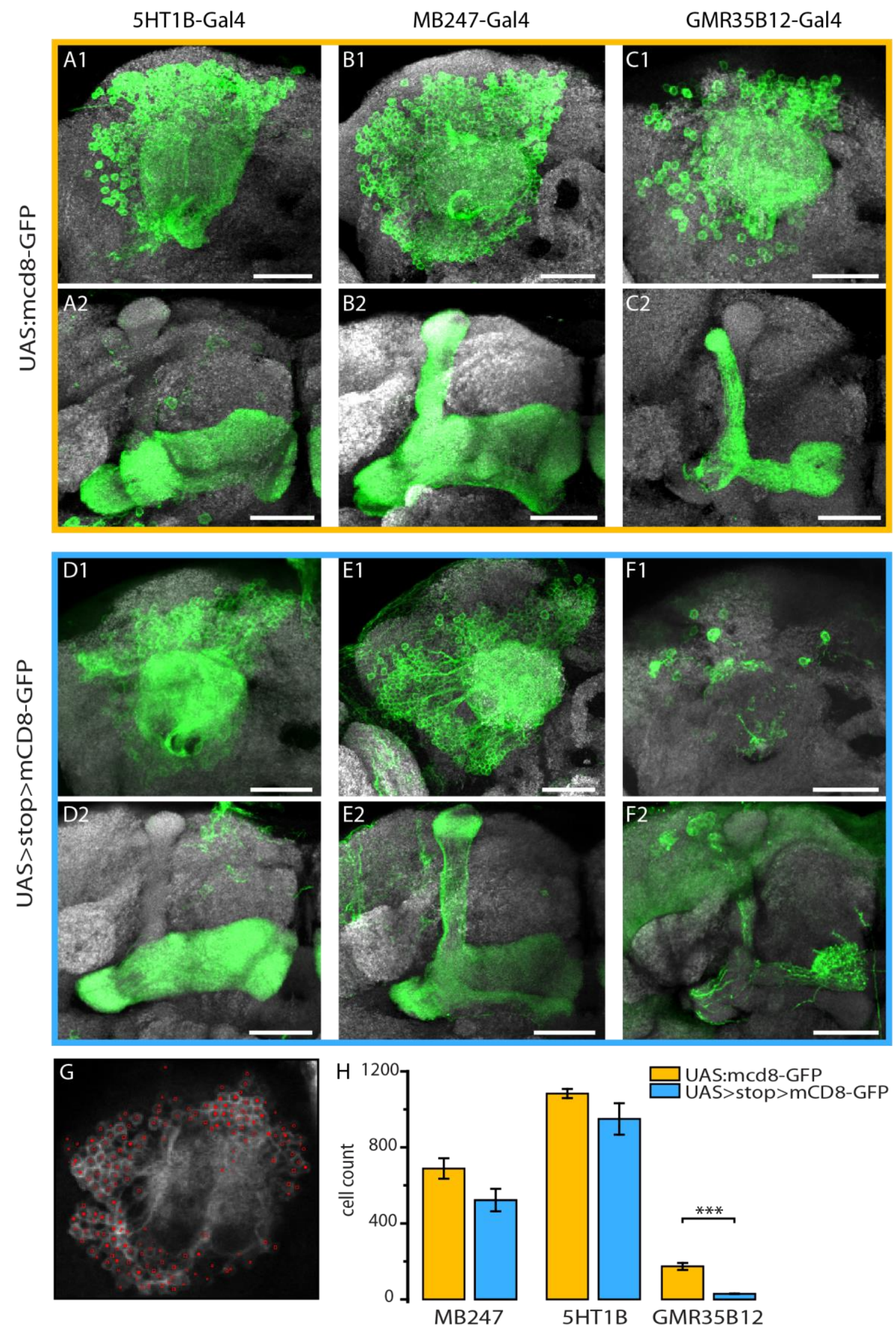

Figure 3.1. Sparse labeling of Kenyon cells using Flp-out.

In order to label single mushroom body Kenyon cells, the Flp-out approach was used to reduce expression of mCD8-GFP by excision of the FRT flanked transcription stop by Flp recombinase activity. Tissue or cell type specific marker expression was achieved by the use of different Gal4 driver lines.

Figure 3.1 continued on next page. 
induce heat shock promoter activity, because Flp-mediated GFP expression was observed even without heat shock.

To evaluate the effectiveness of the Flp-out system in generating single cell clones, UAS>stop>mcd8-GFP was crossed to three different Gal4 driver lines, 5HT1B-Gal4 ( $Y$ lobe), MB247-Gal4 ( $\gamma$ and $a / \beta$ lobes), and GMR35B12-Gal4 ( $\alpha^{\prime} / \beta^{\prime}$ lobes; figure 3.1DF). The number of GFP positive cell bodies with Flp-out and with UAS:mcd8-GFP as a control was counted using the stack counter plugin in FIJI (Schindelin et al., 2012; figure 3.1G). A significant reduction in cell number was observed in GMR35B12-Gal4 $>$ UAS > stop>mcd8-GFP compared to the non Flp-out control, but not in 5HT1B-Gal4 > UAS>stop>mcd8-GFP, and MB247-Gal4 > UAS>stop>mcd8-GFP compared to the respective control (figure $3.1 \mathrm{H}$ ). The cell number in GMR35B12-Gal4 > UAS > stop>mcd8-GFP was reduced by roughly $80 \%$ compared to the UAS:mcd8-GFP control. However, restricted GFP expression in an average of $\sim 30$ cells was not sufficient to distinguish single cell arborizations. Thus, the Flp-out technique in this case, is not suited to reduce marker expression to the single cell level in the MBs.

\subsubsection{Generation of genetic mosaics through MARCM}

Mosaic analysis with a repressible cell marker (MARCM; Lee and Luo, 1999) has been proven in several studies to successfully generate single cell clones in various cell types of the Drosophila nervous system including KCs (Bushey et al., 2011; FurukuboTokunaga et al., 2016; Jefferis et al., 2001; Lee et al., 1999; Scott et al., 2002; Tessier and Broadie, 2008; Zhu et al., 2003). The genetic mosaic method MARCM is based on the flp/FRT induced recombination between homologous chromosomes heterozygous for the Gal4 repressor protein Gal80 (Lee and Luo, 1999; Ma and Ptashne, 1987) during mitotic cell division. If recombination happens during mitosis, subsequent cell lineages will be partly homozygous with and partly homozygous without the repressor.

A-C) Expression patterns of 5HT1B-Gal4 (A), MB247-Gal4 (B), and GMR35B12-Gal4 (C) visualized by mcd8-GFP. In $A 1$ to $C 1$ the calical arborizations and in $A 2$ to $C 2$ the lobal arborizations are shown. 5HT1B-Gal4 drives in the $\gamma$-lobes, MB247-Gal4 in the $\gamma$ - and a/ $\beta$-lobes, and GMR35B12-Gal4 in the $\alpha^{\prime} / \beta^{\prime}$-lobes.

D-F) Gal4 driver lines are combined to UAS>stop>mcd8-GFP, and hs-Flp. No heatshock was applied, flies were raised on $18^{\circ} \mathrm{C}$. Expression patterns of 5HT1B-Gal4 (D), MB247-Gal4 (E), and GMR35B12-Gal4 (F) are shown in the calyx (D1-F1) and lobes (D2-F2).

$\mathrm{G})$ Example of quantification of cell number in \#5HT1b-Gal4 using the stack counter plugin of FIJI.

H) Quantification of cell number in 5HT1B-Gal4, MB247-Gal4, and GMR35B12-Gal4 crossed to UAS: mcd8-GFP (yellow) or to the flp cassette UAS>stop>mcd8-GFP (blue). The mean (columns) and SEM (whiskers) is shown. $\mathrm{N}=2-6$. $* * *$ indicate significance $\mathrm{p}<0.001$, Student's $t$ test, followed by post hoc Bonferroni correction for multiple comparisons. 
Cells missing the repressor will express the reporter, e.g. mCherry, via Gal4/UAS. KCs are generated from four neuroblasts that all produce the same number of KCs from all KC subtypes (Ito et al., 1997; Lee et al., 1999; Zhu et al., 2003). Single KC labeling will occur if Flp-induced recombination affects a dividing ganglion mother cell, the progenitor cell of two KCs, and no other recombination is induced elsewhere. Flp expression is regulated by hsp70 (Ashburner and Bonner, 1979) and hence inducible by heat shock. Dependent on the cell type that is supposed to be labeled, a short heat shock of around 2 hours on $37^{\circ} \mathrm{C}$ is applied around the time of development of the respective cell type (for protocol see section 2.2.3 and Wu and Luo [2006]).

anti-GFP/anti-RFP
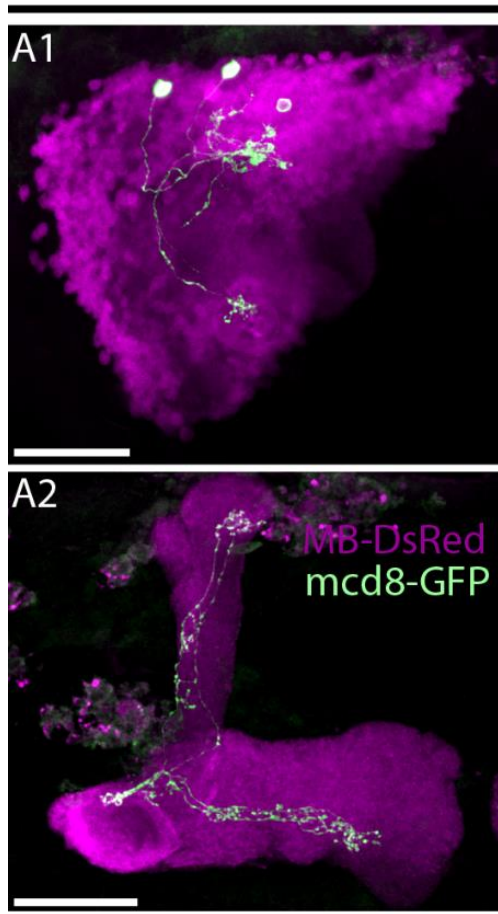

no antibody staining
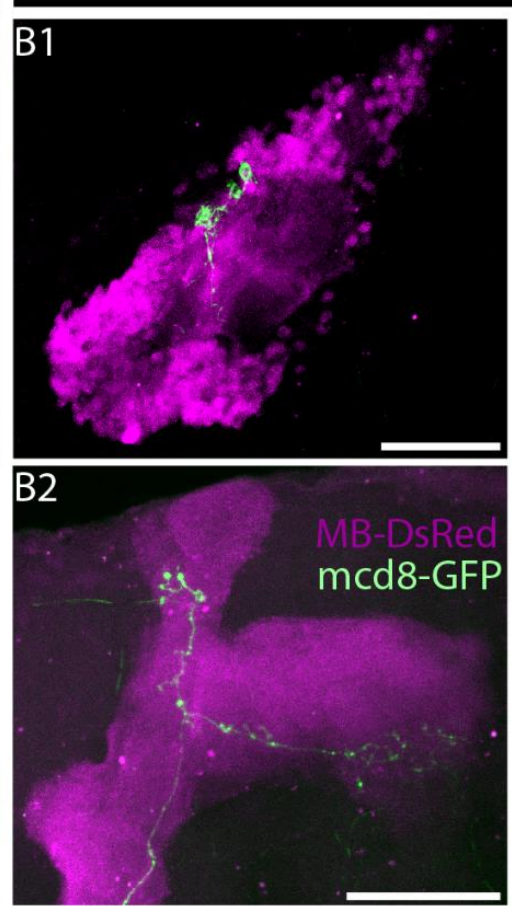
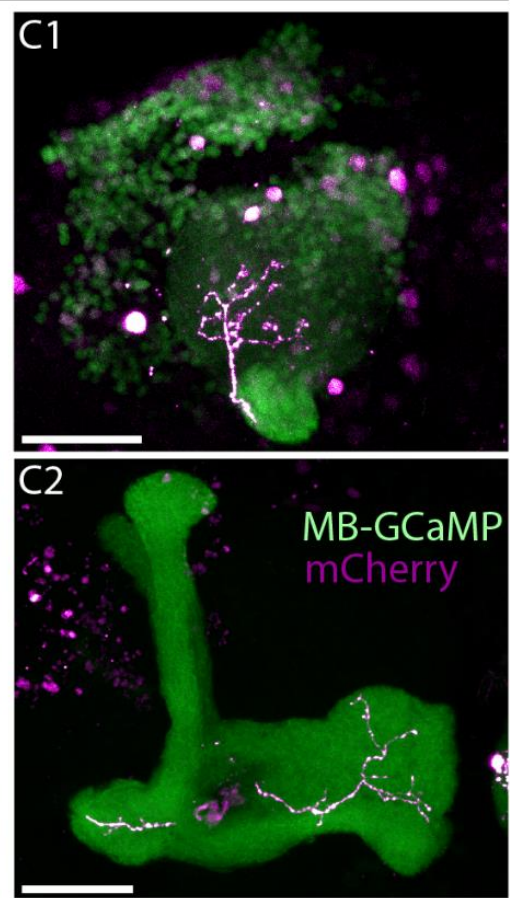

Figure 3.2. Establishing MARCM in Kenyon cells.

In order to label single Kenyon cells using MARCM different cell marker/background marker combinations were tried. mCherry in Kenyon cells and MB247-GCaMP in the mushroom bodies show a more precise labeling profile than mcd8-GFP in Kenyon cells and MB247-DsRed in the mushroom bodies.

A) Sparse expression of mcd8-GFP in three $a / \beta$ posterior KCs by MARCM with OK107 to drive Gal4 and MB-DsRed used as a background marker. Fluorescence was amplified by anti-GFP and anti-RFP antibody staining.

$B$ ) Expression of mcd8-GFP in a single $a^{\prime} / \beta^{\prime} K C$ using the same genotype as in $A$ in the absence of additional antibody staining. The sample was fixed in PFA.

C) Expression of hexameric mCherry in a single $\mathrm{Y}$ lobe $\mathrm{KC}$ with 5HT1B to drive Gal4 and MB247GCaMP as a background marker. The cell is slightly damaged. As in B the sample was fixed in PFA but no additional antibody staining was performed.

A1) to $C 1$ ) expression pattern in the calices, scale bar $=30 \mu \mathrm{m}$.

A2) to $\mathrm{C2}$ ) expression patern in the lobes, scale bar $=30 \mu \mathrm{m}$.

To induce MARCM dependent fluorescence expression a heat shock was applied to all lines during development. Experiments were performed in parallel. 
FRT sites for Flp-mediated mitotic recombination were introduced on the $X, 2^{\text {nd }}$, and $3^{\text {rd }}$ chromosome (Xu and Rubin, 1993). Here, the X-chromosomal FRT19A (Xu and Rubin, 1993) recombination site was used, and combined with either MB247-DsRed (Riemensperger et al., 2005) or MB247-GCaMP (Pech et al., 2013a) as a background marker that can be used to identify MB subdivisions and the compartmental structure of the lobes. To report MARCM events, mcd8-GFP under control of 10 copies of UAS (Pfeiffer et al., 2010) or a hexameric mCherry protein under control of 20 copies of UAS (Shearin et al., 2014) were used. Hexameric mCherry was combined with MBGCaMP as a background, and mcd8-GFP with MB-DsRed as background. Indeed, single $\mathrm{KC}$ labeling could be detected with both combinations. Detection levels of mcd8-GFP in KCs and DsRed in the MBs were appropriately strong if natural fluorescence was amplified using anti-GFP and anti-RFP antibody staining (figure 3.2A). However, in the absence of antibody-mediated signal amplification visibility of cell compartments and single cell arborizations was strongly reduced (figure 3.2B). When hexameric mCherry was expressed in KCs and GCaMP in the MBs the fluorescence signal was robust, allowing analysis and identification of MB compartments and $\mathrm{KC}$ arborizations without
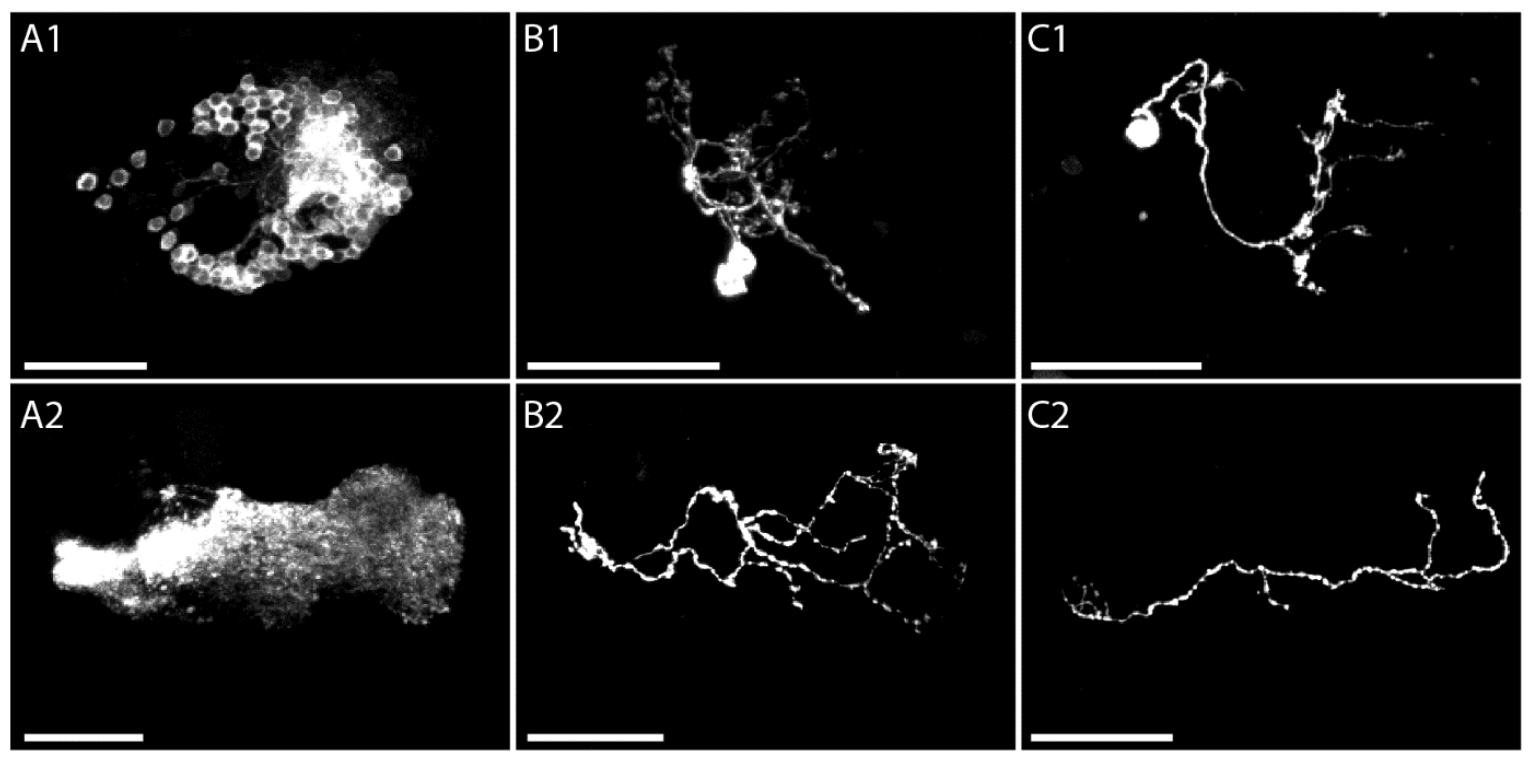

Figure 3.3. Examples of MARCM clones in the $y$ lobe.

The number of clones labeled using MARCM is dependent on Flp expression and mitotic activity of the MB neuroblasts and ganglion mother cells. Therefore, several outcomes regarding the number of labeled cells by MARCM had been observed. Hexameric mCherry was used as a reporter and 5HT1B-Gal4 as a driver line.

A) Labeling of multi cell clones due to absence of repressor protein expression in a MB neuroblast shown in the calyx (A1) and lobes (A2).

B) Labeling of a two cell clone due to absence of repressor protein expression in a ganglion mother cell after MB neuroblast division shown in the calyx (B1) and the lobes (B2).

C) Labeling of a single cell clone due to absence of repressor protein expression in a single KC after division of the ganglion mother cell shown in the calyx (C1) and the lobes (C2).

Scale bars $=20 \mu \mathrm{m}$. 
further antibody amplification (figure 3.2C). Thus, the combination mCherry/GCaMP was preferred over GFP/DsRed to study KC single cell morphologies because it allows for a high experimental throughput while providing a good optical resolution. In addition, the extensive staining procedure and unspecific binding of antibodies might distort the original cell morphology.

Y KCs have been shown to be a highly interesting $\mathrm{KC}$ subset due to their complex role in appetitive and aversive behaviors (e.g. Cohn et al., 2015; Perisse et al., 2016). To describe $\mathrm{Y}$ KC morphologies in detail MARCM was used to generate a set of 75 y KCs

A
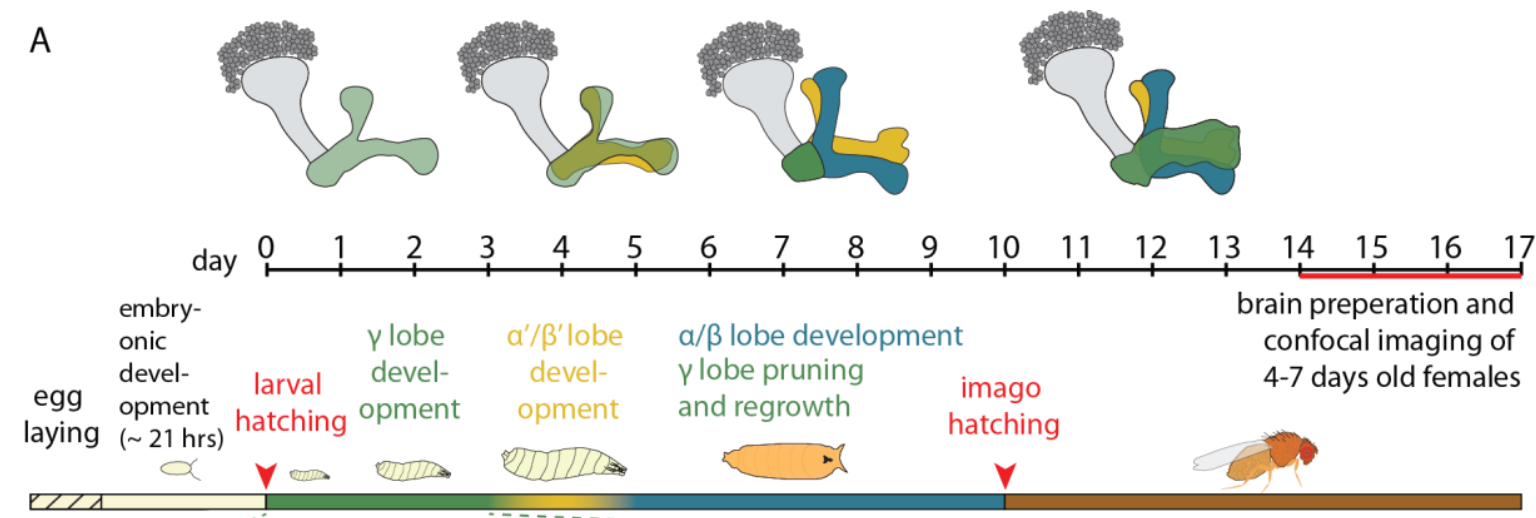

B

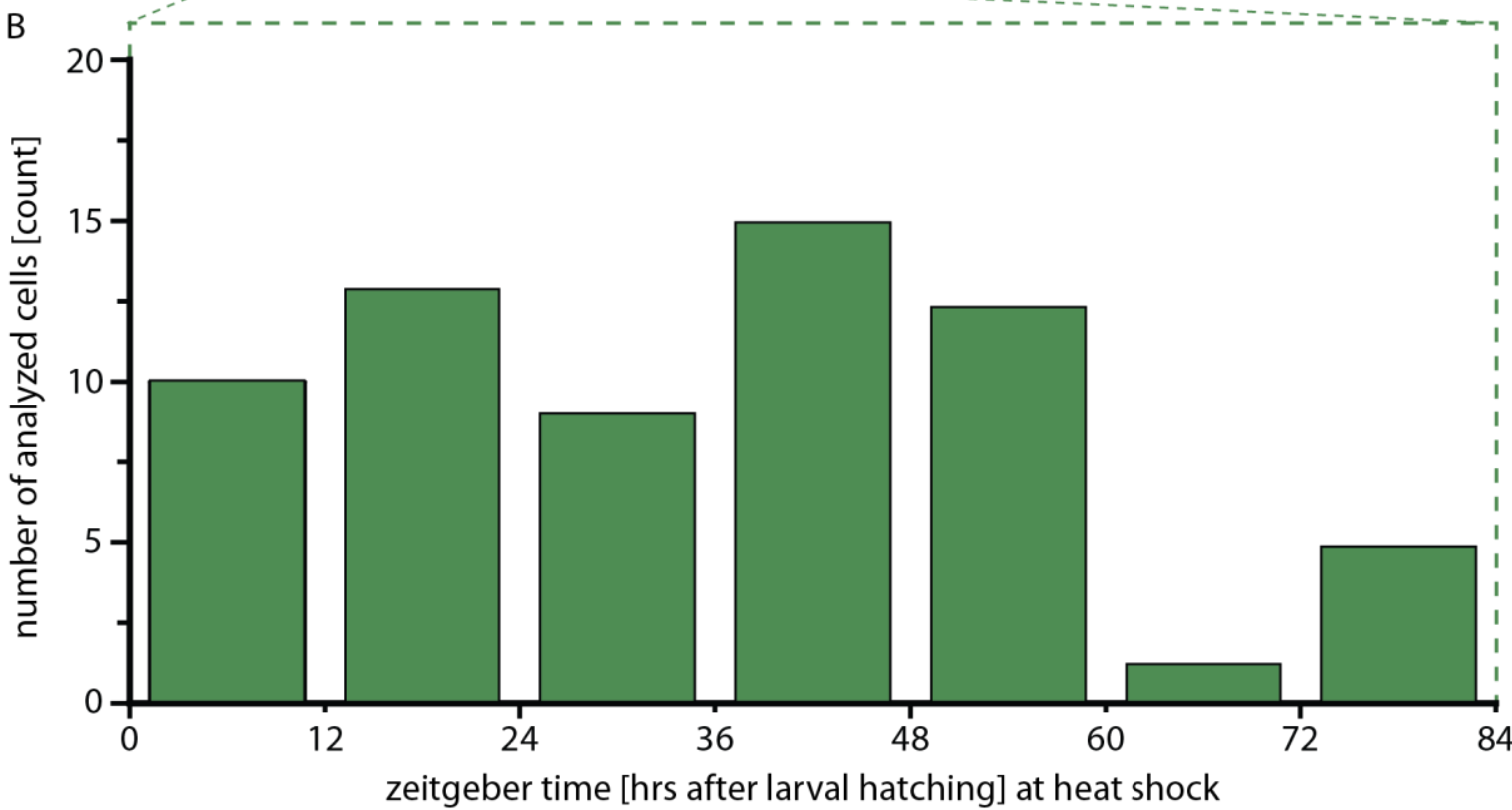

Figure 3.4. Experimental design and time of heat shock of labeled Kenyon cells.

A) Development of the MBs and timeline of the experiment are illustrated over 17 days. In parallel to the timeline of the experiment the developmental status of the mushroom body is illustrated above. Accordingly, the developmental status of the animal is shown below. Color coded text refers to the type of mushroom body Kenyon cells that are generated during that time.

B) 75 single $y$ cell clones were generated using MARCM in combination with 5HT1B-Gal4. It was tried to label $y$ cell clones originating from all time points of $Y$ cell development. Each bar represents the number of $Y$ cells deriving from flies heat shocked at the respective time after larval hatching. It is likely that $y$ cells are born during the time of heat shock, because cell labeling depends on recombination that is induced by heat-shock promoter dependent Flp expression. 
using hexameric mCherry as a reporter and 5HT1B-Gal4 as a driver. Success rates of single clone generation lay around 10-20\%, hence, $\sim 500$ brains were scanned to image 75 single KCs. Examples of multiple KC clone labeling are shown in figure 3.3. In figure $3.4 \mathrm{~A}$, the time course of the experiment is depicted in parallel to the development of the MB lobes. Adult females were used at 4-7 days after imago hatching for experimental analysis (dissection, fixation, confocal imaging). Larvae were age controlled by restricting the egg laying period to a time window of 2-8 hours. Heat shock, to induce Flp expression, was applied in the water bath on $37{ }^{\circ} \mathrm{C}$ for $2-4$
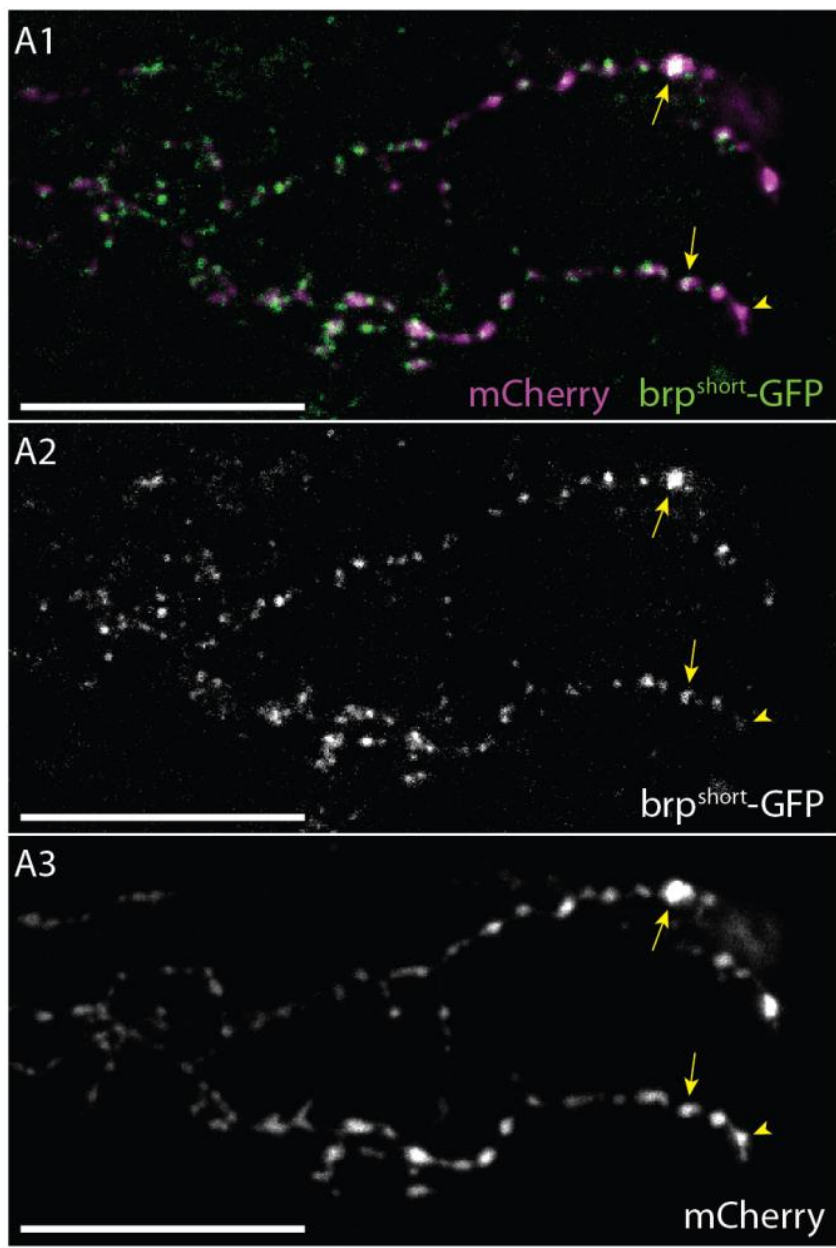

Figure 3.5. Bouton-like structures in $y$ Kenyon cells co-localize with endogenous brp short-GFP expression.

To test if the observed bouton-like structures in the mCherry signal are sites of synaptic contact, the presynaptic active zone marker brp was co-expressed with hexameric mCherry and examined for co-localization.

A1-3) Hexameric mCherry was co-expressed with brpshort-GFP under UAS control in y KCs using the driver line $5 \mathrm{HT} 1 \mathrm{~B}-\mathrm{Gal} 4$. The merge of both channels is shown in $\mathrm{A} 1$, whereas in $\mathrm{A} 2$ the brpshort. GFP signal and in A3 the mCherry signal can be seen. Arrows highlight bouton-like structures of the mCherry signal that nicely co-localize with brpshort-GFP expression. The arrowhead indicates a bouton-like varicosity of the mCherry signal that hardly shows expression of brp ${ }^{\text {short}-G F P}$ and is located towards the end of the cell. Most of the bouton-like structure, however, are located at the same sites as the brp-GFP signal. MARCM dependent expression in a single cell was achieved by heat shocking during development. Scale bar $=30 \mu \mathrm{m}$. 
hrs between 0 and 84 hrs after larval hatching, i.e. during first and second instar stage, the development period of most of the MB y KCs. Birth order might be an important factor determining KC fate and hence morphology. All major classes of KCs are born sequentially (Lee et al., 1999). Therefore, it was tried to generate $\mathrm{Y} \mathrm{KC}$ clones originating from all times of $\mathrm{Y}$ cell development. Though sample sizes were kept similarly, less $y$ cell clones were generated when heat shock was applied more than $60 \mathrm{hrs}$ after larval hatching (figure 3.4B). The reason most likely lies in the fact that MB neuroblasts start switching from $y$ cell to $a^{\prime} \beta^{\prime}$ cell production around that time (Lee et al., 1999). The $~ 75$ embryonic born $y$ dorsal KCs were excluded from this analysis because they have been shown to be involved in visual, not olfactory behavior (Aso et al., 2009, 2014a; Butcher et al., 2012; Kunz et al., 2012; Vogt et al., 2016).

It was noticed, that along the neurites the KCs showed a regular pattern of boutonlike structures (e.g. figure 3.3). In similarity with the larval neuromuscular junctions (Vasin et al., 2014), these bouton-like structures potentially represent areas of increased synaptic occurrence. If indeed, the synapses between KCs and MB output neurons are found here, then the bouton-like structures are supposedly sites of neural plastic changes in olfactory memory formation. An essential protein for the functionality of Drosophila synaptic active zones is Bruchpilot (brp; Wagh et al., 2006).

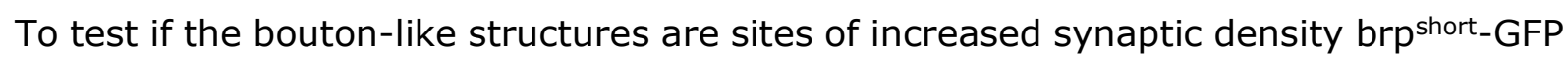
(Fouquet et al., 2009) was co-expressed together with hexameric mCherry in $\mathrm{Y} \mathrm{KC}$ clones (figure 3.5). Confocal images showed a strong accumulation of brpshort-GFP at exactly the regions of the bouton-like varicosities seen in the mCherry signal. However, further physiological and anatomical experiments are required to proof that these bouton-like structures contain functional synapses between KCs and MB output neurons.

\subsubsection{Digital reconstruction of individual Kenyon cells}

In order to describe y KC morphologies in a quantitative manner each cell was digitally reconstructed using the software Neurolucida ${ }^{\circledR}$ (figure 3.6). Reconstruction was performed manually using the simple click tracing function. All parts of the cell were reconstructed and subdivided into cell body, calycal region, peduncle, and lobular region. Dendritic claws within the calyx were counted by setting markers. Quantification included the dendrite length (arborizations in the calyx), the axon length (arborizations in the lobes), as well as the length of the peduncle, the number of dendritic, and axonal nodes and the number of dendritic claws. If the staining quality of the cell was found not to be sufficient for a correct reconstruction it was omitted 

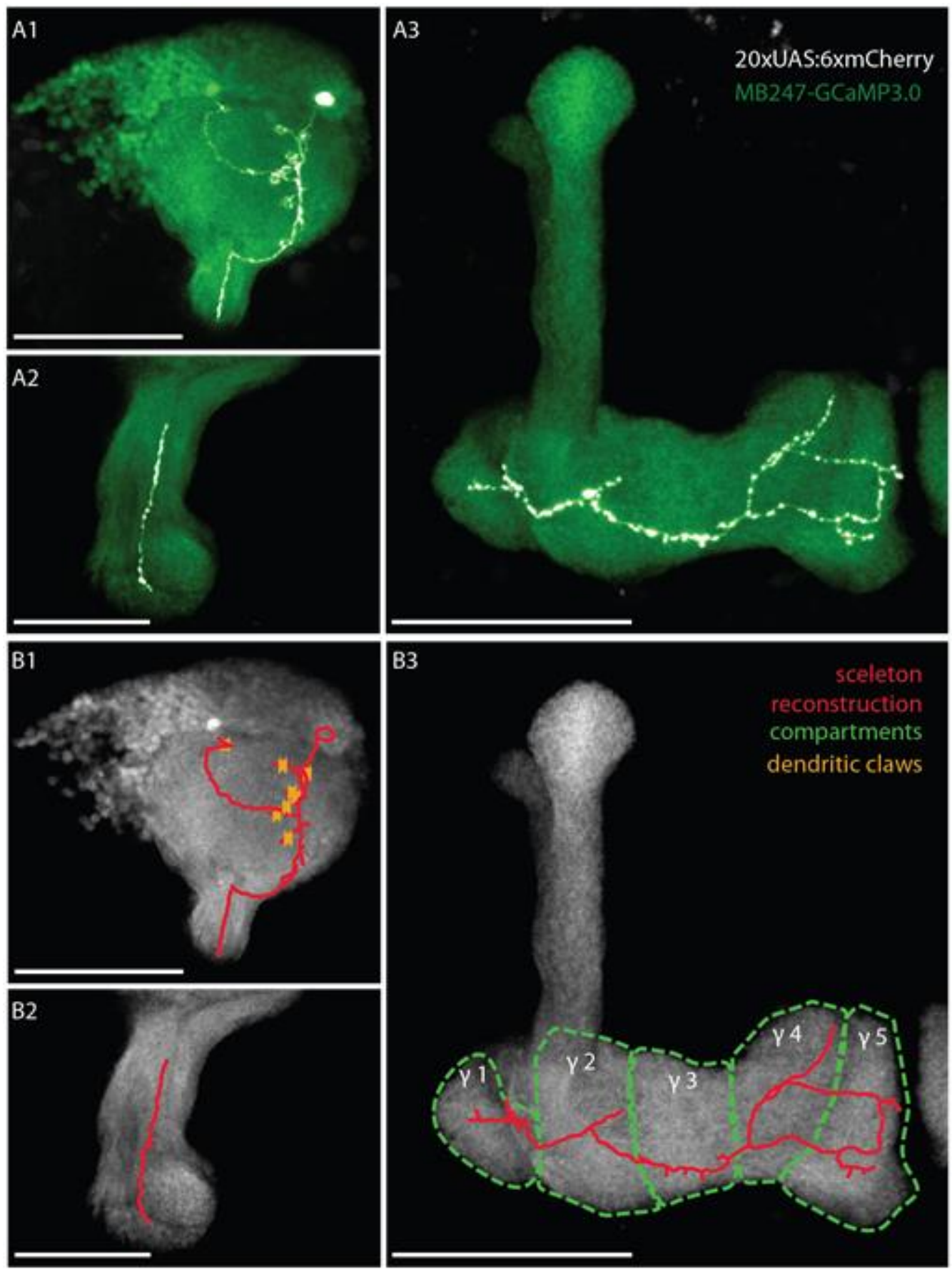

Figure 3.6. Digital reconstruction of $y$ Kenyon cells using Neurolucida.

Quantification of single cell morphologies was achieved by tracing of individual cell shapes using the software Neurolucida $\AA$. Cellular substructures were recognized by inspection of the mushroom body background staining.

A) A single cell MARCM y cell clone is shown within different structures of the MB, the calyx (A1), the peduncle (A2), and the lobes (A3). The $K C$ is labeled by expression of hexameric mCherry, the MB background by expression of GCaMP3.0.

B) The same cell is shown in red as a Neurolucida reconstruction in the calyx (B1), peduncle (B2), and lobes (B3). Yellow crosses mark dendritic claws. The lobes were subdivided into compartments $\gamma 1-\gamma 5$ that were marked by setting green contours which enable morphological quantification within their borders. Scale bar in A1, 3 and B1, $3=50 \mu \mathrm{m}$. Scale bar in A2 and B2 $=30 \mu \mathrm{m}$. 
from the analysis. If only the staining quality in the calyx was insufficient for a correct reconstruction, but staining of axonal arborizations was sufficient to be reconstructed, then these cells were exclusively used in statistical analyses of the MB lobes. The MB background expression of GCaMP was used to compartmentalize KCs into dendrites, peduncle, and axon, as well as to distinguish different MB compartments within the lobes that are innervated by MB extrinsic neurons, like MB output neurons and dopaminergic neurons. The $\mathrm{y}$ lobe compartments $\mathrm{Y} 1-5$ were identified (figure 2.1), labeled, and KC arborizations and the number of nodes within each compartment quantified. Cells that were chosen to be of very good staining quality, i.e. it was possible to reliably identify bouton-like structures, the number of bouton-like varicosities was quantified within $\mathrm{y} 1$. In sum, the quantified parameters represent all parts of KC morphology and shall be used for description of the $\mathrm{Y}$ main $\mathrm{KC}$ population based on individual KCs.

\subsection{Anatomical characterization of $\mathrm{y}$-type Kenyon cells}

Two approaches were taken for the characterization of the measured $\mathrm{Y}$ main $\mathrm{KC}$ population. First, using only parameters that apply to the overall Kenyon cell morphology, i.e. dendrite length, number of dendritic claws, number of axonal nodes, and axon length. Second, using parameters that apply specifically to the lobular arborizations, i.e. the length of arborizations within $\mathrm{Y} 1-5$, because the memory trace is believed to be localized within the lobes, at the synapses between KCs and MB output neurons that are modulated by reinforcement signals (Fiala, 2007; Heisenberg, 2003; Owald and Waddell, 2015).

\subsubsection{Quantitative description of $\mathbf{y}$ cell morphology}

The main subdivisions of a KC are the dendritic arbors in the calyx, where olfactory input is conveyed from PNs to KC dendritic claws, and the axonal arbors in the lobes, where KCs synapse onto MBONs (Fahrbach, 2006). If the morphological identity of a neuron is interconnected with its functionality, then the morphological analysis of KCs input and output pathways should contribute to their classification (Connors and Regehr, 1996). Several KC subtypes have been identified mainly based on their gene expression patterns (e.g. Aso et al., 2009). Interestingly, all described subtypes have unique axonal projection profiles and can be characterized solely by their lobular arborizations (Aso et al., 2014a; Tanaka et al., 2008). Although less defined, dendritic arbors of described KC subtypes also innervate discrete zones within the MB calyx (Lin et al., 2007). PNs have successfully been classified using data about their axonal arborizations in the calyx, which correlated to the respective dendritic innervations of 
distinct glomeruli of the antennal lobes (Jefferis et al., 2007; Wong et al., 2002). Among the four parameters that were sufficient to define PN calycal arborizations were the number of calycal boutons, and the length of axonal arborizations (Wong et al., 2002). In the present study, two calycal and two axonal parameters were selected for the detailed analysis of gross $y$ cell morphology. For the calyx, the number of dendritic claws, and the length of dendritic arborizations, for the lobes, the length of axonal arborizations, and the number of axonal nodes. The distributions of all four parameters are shown as dot plots in figures 3.7-3.10 together with selected confocal images that were chosen in order to represent the whole characteristic range of the respective parameter. An overview of the distribution of all the quantified parameters is given in table 3.1.

KC dendritic claws are specialized dendritic arborizations recognized by their characteristic shape (Butcher et al., 2012; Leiss et al., 2009; Strausfeld et al., 2003; Yasuyama et al., 2002). They receive olfactory input from PN boutons (Fiala et al., 2002; Gruntman and Turner, 2013). Claws from one KC form contacts to multiple boutons, while each claw contacts normally a single PN bouton, though complex claws that contact more than one PN bouton have been described (Gruntman and Turner, 2013; and inset in figure 3.7B). It was suggested, to separate claws into simple (consisting of 2 branches) and complex (consisting of more than two branches;
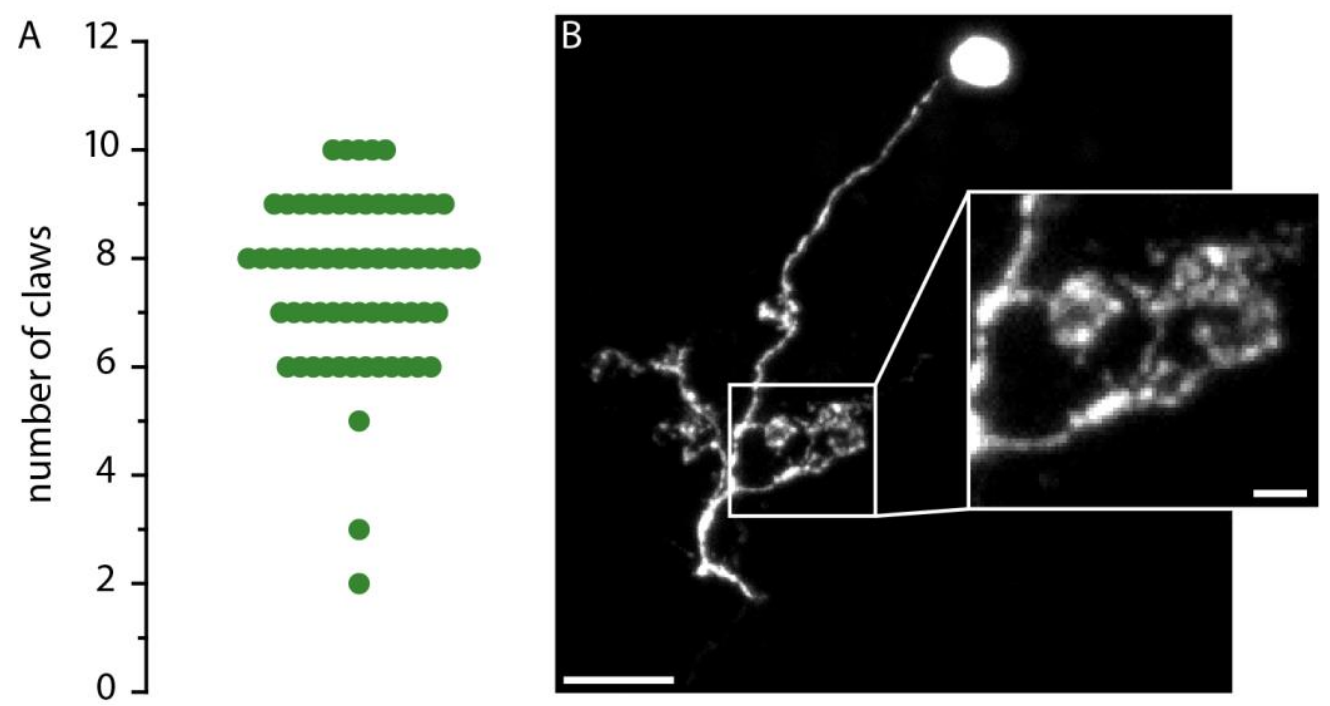

Figure 3.7. Quantification of the number of y Kenyon cells' dendritic claws.

Dendritic claws are specialized Kenyon cell input structures. They are formed within the calyx, and usually one claw is connected to one presynaptic projection neuron bouton. The more claws respond to a given odor stimulus, the more likely the Kenyon cells shows a spiking response.

A) The number of dendritic claws in the analyzed $\mathrm{Y} \mathrm{KC}$ population is shown as a dot plot. $1^{\text {st }}$ quartile $=7$, median $=8,3^{\text {rd }}$ quartile $=9$ claws, average number of claws $=7.58, \mathrm{~N}=65$.

B) Example image of the arborizations of a $Y$ KC in the MB calyx. Magnification shows an area of increased claw occurrence. Scale bar $=10 \mu \mathrm{m}$, inset $=2 \mu \mathrm{m}$. 
Butcher et al., 2012). This distinction, however, could not be observed in the present study.

The entity of one PN bouton and its synaptic partners constitute a microglomerulus. It has been estimated that the Drosophila MB calyx consists of $\sim 1,000$ microglomeruli, hence 1,000 PN presynaptic boutons, which are opposed by $\sim 12,500 \mathrm{KC}$ dendritic claws; according to this, there are 12.5 claws per PN bouton (Butcher et al., 2012; Leiss et al., 2009). Interestingly, y KCs are believed to contribute to 70-90\% of all calycal microglomeruli (Leiss et al., 2009). The average number of dendritic claws of one y KC was measured to lie around 7.3 (Butcher et al., 2012). In the present study, the number was found to be slightly higher, with an average around 7.6 and a median number of 8 claws per $\mathrm{Y} K \mathrm{KC}$ (figure 3.7A and table3.1). The difference might be explained by a better optical resolution, and thus more successful claw identification, achieved by using a confocal Leica SP8 equipped with hybrid detectors against a Leica SP2 and photomultipliers. The range of dendritic claws was found in the present study to be between 2 and 10 claws per KC, though higher numbers of up to 12 claws per $\mathrm{Y}$ KC have been reported (Butcher et al., 2012).

As a second calycal variable, the length of dendritic arborizations was measured. In comparison to the dendritic claws that reflect olfactory input to KCs from presynaptic PNs (Gruntman and Turner, 2013), the dendrite length is a measure that reflects the general size of the cell, and the area it occupies within the calyx. Moreover, the cell's size might also reflect the cell's electrical activity. A model of the firing properties of neocortical neurons, could explain differences in cellular spiking by solely different dendritic geometries (Mainen and Sejnowski, 1996).

Table 3.1. Descriptive statistical analysis of $y$ Kenyon cell morphological parameters

\begin{tabular}{|c|c|c|c|c|c|c|c|c|}
\hline & $\min$ & median & $\max$ & mean & SEM & $\mathrm{N}$ & $\begin{array}{l}\text { test on } \\
\text { normality*[p- } \\
\text { value] }\end{array}$ & figure \\
\hline $\begin{array}{l}\text { dendrite } \\
\text { length }[\mu \mathrm{m}]\end{array}$ & 92.6 & 226.8 & 338.8 & 223.65 & 5.95 & 65 & 1.0 & 3.8 \\
\hline $\begin{array}{l}\text { dendrite } \\
\text { nodes } \\
\text { [count] }\end{array}$ & 5 & 15 & 30 & 15.40 & 0.60 & 65 & 0.333 & $\begin{array}{c}\text { not } \\
\text { shown }\end{array}$ \\
\hline $\begin{array}{l}\text { dendritic } \\
\text { claws } \\
\text { [count] }\end{array}$ & 2 & 8 & 10 & 7.58 & 0.19 & 65 & 0.0004 & 3.7 \\
\hline $\begin{array}{l}\text { peduncle } \\
\text { length }[\mu \mathrm{m}]\end{array}$ & 56.7 & 80.7 & 169.1 & 82.70 & 1.79 & 73 & $<0.001$ & $\begin{array}{c}\text { not } \\
\text { shown }\end{array}$ \\
\hline $\begin{array}{l}\text { axon length } \\
{[\mu \mathrm{m}]}\end{array}$ & 129.9 & 294.6 & 407.2 & 292.75 & 6.62 & 65 & 1.0 & 3.9 \\
\hline $\begin{array}{l}\text { axon nodes } \\
\text { [count] }\end{array}$ & 5 & 12 & 28 & 13.78 & 0.61 & 65 & 0.061 & 3.10 \\
\hline
\end{tabular}


Here, the dendrite length was defined as the length of the neurite between the cell body and the exit point of the calyx, which was identified via visual inspection of the MB background staining. Except for $\gamma$ dorsal and $a / \beta$ posterior KCs no KCs build dendritic claws outside the main calyx (Aso et al., 2014a). In this study, a vast range of magnitudes of dendritic arborizations was observed (figure 3.8, and table 3.1). The shortest $\mathrm{KC}$ found, has a dendrite length of $92.6 \mu \mathrm{m}$, while the longest $\mathrm{KC}$ has a

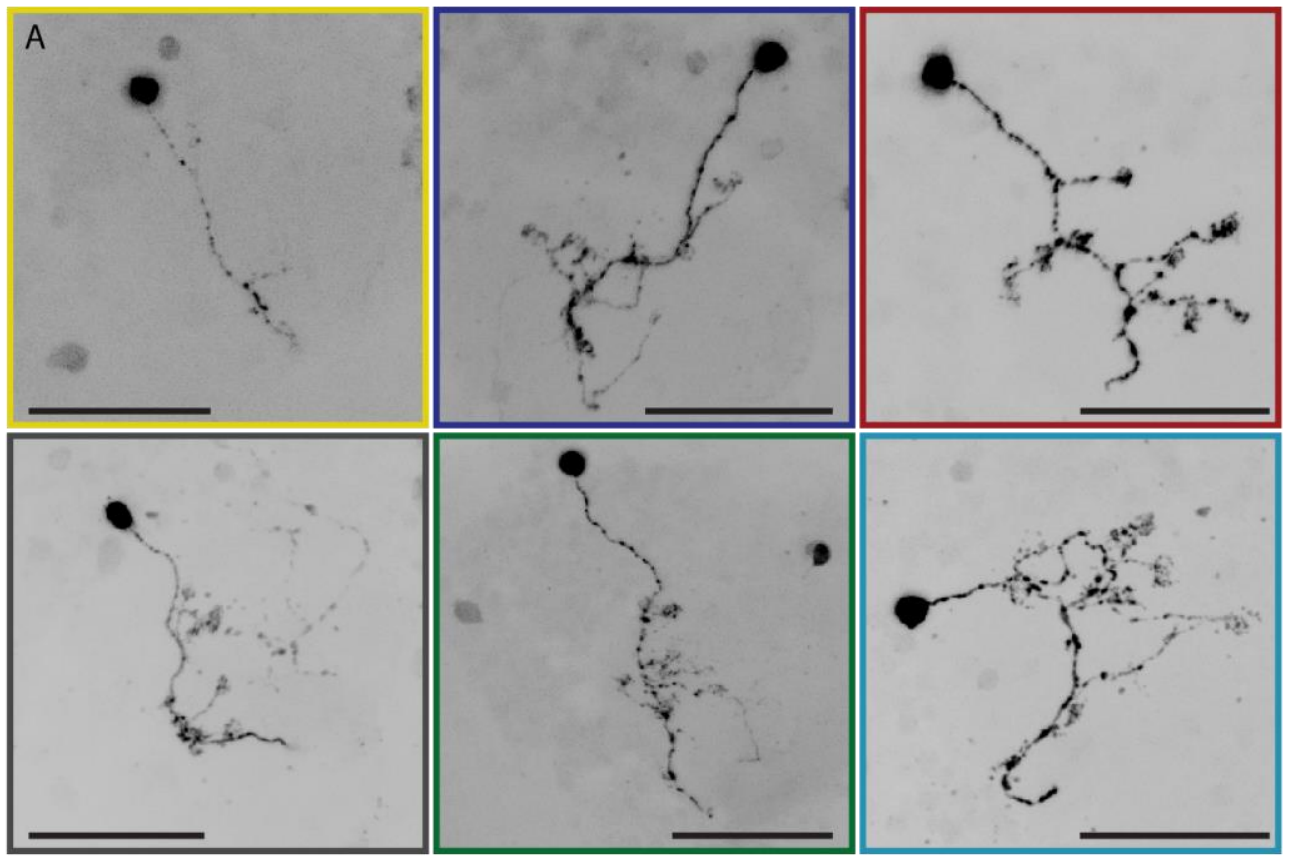

B
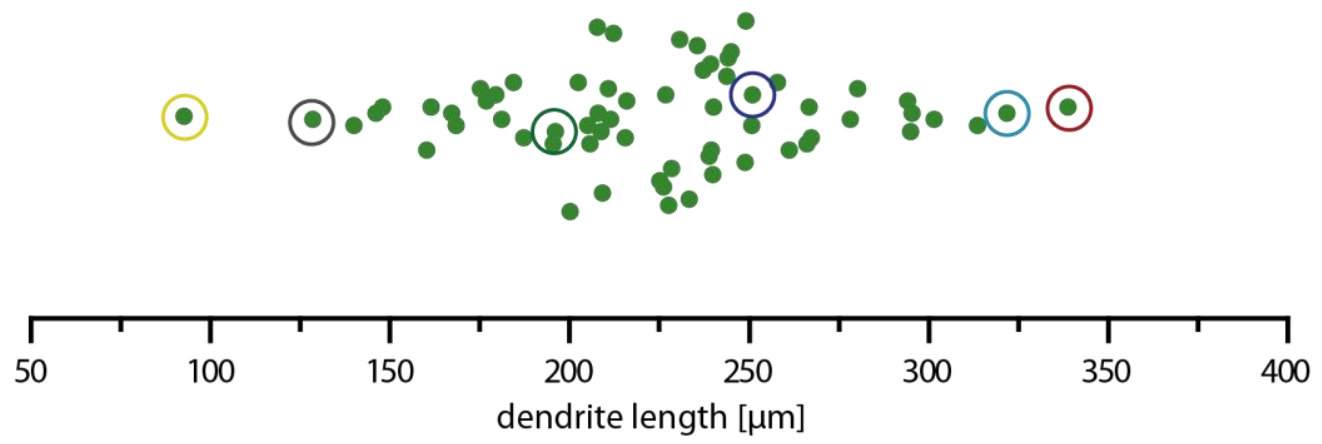

Figure 3.8. Quantification of y Kenyon cells' dendrite length.

Although in insect unipolar neurons a differentiation between dendrites and axons is not trivial, it is widely accepted that Kenyon cell calycal arborizations are dendritic. The dendrite length was quantified as one of the main parameters describing Kenyon cell morphology.

A) Example images of the dendrites of $\mathrm{Y} K C s$ of various sizes covering the whole range of magnitudes. Image frames are color coded according to the KCs highlighted by circles in B. Scale bars $=30 \mu \mathrm{m}$.

B) The length of the dendrites in the analyzed y KC population is shown as a dot plot. Circles indicate example KCs shown in A. $1^{\text {st }}$ quartile $=196.1 \mu \mathrm{m}$, median $=226.8 \mu \mathrm{m}$, $3^{\text {rd }}$ quartile $=$ $249 \mu \mathrm{m}$, average length $=223.6 \mu \mathrm{m}, \mathrm{N}=65$. 
dendrite length of $338.8 \mu \mathrm{m}$. The median dendrite length is at $226.8 \mu \mathrm{m}$ with $52.9 \mu \mathrm{m}$ interquartile range. It was observed that KCs usually build a main neurite trunk, from which thinner arbors branch off. At the end of such a thinner branch often one or several dendritic claws are located. Although various sizes of side branches were found their lengths' were not quantified.

The lobes are the most prominent structures of the MBs due to their characteristic split into the vertical $a$ and $a^{\prime}$ lobes and the horizontal $\beta, \beta^{\prime}$, and y lobes (Crittenden et al., 1998). Here, only y KCs were analyzed that enter the $y$ lobe laterally via the heel, also called $\mathrm{Y} 1$, and extend medially into compartments $\mathrm{Y} 2-5$. An important feature of KCs is the connectivity to extrinsic neurons. It has been shown that $\mathrm{Y}$ KCs are closely connected to aminergic neurons, including serotoninergic, octopaminergic, and dopaminergic ones (Pech et al., 2013b), and were reported to activate subsequent MB output neurons (Barnstedt et al., 2016; Hige et al., 2015b). Bouton-like structures are distributed regularly along axonal processes of $\mathrm{Y}$ KCs (see figure 3.5), however,
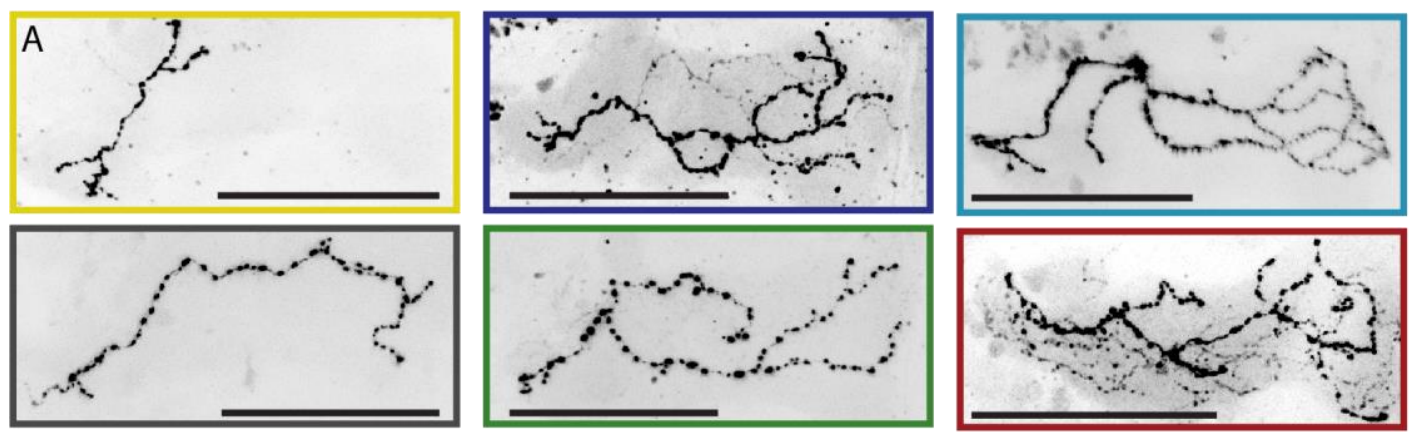

B

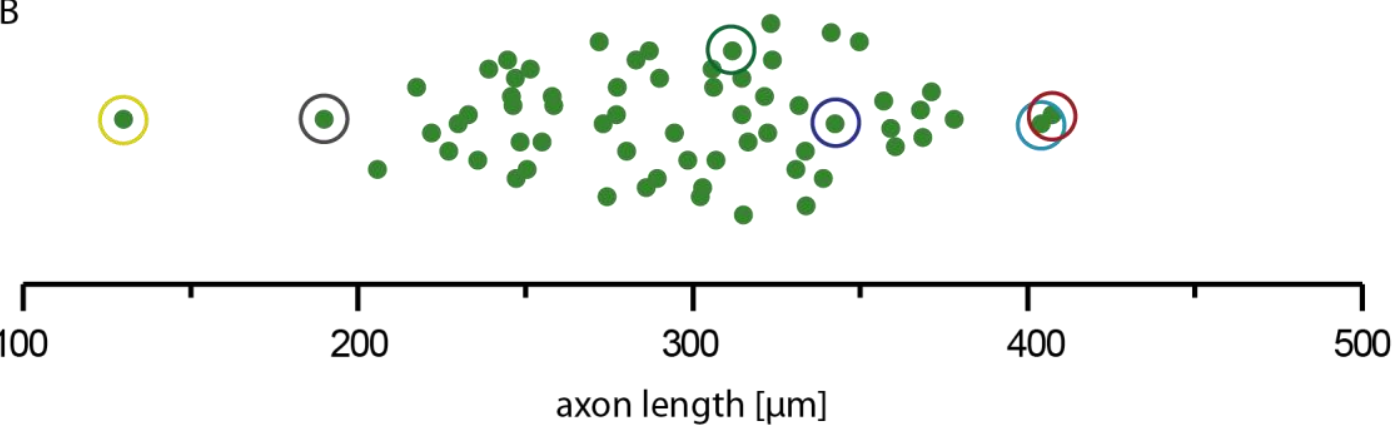

Figure 3.9. Quantification of $y$ Kenyon cells' axonal length.

y Kenyon cell axons are here considered to be the arborizations within the $y$ lobe, where Kenyon cells form synaptic connections with extrinsic dopaminergic and mushroom body output neurons. A) Example images of the axons of $\mathrm{Y} K \mathrm{KCs}$ of various sizes covering the whole range of magnitudes. Image frames are color coded according to the $\mathrm{KCs}$ highlighted by circles in B. Scale bars $=30$ $\mu \mathrm{m}$.

B) The axon length in the analyzed Y KC population is shown as a dot plot. Circles indicate example KCs shown in A. $1^{\text {st }}$ quartile $=250.5 \mu \mathrm{m}$, median $=294.6 \mu \mathrm{m}, 3^{\text {rd }}$ quartile $=330.8 \mu \mathrm{m}$, average length $=292.7 \mu \mathrm{m}, \mathrm{N}=65$. 
counting of the number of boutons was not possible in most of the KCs of this study because of insufficient staining quality. Thus, the lengths of axonal arborizations were quantified as an approximation for connectivity. The length was measured from the KCs entry point into the heel, and included all subsequent branches within the $y$ lobes. Identification of the heel region was achieved by visual inspection of the MB background staining. As for the dendritic arbors, the range of lengths of axonal arbors was broader than expected (figure 3.9 and table 3.1). Usually, all Y lobe KCs extended their arbors until the $\mathrm{y} 5$ compartment. However, one cell was found that only extends until $y 2$. This was the shortest cell with an axonal length of $129.9 \mu \mathrm{m}$. The second shortest cell, which has arborizations until $ү 5$, was found to be $189.8 \mu \mathrm{m}$ long. The cell with the longest axonal arbors has a magnitude of $407.2 \mu \mathrm{m}$. The median length of the axonal arborizations is at $294.6 \mu \mathrm{m}$ with $80.3 \mu \mathrm{m}$ interquartile range.

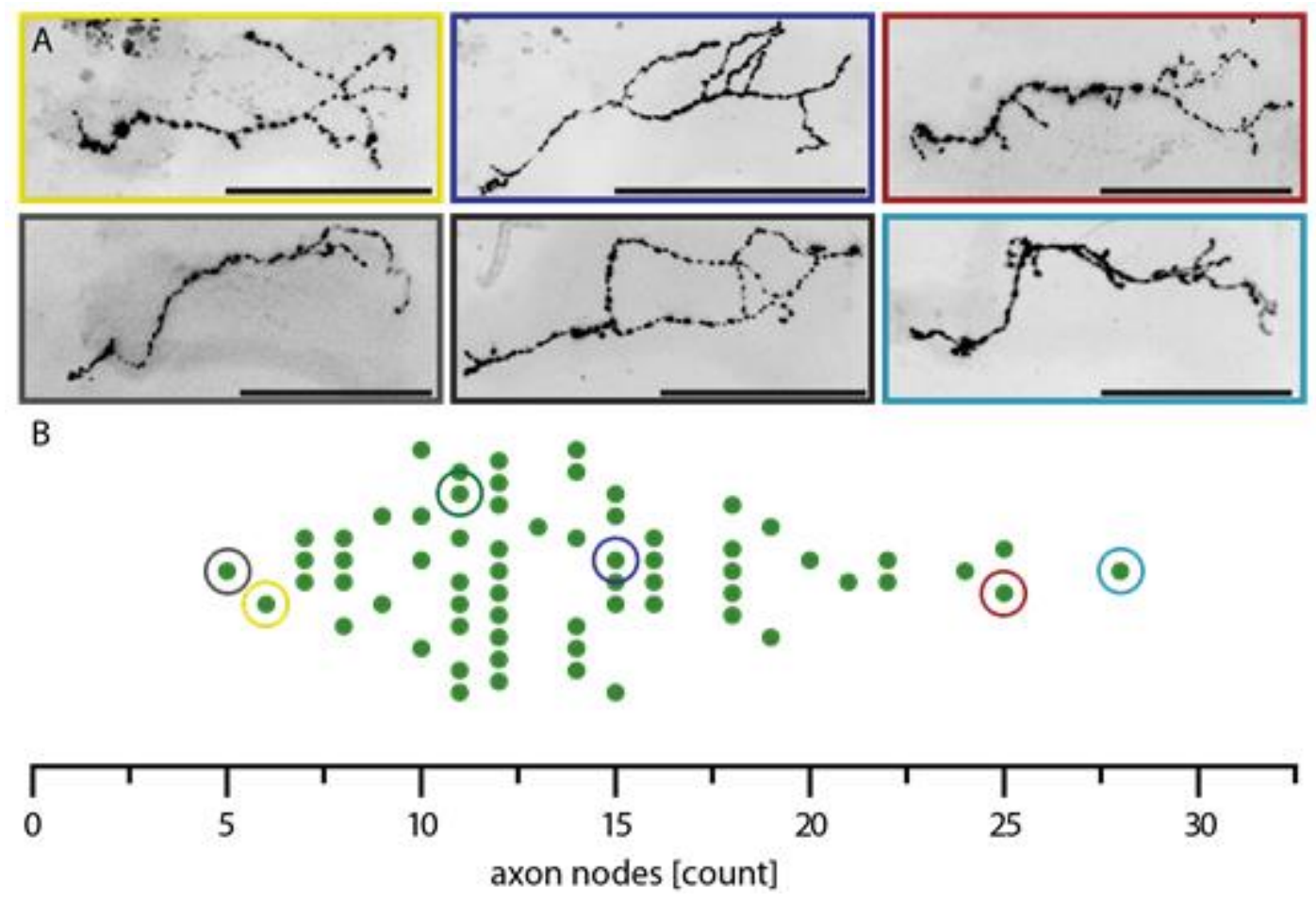

Figure 3.10. Quantification of the number of $y$ Kenyon cells' axonal nodes.

Each point of bifurcation, here, within the lobe structure, is considered to be an axon node. The number of axon nodes can be considered to be a measure of the axons arborizations complexity. A) Example images of axons of $\mathrm{Y} \mathrm{KCs}$ with a various number of nodes covering the whole range of magnitudes. Image frames are color coded according to the KCs highlighted by circles in $\mathrm{B}$. Scale bars $=30 \mu \mathrm{m}$.

B) The number of axon nodes in the analyzed $y$ KC population is shown as a dot plot. Circles indicate example KCs shown in $\mathrm{A} .1^{\text {st }}$ quartile $=11$, median $=12,3^{\text {rd }}$ quartile $=16$ nodes, average number of nodes $=13.8, \mathrm{~N}=65$. 
As a second axonal parameter, the number of axonal nodes were taken, as a measure of complexity of axonal geometry. In a recent study overexpression of the scaffold protein DISC1, that has been associated with mental disorders in humans, was found to suppress axonal length and the number of axon nodes of $\mathrm{Y}$ KCs (Furukubo-Tokunaga et al., 2016). Furthermore, the number of axonal nodes in Y KCs was reduced after ChR2 induced activation of KCs (Tessier and Broadie, 2008). Quantification of the number of axonal nodes in control animals revealed numbers between $\sim 5$ (FurukuboTokunaga et al., 2016) and 10 (Tessier and Broadie, 2008). In the present study, the total count of nodes within the $y$ lobes were found to be even higher, with a median number of 12 (interquartile range of 5 ), ranging from 5 to 28 nodes per cell.

A closer look at the distributions of the four parameters, i.e. the claw number, the dendrite length, the axon length, and the number of axon nodes, can help to identify novel Y KC subtypes. Irregular patterns within the distributions can be a hint for

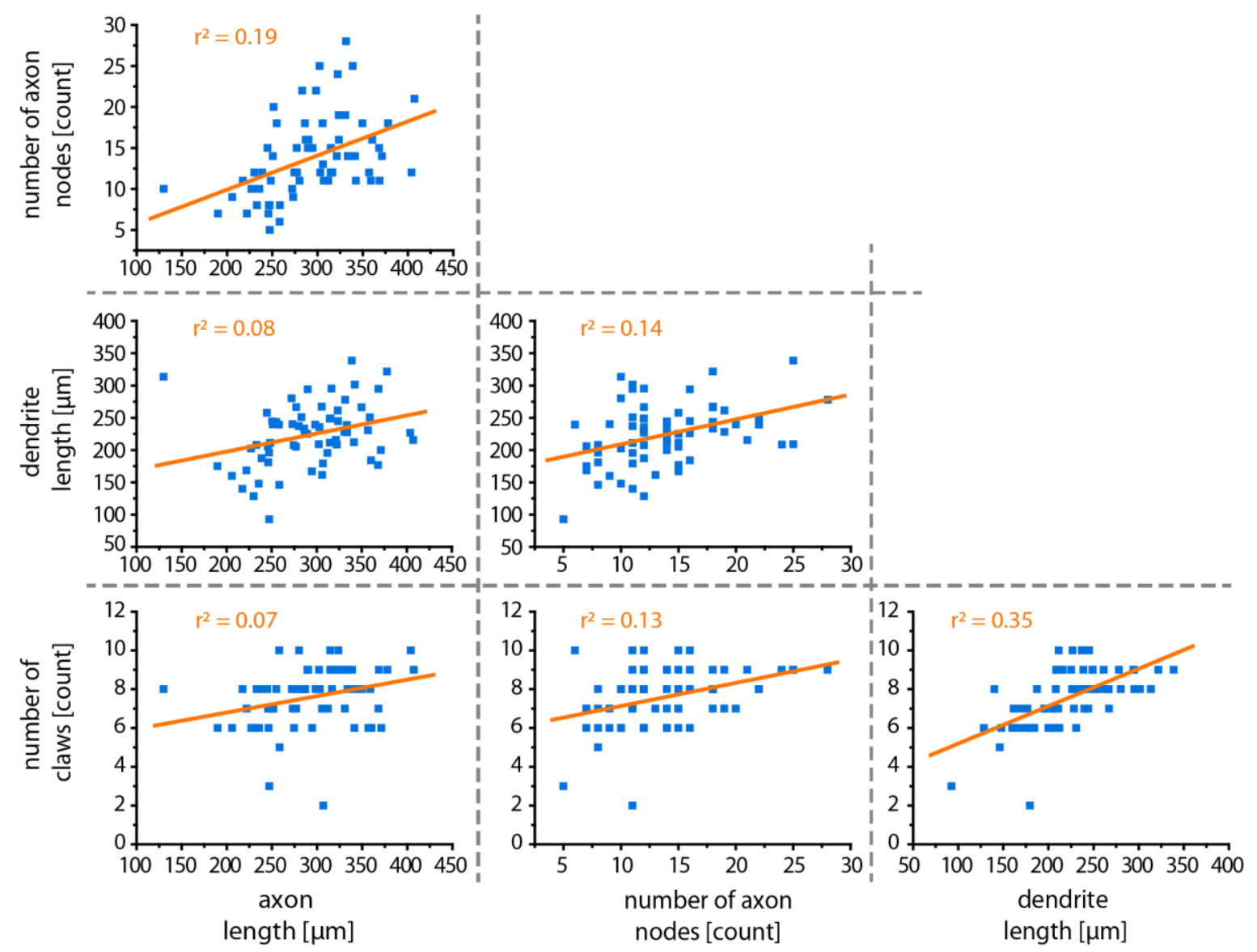

Figure 3.11. Correlations between parameters describing y Kenyon cell morphology.

The matrix plot shows the pairwise correlations among all four parameters described in figure 3.7-3.10. i.e. the number of dendritic claws, the length of axonal and calycal arborizations, and the number of axon nodes. The correlation between any pair of parameters has been estimated using a simple linear regression model implemented in Origin 8.5. All regression models significantly fit the data. The percentage of variance in the data covered by the linear model is given as the $r^{2}$ value that lies between 0 and 1 . 
distinct $\mathrm{Y}$ KC types that are specifically different in the development of certain cell characteristics. Although, for each of the four parameters different $\mathrm{Y}$ KCs can adopt a wide range of values, there is no clear indication for a subdivision. When tested with the Shapiro-Wilk test, the hypothesis of normal distribution for the distributions of the peduncle lengths and the number of dendritic claws had to be rejected (see table 3.1). Differences in peduncle length likely arise from differences in the size of the brains, which was not controlled in this study. To substantiate the idea of additional $\mathrm{Y} \mathrm{KC}$ subdivisions due to, for example, differences in the number of claws, has to be shown by further analyses.

In a next approach, the four parameters, dendritic claws, dendrite length, axon length, and axon nodes, were tested for dependencies among each other. Linear regression was employed to analyze pairwise correlations. It was found that linear models appropriately fit the data. However, the analyses revealed only weak correlations, the strongest being between the number of dendritic claws and the dendrite length (figure 3.11). Since dendritic claws also contribute to the overall length of the dendrites a correlation between the two parameters can be expected. Thus, there is a general trend of KC morphology, in the sense that the larger is one part of the cell, the larger are the other parts too. However, only a small fraction of the variance was covered by linear regression.

\subsubsection{Descriptive statistical analysis of $\mathrm{y}$ Kenyon cells' morphology}

\subsubsection{Subdivision of $y$ Kenyon cells based on bifurcation}

Quantitative data analysis could demonstrate that $\mathrm{Y} K \mathrm{KC}$ are highly versatile in their morphology in regard to four parameters that were chosen to describe gross $y$ cell appearance (figures 3.7-3.10). In a next step, qualitative analysis was employed to classify the stained set of 75 single $y$ KCs. All classes of $a^{\prime} / \beta^{\prime}$ and $a / \beta$ KCs bifurcate at the base of the $a^{\prime} 1$ or a1 compartment to form the vertical $a^{\prime}$ and a lobes and the horizontal $\beta^{\prime}$ and $\beta$ lobes respectively (Crittenden et al., 1998). Likewise, Y KCs before being remodeled during pupal stage, bifurcate in the larval MBs to innervate both the vertical and the medial lobe (Technau and Heisenberg, 1982). The adult $Y$ KCs are known to branch only within the horizontal lobes (Aso et al., 2014a). Here, it was noticed that $y$ cell axons often build two major branches of similar magnitude, that appear quite similar to the bifurcation seen in the other cell types. In order to define a bifurcation the primary node of each $\mathrm{y} K \mathrm{KC}$ was determined. A node was counted a primary node if both subsequent branches crossed at least two compartment borders or, in the case of a node in the $\mathrm{\gamma} 4$ compartment, if both branches were of similar 


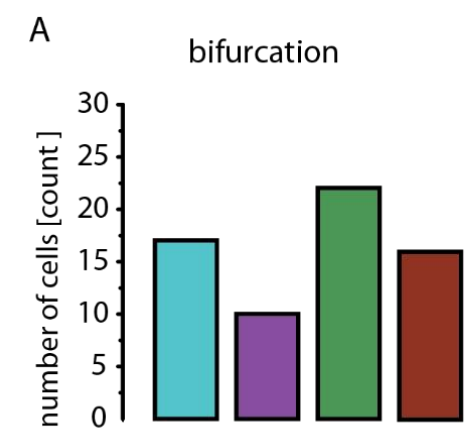

B

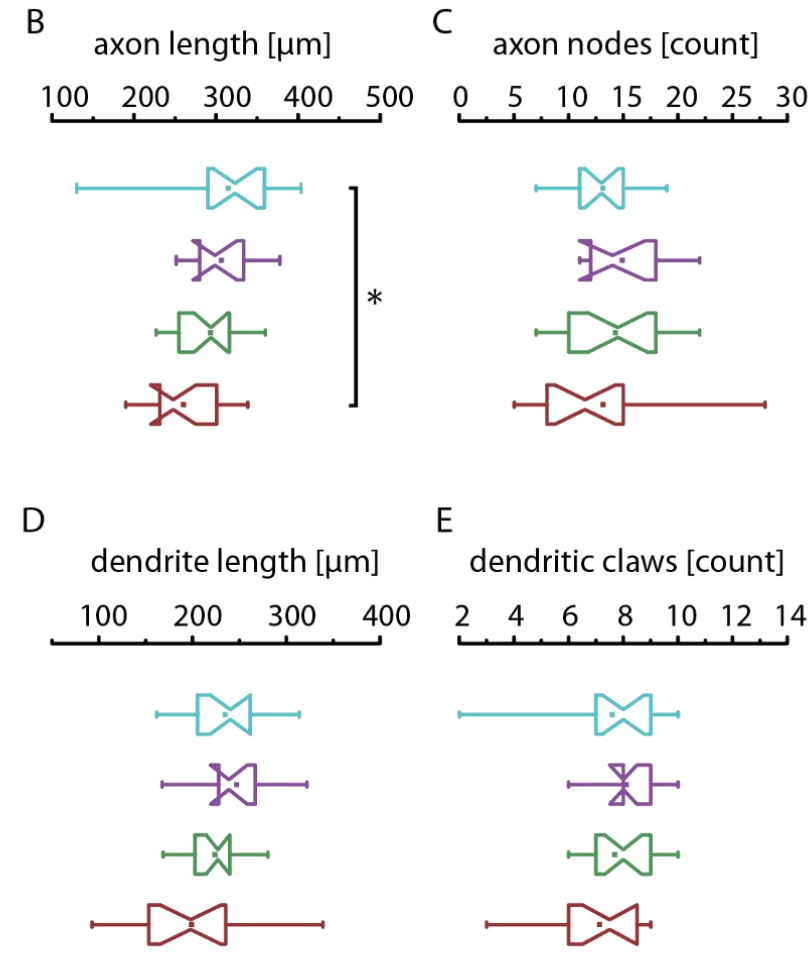

C axon nodes [count]

$\mathrm{D}$

primary node in $\mathrm{\gamma} 2$

primary node in $\gamma^{3}$

primary node in $\gamma 4$

not bifurcating
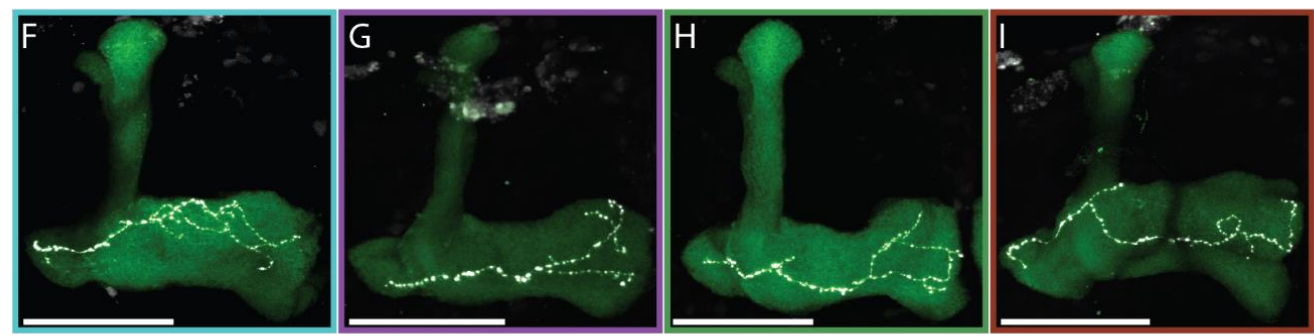

Figure 3.12. Subdivision of $\mathbf{y}$ Kenyon cells by point of bifurcation.

In contrast to $a^{\prime} / \beta^{\prime}$ and $a / \beta$ KCs $\gamma$ KCs do not bifurcate to extend one branch in the vertical and another branch in the horizontal lobes. However, $\mathrm{Y}$ KCs often split within the $\mathrm{Y}$ lobes to extend two branches that run in parallel till the end of the lobe. The criteria to determine the primary node that constitutes a bifurcation of the cell were that both subsequent branches cross at least two compartment borders or in the case of a primary node in $\mathrm{Y} 4$ build two branches that extend into $\mathrm{y} 5$ and are of similar magnitude.

A) The number of cells belonging to each of the four groups: primary node in $\mathrm{y} 2,3$, or 4 , and not bifurcating. KCs with a primary node in $\mathrm{Y} 1$ were not found.

B) Comparison of the axon lengths between the four bifurcation groups, which are indicated by the colors used in A. Significance was found only between the non bifurcating and the group with the primary node in $\mathrm{y} 2$.

C) Comparison of the number of axon nodes between the four bifurcation-groups, which are indicated by the colors used in A. No significant difference was found.

D) Comparison of the dendrite lengths between the four bifurcation-groups, which are indicated by the colors used in A. No significant difference was found.

E) Comparison of the number of dendritic claws between the four bifurcation-groups, which are indicated by the colors used in A. No significant difference was found.

Boxes indicate $1^{\text {st }}$ quartile, median, and $3^{\text {rd }}$ quartile, notches the $95 \%$ confidence interval. The small rectangle within the box represents the mean. Whiskers indicate 5 and 95 percentiles.

* indicates significance $\mathrm{p}<0.05$, \# test

$\mathrm{F}-\mathrm{I})$ Example $\mathrm{KC}$ images representing each of the respective bifurcation groups, which are indicated by color coded image frames that fit the colors used in A. Scale bars $=30 \mu \mathrm{m}$. 
magnitude, and both crossed the border to the $\gamma 5$ compartment. According to the location of the primary node the cells were divided into four groups. One group with cells that are not bifurcating, and three groups with a primary node in $\mathrm{y} 2, \mathrm{Y} 3$, or $\mathrm{y} 4$. Future research has to show, if the observed bifurcations serve a functional purpose, or if there are specific three dimensional innervation patterns of bifurcating cells as has been found for the $a^{\prime} / \beta^{\prime}$ anterior-posterior versus the $a^{\prime} / \beta^{\prime}$ middle neurons (Aso et al., 2014a).

To test if the observed anatomical subdivision of $\mathrm{Y}$ KCs based on bifurcation is related to the basic parameters used to describe gross Y KC morphology, the four 'bifurcation' groups were compared statistically in respect to the claw number, the dendrite length, the axon length, and the number of axon nodes (figure 3.12). A significant difference between groups was found in the axon length between the non-bifurcating group and the group with a primary node in $\mathrm{y} 2$. This difference, however, is likely due to the nature of the bifurcation, which contributes to the length of KC lobular arborizations. Comparisons to the other groups as well as comparisons of the other parameters revealed no differences. Thus, there is no clear relationship between within $y$ lobe bifurcation and parameters describing gross $\mathrm{y}$ KC morphology.

\subsubsection{Subdivision of $y$ Kenyon cells by hierarchical clustering}

Unsupervised clustering algorithms have been used in in a large variety of fields in order to identify elements of a group that are distinguished from other elements of the group by similarity (Jain et al., 1999). Here, hierarchical agglomerative clustering was applied to the set of $\mathrm{Y}$ lobe KCs described by gross morphological parameters. Dissimilarities between data points were calculated as Euclidean distances in a 4dimensional space. Equal contribution of each parameter to the clustering was ensured by scaling of all entries of a respective parameter to its standard deviation. Agglomerative clustering is a 'bottom-up' approach; starting from each data point as one single cluster, clusters are merged into larger ones to finally build one single cluster. The resultant hierarchical distance tree is typically depicted as a dendrogram. As a criterion to merge pairs of clusters Ward's method (also known as Ward's minimum variance method) was chosen, which is based on the minimization of the within-cluster sum of squares (Ward, 1963). Ward's method has been proven in several test studies to be more successful in retrieving original clusters of natural data sets in comparison to other commonly used methods (Blashfield, 1976; Hands and Everitt, 1987). In order to analyze neuromorphology and neurophysiology, Ward's method has been applied to distinguish for example neocortical interneurons (Cauli et 

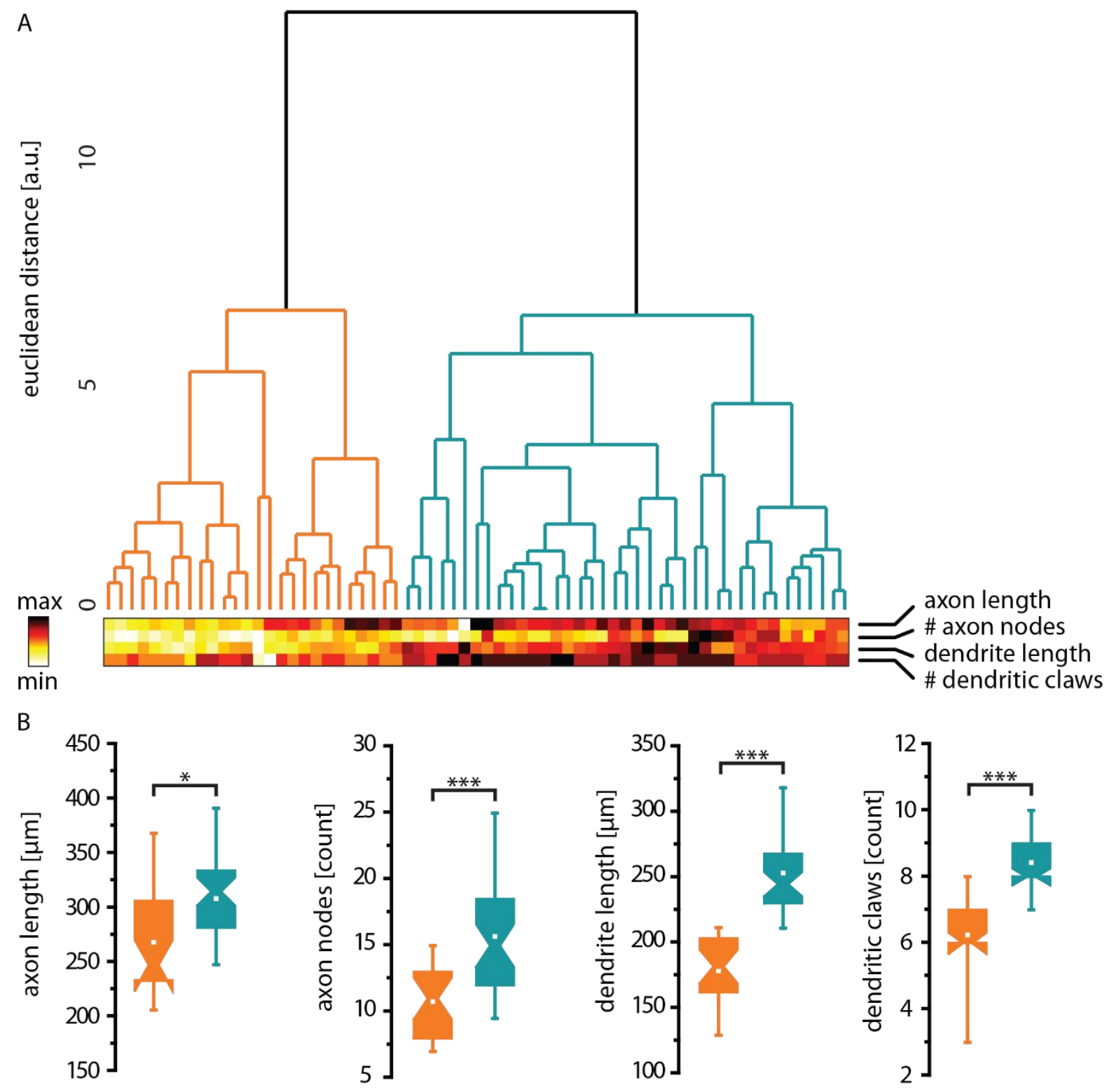

C
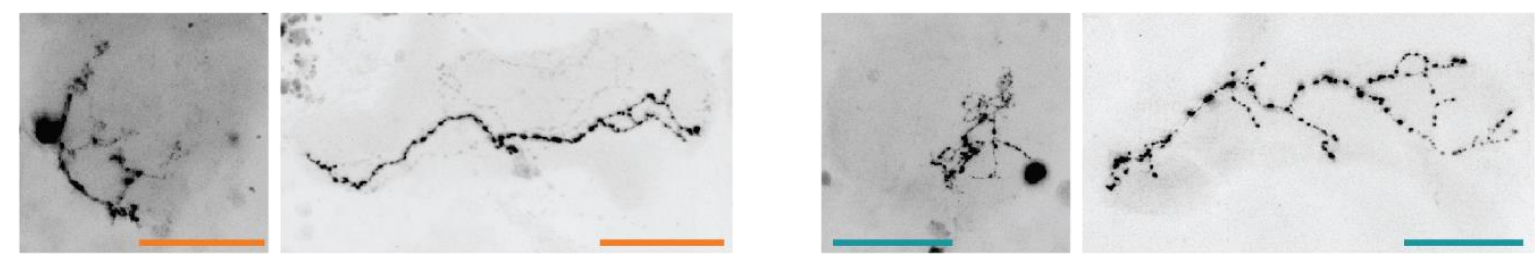

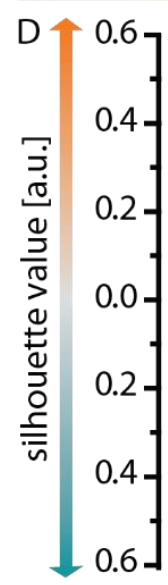

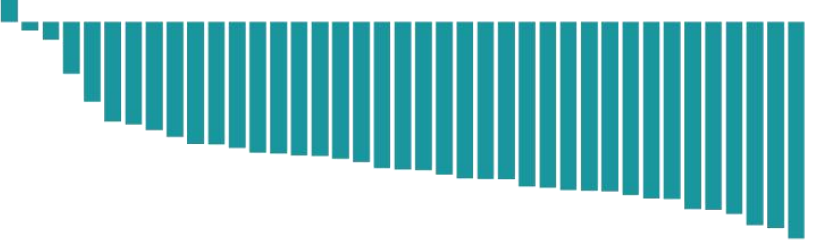


al., 2000; Karagiannis et al., 2009), and Drosophila olfactory PNs (Jefferis et al., 2007; Wong et al., 2002).

In the present analysis, y KCs were grouped into two 'main' clusters (figure 3.13A), and four 'sub' clusters (figure 3.14A). The two 'main' clusters contain 36 and 29 cells respectively that are significantly different in all four parameters (figure 3.13B). The larger of the two clusters in terms of cell number has more dendritic claws, longer dendrites, and axons, and more axonal nodes than the smaller cluster. Thus, there is a general subdivision of $\mathrm{y}$ KCs into large and small cells. To give an impression of a typical cell of either of the two clusters, a representative KC was chosen for both of them. To this end, an imaginary center cell, the centroid, for each cluster was determined by calculating the median for each of the parameters (table 3.2). The KC closest to the centroid is shown as a representative for the respective cluster (figure 3.13C).

To evaluate a cluster's homogeneity, silhouette values can be calculated for each data point and plotted in de-, or increasing order as a so-called silhouette plot (Rousseeuw, 1987). The silhouette value is a measure of how well a member of a cluster fits to its own cluster in comparison to how well it fits to the neighboring cluster in terms of Euclidean distance. It can take values between 1 and -1 , with 1 being a perfect fit to the own and -1 a perfect fit to the neighboring cluster. Values around zero indicate an

Figure 3.13. Subdivision of $y$ Kenyon cells by hierarchical cluster analysis into two 'main'clusters.

A) Euclidean distances between cells or clusters of cells are shown as a dendrogram that was created based on Ward's method of minimum variance using the hclust function of R with ward.D2 as a clustering method and Euclidean distances as an input matrix. Input variables were the axon length, the number of axon nodes, the dendrite length, and the number of dendritic claws. All variables were scaled to the respective standard variation to ensure equal contribution of each variable to the clustering. The dendrogram was subdivided into two 'main' clusters that are indicated by color. Indices of individual cells are shown below the dendrogram, color coded to a $\mathrm{min} / \mathrm{max}$ scale that is seen on the left.

B) Statistical comparison of the four input variables used for the cluster analysis, i.e. the axon length, the number of axon nodes, the dendrite length, and the number of dendritic claws between the two clusters highlighted in the dendrogram. The boxes are colored accordingly. They indicate 1st quartile, median, and 3rd quartile, notches the $95 \%$ confidence intervals of the median. The small rectangle within the box represents the mean. Whiskers indicate 5 and 95 percentiles. ${ }^{*}$ indicates significance $p<0.05,{ }^{* * *} p<0.001$, Mann-Whitney $U$ test or Student's $t$ test were used dependent on whether that data were normally distributed (Shapiro-Wilk test), followed by post hoc Bonferroni correction for multiple comparisons.

C) Images show calices and lobes of the two cells that are closest to the median centers of the respective cluster, which is indicated by the color of the scale bars. Scale $=30 \mu \mathrm{m}$.

D) Silhouette plot of the cells belonging to the two clusters colored according to $A$. The silhouette value indicates how well a cell fits to its respective cluster in terms of Euclidean distance in comparison to the neighboring cluster. A value of 1 meaning a $100 \%$ fit to the cluster. If the bar is pointed upwards the cell is closer to the orange cluster, if it is pointed downward the cell is closer to the cyan cluster. 


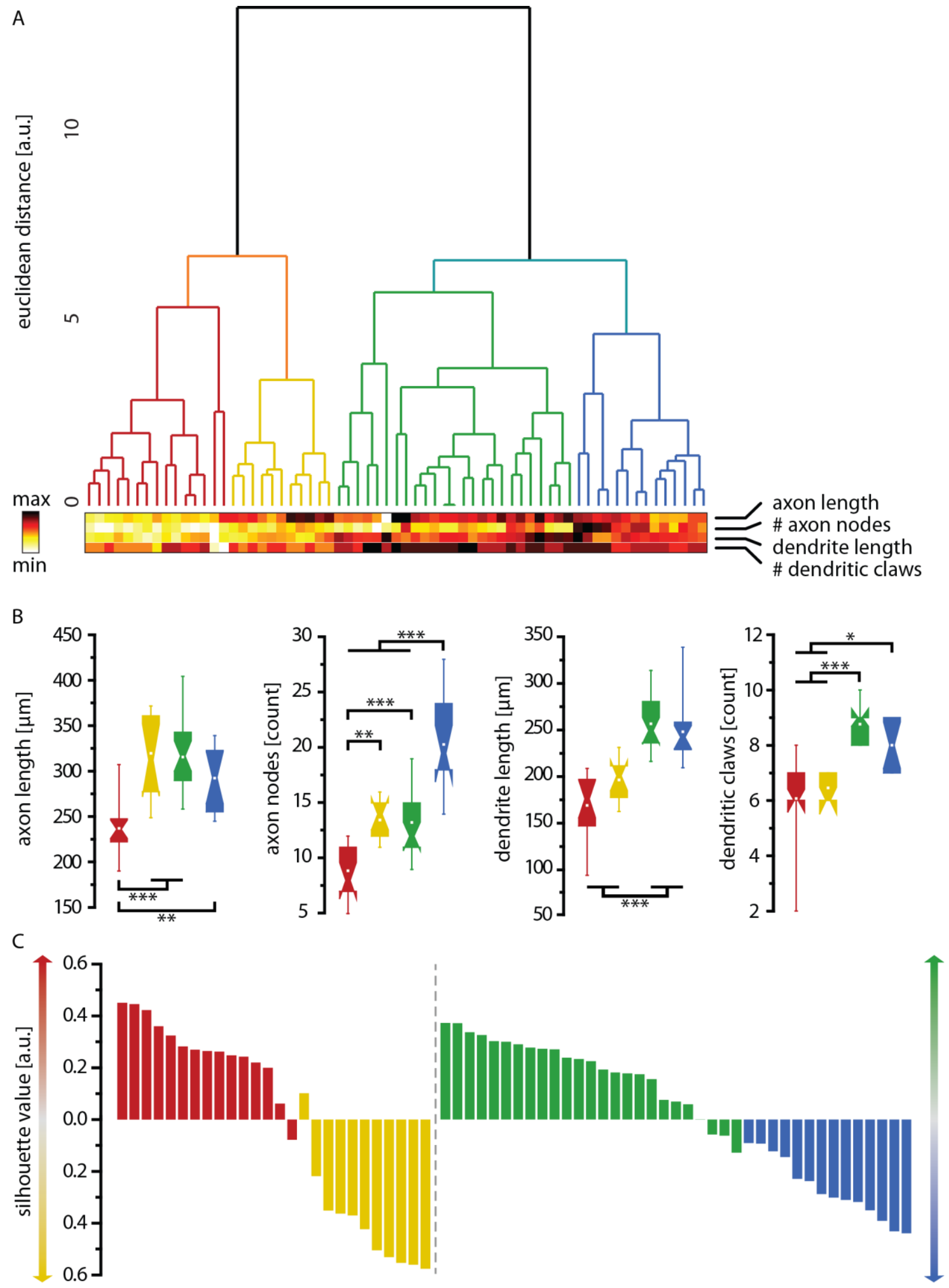

Figure 3.14. Subdivision of $y$ Kenyon cells by hierarchical cluster analysis into four 'sub' clusters. Continued on next page. 
insufficient clustering. Evaluation by silhouettes of the two cluster obtained by hierarchical cluster analysis could show that most of the KCs fit well into their own cluster, with only a few cells taking low or negative values (figure 3.13D). Most of the silhouette values are between 0.2 and 0.5 .

Further analysis of the clustering tree indicated that the two 'main' clusters are composed of two 'sub' clusters each (figure 3.14). Indeed, investigation of the four 'sub' clusters revealed significant differences in respect to the input parameters. The larger of the two 'main' clusters, consisting of bigger neurons, can be subdivided into
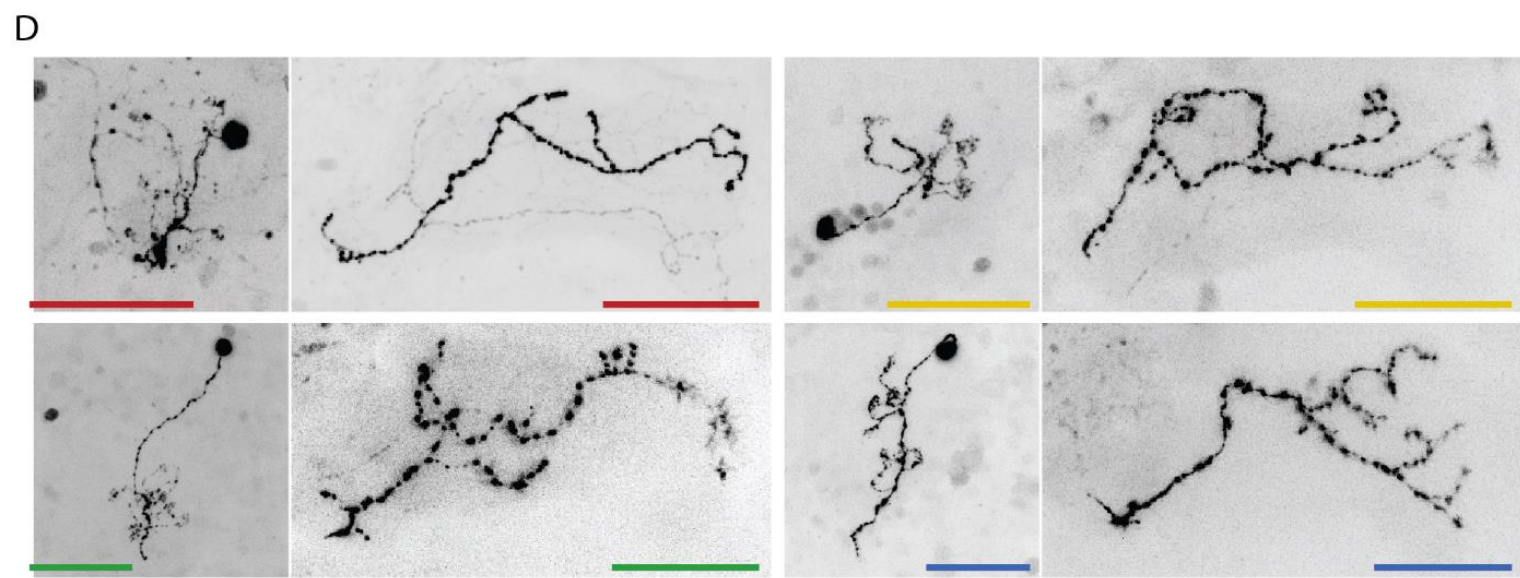

Figure 3.14. Subdivision of $y$ Kenyon cells by hierarchical cluster analysis into four 'sub' clusters.

A) Euclidean distances between cells or clusters of cells are shown as a dendrogram that was created based on Ward's method of minimum variance using the hclust function of R with ward.D2 as a clustering method and Euclidean distances as an input matrix. Input variables were the axon length, the number of axon nodes, the dendrite length, and the number of dendritic claws. All variables were scaled to the respective standard variation to ensure equal contribution of each variable to the clustering. The dendrogram was subdivided into four 'sub' clusters that are indicated by color (compare figure 3.13\#). Indices of individual cells are shown below the dendrogram, color coded to a $\mathrm{min} / \mathrm{max}$ scale that is seen on the left.

B) Statistical comparison of the four input variables used for the cluster analysis, i.e. the axon length, the number of axon nodes, the dendrite length, and the number of dendritic claws between the four clusters highlighted in the dendrogram. The boxes are colored accordingly. They indicate 1st quartile, median, and 3rd quartile, notches the $95 \%$ confidence intervals of the median. The small rectangle within the box represents the mean. Whiskers indicate 5 and 95 percentiles. ${ }^{*}$ indicates significance $p<0.05,{ }^{* * *} p<0.001$, Kruskal-Wallis test or ANOVA were used dependent on whether the data were normally distributed (Shapiro-Wilk test), followed by post hoc Bonferroni correction for multiple comparisons.

C) Silhouette plot of the cells belonging to the four clusters colored according to A. The silhouette value indicates how well a cell fits to its respective cluster in terms of Euclidean distance in comparison to the neighboring cluster. In this case the red colored and the yellow colored cluster, and the green colored and the blue colored cluster are neighboring clusters. A value of 1 meaning a $100 \%$ fit to the cluster. If the bar is pointed upwards the cell is closer to the red colored or green colored cluster respectively, if it is pointed downward the cell is closer to the yellow colored or blue colored cluster respectively.

D) Images show calyces and lobes of the four cells that are closest to the median centers of the respective cluster, which is indicated by the color of the scale bars. Scale $=$ \#\# 
two 'sub' clusters that are significantly different in the number of axonal nodes, though, the two clusters are equal regarding the number of dendritic claws, the dendrite length, and the axon length (figure 3.14B). Accordingly, examination of the silhouette values indicated many KCs with values below 0.2 (figure 3.14C).

Investigation of the smaller of the two 'main' clusters revealed another two 'sub' clusters that are significantly different in two parameters; that is the axon length and the number of axon nodes (figure 3.14B). There is one 'sub' cluster that is short in respect to all four parameters, and another 'sub' cluster that is equal in respect to the calycal parameters quantified, but more similar to the big cluster regarding axonal parameters. Accordingly, the two 'sub' clusters of the smaller 'main' cluster appear well separated looking at the silhouette values, with only a few cells having silhouette values below 0.2 (figure 3.14C).

As for the two 'main' clusters, median centroids cells were calculated for each of the four 'sub' clusters (table 3.2). The $\mathrm{KC}$ of a respective cluster that is closest to the centroid, was chosen as a representative cell. All four representative cells are shown in figure 3.14D.

Table 3.2. Indices of all cluster centroids and the respective closest Kenyon cell in terms of Euclidean distance (nearest neighbor)

\begin{tabular}{|c|c|c|c|c|c|c|}
\hline & $\begin{array}{l}\text { 'main'- } \\
\text { cluster } \\
\text { "orange" }\end{array}$ & $\begin{array}{l}\text { 'main'- } \\
\text { cluster } \\
\text { "cyan" }\end{array}$ & $\begin{array}{l}\text { 'sub'- } \\
\text { cluster } \\
\text { "red" }\end{array}$ & $\begin{array}{l}\text { 'sub'- } \\
\text { cluster } \\
\text { "yellow" }\end{array}$ & $\begin{array}{l}\text { 'sub'- } \\
\text { cluster } \\
\text { "green" }\end{array}$ & $\begin{array}{l}\text { 'sub'- } \\
\text { cluster } \\
\text { "blue" }\end{array}$ \\
\hline & centroid & centroid & centroid & centroid & centroid & centroid \\
\hline $\begin{array}{l}\text { median axon } \\
\text { length }[\mu \mathrm{m}]\end{array}$ & 247.9 & 314.6 & 235.7 & 311.9 & 316.4 & 292.6 \\
\hline $\begin{array}{l}\text { median axon } \\
\text { nodes [count] }\end{array}$ & 11.0 & 15.0 & 8.0 & 14.0 & 12.0 & 19.5 \\
\hline $\begin{array}{l}\text { median } \\
\text { dendrite length } \\
{[\mu \mathrm{m}]}\end{array}$ & 182.6 & 244.2 & 175.1 & 200.1 & 248.8 & 241.7 \\
\hline \multirow{2}{*}{$\begin{array}{l}\text { median } \\
\text { dendritic claws } \\
\text { [count] }\end{array}$} & 6.0 & 8.0 & 6.0 & 6.0 & 9.0 & 8.0 \\
\hline & $\begin{array}{c}\text { nearest } \\
\text { neighbor }\end{array}$ & $\begin{array}{c}\text { nearest } \\
\text { neighbor }\end{array}$ & $\begin{array}{c}\text { nearest } \\
\text { neighbor }\end{array}$ & $\begin{array}{c}\text { nearest } \\
\text { neighbor }\end{array}$ & $\begin{array}{c}\text { nearest } \\
\text { neighbor }\end{array}$ & $\begin{array}{c}\text { nearest } \\
\text { neighbor }\end{array}$ \\
\hline $\begin{array}{l}\text { median axon } \\
\text { length }[\mu \mathrm{m}]\end{array}$ & 227 & 333.8 & 246.3 & 341.4 & 315.1 & 286.1 \\
\hline $\begin{array}{l}\text { median axon } \\
\text { nodes [count] }\end{array}$ & 10 & 14 & 8 & 14 & 12 & 18 \\
\hline $\begin{array}{l}\text { median } \\
\text { dendrite length } \\
{[\mu \mathrm{m}]}\end{array}$ & 202.4 & 227.5 & 181 & 212.1 & 249 & 233.3 \\
\hline $\begin{array}{l}\text { median } \\
\text { dendritic claws } \\
\text { [count] }\end{array}$ & 6 & 8 & 6 & 6 & 9 & 8 \\
\hline
\end{tabular}



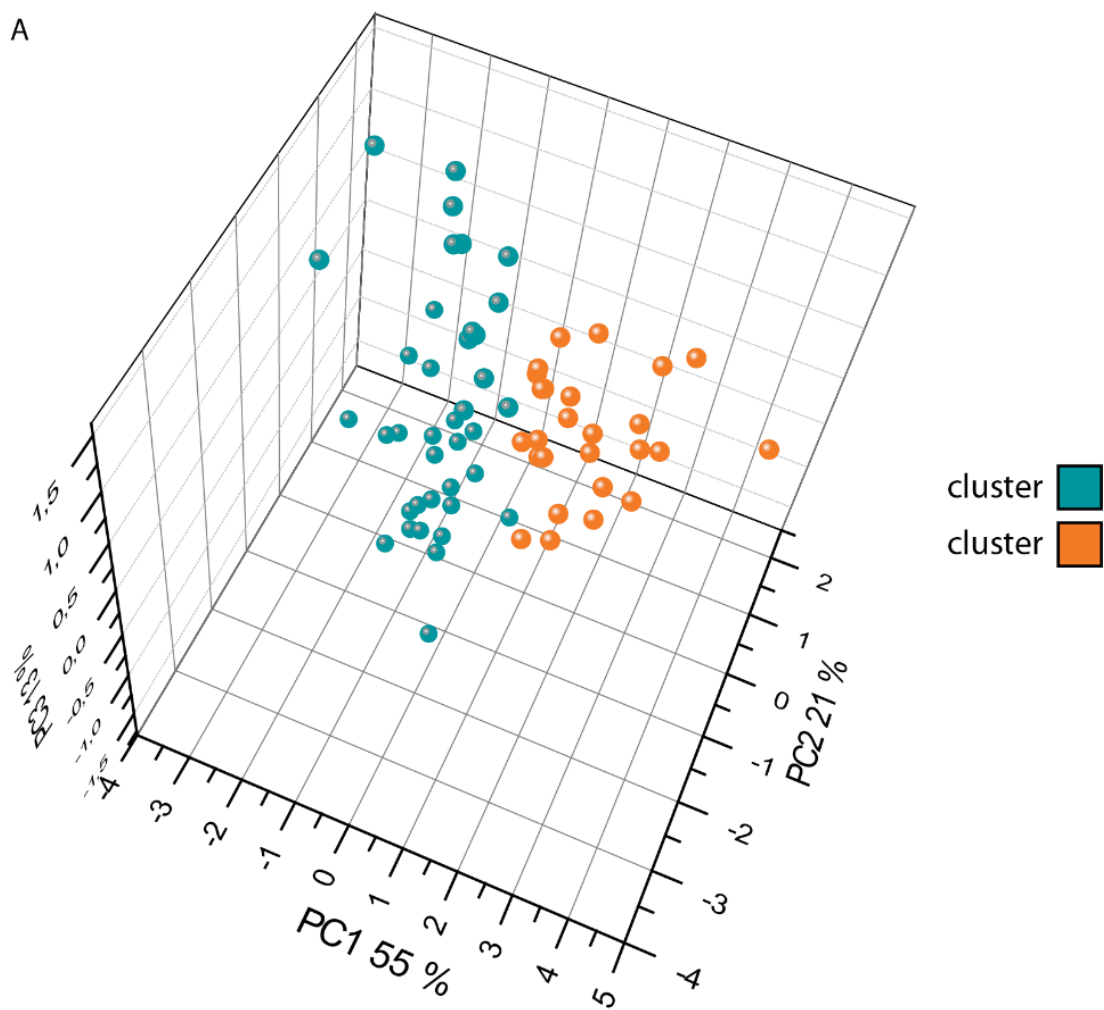

B

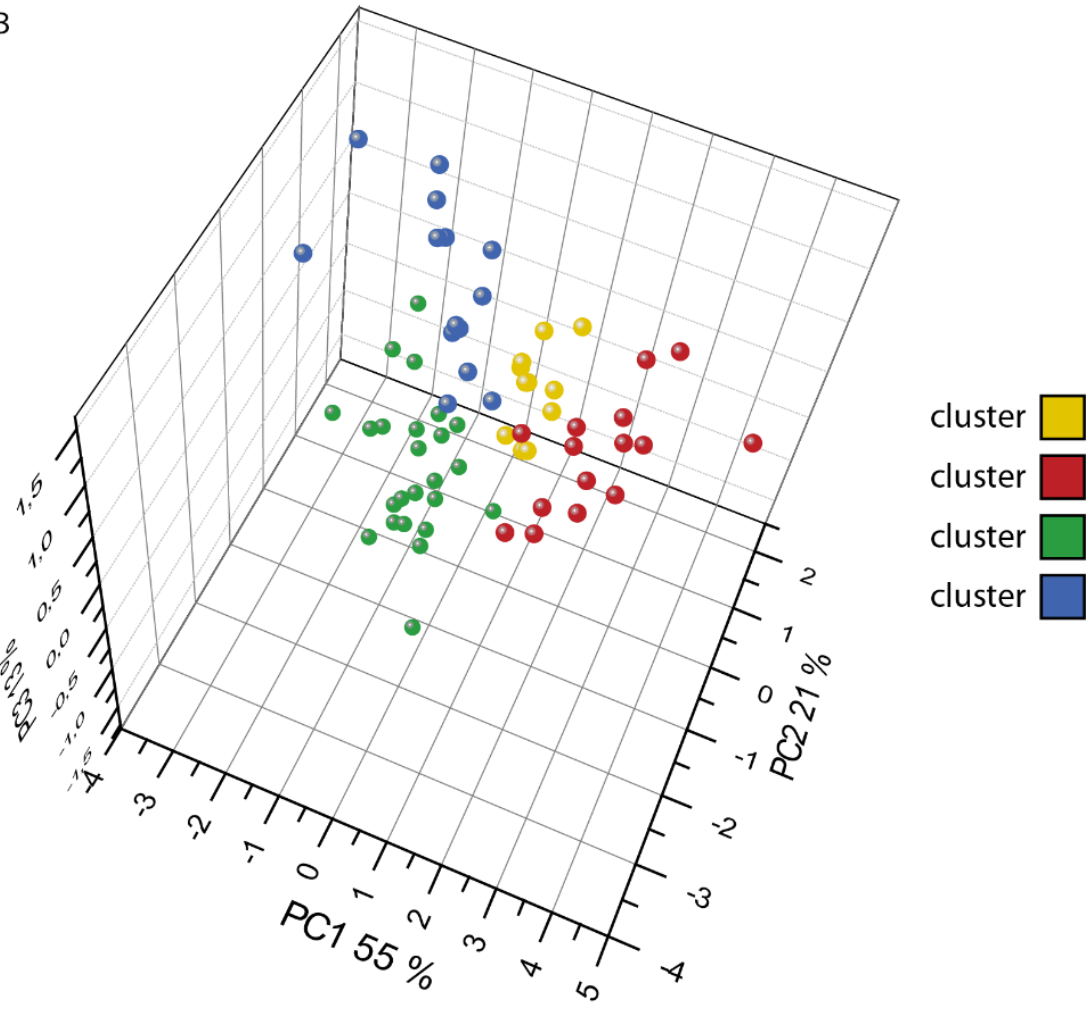

Figure 3.15. Visualization of clusters in three dimensions by principle component analysis.

The clusters attained by hierarchical cluster analysis are shown in principle component space. Axes indicate the first three principle components and the respective percentage of covered variance. Analyzed parameters describing gross $y$ cell morphology were the number of dendritic claws, the dendrite and axon length, and the number of axon nodes.

A) Kenyon cells in principle component space are color coded according to the two 'main' clusters (see figure 3.13)

B) Kenyon cells in principle component space are color coded according to the four 'sub' clusters (see figure 3.14) 
In order to visualize the two 'main' and the four 'sub' clusters, principle component analysis was employed. To this end, the first three principle components were plotted in three dimensional space and color coded according to their cluster affiliation (figure 3.15). Overall, the KCs are equally distributed within principle component space. However, regarding the two 'main' clusters a clear separation between could be seen (figure 3.15A). Though not as strong, the four 'sub' cluster also appear separated in principle component space (figure 3.15B). Thus, principle component analysis confirmed the results obtained by cluster analysis. Y KCs can be clustered into up to four groups that are different in respect to the chosen morphological parameters, i.e. the number of dendritic claws, the dendrite length, the axon length, and the number of axon nodes.

3.2.2.3 Analysis of Kenyon cell gross morphological identity in respect to time of development

It has been shown that genetically different $\mathrm{KC}$ types are produced sequentially by four different MB neuroblasts (Ito et al., 1997; Lee et al., 1999). Therefore, it is possible that also the morphologically different $\mathrm{Y}$ KC clusters found in this study differ in respect to their time of development. Due to the use of MARCM the time of development of each $\mathrm{KC}$ can be traced back to the time when the animal was heat shocked during larval stage (Lee et al., 1999). The 'main' as well as the four 'sub' clusters were compared statistically according to the average time point of heat shock (figure 3.16A). However, no significant difference between neither the two 'main' clusters nor between the four 'sub' clusters could be detected. It was noticed though, that the 'sub' cluster biggest in respect to all four parameters contains many cells of animals that were heat shocked around 48 hrs after larval hatching.

In addition to the statistical comparison, KCs were compared visually in principle component space by being color coded according to when the animals the KCs derived from were heat shocked (figure 3.16B). In accordance to the statistical finding KCs originating from all heat shock time points are equally distributed within principle component space. In conclusion, y KC morphology is independent of the time of the KCs development.

\subsubsection{Quantitative description of Kenyon cell axonal arborizations}

The lobes of the MBs constitute the main output region of the KCs, and are presumably the site of associative olfactory learning (Fiala, 2007; Heisenberg, 2003; Perisse et al., 2013a). Furthermore, the MB Y lobes are differently innervated by MB output neurons and by groups of neurons releasing different types of biogenic amines in a 

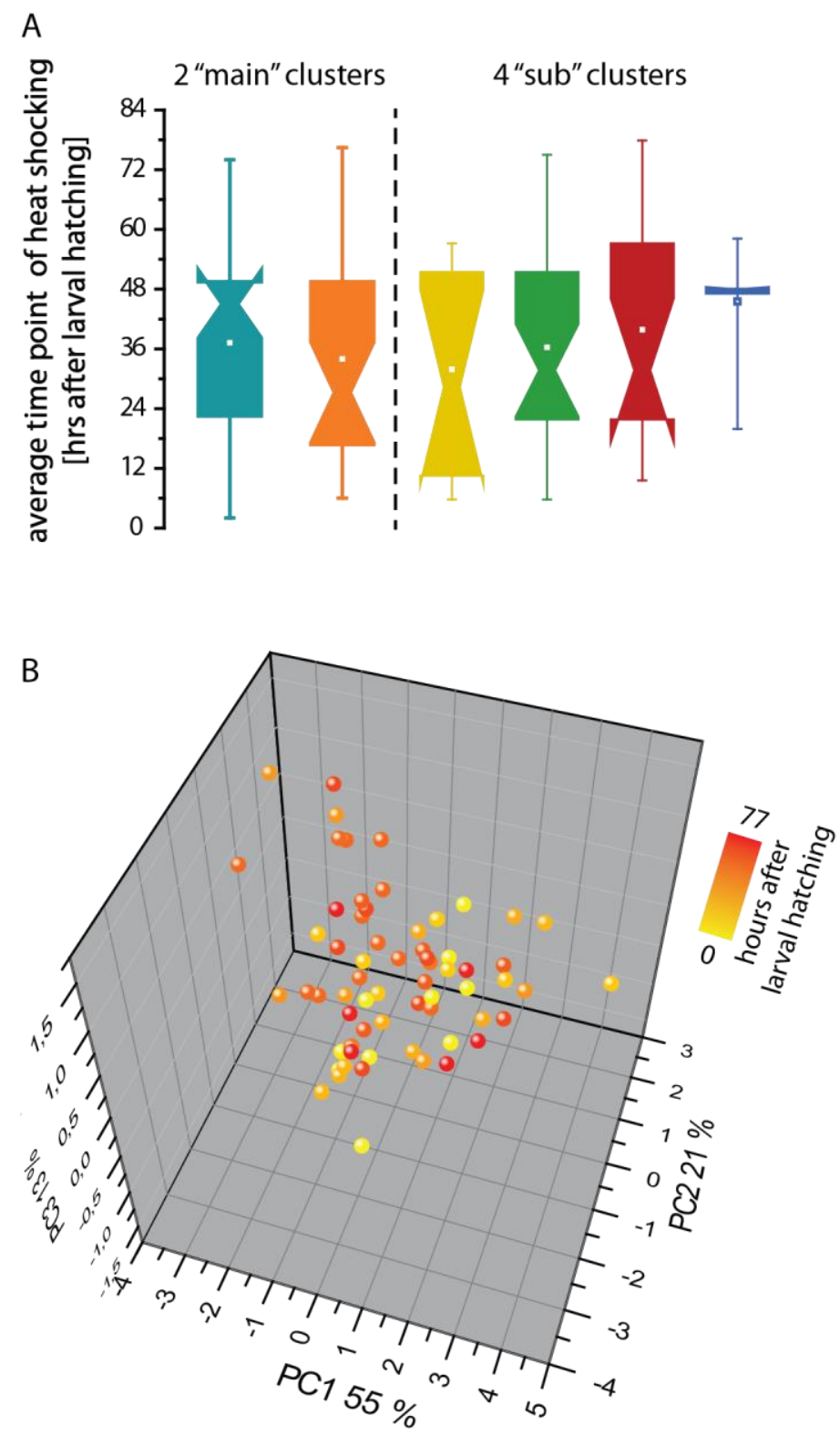

Figure 3.16. Analysis of $y$ Kenyon cell morphology in respect to time of development. The different $y$ cell types attained by hierarchical cluster analysis were compared according to their time of development. Each Kenyon cells birth time was determined as the average time point of heat shock to the respective larva. There is no correlation between the time when a cell was born and its morphology.

A) Statistical comparison of cell clusters attained by cluster analysis according to the time point of heat shocking after larval hatching. Neither the two 'main' clusters, nor the four 'sub' clusters are significantly different (Students $t$-test or ANOVA with posthoc Bonferroni correction).

B) Kenyon cells visualized in three dimensional principle component space (see figure 3.15) are color coded dependent on when larvae were heat shocked for MARCM induction.

compartment specific manner (Aso et al., 2014a; Pech et al., 2013b; Tanaka et al., 2008). Depending on the site of innervation a different valence might be conveyed to KCs and MB output neurons, biasing behavior towards approach or avoidance (Aso et 
al., 2014b; Hige et al., 2015a; Perisse et al., 2016). In total, the MBs are composed of 15 different compartments, including five compartments in the $\mathrm{y}$ lobes, $\mathrm{Y} 1-5$ (Aso et al., 2014a). It might be that different types of $Y$ neurons differently innervate different $y$ lobe compartments in order to facilitate for example approach or avoidance behavior in different conditions. To test this hypothesis, $\mathrm{KC}$ innervation patterns were quantified by measuring the $\mathrm{KCs}^{\prime}$ arborization length within each of the five $\mathrm{Y}$ lobe
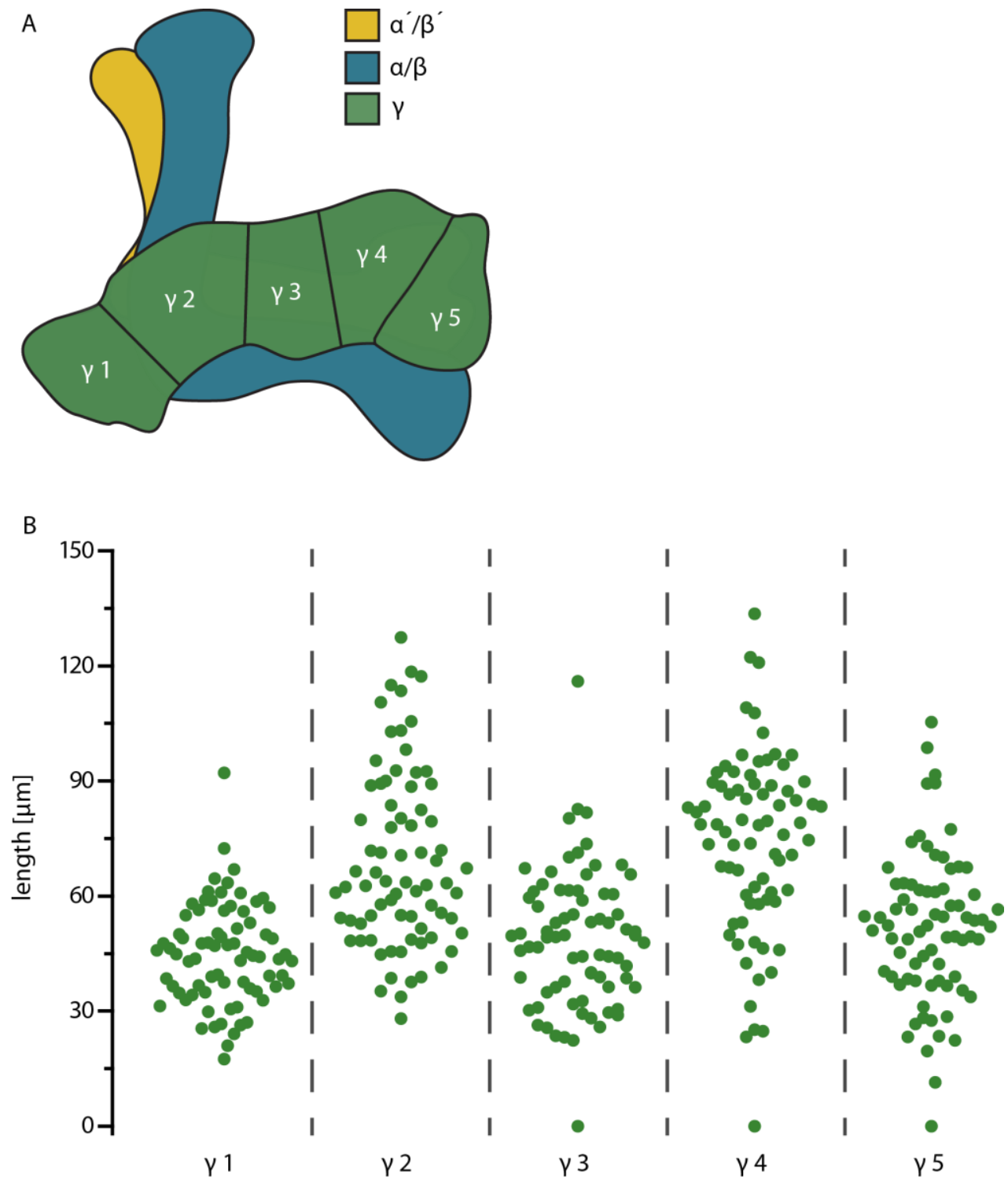

Figure 3.17. Quantification of the axonal arborizations within y compartments 1-5.

The length of arborizations of an axon within a specific $y$ lobe compartment represents to a great deal the connectivity of that cell to extrinsic neurons, i.e. dopaminergic neurons, and mushroom body output neurons, which were shown to innervate the mushroom body lobes in a compartment-specific manner.

A) Illustration of the arrangement of $y$ cell compartments within the $Y$ lobe.

B) Each dot represents the arborizations length of one $Y$ Kenyon cell within the indicated compartment. Between compartment differences regarding the whole population arise from different volumes of the compartments. $\mathrm{N}=75$ cells. 
compartments (figure 3.17), which were identified based on the MB background staining. With the exception of one cell that terminated in $\mathrm{Y} 2$ (figure 3.9A), each $\mathrm{Y} \mathrm{KC}$ developed arborizations in all five $\mathrm{Y}$ lobe compartments. Differences were found to which extent each compartment is innervated. The average length of arborizations within $\mathrm{Y} 1,3$, and $5(44.9 \mu \mathrm{m}, 48.8 \mu \mathrm{m}$, and $51.7 \mu \mathrm{m}$ respectively) were shorter than in $\mathrm{\gamma}^{2}$, and $\mathrm{\gamma} 4(69.0 \mu \mathrm{m}$, and $74.0 \mu \mathrm{m}$ respectively).

Moreover, it was tested if there are simple relationships between compartments. It might be that KCs that arborize relatively strong in one compartment would arborize also relatively strong, or if negatively coupled, relatively weak, in another compartment. This kind of relationship might, for example, correlate with MB extrinsic neurons that interconnect $y$ lobe compartments, like one dopaminergic neuron with dendritic arbors in $\mathrm{Y} 1$, and 2 and output termini in $\mathrm{Y} 4$, and the complementary $\mathrm{MB}$

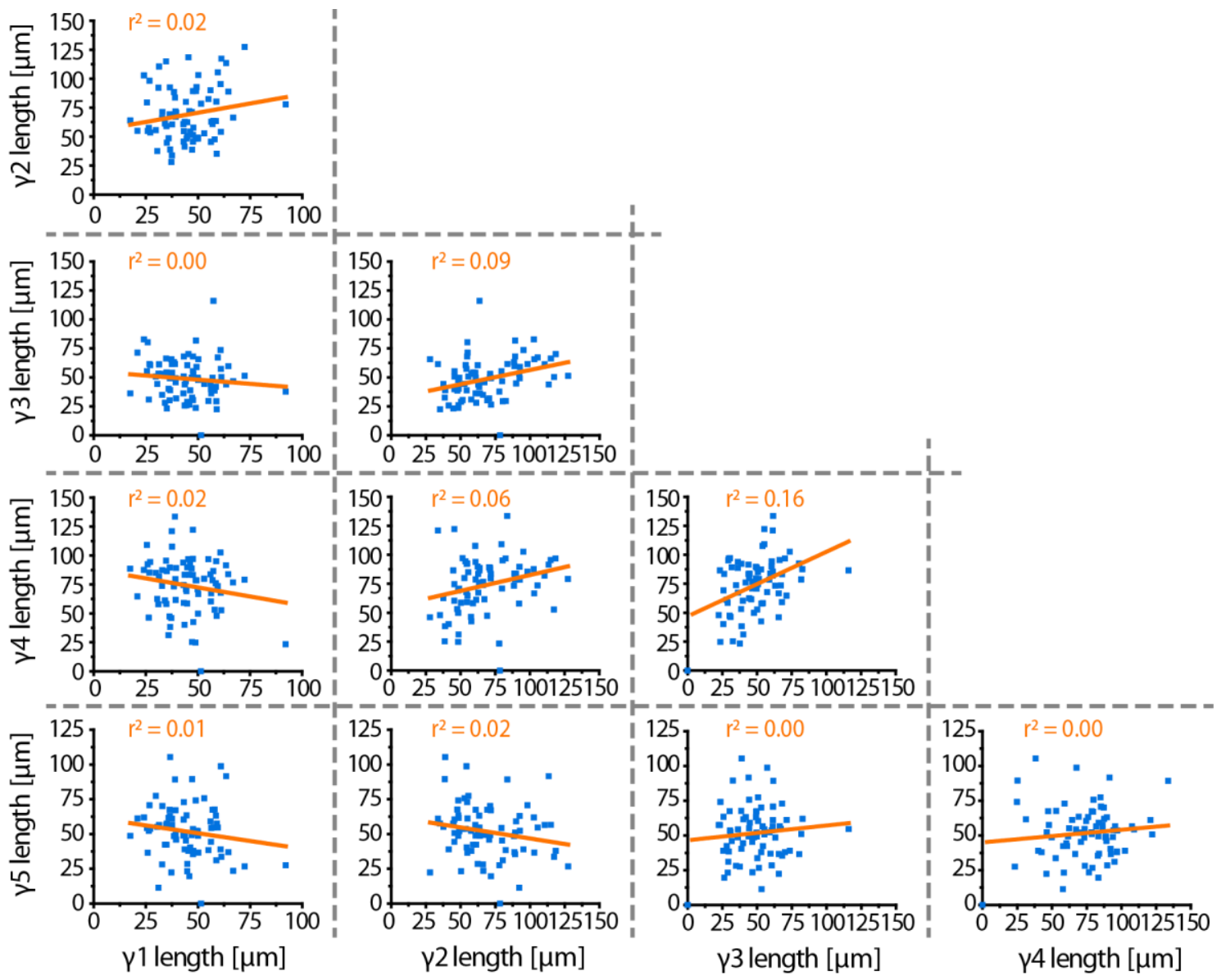

Figure 3.18. Correlations between $\mathrm{Y}$ Kenyon cell arborizations within $\mathrm{Y}$ lobe compartments.

The matrix plot shows the pairwise correlations between $\mathrm{Y}$ lobe arborizations within $\mathrm{Y}$ compartments $1-5$ (see figure 3.17 for single parameter distributions). The correlation between any pair of compartments has been estimated using a simple linear regression model implemented in Origin 8.5. The strength of the correlation is given as the $r^{2}$ value that lies between 0 and 1 . Only linear models between compartments $\mathrm{y}$ 2-4 represent a sufficient fit. 
output neuron with dendritic arbors in $\mathrm{\gamma} 4$ and axonal termini in $\mathrm{\gamma} 1$, and 2 (Aso et al., 2014a). To answer this question, the compartment specific lengths of KC Y lobe arborizations were compared using linear regressions (figure 3.18). Although positive linear fits were identified between $y$ compartments 2-4, overall correlation values (indicated as $r^{2}$ ) are low. Cluster analysis will represent an adequate tool to test if there are specific cell types that rely on multi-compartment-specific arborization patterns.

Table 3.3: Descriptive statistical analysis of $y$ Kenyon cell compartment-specific arborizations

\begin{tabular}{|l|c|c|c|c|c|c|}
\hline & $\min$ & median & $\max$ & mean & SEM & $\mathrm{N}$ \\
\hline $\begin{array}{l}\mathrm{Y} 1 \text { arborizations } \\
{[\mu \mathrm{m}]}\end{array}$ & 17.5 & 44.8 & 92.1 & 44.9 & 1.5 & 75 \\
\hline $\begin{array}{l}\mathrm{Y} 2 \text { arborizations } \\
{[\mu \mathrm{m}]}\end{array}$ & 28 & 63.4 & 127.4 & 69.0 & 2.6 & 75 \\
\hline $\begin{array}{l}\mathrm{Y3} \text { arborizations } \\
{[\mu \mathrm{m}]}\end{array}$ & 0 & 49.3 & 116 & 48.8 & 2.0 & 75 \\
\hline $\begin{array}{l}\mathrm{Y} 4 \text { arborizations } \\
{[\mu \mathrm{m}]}\end{array}$ & 0 & 78.7 & 133.6 & 74.0 & 2.8 & 75 \\
\hline $\begin{array}{l}\mathrm{Y} 5 \text { arborizations } \\
{[\mu \mathrm{m}]}\end{array}$ & 0 & 52.3 & 105.3 & 51.7 & 2.2 & 75 \\
\hline
\end{tabular}

\subsubsection{Descriptive statistical analysis of $y$ Kenyon cells' axonal arborizations}

\subsubsection{Subdivision of $y$ Kenyon cells' axonal arborizations by hierarchical clustering}

To analyze arborization patterns of $\mathrm{KCs}$ in different $\mathrm{y}$ lobe compartments, and to identify possible $\mathrm{Y} K \mathrm{KC}$ types that are defined by differently innervating the $\mathrm{Y}$ lobe, hierarchical cluster analysis was performed in accordance to the analysis of gross $\mathrm{Y} \mathrm{KC}$ morphology (see section 3.2.2.2). Input parameters were each cells arborization length within $y$ lobe compartments 1-5. Two clusters were identified as indicated in the dendrogram (figure 3.19A).

The larger cluster, consisting of 48 cells, shows significant shorter arborizations in compartments $\mathrm{y}_{2}, \mathrm{y}_{3}$, and $\mathrm{Y} 4$, in comparison to the smaller cluster, consisting of 27 cells. The arborization length in $\mathrm{\gamma} 1$, and $\mathrm{y} 5$ of the KCs of both clusters is indistinguishable (figure 3.19B). To visualize the differences between the two clusters the median centroid of each cluster was calculated. The $\mathrm{KC}$ closest to the respective centroid was chosen as a representative for the cluster and is depicted in figure 3.19C. In order to investigate homogeneity of both clusters respectively and to 


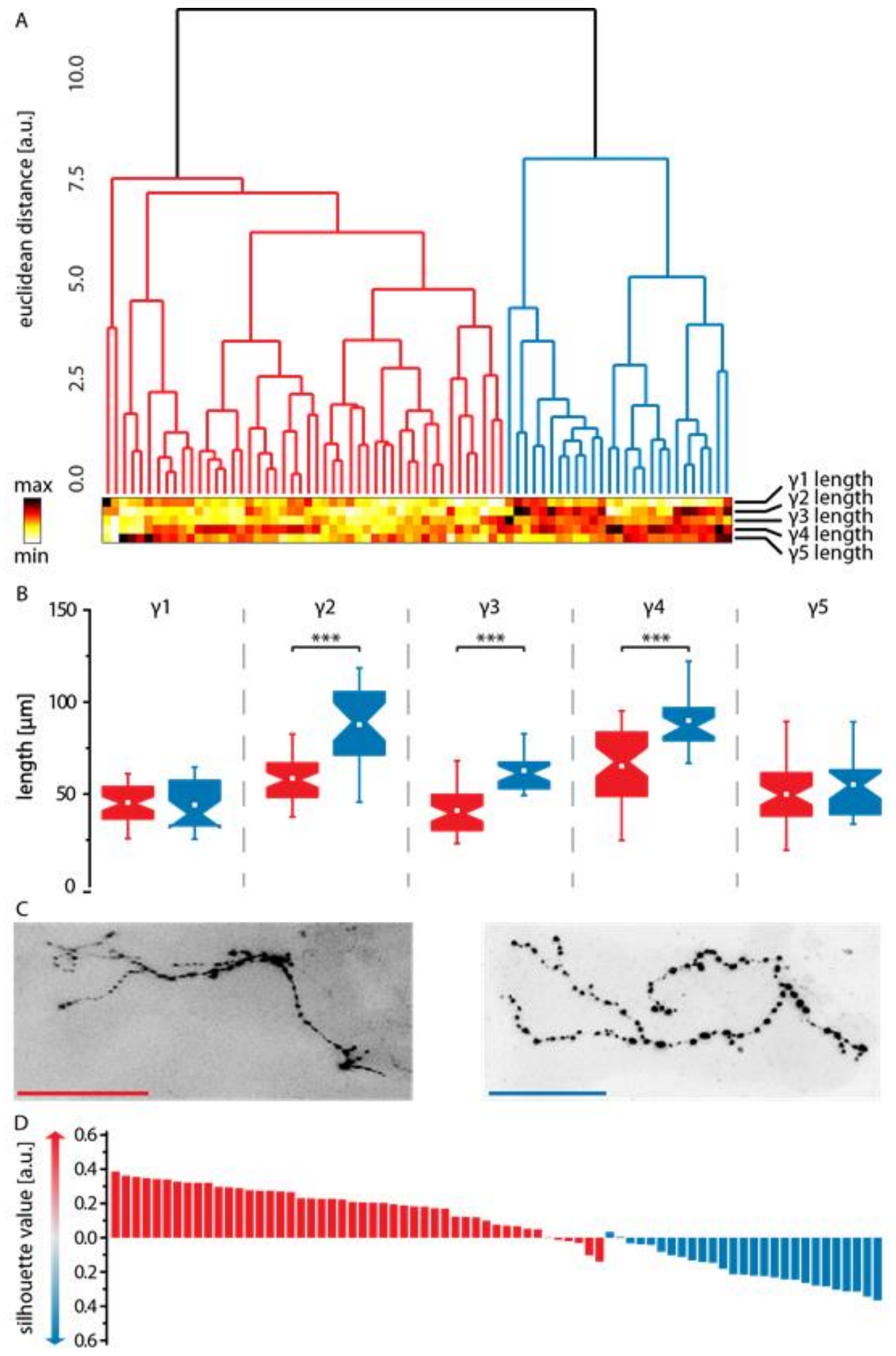

Figure 3.19. Subdivision of $\mathbf{y}$ Kenyon cells by hierarchical cluster analysis based on $y$ compartment innervations. Continued on next page. 
analyze how well the elements of both clusters are separated, a silhouette value for each KC was calculated as described before (section 3.2.2.2 and materials and methods). The silhouette plot shows that the separation of both clusters is not very strict, and many KCs are found with low or negative values (figure 3.19D). Thus, although two different clusters were identified by hierarchical cluster analysis, the difference between the two is rather gradual.

Comparison of the two clusters identified by hierarchical cluster analysis in principle component space showed that relatively many elements of the two clusters intermingle at the border between the two clusters (figure 3.20). However, most of the KCs appear well separated in principle component space. Thus, there is a weak but defined difference between $\mathrm{Y} K C \mathrm{~S}$ in respect to their $\mathrm{Y}$ lobe innervation patterns that is manifested in distinct lengths of arborizations in $\gamma_{2}, 3$, and 4.

\subsubsection{Analysis of Kenyon cell lobular morphological identity in respect to time of development}

Similarly to section 3.2.2.3, the two KC clusters identified by clustering of the lengths of $\mathrm{Y}$ lobe arborizations were analyzed in respect to their time of development. If the KCs lobular morphological identity corresponds to their time of development, then the time when animals were heat shocked to generate the respective $\mathrm{KC}$ clones, should differ among the two $y$ lobe clusters. However, statistical comparison revealed no significant differences between the clusters regarding the average time of heat shock

Figure 3.19 continued.

A) Euclidean distances between cells or clusters of cells are shown as a dendrogram that was created based on Ward's method of minimum variance using the hclust function of R with ward.D2 as a clustering method and Euclidean distances as an input matrix. Input variables were the axonal arborizations of single $y$ cells within $y$ compartments $1-5$. All variables were scaled to the respective standard variation to ensure equal contribution of each variable to the clustering. The dendrogram was subdivided into two clusters that are indicated by color. Indices of individual cells are shown below the dendrogram, color coded to a $\mathrm{min} / \mathrm{max}$ scale that is seen on the left. B) Statistical comparison of the arborizations lengths within $Y$ compartments 1-5 between the two identified clusters highlighted in the dendrogram. The boxes are colored accordingly. They indicate 1st quartile, median, and 3rd quartile, notches the $95 \%$ confidence intervals of the median. The small rectangle within the box represents the mean. Whiskers indicate 5 and 95 percentiles. $* * *$ indicates significance $\mathrm{p}<0.001, * * *$, Mann-Whitney $U$ test or Student's $t$ test were used dependent on whether that data were normally distributed (Shapiro-Wilk test), followed by post hoc Bonferroni correction for multiple comparisons.

C) Images show axonal arborizations of those cells that are closest to the median centers of the respective cluster, which is indicated by the color of the scale bars. Scale $=30 \mu \mathrm{m}$.

D) Silhouette plot of the cells belonging to the two clusters colored according to $A$. The silhouette value indicates how well a cell fits to its respective cluster in terms of Euclidean distance in comparison to the neighboring cluster. A value of 1 meaning a $100 \%$ fit to the cluster. If the bar is pointed upwards the cell is closer to the red colored cluster, if it is pointed downward the cell is closer to the blue colored cluster respectively. 
(figure 3.21A). Additionally, the $\mathrm{KCs}$ were plotted in principal component space according to the length or arborizations in $\mathrm{Y} 1-5$. KCs were color coded dependent on the average time of heat shock applied to the animals that carried the respective cell. In accordance to the statistical comparison, $\mathrm{KCs}$ of animals that received heat shock to a similar point of time were not clustered, but appeared randomly distributed in principle component space (figure 3.21B).

In summary, KCs of the $\mathrm{Y}$ lobe main type develop a great diversity of morphologies. It could be shown that $\gamma$ KCs in similarity to $a / \beta$ and $a^{\prime} / \beta^{\prime} K C s$ bifurcate within the lobe structure and that this point of bifurcation can be taken as a reference to group $\mathrm{Y} \mathrm{KCs}$ into four clusters. Analysis of $\mathrm{y}$ cell morphology revealed that $\mathrm{Y}$ KCs can be clustered into large and small cells that are different in the magnitude of calycal, and axonal arborizations, dendritic claws, and axonal nodes. At last, investigation of the $\mathrm{Y}$ KCs compartment specific arborizations, revealed two $\mathrm{Y}$ cell clusters that differently innervate $y$ lobe compartments $y 2-4$. None of the identified $y$ cell clusters is correlated to the time of the cells' development. Future research can provide answers, however,

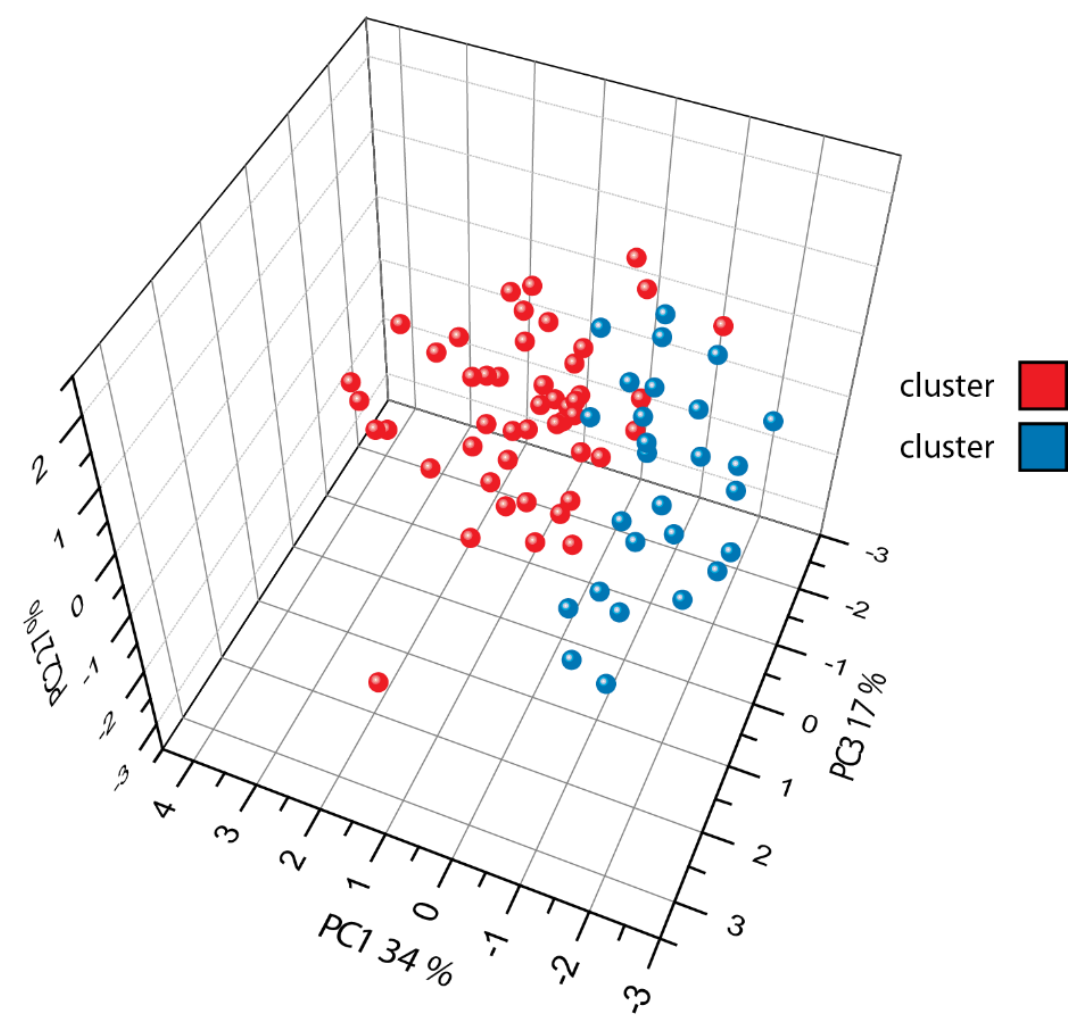

Figure 3.20. Visualization of clusters in three dimensions by principle component analysis.

The clusters attained by hierarchical cluster analysis are shown in principle component space. Axes indicate the first three principle components and the respective percentage of covered variance. Analyzed parameters were the length of arborizations within y compartments 1-5. Single cells are color coded according to their cluster affiliation. 
to whether the identified cell clusters not only share morphological characteristics, but also molecular and/or physiological properties. Nevertheless, morphological analysis of single KCs not only provides a framework for cell type identification but also offers the possibility to investigate the role of structural plasticity of KCs in the context of behavioral adaptation.

A

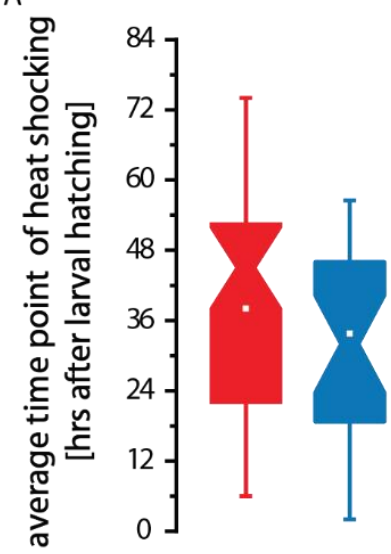

B

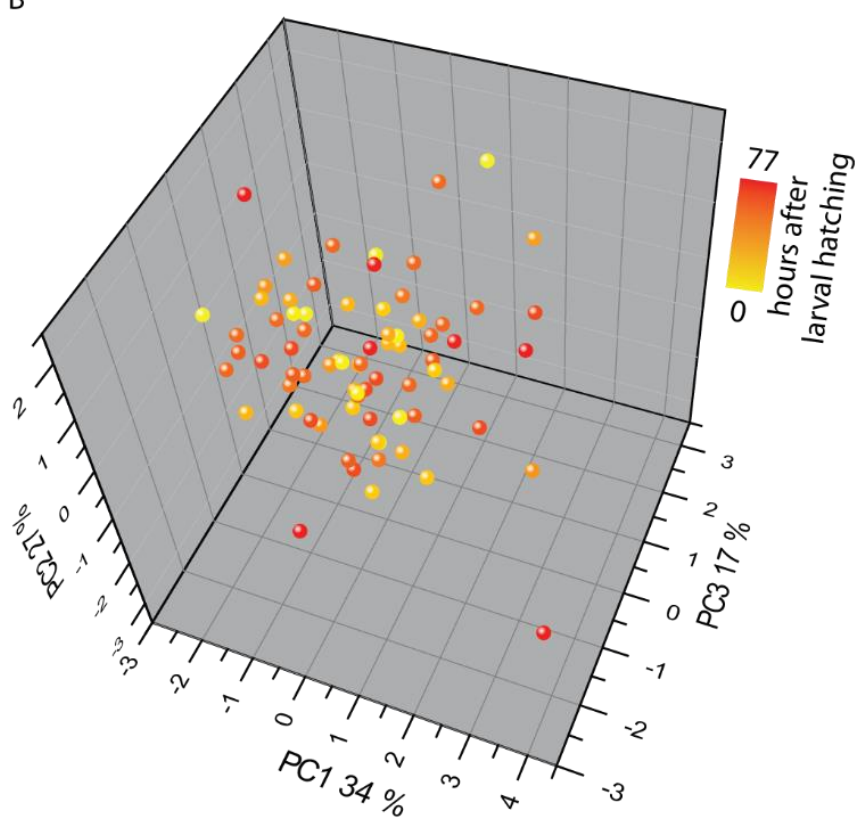

Figure 3.21. Analysis of the arborizations of $y$ Kenyon cells within $y$ compartments 1-5 in respect to time of development.

The two y cell types attained by hierarchical cluster analysis (see figure 3.19) were compared according to their time of development. Each Kenyon cells birth time was determined as the average time point of heat shock to the respective larva. No dependency between the within compartment arborizations and time of birth of a respective $y$ Kenyon cell could be detected.

A) Statistical comparison of cell clusters attained by cluster analysis according to the time point of heat shocking after larval hatching. No significant difference was found (Students $t$-test).

B) Kenyon cells visualized in three dimensional principle component space (compare to figure 3.20) are color coded dependent on when larvae were heat shocked for MARCM induction.

\subsection{Analysis of structural plasticity of individual Kenyon cells}

Although KCs of the Drosophila MBs have for long been implicated in learning and memory processes evidences for plasticity in KCs are still scarce. The comprehensive analysis of $\mathrm{Y}$ KCs in the previous chapters provides a basis for future research on single Y KCs physiological properties and how they contribute to memory formation and retrieval. In the next chapters, the morphology of not only $Y K C s$, but also of $a / \beta$ and $a^{\prime} / \beta^{\prime}$ KCs shall be analyzed in respect to structural plasticity. Structural plasticity was shown in various model systems to underlie long-term behavioral changes (Bailey and 
Chen, 1989; Budnik, 1996; Kozorovitskiy et al., 2005; Xu et al., 2009). The observed neuronal modifications are various and include the outgrowth, or retraction of neurite branches, changes in the number of synapses, changes in synaptic effectiveness, the stabilization of synapses, and alterations in the structure of postsynaptic dendritic spines (for review see for example Bailey and Kandel, 1993, 2008; Bourne and Harris, 2008; Caroni et al., 2012; Greenough and Bailey, 1988; Lamprecht and LeDoux, 2004; Mayford et al., 2012; Sigrist and Schmitz, 2011). In Drosophila, structural plasticity was shown to occur in several neuropils or cell types in response to sleep deprivation, conditions of social enrichment or social isolation, visual or olfactory deprivation in the ventral lateral neurons, the lobula plate, the MBs, and the central complex (Barth and Heisenberg, 1997; Bushey et al., 2011; Donlea et al., 2009, 2014; Heisenberg et al., 1995; Technau, 1984; Yuan et al., 2011).

Moreover, it was found in honey bees that foraging experience had an effect on MB volume and KC fine structure (Farris et al., 2001; Withers et al., 1993), and food deprivation led to the outgrowth of larval Drosophila octopaminergic neurons (Koon et al., 2011). Preliminary results have indicated that keeping flies on different caloric food conditions alters the connectivity between KCs and dopaminergic neurons, as well as

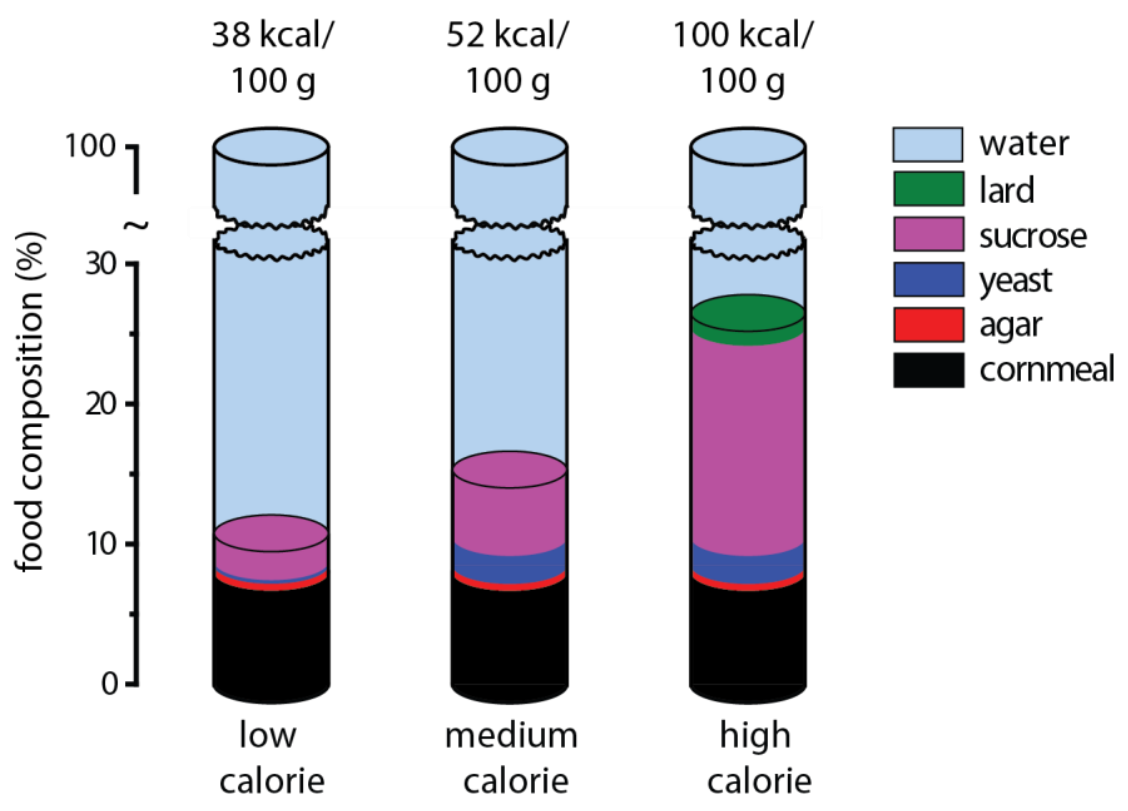

Figure 3.22. Composition of different calorie fly food.

Three different calorie cornmeal diets were prepared for flies for experiments on the structural plasticity of Kenyon cells. All food diets contain the same concentration of cornmeal and agar but differ in water, lard, sucrose, and yeast content. The energy content of each food diet was calculated based on the nutrient value of the ingredients. Low calorie food has $38 \mathrm{kcal} / 100 \mathrm{~g}$, medium calorie food has $52 \mathrm{kcal} / 100 \mathrm{~g}$, and high calorie food has $100 \mathrm{kcal} / 100 \mathrm{~g}$. 
the connectivity between $\mathrm{KCs}$ and $\mathrm{MB}$ output neurons in a compartment specific manner (T. Riemensperger, personal communication). Connectivity was measured using GFP reconstitution across synaptic partners (Feinberg et al., 2008). It seems likely that changes in connectivity are accompanied by KC structural changes. To investigate general mechanisms of structural plasticity in KCs the same feeding conditions as by $\mathrm{T}$. Riemensperger were used to compare single KCs deriving from all major MB lobes. To this end, MARCM flies that expressed hexameric mCherry in single $\mathrm{KCs}$ of different MB lobes via distinct MB driver lines, 5HT1B-Gal4 for the $\mathrm{Y}$ lobe, GMR35B12-Gal4 for the $a^{\prime} / \beta^{\prime}$ lobes, and OK107-Gal4 for the $a / \beta$ lobes, were kept on either low-calorie, medium-calorie, or high-calorie food (figure 3.22) for 7-8 days from 1 day after imago hatching. All flies were raised on standard cornmeal food. Surprisingly, when the weight of the flies was measured before and after they were put on different calorie diet, the flies kept on medium-calorie diet gained significantly more weight than flies kept on low-, and high-calorie diet (figure 3.23). However, differences in body fat content were not measured.

To compare KCs morphologically, the same cell characteristics were quantified as described in section 3.2 for the analysis of $Y$ neurons. Included parameters were the number of dendritic claws, the dendrite, and axon length, and the number of axon

A

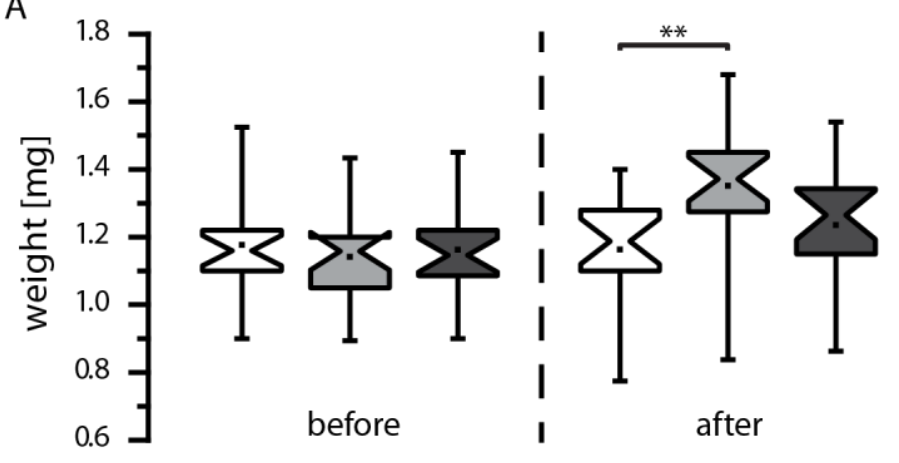

B

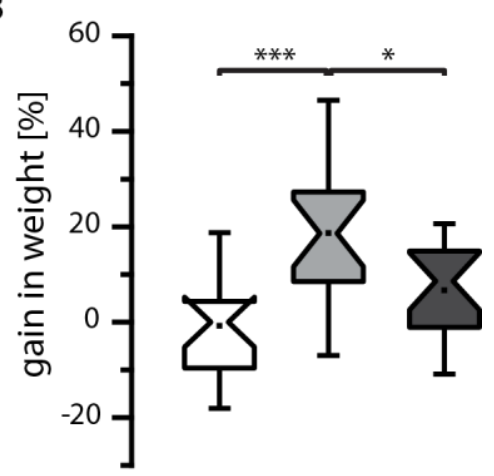

low calorie $\square$ medium calorie $\square$ high calorie

Figure 3.23. Weight measurements of flies kept on different calorie food.

MARCM flies potentially expressing hexameric mCherry in single $y$ Kenyon cells were weighed before and after they were kept for 7-8 days on either low-, medium-, or high-, calorie diet. Group sizes varied between 4 to 17 flies per vial. Flies kept on medium-calorie food gained the most weight.

A) Before being put on different food conditions, 0-1 day old flies had an average weight of $\sim 1.2$ $\mathrm{mg}$ independent of the later diet-class affiliation. After being kept on different nutritious diets, those flies kept on medium-calorie food were significantly heavier than flies kept on low-calorie food.

B) The gain in weight was calculated for each group of flies by division of the post-diet weight by the pre-diet weight. Flies kept on medium-calorie food gained significantly more weight than flies kept on either low-, or high-calorie food. 

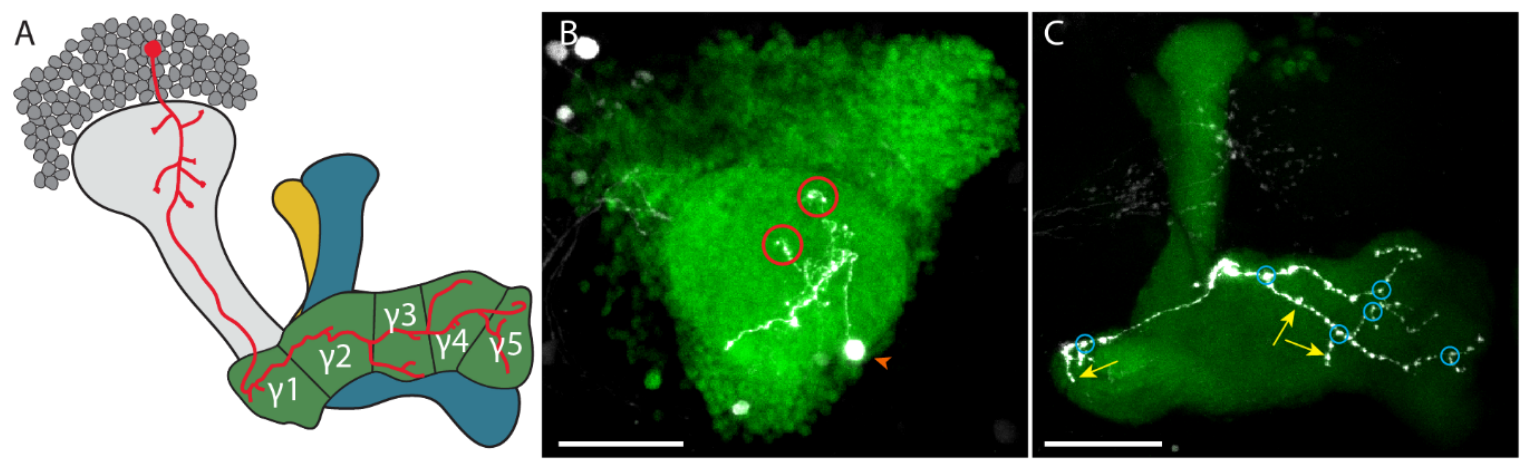

Kenyon cell $\square$ lobe $\square a^{\prime} / \beta^{\prime}$ lobe $\square$ a/ $\beta$ lobe $\bigcirc$ dendritic claw rsoma

A bouton like structure $\bigcirc$ node

D

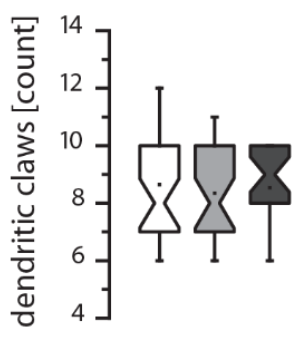

$\mathrm{E}$

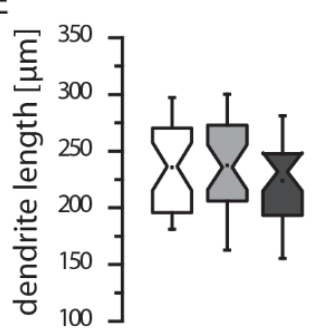

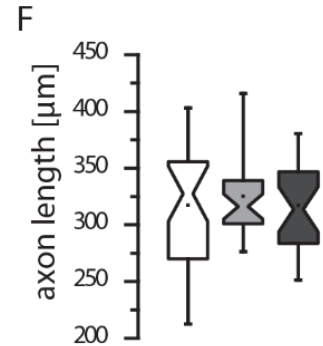

G

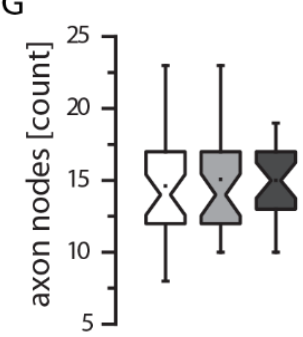

$\mathrm{H}$

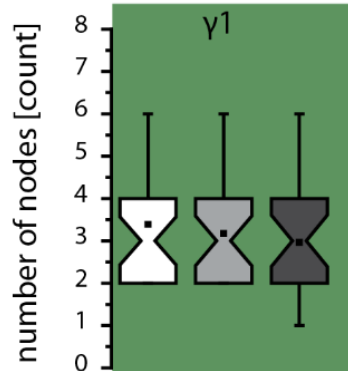

1

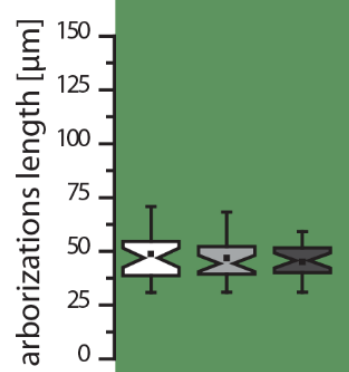

J

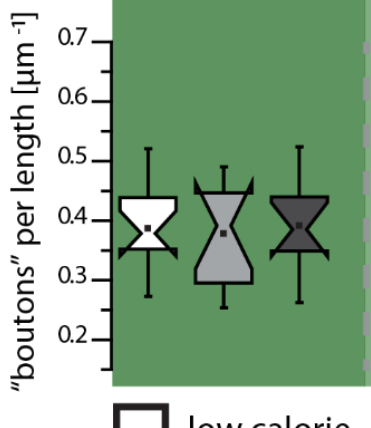

low calorie

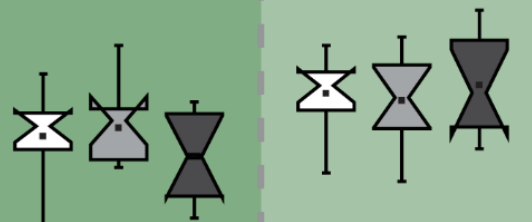

ү5
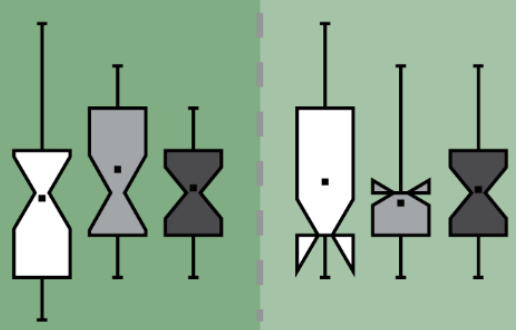

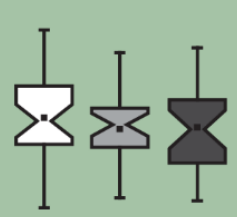

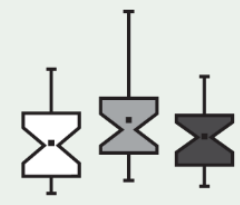

Figure 3.24. Effect of different calorie diets on y cell morphology. Continued on next page. 
nodes. Additionally, the length of arborizations and the number of nodes within the compartments of the respective lobe structure were quantified. The $\mathrm{Y}$ lobe was subdivided in $Y$ compartments $1-5$, the $a^{\prime} / \beta^{\prime}$ lobes in $a^{\prime}$ compartments $1-3$, and $\beta^{\prime}$ compartments $1-2$, and the $a / \beta$ lobes in a compartments $1-3$, and $\beta$ compartments 1 2 (Aso et al., 2014a; Tanaka et al., 2008). Furthermore, if KC confocal images were judged to be of very good quality the number of bouton-like structures per unit length within the different compartments was counted.

Regarding Y KCs, no differences in the cells dendrite length, number of dendritic claws, axon length, and the number of axon nodes (figure 3.24D-G), nor in the arborizations length within $y$ compartments 1-5 (figure 3.24I) were observed between cells deriving from animals kept on different calorie diets. Moreover, the density of bouton-like structures within ү1-5 were not different between flies kept on different calorie diets (figure 3.24J). However, in regard to the number of nodes, a significant higher number was found in the $\mathrm{y} 5$ compartment in $\mathrm{KCs}$ derived from flies kept on medium calorie food in comparison to cells derived from flies kept on low-, and high calorie food (figure $3.24 \mathrm{H})$.

Figure 3.24 continued.

Flies expressing hexameric mCherry in individual y Kenyon cells via the Gal4 driver 5HT1B were kept on different calorie diets for 7-8 days during adulthood. Single cells were compared statistically dependent on the flies' diet and in regard to $y$ cell morphological parameters. From the 19 compared parameters a significant difference was detected only in the number of nodes within the $\mathrm{y} 5$ compartment.

A) Illustration of a $y$ cell and its arborizations within different parts of the mushroom body that were quantified for the statistical analysis of different food diets and their effect on $\mathrm{y}$ Kenyon cell morphology.

$B, C)$ The quantified parameters are highlighted in confocal images of the mushroom body calyx (B) and the mushroom body lobes (C).

$D-G)$ Y Kenyon cells deriving from animals kept on different calorie diets during imago stage were compared statistically in regard to their morphological characteristics within the cells' input and output regions. No significant differences between $y$ cells deriving from flies kept on different calorie diets was detected in the number of dendritic claws (D), the dendrite length (E), the axon length $(F)$, and the number of axon nodes $(G)$.

$\mathrm{H}-\mathrm{J}$ ) Y Kenyon cells deriving from animals kept on different calorie diets during imago stage were compared statistically in regard to their compartment-specific arborizations. A significant difference was detected in the number of nodes within the $\mathrm{y} 5$ compartment of $\mathrm{y}$ cells derived from flies kept on medium-calorie food in comparison to $y$ cells derived from flies kept on low-, or high-calorie food. In compartments $\mathrm{y}^{-4} 4$ no significant difference was detected $(\mathrm{H})$. Regarding the length of arborizations within $\mathrm{Y1-5}$ (I) and the "bouton"-density within $\mathrm{Y1-5}$ (J) no significant difference between $y$ cells deriving from flies kept on different calorie diets was detected.

Data were compared statistically using ANOVA if data were normally distributed or a KruskalWallis test if date were not normally distributed. Normality of the distributions was determined by the Shapiro-Wilk test. $p$-values were corrected for multiple comparisons according to Bonferroni's principle. $*$ is defined as $p<0.05$. 


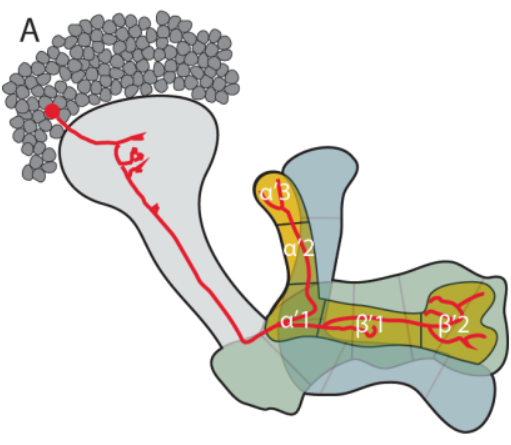

Kenyon cell

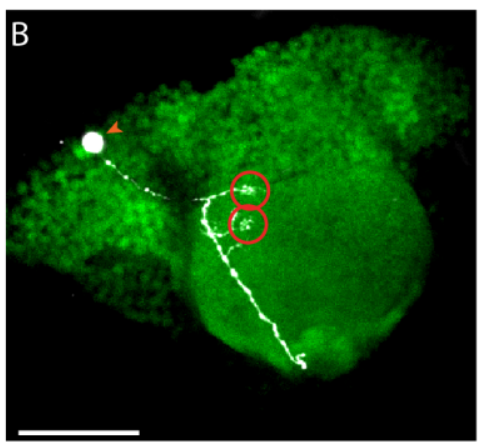

dendritic claw $>$ soma

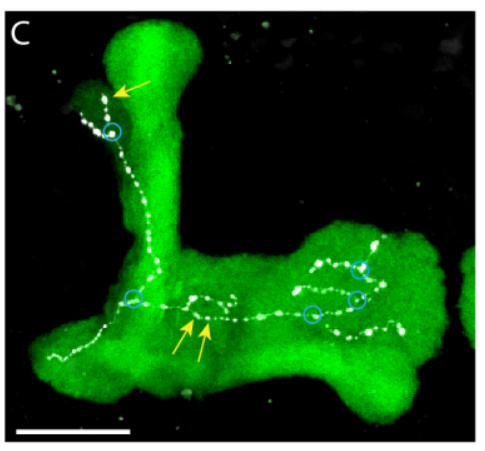

$\nearrow$ bouton like structure $\bigcirc$ node

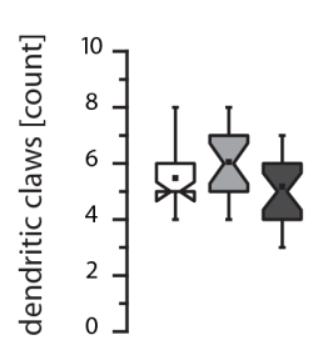

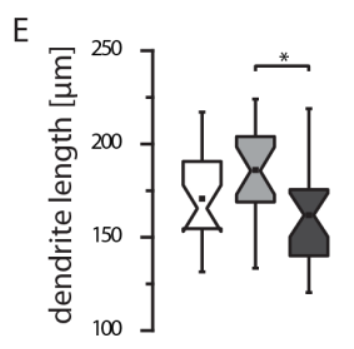
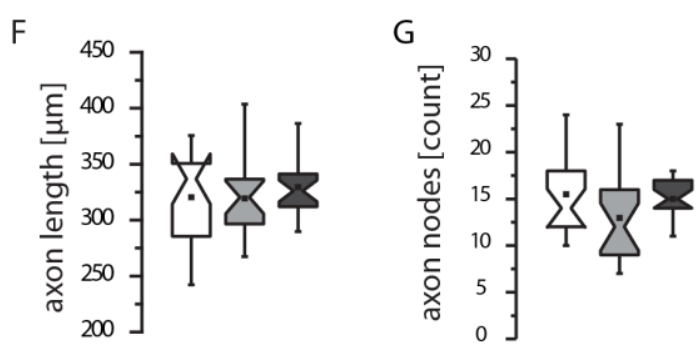

$\mathrm{H}$
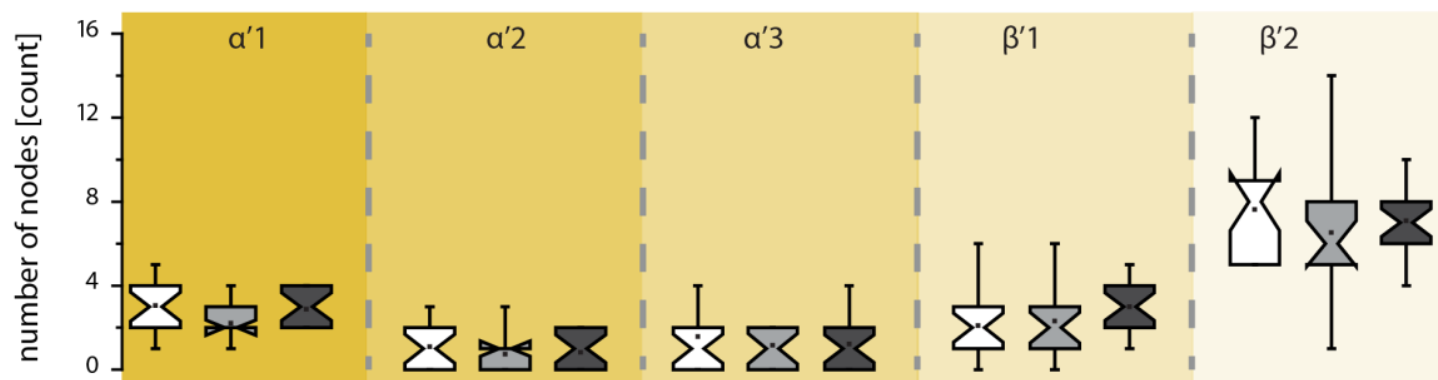

I

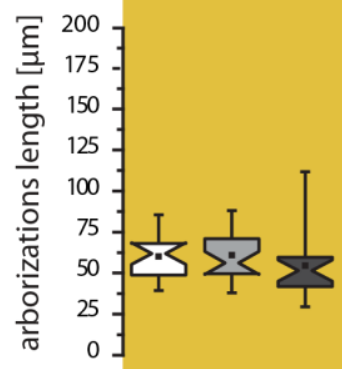

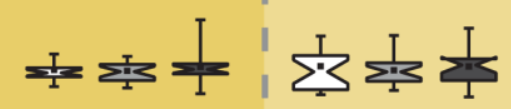

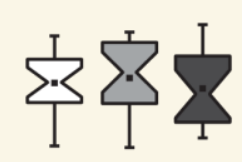

$J=$

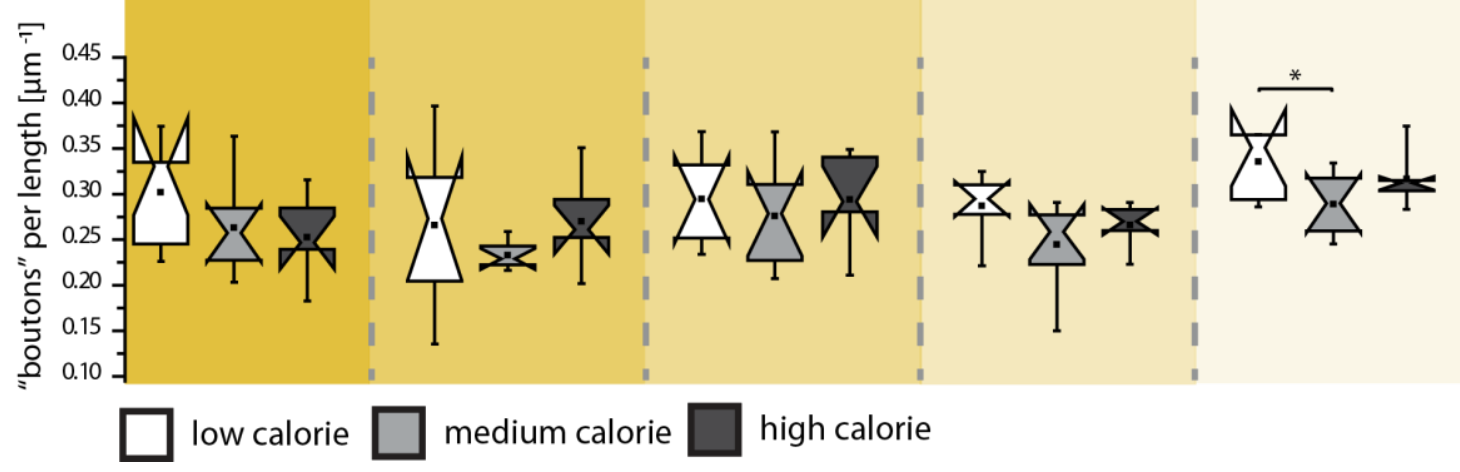

Figure 3.25. Effect of different calorie diets on $\mathbf{a}^{\prime} / \boldsymbol{\beta}^{\prime}$ cell morphology. Continued on next page. 
Analysis of $a^{\prime} / \beta^{\prime} K C$ s revealed that different calorie food diets did not alter the number of dendritic claws (figure 3.25D), the axon length (figure 3.25F), or the number of axon nodes (figure 3.25G). However, dendrites of $a^{\prime} / \beta^{\prime} \mathrm{KCs}$ of flies kept on mediumcalorie food were longer than of flies kept on high-calorie food (figure 3.25E). In regard to compartmental arborizations length and the number of axon nodes within the $a^{\prime} / \beta^{\prime}$ lobes, KCs derived from flies kept on different food diets showed no significant differences (figure $3.25 \mathrm{H}$, I). However, comparison of "bouton"-densities within different $a^{\prime} / \beta^{\prime}$ compartments, indicated a significantly higher density of bouton-like structures in the $\beta^{\prime} 2$ compartment in KCs derived from flies kept on low-calorie food (3.25J) compared to KCs derived from flies kept on medium-calorie food.

$a / \beta$ KCs did not develop significant structural changes independent whether they derived from flies kept on low-, medium-, or high-calorie diet. Parameters analyzed were the number of dendritic claws, the dendrite, and axon length, and the number of axon nodes (figure 3.26D-G). Furthermore, the arborizations length within the $a / \beta$ lobe compartments, the density of bouton-like structures within $a / \beta$ lobe

Figure 3.25 continued.

Flies expressing hexameric mCherry in individual $a^{\prime} / \beta^{\prime}$ Kenyon cells via the Gal4 driver GMR35B12 were kept on different calorie diets for 7-8 days during adulthood. Single cells were compared statistically dependent on the flies' diet and in regard to $a^{\prime} / \beta^{\prime}$ cell morphological parameters. From the 19 compared parameters a significant difference was detected only in the dendrite length and the bouton density within the $\beta^{\prime} 2$ compartment.

A) Illustration of $a a^{\prime} / \beta^{\prime}$ cell and its arborizations within different parts of the mushroom body that were quantified for the statistical analysis of different food diets and their effect on $a^{\prime} / \beta^{\prime}$ Kenyon cell morphology.

$B, C)$ The quantified parameters are highlighted in confocal images of the mushroom body calyx (B) and the mushroom body lobes (C).

$D-G) a^{\prime} / \beta^{\prime}$ Kenyon cells deriving from animals kept on different calorie diets during imago stage were compared statistically in regard to their morphological characteristics within the cells' input and output regions. A significant difference was detected in the dendrite length of $a^{\prime} / \beta^{\prime}$ cells derived from flies kept on medium-calorie food in comparison to $\alpha^{\prime} / \beta^{\prime}$ cells derived from flies kept on high-calorie food (E). No significant differences between $\alpha^{\prime} / \beta^{\prime}$ cells deriving from flies kept on different calorie diets were detected in the number of dendritic claws (D), the axon length (F), and the number of axon nodes $(\mathrm{G})$.

$\mathrm{H}-\mathrm{J}$ ) $\mathrm{a}^{\prime} / \beta^{\prime}$ Kenyon cells deriving from animals kept on different calorie diets during imago stage were compared statistically in regard to their compartment-specific arborizations. A significant difference was detected in "bouton"-density within the $\beta^{\prime} 2$ compartment of $a^{\prime} / \beta^{\prime}$ cells derived from flies kept on low-calorie food in comparison to $a^{\prime} / \beta^{\prime}$ cells derived from flies kept on mediumcalorie food. In compartments $\alpha^{\prime} 1-3$ and $\beta^{\prime} 1$ no significant difference was detected (J). Regarding the number of nodes within $a^{\prime} 1-3$ and $\beta^{\prime} 1-2$ and the arborizations length within $a^{\prime} 1-3$ and $\beta^{\prime} 1-2$ no significant difference between $a^{\prime} / \beta^{\prime}$ cells deriving from flies kept on different calorie diets was detected.

Data were compared statistically using ANOVA if data were normally distributed or a KruskalWallis test if date were not normally distributed. Normality of the distributions was determined by the Shapiro-Wilk test. p-values were corrected for multiple comparisons according to Bonferroni's principle. $*$ is defined as $\mathrm{p}<0.05$ 

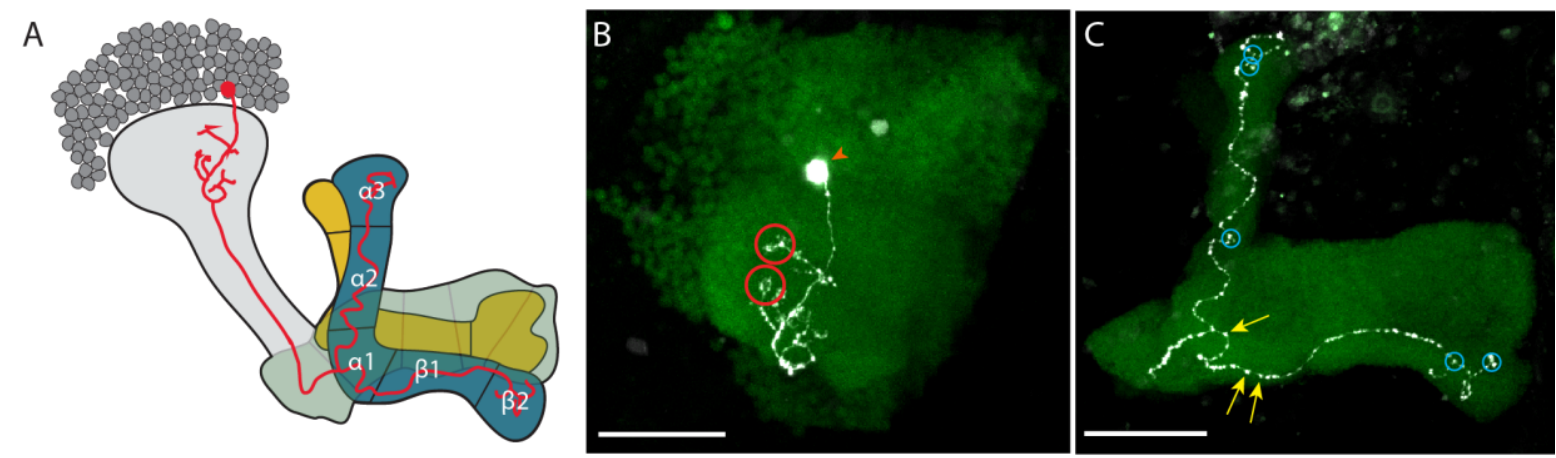

Kenyon cell $\square$ ylobe $\square a^{\prime} / \beta^{\prime}$ lobe $\square$ a/ $\beta$ lobe $\bigcirc$ dendritic claw $\quad$ soma

D

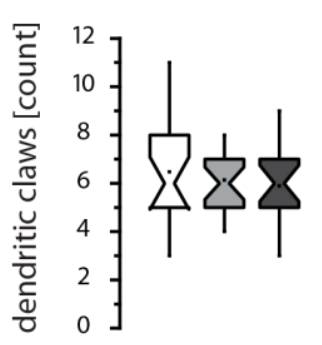

E

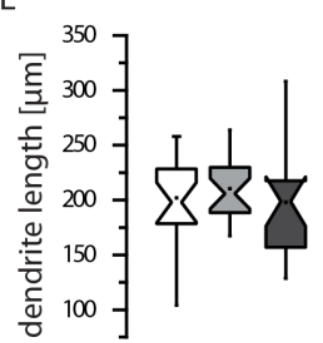

$\mathrm{F}$

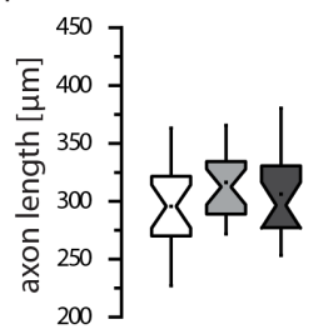

G

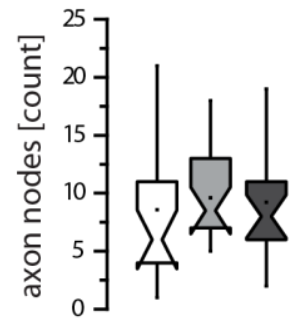

$\mathrm{H}$
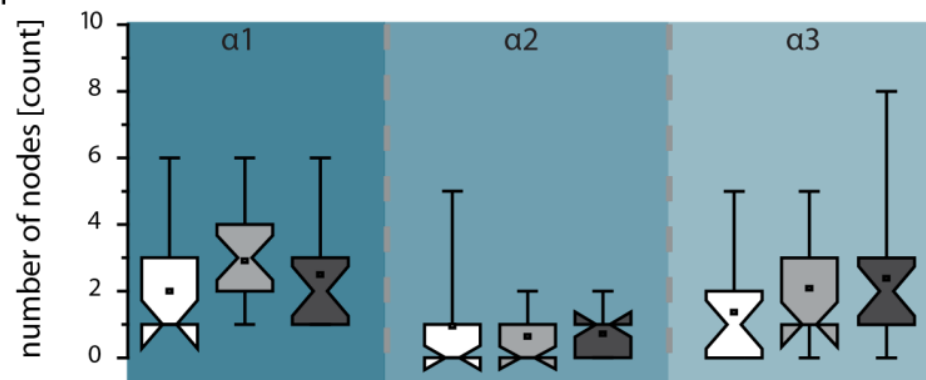

$\beta 1$

$\beta 2$

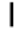

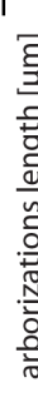
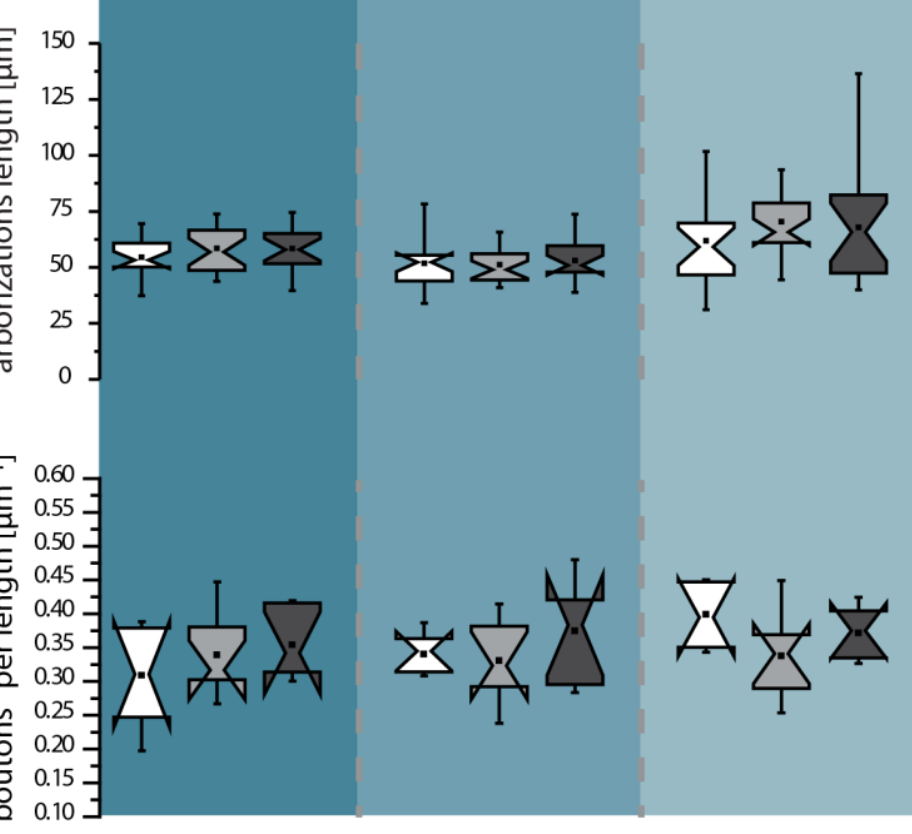

$\frac{1}{3} \sum_{i}^{1} \frac{1}{2}$
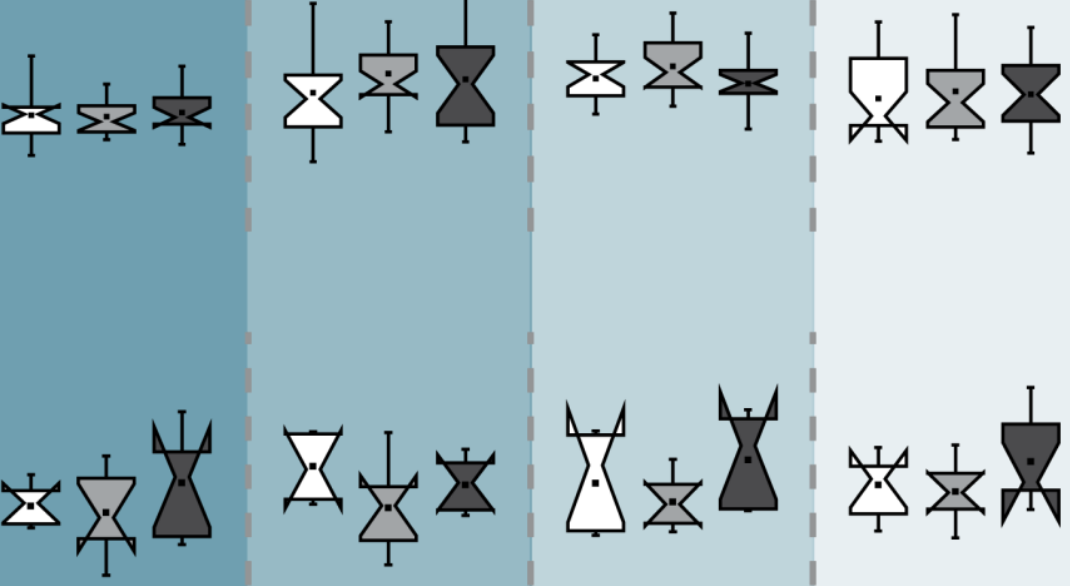

Figure 3.26. Effect of different calorie diets on $\mathbf{a} / \boldsymbol{\beta}$ cell morphology. Continued on next page. 
compartments, and the number of nodes within $a / \beta$ lobe compartments (figure $3.26 \mathrm{H}-$ J)

In sum, the morphologies of $\mathrm{Y}, \mathrm{a}^{\prime} / \beta^{\prime}$, and $a / \beta$ neurons were hardly affected by keeping the flies the cells derived from on different calorie diets for about one week during adult life. Thus, conditions of under-, or oversupply mainly concern the MB extrinsic network but not the MB intrinsic KCs.

Figure 3.26. Effect of different calorie diets on $\alpha / \beta$ cell morphology.

Flies expressing hexameric mCherry in individual $a / \beta$ Kenyon cells via the Gal4 driver OK107 were kept on different calorie diets for 7-8 days during adulthood. Single cells were compared statistically dependent on the flies' diet and in regard to $a / \beta$ cell morphological parameters. From the 19 compared parameters no significant differences were detected between any of the groups. A) Illustration of $a \mathrm{a} / \beta$ cell and its arborizations within different parts of the mushroom body that were quantified for the statistical analysis of different food diets and their effect on $a / \beta$ Kenyon cell morphology.

$B, C)$ The quantified parameters are highlighted in confocal images of the mushroom body calyx (B) and the mushroom body lobes (C).

$D-G) a / \beta$ Kenyon cells deriving from animals kept on different calorie diets during imago stage were compared statistically in regard to their morphological characteristics within the cells' input and output regions. No significant differences between $a / \beta$ cells deriving from flies kept on different calorie diets were detected in the number of dendritic claws (D), the dendrite length $(E)$, the axon length $(F)$, and the number of axon nodes $(G)$.

$\mathrm{H}-\mathrm{J}$ ) a/ $\beta$ Kenyon cells deriving from animals kept on different calorie diets during imago stage were compared statistically in regard to their compartment-specific arborizations. No significant differences were detected regarding the number of nodes within a1-3 and $\beta 1-2$, the arborizations length within $a 1-3$ and $\beta 1-2$, and the "bouton"-density within $a 1-3$ and $\beta 1-2$ between $a / \beta$ cells deriving from flies kept on different calorie diets.

Data were compared statistically using ANOVA if data were normally distributed or a KruskalWallis test if date were not normally distributed. Normality of the distributions was determined by the Shapiro-Wilk test. p-values were corrected for multiple comparisons according to Bonferroni's principle. 


\section{Discussion}

\subsection{Single y lobe Kenyon cells are morphologically diverse}

It is of general interest to understand how behavior is mediated by neural circuits and how single units contribute to the integration process between sensory input to behavioral output (Luo et al., 2008). One popular model system, the Drosophila MBs, consisting of $\sim 2,000 \mathrm{KCs}$ each, have been studied in detail (Aso et al., 2014a; Fiala, 2007; Masse et al., 2009). Seven KC subtypes have been described that differ in terms of gene expression, birth order, axonal arborizations patterns, and their functional role in different behaviors (Aso et al., 2009, 2014a, 2014b; Krashes et al., 2007; Lee et al., 1999; Lin et al., 2007; Perisse et al., 2013a, 2013b; Tanaka et al., 2008; and figures $1.4,1.6)$. KCs of the $y$ main subtype constitute the largest $\mathrm{KC}$ subset in respect to volume and cell number (Aso et al., 2009, 2014a). Functional analyses have revealed that $\mathrm{Y}$ main $\mathrm{KCs}$ play a role in olfactory associative appetitive, and aversive short- and long-term memory formation and retrieval (Blum et al., 2009; CervantesSandoval et al., 2013; Das et al., 2014; Dubnau et al., 2001; Pascual and Preat, 2001; Scheunemann et al., 2012; Zars, 2000), motivational control of thirst (Lin et al., 2014b) and hunger (Huetteroth et al., 2015; Perisse et al., 2016), courtship conditioning (Manoli et al., 2005), and sleep regulation (Aso et al., 2014b; Joiner et al., 2006; Pitman et al., 2006; Sitaraman et al., 2015).

Here, the morphological diversity of $\mathrm{Y}$ main KCs was studied in order to understand how single cells contribute to functional diversity of the cell population. $\mathrm{Y}$ main KCs have so far not been subdivided into functional or morphological subclasses. In the present study, it was found that $\mathrm{Y}$ main KCs exhibit a large variety of different sizes and morphologies. The set of $75 \mathrm{Y}$ main $\mathrm{KCs}$ was compared statistically in respect to parameters that describe gross cell morphology, and parameters that describe compartment specific innervations in the MB lobes. The compartments are of special interest because they have been show to constitute rather independent computational units for multimodal signal integration (Aso and Rubin, 2016; Aso et al., 2014a, 2014b; Hige et al., 2015a; Owald and Waddell, 2015). In each of the quantified parameters $Y$ 
main KCs exhibit a vast range of sizes. For example, the axon length ranged between $129.9 \mu \mathrm{m}$ and $407.2 \mu \mathrm{m}$, and the claw number between 2 and 10 (see table 3.1 and figures 3.7-3.10). Similarly, within compartment arborizations varied strongly, as for example in $\gamma 2$ from $28 \mu \mathrm{m}$ to $127.4 \mu \mathrm{m}$, and in $\gamma 5$ from $0 \mu \mathrm{m}$ to $105.3 \mu \mathrm{m}$. Former studies confirm the observed variability in KC morphometrics: the number of dendritic claws was reported to lie between 4 and 12 (Gruntman and Turner, 2013), or 0 and 12 (Butcher et al., 2012); the number of axonal branches between 3 and 17 (here between 6 and 29); the cumulative axon branch length between $\sim 20$ and $120 \mu \mathrm{m}$ (Tessier and Broadie, 2008). In sum, it was shown for the first time that $\mathrm{Y}$ main $\mathrm{KCs}$, a cell type required for various behaviors including learning and memory, is composed of cells that are not uniform in their morphology, but exhibit a large variety of different shapes and sizes.

Heterogeneity of neurons is a problem also encountered in the analysis of vertebrate nervous systems, especially since Golgi staining was introduced to label single cells (reviewed in Agnati et al., 2007). Nowadays, cell types are typically identified by a combination of methods including the quantification of morphological, molecular, and physiological features (Petilla Interneuron Nomenclature Group et al., 2008). Are KCs similarly diverse in their physiological and biochemical properties as they are morphologically? Due to the small size of invertebrate neurons, KC soma diameter sizes lie around 4-5 $\mu \mathrm{m}$ (data not shown), electrophysiological recordings are rare. In a few studies though, KC patch-clamp recordings were performed, showing that KCs have very low baseline firing rates. Baseline firing rates in $a / \beta$ and $Y K C s$ were essentially absent, whereas $a^{\prime} / \beta^{\prime}$ showed stable activity around 0.2 spikes per second. In fact, out of the 71 cells measured in one study, 14 did not show any spontaneous activity at all, even though current injections verified their spiking ability (Turner et al., 2008). Gruntman and Turner (2013) did not distinguish between KC types, however, they found that most of the cells they measured, and were shown to be functional, did not have any baseline firing rate. However, it is not clear to which extend soma recordings reflect axonal activity (Gouwens and Wilson, 2009).

As described in section 1.4, KCs are sparse not only in spontaneous activity but also in terms of odor responsiveness. Independent of cell type though, some individual KCs were seen that responded broadly to a wide range of odors, while others showed highly specialized responses (Honegger et al., 2011; Turner et al., 2008). In average, KCs responded to $6 \%$ of odors presented, while response likelihood increased from $\mathrm{Y} K \mathrm{KCs}$ to $a / \beta$ KCs to $a^{\prime} / \beta^{\prime}$ KCs (Turner et al., 2008). It has not been resolved, whether or not the observed differences are due to intrinsic properties of the cells or due to KC-PN 
connectivity by dendritic claws, since the number of odor responsive claws were shown to correlate with spiking probability after PN stimulation (Gruntman and Turner, 2013), and the average number of claws is different between KC types (Butcher et al., 2012; and figures 3.24-3.26). Moreover, as mentioned above, $\mathrm{KC}$ soma voltage at axonal spiking might only partly be correlated, as it is the case for PNs (Gouwens and Wilson, 2009).

Differences in molecular features of neurons like the expression of transcription factors, neurotransmitters, neuropeptides, transporters, cell surface proteins, ion channels, receptors, and many more, have been used to classify vertebrate neurons (Petilla Interneuron Nomenclature Group et al., 2008). In Drosophila, initial studies have used the enhancer trap technique (Wilson et al., 1989) to target specific KC subtypes that are hence distinct in promoter activity patterns (e.g. Aso et al., 2009; Yang et al., 1995) or are different in respect to antigen expression (Crittenden et al., 1998). Moreover, different proteins have been shown to be required in distinct $K C$ types, like the rutabaga encoded adenylyl cyclase in $a / \beta$ and $y$ KCs (Blum et al., 2009; Scheunemann et al., 2012), Neurofibramatosis 1 in only a/ $\beta$ KCs (Buchanan and Davis, 2010; Guo et al., 2000), and the D1-like dopamine receptor DopR in y cells (Qin et al., 2012), just to name a few (for review see Kahsai and Zars, 2011; Margulies et al., 2005). In a comprehensive analysis of KC gene expression, the genomic enhancer fragments located upstream of genes encoding neuron specific promoters have been cloned in order to drive Gal4 expression in transgenic flies. Using the split-Gal4 system (Luan et al., 2006) it was possible to identify cell types that overlap in the activity of different promoters. This approach led to the definition of all known KC subtypes (Aso et al., 2014a; Jenett et al., 2012). However, cell types are certainly defined by the expression of more than two genes. Crocker et al (2016) employed single cell type RNA sequencing of cells harvested via patch pipets to examine the transcriptome of KCs. Thus, they were able to show distinct expression profiles of $a / \beta$ and $y \mathrm{KCs}\left(a^{\prime} / \beta^{\prime}\right.$ were not analyzed) in regard to neurotransmitter receptors, peptides, and peptide receptors among others. Nevertheless, a comprehensive analysis at the single cell level to judge individual diversity of gene expression has not been performed so far.

Furthermore, the architecture of calycal microglomeruli has been described in detail and was shown to be of a complex and molecular diverse organization (Butcher et al., 2012; Leiss et al., 2009; Yasuyama et al., 2002). Various elements contribute to the polymorphic shapes of calycal microglomeruli, which are composed of one projection neuron bouton and its postsynaptic partners (Yasuyama et al., 2002). Projection neurons were shown in electron mircrographs to contain clear core vesicles as well as 
dense core vesicles. Thus, KCs likely receive not only cholinergic but also peptidergic input from PNs (Butcher et al., 2012; Yasuyama et al., 2002). Furthermore, it was shown that dense core vesicle containing PN boutons are smaller in size than other PN boutons (Butcher et al., 2012). In general, presynaptic terminals in projection neuron boutons are widely distributed, and were classified as T-bar ribbon and non-ribbon synapses that occur in equal measure (Leiss et al., 2009). The organization of T-bar ribbon synapses adds another layer of complexity that shall not be discussed here and has been described elsewhere (Sigrist and Schmitz, 2011; Wichmann and Sigrist, 2010). The number of synapses in presynaptic PN boutons range between 24-69, which are each opposed by an average number of 4.5-6.3 postsynaptic densities in KCs depending on the type of PN (Butcher et al., 2012). Other components of the microglomerular network are GABAergic neurites, contributed by the anterior paired lateral neuron (Tanaka et al., 2008), serotoninergic neurites, contributed by the dorsal paired medial neuron (Waddell et al., 2000), SIFamidergic neurites (Tanaka et al., 2008; Verleyen et al., 2004), dopaminergic neurites from the PPL2ab cluster (Mao and Davis, 2009), and octopaminergic neurites (Busch et al., 2009). In addition, KCs also provide output within the calyx area as was shown by antibody labeling of presynaptic terminals (Christiansen et al., 2011). For overview and discussion of the MB calyx network also see Aso et al (2014a) and Pech et al (2013b). In sum, the analysis of the calycal network of KCs and MB extrinsic neurons reveals a complex structure that has the potential to strongly influence the excitability of individual KCs. If there is a pattern of connectivity between KCs and other cells of the network or if KCs are randomly connected to extrinsic neurons is under debate and has not even been answered for PN-KC connections (Caron et al., 2013; Gruntman and Turner, 2013).

To sum up, single cell data of KCs as they were acquired in the present study for morphological parameters are not available in such details for other cellular characteristics like physiology and biochemistry. It will be interesting, to find out about how the described morphological diversity of single $\mathrm{Y}$ KCs is related to other cellular parameters in order to get a complete picture of the cells' identity. In fact, it has been achieved to measure $\mathrm{Ca}^{2+}$ fluxes of single $\mathrm{y}$ cells that will be related to the cells' morphology (F. Bilz, personal communication). This approach gives the opportunity to investigate how morphology and physiology interact to facilitate the hypothesized functional plasticity of single cells, which are required for learning and memory. 


\subsection{Description of Kenyon cell subtypes}

\subsection{1 y Kenyon cell subtypes can be defined by axonal bifurcation}

KCs can, furthermore, be described from a qualitative perspective. Here, it was found that $\mathrm{Y}$ main KCs can be separated into four groups based on axonal bifurcations in the $y$ lobes. Four different $y$ cell classes were identified that have the primary node in $y 2$, $\gamma^{3}$, or $\gamma 4$, or are not bifurcating. Classically, $a^{\prime} / \beta^{\prime}$ and $a / \beta K C$ s are considered the only bifurcating KC types. They extend one vertical branch into the $a / a^{\prime}$ lobes and one horizontal branch into the $\beta / \beta^{\prime}$ lobes (Crittenden et al., 1998). According to a previous report, there is one type of adult $\mathrm{Y}$ KCs that extend a side branch into the vertical lobes (Strausfeld et al., 2003). Those cells, however, were not found here. It seems likely, that they are remnants of larval $\mathrm{Y}$ KCs, which escaped restructuring during pupal stage. Though, in this study, one $\mathrm{Y} \mathrm{KC}$ was found that does not extend through the complete $\mathrm{Y}$ lobe, but terminates in the $\mathrm{Y} 2$ compartment. Probably, this cell is also not a representative of another $\mathrm{Y}$ cell type, but rather constitutes an individual case of a cell that failed proper outgrowth after pruning, because no more cells of this type were found. In the present study, the exact topography of bifurcating $y$ cell branches was not determined. Future research has to show if branches from bifurcating $y$ cells occupy specific, three dimensionally defined, regions of the $y$ lobes. It is known of the $Y$ dorsal subset of the $Y$ lobe that they arborize in specific parts of the $Y$ lobe compartments, most prominently may be in the dorsal tip of the $\gamma 5$ compartment (Aso et al., 2014a; and figure 1.4).

Compartments of the $a / \beta$ and the $a^{\prime} / \beta^{\prime} K C s$ are defined by the innervation patterns of $M B$ extrinsic dopaminergic and MB output neurons, which were found to sometimes target $K C$ subclasses like the $a^{\prime} / \beta^{\prime}$ anterior-posterior, and the $a^{\prime} / \beta^{\prime}$ middle neurons, and the $\alpha / \beta$ posterior, surface, and core neurons. However, cell type specific innervations by MB extrinsic neurons are, for the $y$ lobe, not even observed in the case of the $Y$ dorsal subset (Aso et al., 2014a). Thus, if there are $\mathrm{Y}$ main KC arborizations specific to subcompartmental structures in the $y$ lobe, those fibers might interact not with dopaminergic neurons, but with other neuron types. $\mathrm{Y}$ KCs were found to be in close proximity to octopaminergic/tyraminergic, and serotoninergic neurons (Pech et al., 2013b), and various peptide receptors, including the pdf receptor, the SIFamide receptor, and insulin receptor, as well as receptors for biogenic amines, including the octopamine OAMB and serotonin $5 \mathrm{HT} 1 \mathrm{~A}$, and $5 \mathrm{HT} 1 \mathrm{~B}$ receptors are expressed in $\mathrm{Y} \mathrm{KCs}$ (Crocker et al., 2016), indicating the presence of more synaptic partners that give 
input to y KCs. Additionally, NMDA receptors, shown to be necessary for olfactory longterm memory formation (Xia et al., 2005), are expressed in Y KCs (Crocker et al., 2016). Conclusively, if the here described anatomical y KC classes have a functional role in the MB network, this role has to be proven in future studies.

\subsection{2 y Kenyon cell subtypes are identified by hierarchical cluster analysis}

The present study provides the first comprehensive morphological analysis of KCs at the single cell level. Thus, it was possible to describe novel Y KC subtypes using cluster analysis. Hierarchical clustering using Ward's method (Murtagh and Legendre, 2014; Ward, 1963) as a criterion for cluster fusion has been proven in several studies to successfully find clusters of test data sets (Blashfield, 1976; Hands and Everitt, 1987), or to identify cell types of vertebrate and invertebrate nervous systems (Cauli et al., 2000; Jefferis et al., 2007; Karagiannis et al., 2009; Murtagh and Contreras, 2012; Wong et al., 2002). In the present study, the same method was used in order to find types of KCs based on morphological data. Two cell types that are each composed of two subtypes were found when analyzing gross $\mathrm{Y}$ main $\mathrm{KC}$ morphology. The two types can be described as one type consisting of 'big' cells, and the other of 'small' cells. In respect to all four input parameters the 'big' cluster cells exhibit significantly higher values than the 'small' cluster cells. Furthermore, it was found in a second cluster analysis that $\mathrm{KCs}$ form two cluster regarding their axonal arborizations within $\mathrm{Y}$ compartments $1-5$. The two clusters are distinguished by their arborizations within $y 2-$ 4. One type of cells had significantly longer arbors in all of the three mentioned compartments. The 'big' and 'small' clusters of the first analysis are not correlated to the compartment clusters of the second analysis.

It has become increasingly common to generate anatomical standard brain models that can be used to map individual cells deriving from different animals onto one template (e.g. Aso et al., 2014a; Chiang et al., 2011; Jefferis et al., 2007; Lin et al., 2007; Peng et al., 2011). This method has been applied to analyze a set of 1,664 KCs out of the FlyCircuit data base (Chiang et al., 2011) in regard to their three dimensional arborizations pattern. Using hierarchical clustering it was possible to determine all known KC subtypes as well as two novel $\mathrm{Y}$ KC subtypes that are a subset of $\mathrm{Y}$ main (Costa et al., 2016). However, the novel types were not described in detail, so that it remains to be shown how they are morphologically different. 
As mentioned before cell type identification usually relies on the inclusion of not only morphological but also physiological and molecular cell features (Petilla Interneuron Nomenclature Group et al., 2008). Since KCs are not sufficiently studied in respect to those features, KC subtype classification until now has to depend on morphological characteristics of KCs.

\subsection{Nutritional value and structural plasticity of Kenyon cells}

Although, there is a vast literature background about the Drosophila MBs and their role in associative learning and memory, studies on structural plasticity of KCs, the cells that supposedly carry the memory, are scarce. Here, structural plasticity of KCs was investigated by exposing flies with labeled single KCs to different environmental conditions. The kind of conditions chosen have been shown to strongly shape the innervation pattern of dopaminergic and $M B$ output neurons in the MB lobes in $a$ compartment specific manner ( $T$. Riemensperger, personal communication). Therefore, adult flies were kept on food with different caloric values for 7-8 days. The morphologies of $K C s$ of the $Y, a^{\prime} / \beta^{\prime}$, and $a / \beta$ type deriving from flies raised on these food conditions were compared statistically. However, no major changes in $\mathrm{KC}$ morphologies could be observed, although more axonal nodes in the $\mathrm{y} 5$ compartment of flies raised on medium calorie food than in flies raised on low or high calorie food, were found. Additionally, the dendrites of $a^{\prime} / \beta^{\prime}$ cells were longer of flies kept on medium calorie food than of flies kept on high calorie food, and the "bouton" density in the $\beta^{\prime} 2$ compartment was higher in KCs of flies kept on low calorie food than of flies kept on medium calorie food. Overall though, a clear pattern of neural structural changes as a consequence of differences in diet could not be detected. There are several possible explanations for the absence of such a plasticity. (1) Changes are too marginal to be detected by the number of comparisons in this study. (2) KC structural changes happen on a smaller scale, e.g. in the molecular architecture of synapses. (3) Network adaptation to food conditions is achieved mainly by changes in MB extrinsic neurons.

According to (1) the sample size of the acquired neurons is too low to show structural effects because between neuron variability is high. This is true, as can be seen by the range of sizes of individual cells, however a normalized measure, the "bouton" density, was taken to control for size-differences of KCs. The observation that honey bee KC dendrite length was correlated with foraging experience (Farris et al., 2001), could not be shown here for Drosophila. However, foraging experience of honey bees is far more 
complex than just food intake, but includes a range of behavioral skills as, for example, navigation and food source identification (Robinson, 1992). As for (2), evidences of small scale changes in synaptic architecture and physiology are numerous across phyla (reviewed for example in Mayford et al., 2012). In Drosophila, changes in synaptic marker expression were found for example in sleep deprived flies (Gilestro et al., 2009), and ultrastructural elaborations of presynaptic active zones were observed in aged flies (Gupta et al., 2016). Genes relevant for long-term memory formation and synaptic remodeling have been shown to be especially required in KCs (e.g. Akalal et al., 2010). It seems likely that according to (3) MB extrinsic networks respond stronger in terms of structural plasticity to environmental conditions. First, because of the findings in GRASP signal changes by T. Riemensperger, and second, because KCs have been found to be relatively resistant to structural changes even by developmental overexpression of memory affecting DISC1 (Furukubo-Tokunaga et al., 2016), extended periods of ChR2 induced activity (Tessier and Broadie, 2008), or sleep deprivation (Bushey et al., 2011).

\subsection{Outlook}

In the present study, a population of single y KCs was described morphologically and classified using several approaches. Although it is agreed upon the role of KCs in learning and memory, direct evidence of plasticity occurring in KCs is still missing. The analysis of structural plasticity of single KCs deriving from all major KC classes has revealed only slight changes happening in KCs in response to different feeding conditions. Thus, approaches that target the functional anatomy of individual KCs will be promising. Until now, most studies focused on $\mathrm{KC}$ population responses (reviewed in Guven-Ozkan and Davis, 2014). Novel $\mathrm{Ca}^{2+}$ imaging techniques have enabled researchers to observe odor evoked activity in single KCs at the calyx level (Gruntman and Turner, 2013). Single KCs' physiological responses and learning-induced functional plasticity of single KCs are currently under investigation (F. Bilz, personal communication). This intriguingly simple approach offers the possibility to not only correlate function and morphology of single KCs, but also to resolve the question of how individual KCs store and retrieve associative memory. 


\section{Summary}

Learning and memory is an adaptive behavioral trait that allows animals to make predictions about their environment based on previous experiences. Fruit flies that commonly use olfactory cues to locate food sources or mating partners, are able to assign value to odors after classical conditioning. The trace of the olfactory associative memory could successfully be localized to the Drosophila mushroom bodies, a central arthropod brain structure. Here, the mushroom body intrinsic Kenyon cells are believed to store the memory in the synaptic connection to mushroom body output neurons. So far, seven different Kenyon cell types have been described that can be distinguished based on gene expression patterns, reactivity to antibodies, birth order, and morphology. Furthermore, different Kenyon cell types have been implicated in distinct functional tasks. However, most of the studies were focused on populations of Kenyon cells. A comprehensive analysis of single Kenyon cells has not been performed.

In the present study, the morphology of single Kenyon cells of the $\mathrm{y}$ type was compared quantitatively in order to describe the variability of individual cell shapes, and to establish a basis for functional analyses. To this end, a set of 75 single $y$ Kenyon cells was collected using mosaic analysis with a repressible cell marker. The cell structures were digitalized by the manual skeleton tracing function implemented in the commercial software Neurolucida. To compare cell morphologies, four parameters were chosen. Two that describe the input region of the mushroom bodies, i.e. the dendrite length and the number of dendritic claws, and two that describe the output region, i.e. the axon length and the number of axon nodes. Furthermore, Kenyon cell arborizations within the output region, the mushroom body lobes, were analyzed in respect to the five $\mathrm{y}$ lobe compartments, which are defined by the innervations of dopaminergic and mushroom body output neurons.

It was found that $y$ Kenyon cells exhibit a vast range of morphologies, as seen by the variability in each of the quantified parameters. To find patterns within the set of cells, qualitative and quantitative approaches were taken. First, bifurcations of $y$ cells within the $y$ lobes were identified and used to group $Y$-type Kenyon cells into four classes. $Y$ cells are normally considered to be non-bifurcating cells, and future research has to show if bifurcation and functionality are correlated. Second, the quantified parameters 
were used as input variables for hierarchical clustering. Thus, several cell types were identified that rely on combinations of either the parameters, which describe input to, and output from the mushroom bodies, or the innervations of $Y$ Kenyon cells within $Y$ compartments $1-5$.

The role of Kenyon cells in learning and memory makes it likely that Kenyon cells are able to change in shape or functionality as a result of experience. Extrinsic conditions such as food supply and the social environment were shown to affect mushroom body volume and the innervations of mushroom body extrinsic neurons. Indeed, raising flies on different calorie food strongly affected Kenyon cell contacts to extrinsic neurons within specific compartments of the $\gamma, a^{\prime} / \beta^{\prime}$, and $a / \beta$ lobes. Thus, in the present study flies were exposed to the same calorie food conditions at imago stage and the cell morphologies of $Y, a^{\prime} / \beta^{\prime}$, and $a / \beta$ cells were compared quantitatively. However, in none of the cell types major morphological changes were observed. It seems likely that in the case of food intake mushroom body extrinsic neurons undergo adaptive changes, while Kenyon cells do not change morphologically. Changes in the physiology of Kenyon cells though, cannot be excluded. 


\section{Acknowledgements}

At first, I have to thank my supervisor Prof. Fiala for his constant support, and his advice regarding the design, implementation, and analysis of experiments. Prof. Fiala's deep knowledge of many research fields helped me to overcome all the difficult parts of the project, and Prof. Fiala always offered to help solving the different problems.

I further thank my thesis committee members Prof. Martin Göpfert and Dr. Camin Dean for support and helpful discussions during the thesis committee meetings and Martin Göpfert for reviewing the thesis.

Additionally, I am much obliged to Dr. T. Riemensperger, who gave valuable advice and supervision for the implementation of many experiments, and for his comments on the early manuscript of the thesis.

I thank the Göttingen Graduate School for Neurosciences, Biophysics, and Molecular Biosciences (GGNB) for funding, and for the organization of methods and skill courses.

I am also very thankful to Prof. Jochen Staiger, who provided the Neurolucida setup for cell reconstructions. Additionally, I thank Prof. Jochen Staiger and his PhD student Alvar Prönneke for advice and help for the application of Neurolucida.

Furthermore, I thank Dr. Bart Geurten for his help regarding cluster analyses, Florian Bilz for guidance on how to use Adobe Illustrator and to design figures, Shubham Dipt for very useful excel makros for data analyses, Atefeh Pooryasin for her setup, Carlotta Martelli for organizing the pizza seminar series, Hendrik Urbanke for providing help with behavioral experiments, and Dr. Heribert Gras, and Prof. Andreas Stumpner for solving problems with the confocal microscope.

Special thanks go to Jutta Böker. Without her, work in the lab would not have been possible! In addition, I am grateful to all other Fiala lab members for revising my experiments, helpful discussions, and for providing a very nice and stimulating working atmosphere: Atefeh Pooryasin, Büşra Çoban, Carlotta Martelli, Florian Bilz, Jonas Barth, Nicole Dörjer, Priyanka Singh, Sandra Schubert, Shubham Dipt, Simon Kobbenbring, Thomas Riemensperger, and Ulrike Pech. Also, I like to thank all members of the Göpfert lab for being such nice neighbors.

Finally, and most of all, I want to thank Atefeh, Uli, Shubham, Simon, Eva, Florian, and Lena for their friendship and company during the hard and the pleasant times of the PhD! 


\section{References}

Agnati, L.F., Genedani, S., Leo, G., Rivera, A., Guidolin, D., and Fuxe, K. (2007). One century of progress in neuroscience founded on Golgi and Cajal's outstanding experimental and theoretical contributions. Brain Res. Rev. 55, 167-189.

Akalal, D.-B.G., Yu, D., and Davis, R.L. (2010). A late-phase, long-term memory trace forms in the $Y$ neurons of Drosophila mushroom bodies after olfactory classical conditioning. J. Neurosci. 30, 16699-16708.

Akalal, D.-B.G., Yu, D., and Davis, R.L. (2011). The long-term memory trace formed in the Drosophila $\alpha / \beta$ mushroom body neurons is abolished in long-term memory mutants. J. Neurosci. 31, 5643-5647.

Akerboom, J., Chen, T.-W., Wardill, T.J., Tian, L., Marvin, J.S., Mutlu, S., Calderón, N.C., Esposti, F., Borghuis, B.G., Sun, X.R., et al. (2012). Optimization of a GCaMP calcium indicator for neural activity imaging. J. Neurosci. 32, 13819-13840.

Anderson, D.J., and Adolphs, R. (2014). A framework for studying emotions across species. Cell 157, 187-200.

Aristotle, (Translation by Ross, W.D., and Smith, J.A. (1910). The works of Aristotle (Oxford: Clarendon Press)).

Ashburner, M., and Bonner, J.J. (1979). The induction of gene activity in Drosophilia by heat shock. Cell 17, 241-254.

Aso, Y., and Rubin, G.M. (2016). Dopaminergic neurons write and update memories with cell-type-specific rules. eLife 5, e16135

Aso, Y., Grübel, K., Busch, S., Friedrich, A.B., Siwanowicz, I., and Tanimoto, H. (2009). The mushroom body of adult Drosophila characterized by GAL4 drivers. J. Neurogenet. 23, 156-172.

Aso, Y., Siwanowicz, I., Bräcker, L., Ito, K., Kitamoto, T., and Tanimoto, H. (2010). Specific dopaminergic neurons for the formation of labile aversive memory. Curr. Biol. 20, 1445-1451.

Aso, Y., Herb, A., Ogueta, M., Siwanowicz, I., Templier, T., Friedrich, A.B., Ito, K., Scholz, H., and Tanimoto, H. (2012). Three dopamine pathways induce aversive odor memories with different stability. PLoS Genet. 8, e1002768.

Aso, Y., Hattori, D., Yu, Y., Johnston, R.M., Iyer, N.A., Ngo, T.-T., Dionne, H., Abbott, L.F., Axel, R., Tanimoto, H., et al. (2014a). The neuronal architecture of the mushroom body provides a logic for associative learning. eLife 3, e04577.

Aso, Y., Sitaraman, D., Ichinose, T., Kaun, K.R., Vogt, K., Belliart-Guérin, G., Plaçais, P.-Y., Robie, A.A., Yamagata, N., Schnaitmann, C., et al. (2014b). Mushroom body 
output neurons encode valence and guide memory-based action selection in Drosophila. eLife 3, e04580.

Bailey, C.H., and Chen, M. (1989). Structural plasticity at identified synapses during long-term memory in Aplysia. J. Neurobiol. 20, 356-372.

Bailey, C.H., and Kandel, E.R. (1993). Structural changes accompanying memory storage. Annu. Rev. Physiol. 55, 397-426.

Bailey, C.H., and Kandel, E.R. (2008). Synaptic remodeling, synaptic growth and the storage of long-term memory in Aplysia. Prog. Brain Res. 169, 179-198.

Barnstedt, O., Owald, D., Felsenberg, J., Brain, R., Moszynski, J.-P., Talbot, C.B., Perrat, P.N., and Waddell, S. (2016). Memory-relevant mushroom body output synapses are cholinergic. Neuron $89,1237-1247$.

Barth, M., and Heisenberg, M. (1997). Vision affects mushroom bodies and central complex in Drosophila melanogaster. Learn. Mem. 4, 219-229.

Barth, J., Dipt, S., Pech, U., Hermann, M., Riemensperger, T., and Fiala, A. (2014). Differential associative training enhances olfactory acuity in Drosophila melanogaster. J. Neurosci. 34, 1819-1837.

Bell, J.S., and Wilson, R.I. (2016). Behavior reveals selective summation and max pooling among olfactory processing channels. Neuron 91, 425-438.

de Belle, J.S., and Heisenberg, M. (1994). Associative odor learning in Drosophila abolished by chemical ablation of mushroom bodies. Science 263, 692-695.

Blashfield, R.K. (1976). Mixture model tests of cluster analysis: Accuracy of four agglomerative hierarchical methods. Psychol. Bull. 83, 377-388.

van der Bliek, A.M., and Meyerowitz, E.M. (1991). Dynamin-like protein encoded by the Drosophila shibire gene associated with vesicular traffic. Nature 351,411-414.

Blum, A.L., Li, W., Cressy, M., and Dubnau, J. (2009). Short- and long-term memory in Drosophila require CAMP signaling in distinct neuron types. Curr. Biol. 19, 13411350.

Boto, T., Louis, T., Jindachomthong, K., Jalink, K., and Tomchik, S.M. (2014). Dopaminergic modulation of CAMP drives nonlinear plasticity across the Drosophila mushroom body lobes. Curr. Biol. 24, 822-831.

Bourne, J.N., and Harris, K.M. (2008). Balancing structure and function at hippocampal dendritic spines. Annu. Rev. Neurosci. 31, 47-67.

Brand, A.H., and Perrimon, N. (1993). Targeted gene expression as a means of altering cell fates and generating dominant phenotypes. Development 118, 401-415. 
Brigui, N., Le Bourg, E., and Médioni, J. (1990). Conditioned suppression of the proboscis-extension response in young, middle-aged, and old Drosophila melanogaster flies: acquisition and extinction. J. Comp. Psychol. 104, 289-296.

Brown, M.R., Crim, J.W., Arata, R.C., Cai, H.N., Chun, C., and Shen, P. (1999). Identification of a Drosophila brain-gut peptide related to the neuropeptide $Y$ family. Peptides 20, 1035-1042.

Buchanan, M.E., and Davis, R.L. (2010). A distinct set of Drosophila brain neurons required for neurofibromatosis type 1 -dependent learning and memory. J. Neurosci. 30, 10135-10143.

Buck, L., and Axel, R. (1991). A novel multigene family may encode odorant receptors: a molecular basis for odor recognition. Cell 65, 175-187.

Buckingham, H.W., and Finger, S. (1997). David Hartley's psychobiological associationism and the legacy of Aristotle. J. Hist. Neurosci. 6, 21-37.

Budnik, V. (1996). Synapse maturation and structural plasticity at Drosophila neuromuscular junctions. Curr. Opin. Neurobiol. 6, 858-867.

Burke, C.J., Huetteroth, W., Owald, D., Perisse, E., Krashes, M.J., Das, G., Gohl, D., Silies, M., Certel, S., and Waddell, S. (2012). Layered reward signalling through octopamine and dopamine in Drosophila. Nature 492, 433-437.

Busch, S., Selcho, M., Ito, K., and Tanimoto, H. (2009). A map of octopaminergic neurons in the Drosophila brain. J. Comp. Neurol. 513, 643-667.

Bushey, D., Tononi, G., and Cirelli, C. (2011). Sleep and synaptic homeostasis: structural evidence in Drosophila. Science 332, 1576-1581.

Butcher, N.J., Friedrich, A.B., Lu, Z., Tanimoto, H., and Meinertzhagen, I.A. (2012). Different classes of input and output neurons reveal new features in microglomeruli of the adult Drosophila mushroom body calyx. J. Comp. Neurol. 520, 2185-2201.

Byers, D., Davis, R.L., and Kiger, J.A. (1981). Defect in cyclic AMP phosphodiesterase due to the dunce mutation of learning in Drosophila melanogaster. Nature 289, 7981.

Cajal, S.R., and Sánchez, D. (1915). Contribución al conocimiento de los centros nerviosos de los insectos (Madrid: Imprenta de Hijos de Nicolás Moya).

Campbell, R.E., Tour, O., Palmer, A.E., Steinbach, P.A., Baird, G.S., Zacharias, D.A., and Tsien, R.Y. (2002). A monomeric red fluorescent protein. Proc. Natl. Acad. Sci. U. S. A. $99,7877-7882$.

Carew, T.J., Walters, E.T., and Kandel, E.R. (1981). Classical conditioning in a simple withdrawal reflex in Aplysia californica. J. Neurosci. 1, 1426-1437. 
Caron, S.J.C., Ruta, V., Abbott, L.F., and Axel, R. (2013). Random convergence of olfactory inputs in the Drosophila mushroom body. Nature 497, 113-117.

Caroni, P., Donato, F., and Muller, D. (2012). Structural plasticity upon learning: regulation and functions. Nat. Rev. Neurosci. 13, 478-490.

Cassenaer, S., and Laurent, G. (2007). Hebbian STDP in mushroom bodies facilitates the synchronous flow of olfactory information in locusts. Nature $448,709-713$.

Cassenaer, S., and Laurent, G. (2012). Conditional modulation of spike-timingdependent plasticity for olfactory learning. Nature 482, 47-52.

Cauli, B., Porter, J.T., Tsuzuki, K., Lambolez, B., Rossier, J., Quenet, B., and Audinat, E. (2000). Classification of fusiform neocortical interneurons based on unsupervised clustering. Proc. Natl. Acad. Sci. U. S. A. 97, 6144-6149.

Cervantes-Sandoval, I., and Davis, R.L. (2012). Distinct traces for appetitive versus aversive olfactory memories in DPM neurons of Drosophila. Curr. Biol. 22, 1247-1252.

Cervantes-Sandoval, I., Martin-Pena, A., Berry, J.A., and Davis, R.L. (2013). Systemlike consolidation of olfactory memories in Drosophila. J. Neurosci. 33, 9846-9854.

Chabaud, M.-A., Devaud, J.-M., Pham-Delègue, M.-H., Preat, T., and Kaiser, L. (2006). Olfactory conditioning of proboscis activity in Drosophila melanogaster. J. Comp. Physiol. A 192, 1335-1348.

Chen, C.-C., Wu, J.-K., Lin, H.-W., Pai, T.-P., Fu, T.-F., Wu, C.-L., Tully, T., and Chiang, A.-S. (2012). Visualizing long-term memory formation in two neurons of the Drosophila brain. Science 335, 678-685.

Chen, C.N., Denome, S., and Davis, R.L. (1986). Molecular analysis of cDNA clones and the corresponding genomic coding sequences of the Drosophila dunce gene, the structural gene for cAMP phosphodiesterase. Proc. Natl. Acad. Sci. U. S. A. 83, 93139317.

Cheung, U., Atwood, H.L., and Zucker, R.S. (2006). Presynaptic effectors contributing to cAMP-induced synaptic potentiation in Drosophila. J. Neurobiol. 66, 273-280.

Chiang, A.-S., Lin, C.-Y., Chuang, C.-C., Chang, H.-M., Hsieh, C.-H., Yeh, C.-W., Shih, C.-T., Wu, J.-J., Wang, G.-T., Chen, Y.-C., et al. (2011). Three-dimensional reconstruction of brain-wide wiring networks in Drosophila at single-cell resolution. Curr. Biol. 21, 1-11.

Choi, G.B., Stettler, D.D., Kallman, B.R., Bhaskar, S.T., Fleischmann, A., and Axel, R. (2011). Driving opposing behaviors with ensembles of piriform neurons. Cell 146, 1004-1015.

Christiansen, F., Zube, C., Andlauer, T.F.M., Wichmann, C., Fouquet, W., Owald, D., Mertel, S., Leiss, F., Tavosanis, G., Farca Luna, A.J., et al. (2011). Presynapses in 
Kenyon cell dendrites in the mushroom body calyx of Drosophila. J. Neurosci. 31, 9696-9707.

Claridge-Chang, A., Roorda, R.D., Vrontou, E., Sjulson, L., Li, H., Hirsh, J., and Miesenböck, G. (2009). Writing memories with light-addressable reinforcement circuitry. Cell 139, 405-415.

Cohn, R., Morantte, I., and Ruta, V. (2015). Coordinated and compartmentalized neuromodulation shapes sensory processing in Drosophila. Cell 163, 1742-1755.

Connolly, J.B., Roberts, I.J., Armstrong, J.D., Kaiser, K., Forte, M., Tully, T., and O'Kane, C.J. (1996). Associative learning disrupted by impaired Gs signaling in Drosophila mushroom bodies. Science 274, 2104-2107.

Connors, B.W., and Regehr, W.G. (1996). Neuronal firing: does function follow form? Curr. Biol. 6, 1560-1562.

Costa, M., Manton, J.D., Ostrovsky, A.D., Prohaska, S., and Jefferis, G.S.X.E. (2016). NBLAST: rapid, sensitive comparison of neuronal structure and construction of neuron family databases. Neuron 91, 293-311.

Couto, A., Alenius, M., and Dickson, B.J. (2005). Molecular, anatomical, and functional organization of the Drosophila olfactory system. Curr. Biol. 15, 1535-1547.

Crittenden, J.R., Skoulakis, E.M., Han, K.-A., Kalderon, D., and Davis, R.L. (1998). Tripartite mushroom body architecture revealed by antigenic markers. Learn. Mem. 5, 38-51.

Crocker, A., Guan, X.-J., Murphy, C.T., and Murthy, M. (2016). Cell-type-specific transcriptome analysis in the Drosophila mushroom body reveals memory-related changes in gene expression. Cell Rep. 15, 1580-1596.

Das, G., Klappenbach, M., Vrontou, E., Perisse, E., Clark, C.M., Burke, C.J., and Waddell, S. (2014). Drosophila learn opposing components of a compound food stimulus. Curr. Biol. 24, 1723-1730.

Dash, P.K., Hochner, B., and Kandel, E.R. (1990). Injection of the cAMP-responsive element into the nucleus of Aplysia sensory neurons blocks long-term facilitation. Nature 345, 718-721.

Datta, S.R., Vasconcelos, M.L., Ruta, V., Luo, S., Wong, A., Demir, E., Flores, J., Balonze, K., Dickson, B.J., and Axel, R. (2008). The Drosophila pheromone cVA activates a sexually dimorphic neural circuit. Nature 452, 473-477.

Davis, R.L. (1996). Physiology and biochemistry of Drosophila learning mutants. Physiol. Rev. 76, 299-317.

Davis, R.L. (2004). Olfactory learning. Neuron 44, 31-48.

Davis, R.L. (2011). Traces of Drosophila Memory. Neuron 70, 8-19. 
Davis, R.L., and Kiger, J.A. (1981). Dunce mutants of Drosophila melanogaster: mutants defective in the cyclic AMP phosphodiesterase enzyme system. J. Cell Biol. 90, 101-107.

De Bruyne, M., Foster, K., and Carlson, J.R. (2001). Odor coding in the Drosophila antenna. Neuron 30, 537-552.

Diegelmann, S., Nieratschker, V., Werner, U., Hoppe, J., Zars, T., and Buchner, E. (2006). The conserved protein kinase-A target motif in synapsin of Drosophila is effectively modified by pre-mRNA editing. BMC Neurosci. 7, 76.

Dill, M., Wolf, R., and Heisenberg, M. (1993). Visual pattern recognition in Drosophila involves retinotopic matching. Nature $365,751-753$.

Donlea, J.M., Ramanan, N., and Shaw, P.J. (2009). Use-dependent plasticity in clock neurons regulates sleep need in Drosophila. Science 324, 105-108.

Donlea, J.M., Ramanan, N., Silverman, N., and Shaw, P.J. (2014). Genetic rescue of functional senescence in synaptic and behavioral plasticity. Sleep 37, 1427-1437.

Drain, P., Folkers, E., and Quinn, W.G. (1991). cAMP-dependent protein kinase and the disruption of learning in transgenic flies. Neuron 6, 71-82.

Dubnau, J., Grady, L., Kitamoto, T., and Tully, T. (2001). Disruption of neurotransmission in Drosophila mushroom body blocks retrieval but not acquisition of memory. Nature $411,476-480$.

Dudai, Y., Jan, Y.N., Byers, D., Quinn, W.G., and Benzer, S. (1976). dunce, a mutant of Drosophila deficient in learning. Proc. Natl. Acad. Sci. U. S. A. 73, 1684-1688.

Dujardin, F. (1850). Mémoire sur le système nerveux des insectes. Ann. Sci. Nat. Zool. 14, 195-206.

Durst, C., Eichmüller, S., and Menzel, R. (1994). Development and experience lead to increased volume of subcompartments of the honeybee mushroom body. Behav. Neural Biol. 62, 259-263.

Ejima, A., Smith, B.P.C., Lucas, C., Levine, J.D., and Griffith, L.C. (2005). Sequential learning of pheromonal cues modulates memory consolidation in trainer-specific associative courtship conditioning. Curr. Biol. 15, 194-206.

Erber, J., Masuhr, T., and Menzel, R. (1980). Localization of short-term memory in the brain of the bee, Apis mellifera. Physiol. Entomol. 5, 343-358.

Fahrbach, S.E. (2006). Structure of the mushroom bodies of the insect brain. Annu. Rev. Entomol. 51, 209-232.

Farris, S.M., Robinson, G.E., and Fahrbach, S.E. (2001). Experience- and age-related outgrowth of intrinsic neurons in the mushroom bodies of the adult worker honeybee. J. Neurosci. 21, 6395-6404. 
Feinberg, E.H., Vanhoven, M.K., Bendesky, A., Wang, G., Fetter, R.D., Shen, K., and Bargmann, C.I. (2008). GFP Reconstitution Across Synaptic Partners (GRASP) defines cell contacts and synapses in living nervous systems. Neuron 57, 353-363.

Fiala, A. (2007). Olfaction and olfactory learning in Drosophila: recent progress. Curr. Opin. Neurobiol. 17, 720-726.

Fiala, A., Spall, T., Diegelmann, S., Eisermann, B., Sachse, S., Devaud, J.-M., Buchner, E., and Galizia, C.G. (2002). Genetically expressed cameleon in Drosophila melanogaster is used to visualize olfactory information in projection neurons. Curr. Biol. 12, 1877-1884.

Fire, A., Xu, S., Montgomery, M.K., Kostas, S.A., Driver, S.E., and Mello, C.C. (1998). Potent and specific genetic interference by double-stranded RNA in Caenorhabditis elegans. Nature 391, 806-811.

Foster, J.L., Higgins, G.C., and Jackson, F.R. (1988). Cloning, sequence, and expression of the Drosophila cAMP-dependent protein kinase catalytic subunit gene. J. Biol. Chem. 263, 1676-1681.

Fouquet, W., Owald, D., Wichmann, C., Mertel, S., Depner, H., Dyba, M., Hallermann, S., Kittel, R.J., Eimer, S., and Sigrist, S.J. (2009). Maturation of active zone assembly by Drosophila Bruchpilot. J. Cell Biol. 186, 129-145.

Friggi-Grelin, F., Coulom, H., Meller, M., Gomez, D., Hirsh, J., and Birman, S. (2003). Targeted gene expression in Drosophila dopaminergic cells using regulatory sequences from tyrosine hydroxylase. J. Neurobiol. 54, 618-627.

Furukubo-Tokunaga, K., Kurita, K., Honjo, K., Pandey, H., Ando, T., Takayama, K., Arai, Y., Mochizuki, H., Ando, M., Kamiya, A., et al. (2016). DISC1 causes associative memory and neurodevelopmental defects in fruit flies. Mol. Psychiatry. 21, 1232-1243.

Galili, D.S., Dylla, K.V., Lüdke, A., Friedrich, A.B., Yamagata, N., Wong, J.Y.H., Ho, C.H., Szyszka, P., and Tanimoto, H. (2014). Converging circuits mediate temperature and shock aversive olfactory conditioning in Drosophila. Curr. Biol. 24, 1712-1722.

Gerber, B., Tanimoto, H., and Heisenberg, M. (2004). An engram found? Evaluating the evidence from fruit flies. Curr. Opin. Neurobiol. 14, 737-744.

Gervasi, N., Tchénio, P., and Preat, T. (2010). PKA dynamics in a Drosophila learning center: Coincidence detection by rutabaga adenylyl cyclase and spatial regulation by dunce phosphodiesterase. Neuron 65, 516-529.

Gilestro, G.F., Tononi, G., and Cirelli, C. (2009). Widespread changes in synaptic markers as a function of sleep and wakefulness in Drosophila. Science 324, 109-112.

Golic, K.G. (1991). Site-specific recombination between homologous chromosomes in Drosophila. Science 252, 958-961. 
Golic, K.G., and Lindquist, S. (1989). The FLP recombinase of yeast catalyzes sitespecific recombination in the Drosophila genome. Cell 59, 499-509.

Goodwin, S.F., Del Vecchio, M., Velinzon, K., Hogel, C., Russell, S.R., Tully, T., and Kaiser, K. (1997). Defective learning in mutants of the Drosophila gene for a regulatory subunit of cAMP-dependent protein kinase. J. Neurosci. 17, 8817-8827.

Gormezano, I., Schneiderman, N., Deaux, E., and Fuentes, I. (1962). Nictitating membrane: classical conditioning and extinction in the albino rabbit. Science 138, 3334.

Gouwens, N.W., and Wilson, R.I. (2009). Signal propagation in Drosophila central neurons. J. Neurosci. 29, 6239-6249.

Grabe, V., Strutz, A., Baschwitz, A., Hansson, B.S., and Sachse, S. (2015). Digital in vivo $3 \mathrm{D}$ atlas of the antennal lobe of Drosophila melanogaster. J. Comp. Neurol. 523, 530-544.

Grant, D.A., and Adams, J.K. (1944). "Alpha" conditioning in the eyelid. J. Exp. Psychol. 34, 136-142.

Greenough, W.T., and Bailey, C.H. (1988). The anatomy of a memory: convergence of results across a diversity of tests. Trends Neurosci. 11, 142-147.

Griffith, L.C., Verselis, L.M., Aitken, K.M., Kyriacou, C.P., Danho, W., and Greenspan, R.J. (1993). Inhibition of calcium/calmodulin-dependent protein kinase in Drosophila disrupts behavioral plasticity. Neuron 10, 501-509.

Gruntman, E., and Turner, G.C. (2013). Integration of the olfactory code across dendritic claws of single mushroom body neurons. Nat. Neurosci. 16, 1821-1829.

Guo, A., and Götz, K.G. (1997). Association of visual objects and olfactory cues in Drosophila. Learn. Mem. 4, 192-204.

Guo, H.F., Tong, J., Hannan, F., Luo, L., and Zhong, Y. (2000). A neurofibromatosis1 -regulated pathway is required for learning in Drosophila. Nature 403, 895-898.

Gupta, N., and Stopfer, M. (2012). Functional analysis of a higher olfactory center, the lateral horn. J. Neurosci. 32, 8138-8148.

Gupta, V.K., Pech, U., Bhukel, A., Fulterer, A., Ender, A., Mauermann, S.F., Andlauer, T.F.M., Antwi-Adjei, E., Beuschel, C., Thriene, K., et al. (2016). Spermidine suppresses age-associated memory impairment by preventing adverse increase of presynaptic active zone size and release. PLoS Biol. 14, e1002563.

Guven-Ozkan, T., and Davis, R.L. (2014). Functional neuroanatomy of Drosophila olfactory memory formation. Learn. Mem. 21, 519-526.

Hallem, E.A., and Carlson, J.R. (2006). Coding of odors by a receptor repertoire. Cell 125, 143-160. 
Hamada, F.N., Rosenzweig, M., Kang, K., Pulver, S.R., Ghezzi, A., Jegla, T.J., and Garrity, P.A. (2008). An internal thermal sensor controlling temperature preference in Drosophila. Nature 454, 217-220.

Hammer, M., and Menzel, R. (1995). Learning and memory in the honeybee. J. Neurosci. 15, 1617-1630.

Han, P.L., Levin, L.R., Reed, R.R., and Davis, R.L. (1992). Preferential expression of the Drosophila rutabaga gene in mushroom bodies, neural centers for learning in insects. Neuron 9, 619-627.

Hands, S., and Everitt, B. (1987). A monte carlo study of the recovery of cluster structure in binary data by hierarchical clustering techniques. Multivar. Behav. Res. $22,235-243$.

Harrison, D.A., and Perrimon, N. (1993). Simple and efficient generation of marked clones in Drosophila. Curr. Biol. 3, 424-433.

Haynes, P.R., Christmann, B.L., and Griffith, L.C. (2015). A single pair of neurons links sleep to memory consolidation in Drosophila melanogaster. eLife 4, e03868.

Heimbeck, G., Bugnon, V., Gendre, N., Keller, A., and Stocker, R.F. (2001). A central neural circuit for experience-independent olfactory and courtship behavior in Drosophila melanogaster. Proc. Natl. Acad. Sci. U. S. A. 98, 15336-15341.

Heisenberg, M. (1998). What do the mushroom bodies do for the insect brain? An introduction. Learn. Mem. 5, 1-10.

Heisenberg, M. (2003). Mushroom body memoir: from maps to models. Nat. Rev. Neurosci. 4, 266-275.

Heisenberg, M., Borst, A., Wagner, S., and Byers, D. (1985). Drosophila mushroom body mutants are deficient in olfactory learning. J. Neurogenet. 2, 1-30.

Heisenberg, M., Heusipp, M., and Wanke, C. (1995). Structural plasticity in the Drosophila brain. J. Neurosci. 15, 1951-1960.

Hige, T., Aso, Y., Rubin, G.M., and Turner, G.C. (2015a). Plasticity-driven individualization of olfactory coding in mushroom body output neurons. Nature 526, 258-262.

Hige, T., Aso, Y., Modi, M.N., Rubin, G.M., and Turner, G.C. (2015b). Heterosynaptic plasticity underlies aversive olfactory learning in Drosophila. Neuron 88, 985-998.

Honegger, K.S., Campbell, R.A.A., and Turner, G.C. (2011). Cellular-resolution population imaging reveals robust sparse coding in the Drosophila mushroom body. $\mathrm{J}$. Neurosci. 31, 11772-11785. 
Huetteroth, W., Perisse, E., Lin, S., Klappenbach, M., Burke, C., and Waddell, S. (2015). Sweet taste and nutrient value subdivide rewarding dopaminergic neurons in Drosophila. Curr. Biol. 25, 751-758.

Ito, K., and Hotta, Y. (1992). Proliferation pattern of postembryonic neuroblasts in the brain of Drosophila melanogaster. Dev. Biol. 149, 134-148.

Ito, K., Awano, W., Suzuki, K., Hiromi, Y., and Yamamoto, D. (1997). The Drosophila mushroom body is a quadruple structure of clonal units each of which contains a virtually identical set of neurones and glial cells. Development 124, 761-771.

Ito, K., Suzuki, K., Estes, P., Ramaswami, M., Yamamoto, D., and Strausfeld, N.J. (1998). The organization of extrinsic neurons and their implications in the functional roles of the mushroom bodies in Drosophila melanogaster Meigen. Learn. Mem. 5, 5277.

Jain, A.K., Murty, M.N., and Flynn, P.J. (1999). Data clustering: a review. ACM Comput. Surv. 31, 264-323.

Jefferis, G.S., Marin, E.C., Stocker, R.F., and Luo, L. (2001). Target neuron prespecification in the olfactory map of Drosophila. Nature 414, 204-208.

Jefferis, G.S.X.E., Potter, C.J., Chan, A.M., Marin, E.C., Rohlfing, T., Maurer, C.R., and Luo, L. (2007). Comprehensive maps of Drosophila higher olfactory centers: spatially segregated fruit and pheromone representation. Cell 128, 1187-1203.

Jenett, A., Rubin, G.M., Ngo, T.-T.B., Shepherd, D., Murphy, C., Dionne, H., Pfeiffer, B.D., Cavallaro, A., Hall, D., Jeter, J., et al. (2012). A GAL4-driver line resource for Drosophila neurobiology. Cell Rep. 2, 991-1001.

Johard, H.A.D., Enell, L.E., Gustafsson, E., Trifilieff, P., Veenstra, J.A., and Nässel, D.R. (2008). Intrinsic neurons of Drosophila mushroom bodies express short neuropeptide F: Relations to extrinsic neurons expressing different neurotransmitters. J. Comp. Neurol. 507, 1479-1496.

Joiner, W.J., Crocker, A., White, B.H., and Sehgal, A. (2006). Sleep in Drosophila is regulated by adult mushroom bodies. Nature 441, 757-760.

Kahsai, L., and Zars, T. (2011). Learning and memory in Drosophila: behavior, genetics, and neural systems. Int. Rev. Neurobiol. 99, 139-167.

Kandel, E.R. (2001). The molecular biology of memory storage: a dialogue between genes and synapses. Science 294, 1030-1038.

Kandel, E.R., Dudai, Y., and Mayford, M.R. (2014). The molecular and systems biology of memory. Cell 157, 163-186. 
Karagiannis, A., Gallopin, T., Dávid, C., Battaglia, D., Geoffroy, H., Rossier, J., Hillman, E.M.C., Staiger, J.F., and Cauli, B. (2009). Classification of NPY-expressing neocortical interneurons. J. Neurosci. 29, 3642-3659.

Keene, A.C., Stratmann, M., Keller, A., Perrat, P.N., Vosshall, L.B., and Waddell, S. (2004). Diverse odor-conditioned memories require uniquely timed dorsal paired medial neuron output. Neuron 44, 521-533.

Keene, A.C., Krashes, M.J., Leung, B., Bernard, J.A., and Waddell, S. (2006). Drosophila dorsal paired medial neurons provide a general mechanism for memory consolidation. Curr. Biol. 16, 1524-1530.

Kenyon, F.C. (1896). The brain of the bee. A preliminary contribution to the morphology of the nervous system of the arthropoda. J. Comp. Neurol. 6, 133-210.

Kim, Y.-C., Lee, H.-G., and Han, K.-A. (2007). D1 dopamine receptor dDA1 is required in the mushroom body neurons for aversive and appetitive learning in Drosophila. J. Neurosci. 27, 7640-7647.

Kitamoto, T. (2001). Conditional modification of behavior in Drosophila by targeted expression of a temperature-sensitive shibire allele in defined neurons. J. Neurobiol. 47, 81-92.

Knapek, S., Kahsai, L., Winther, A.M.E., Tanimoto, H., and Nassel, D.R. (2013). Short neuropeptide $F$ acts as a functional neuromodulator for olfactory memory in Kenyon cells of Drosophila mushroom bodies. J. Neurosci. 33, 5340-5345.

Koon, A.C., Ashley, J., Barria, R., DasGupta, S., Brain, R., Waddell, S., Alkema, M.J., and Budnik, V. (2011). Autoregulatory and paracrine control of synaptic and behavioral plasticity by octopaminergic signaling. Nat. Neurosci. 14, 190-199.

Kozorovitskiy, Y., Gross, C.G., Kopil, C., Battaglia, L., McBreen, M., Stranahan, A.M., and Gould, E. (2005). Experience induces structural and biochemical changes in the adult primate brain. Proc. Natl. Acad. Sci. U. S. A. 102, 17478-17482.

Krashes, M.J., Keene, A.C., Leung, B., Armstrong, J.D., and Waddell, S. (2007). Sequential use of mushroom body neuron subsets during Drosophila odor memory processing. Neuron 53, 103-115.

Kunz, T., Kraft, K.F., Technau, G.M., and Urbach, R. (2012). Origin of Drosophila mushroom body neuroblasts and generation of divergent embryonic lineages. Development 139, 2510-2522.

Kurusu, M., Awasaki, T., Masuda-Nakagawa, L.M., Kawauchi, H., Ito, K., and Furukubo-Tokunaga, K. (2002). Embryonic and larval development of the Drosophila mushroom bodies: concentric layer subdivisions and the role of fasciclin II. Development 129, 409-419. 
Lai, S.-L., and Lee, T. (2006). Genetic mosaic with dual binary transcriptional systems in Drosophila. Nat. Neurosci. 9, 703-709.

Lamprecht, R., and LeDoux, J. (2004). Structural plasticity and memory. Nat. Rev. Neurosci. 5, 45-54.

Larsson, M.C., Domingos, A.I., Jones, W.D., Chiappe, M.E., Amrein, H., and Vosshall, L.B. (2004). Or83b encodes a broadly expressed odorant receptor essential for Drosophila olfaction. Neuron 43, 703-714.

Lee, D. (2015). Global and local missions of CAMP signaling in neural plasticity, learning, and memory. Front. Pharmacol. 6.

Lee, T., and Luo, L. (1999). Mosaic analysis with a repressible cell marker for studies of gene function in neuronal morphogenesis. Neuron 22, 451-461.

Lee, T., and Luo, L. (2001). Mosaic analysis with a repressible cell marker (MARCM) for Drosophila neural development. Trends Neurosci. 24, 251-254.

Lee, K.-S., You, K.-H., Choo, J.-K., Han, Y.-M., and Yu, K. (2004). Drosophila short neuropeptide $F$ regulates food intake and body size. J. Biol. Chem. 279, 50781-50789.

Lee, P.-T., Lin, H.-W., Chang, Y.-H., Fu, T.-F., Dubnau, J., Hirsh, J., Lee, T., and Chiang, A.-S. (2011). Serotonin-mushroom body circuit modulating the formation of anesthesia-resistant memory in Drosophila. Proc. Natl. Acad. Sci. U. S. A. 108, 1379413799.

Lee, T., Lee, A., and Luo, L. (1999). Development of the Drosophila mushroom bodies: sequential generation of three distinct types of neurons from a neuroblast. Development 126, 4065-4076.

Lee, T., Marticke, S., Sung, C., Robinow, S., and Luo, L. (2000). Cell-autonomous requirement of the USP/ECR-B ecdysone receptor for mushroom body neuronal remodeling in Drosophila. Neuron 28, 807-818.

Leiss, F., Groh, C., Butcher, N.J., Meinertzhagen, I.A., and Tavosanis, G. (2009). Synaptic organization in the adult Drosophila mushroom body calyx. J. Comp. Neurol. $517,808-824$.

Levin, L.R., Han, P.L., Hwang, P.M., Feinstein, P.G., Davis, R.L., and Reed, R.R. (1992). The Drosophila learning and memory gene rutabaga encodes a Ca2+/Calmodulinresponsive adenylyl cyclase. Cell 68, 479-489.

Lin, A.C., Bygrave, A.M., de Calignon, A., Lee, T., and Miesenböck, G. (2014a). Sparse, decorrelated odor coding in the mushroom body enhances learned odor discrimination. Nat. Neurosci. 17, 559-568.

Lin, H.-H., Lai, J.S.-Y., Chin, A.-L., Chen, Y.-C., and Chiang, A.-S. (2007). A map of olfactory representation in the Drosophila mushroom body. Cell 128, 1205-1217. 
Lin, S., Marin, E.C., Yang, C.-P., Kao, C.-F., Apenteng, B.A., Huang, Y., O'Connor, M.B., Truman, J.W., and Lee, T. (2013). Extremes of lineage plasticity in the Drosophila brain. Curr. Biol. 23, 1908-1913.

Lin, S., Owald, D., Chandra, V., Talbot, C., Huetteroth, W., and Waddell, S. (2014b). Neural correlates of water reward in thirsty Drosophila. Nat. Neurosci. 17, 1536-1542.

Lissandron, V., Rossetto, M.G., Erbguth, K., Fiala, A., Daga, A., and Zaccolo, M. (2007). Transgenic fruit-flies expressing a FRET-based sensor for in vivo imaging of cAMP dynamics. Cell. Signal. 19, 2296-2303.

Liu, X., and Davis, R.L. (2009). The GABAergic anterior paired lateral neuron suppresses and is suppressed by olfactory learning. Nat. Neurosci. 12, 53-59.

Liu, C., Plaçais, P.-Y., Yamagata, N., Pfeiffer, B.D., Aso, Y., Friedrich, A.B., Siwanowicz, I., Rubin, G.M., Preat, T., and Tanimoto, H. (2012). A subset of dopamine neurons signals reward for odour memory in Drosophila. Nature 488, 512-516.

Liu, X., Krause, W.C., and Davis, R.L. (2007). GABAA receptor RDL inhibits Drosophila olfactory associative learning. Neuron 56, 1090-1102.

Livingstone, M.S., Sziber, P.P., and Quinn, W.G. (1984). Loss of calcium/calmodulin responsiveness in adenylate cyclase of rutabaga, a Drosophila learning mutant. Cell $37,205-215$.

Luan, H., Peabody, N.C., Vinson, C.R., and White, B.H. (2006). Refined spatial manipulation of neuronal function by combinatorial restriction of transgene expression. Neuron 52, 425-436.

Luo, L., Callaway, E.M., and Svoboda, K. (2008). Genetic dissection of neural circuits. Neuron 57, 634-660.

Ma, J., and Ptashne, M. (1987). The carboxy-terminal 30 amino acids of GAL4 are recognized by GAL80. Cell 50, 137-142.

Mainen, Z.F., and Sejnowski, T.J. (1996). Influence of dendritic structure on firing pattern in model neocortical neurons. Nature 382, 363-366.

Manoli, D.S., Foss, M., Villella, A., Taylor, B.J., Hall, J.C., and Baker, B.S. (2005). Malespecific fruitless specifies the neural substrates of Drosophila courtship behaviour. Nature 436, 395-400.

Mao, Z., and Davis, R.L. (2009). Eight different types of dopaminergic neurons innervate the Drosophila mushroom body neuropil: anatomical and physiological heterogeneity. Front. Neural Circuits 3, 5.

Mao, Z., Roman, G., Zong, L., and Davis, R.L. (2004). Pharmacogenetic rescue in time and space of the rutabaga memory impairment by using Gene-Switch. Proc. Natl. Acad. Sci. U. S. A. 101, 198-203. 
Margulies, C., Tully, T., and Dubnau, J. (2005). Deconstructing memory in Drosophila. Curr. Biol. 15, R700-R713.

Marin, E.C., Jefferis, G.S., Komiyama, T., Zhu, H., and Luo, L. (2002). Representation of the glomerular olfactory map in the Drosophila brain. Cell 109, 243-255.

Martelli, C., Carlson, J.R., and Emonet, T. (2013). Intensity invariant dynamics and odor-specific latencies in olfactory receptor neuron response. J. Neurosci. 33, 62856297.

Masek, P., and Scott, K. (2010). Limited taste discrimination in Drosophila. Proc. Natl. Acad. Sci. U. S. A. 107, 14833-14838.

Masse, N.Y., Turner, G.C., and Jefferis, G.S.X.E. (2009). Olfactory information processing in Drosophila. Curr. Biol. 19, R700-R713.

Mayford, M., Siegelbaum, S.A., and Kandel, E.R. (2012). Synapses and memory storage. Cold Spring Harb. Perspect. Biol. 4, a005751.

McGuire, S.E. (2001). The role of Drosophila mushroom body signaling in olfactory memory. Science 293, 1330-1333.

McGuire, S.E., Le, P.T., Osborn, A.J., Matsumoto, K., and Davis, R.L. (2003). Spatiotemporal rescue of memory dysfunction in Drosophila. Science 302, 1765-1768.

Médioni, J., and Vaysse, G. (1975). Conditional suppression of a reflex in Drosophila melanogaster: acquisition and extinction. C. R. Séances Soc. Biol. Fil. 169, 1386-1391.

Miesenböck, G., De Angelis, D.A., and Rothman, J.E. (1998). Visualizing secretion and synaptic transmission with $\mathrm{pH}$-sensitive green fluorescent proteins. Nature 394, 192195.

Mobbs, P.G. (1982). The brain of the honeybee Apis mellifera. I. The connections and spatial organization of the mushroom bodies. Philos. Trans. R. Soc. Lond. B 298, 309354.

Monastirioti, M., Linn, C.E., and White, K. (1996). Characterization of Drosophila tyramine beta-hydroxylase gene and isolation of mutant flies lacking octopamine. J. Neurosci. 16, 3900-3911.

Murtagh, F., and Contreras, P. (2012). Algorithms for hierarchical clustering: an overview. WIREs Data Min. Knowl. Discov. 2, 86-97.

Murtagh, F., and Legendre, P. (2014). Ward's hierarchical agglomerative clustering method: which algorithms implement Ward's criterion? J. Classif. 31, 274-295.

Murthy, M., Fiete, I., and Laurent, G. (2008). Testing odor response stereotypy in the Drosophila mushroom cody. Neuron 59, 1009-1023. 
Nagel, G., Szellas, T., Huhn, W., Kateriya, S., Adeishvili, N., Berthold, P., Ollig, D., Hegemann, P., and Bamberg, E. (2003). Channelrhodopsin-2, a directly light-gated cation-selective membrane channel. Proc. Natl. Acad. Sci. U. S. A. 100, 13940-13945.

Nighorn, A., Healy, M.J., and Davis, R.L. (1991). The cyclic AMP phosphodiesterase encoded by the Drosophila dunce gene is concentrated in the mushroom body neuropil. Neuron 6, 455-467.

Nikolaev, V.O., Bünemann, M., Hein, L., Hannawacker, A., and Lohse, M.J. (2004). Novel single chain cAMP sensors for receptor-induced signal propagation. J. Biol. Chem. 279, 37215-37218.

Ofstad, T.A., Zuker, C.S., and Reiser, M.B. (2011). Visual place learning in Drosophila melanogaster. Nature 474, 204-207.

O'Kane, C.J., and Gehring, W.J. (1987). Detection in situ of genomic regulatory elements in Drosophila. Proc. Natl. Acad. Sci. U. S. A. 84, 9123-9127.

Olsen, S.R., and Wilson, R.I. (2008). Lateral presynaptic inhibition mediates gain control in an olfactory circuit. Nature 452, 956-960.

Olshausen, B., and Field, D. (2004). Sparse coding of sensory inputs. Curr. Opin. Neurobiol. 14, 481-487.

Owald, D., and Waddell, S. (2015). Olfactory learning skews mushroom body output pathways to steer behavioral choice in Drosophila. Curr. Opin. Neurobiol. 35, 178184.

Owald, D., Felsenberg, J., Talbot, C.B., Das, G., Perisse, E., Huetteroth, W., and Waddell, S. (2015). Activity of defined mushroom body output neurons underlies learned olfactory behavior in Drosophila. Neuron 86, 417-427.

Pai, T.-P., Chen, C.-C., Lin, H.-H., Chin, A.-L., Lai, J.S.-Y., Lee, P.-T., Tully, T., and Chiang, A.-S. (2013). Drosophila ORB protein in two mushroom body output neurons is necessary for long-term memory formation. Proc. Natl. Acad. Sci. U. S. A. 110, 7898-7903.

Pascual, A., and Preat, T. (2001). Localization of long-term memory within the Drosophila mushroom body. Science 294, 1115-1117.

Patterson, M.M., Cegavske, C.F., and Thompson, R.F. (1973). Spinal conditioning. Bull. Psychon. Soc. 1, 139-140.

Pavlov, I.P. (1906). The scientific investigation of the psychical faculties or processes in the higher animals. Science 24, 613-619.

Pavot, P., Carbognin, E., and Martin, J.-R. (2015). PKA and cAMP/CNG channels independently regulate the cholinergic $\mathrm{Ca} 2+$-response of Drosophila mushroom body neurons. eNeuro 2, e0054-14. 
Pech, U., Dipt, S., Barth, J., Singh, P., Jauch, M., Thum, A.S., Fiala, A., and Riemensperger, T. (2013a). Mushroom body miscellanea: transgenic Drosophila strains expressing anatomical and physiological sensor proteins in Kenyon cells. Front. Neural Circuits 7, 147.

Pech, U., Pooryasin, A., Birman, S., and Fiala, A. (2013b). Localization of the contacts between Kenyon cells and aminergic neurons in the Drosophila melanogaster brain using splitGFP reconstitution: splitGFP in Drosophila Mushroom Bodies. J. Comp. Neurol. 521, 3992-4026.

Peng, H., Chung, P., Long, F., Qu, L., Jenett, A., Seeds, A.M., Myers, E.W., and Simpson, J.H. (2011). BrainAligner: 3D registration atlases of Drosophila brains. Nat. Methods 8, 493-498.

Perez-Orive, J. (2002). Oscillations and sparsening of odor representations in the mushroom body. Science 297, 359-365.

Perisse, E., Burke, C., Huetteroth, W., and Waddell, S. (2013a). Shocking revelations and saccharin sweetness in the study of Drosophila olfactory memory. Curr. Biol. 23, R752-R763.

Perisse, E., Yin, Y., Lin, A.C., Lin, S., Huetteroth, W., and Waddell, S. (2013b). Different Kenyon cell populations drive learned approach and avoidance in Drosophila. Neuron 79, 945-956.

Perisse, E., Owald, D., Barnstedt, O., Talbot, C.B., Huetteroth, W., and Waddell, S. (2016). Aversive learning and appetitive motivation toggle feed-forward inhibition in the Drosophila mushroom body. Neuron 90, 1086-1099.

Petilla Interneuron Nomenclature Group, Ascoli, G.A., Alonso-Nanclares, L., Anderson, S.A., Barrionuevo, G., Benavides-Piccione, R., Burkhalter, A., Buzsáki, G., Cauli, B., Defelipe, J., et al. (2008). Petilla terminology: nomenclature of features of GABAergic interneurons of the cerebral cortex. Nat. Rev. Neurosci. 9, 557-568.

Pfeiffer, B.D., Ngo, T.-T.B., Hibbard, K.L., Murphy, C., Jenett, A., Truman, J.W., and Rubin, G.M. (2010). Refinement of tools for targeted gene expression in Drosophila. Genetics 186, 735-755.

Pitman, J.L., McGill, J.J., Keegan, K.P., and Allada, R. (2006). A dynamic role for the mushroom bodies in promoting sleep in Drosophila. Nature 441, 753-756.

Pitman, J.L., DasGupta, S., Krashes, M.J., Leung, B., Perrat, P.N., and Waddell, S. (2009). There are many ways to train a fly. Fly (Austin) 3, 3-9.

Plaçais, P.-Y., Trannoy, S., Friedrich, A.B., Tanimoto, H., and Preat, T. (2013). Two pairs of mushroom body efferent neurons are required for appetitive long-term memory retrieval in Drosophila. Cell Rep. 5, 769-780. 
Poo, C., and Isaacson, J.S. (2009). Odor representations in olfactory cortex: "sparse" coding, global inhibition, and oscillations. Neuron 62, 850-861.

Pooryasin, A. (2014). Neuronal circuit dissection in the Drosophila brain: the role of serotonin-releasing neurons in arousal. Georg-August Universität.

Potter, C.J., Tasic, B., Russler, E.V., Liang, L., and Luo, L. (2010). The Q system: a repressible binary system for transgene expression, lineage tracing, and mosaic analysis. Cell 141, 536-548.

Qin, H., Cressy, M., Li, W., Coravos, J.S., Izzi, S.A., and Dubnau, J. (2012). Gamma neurons mediate dopaminergic input during aversive olfactory memory formation in Drosophila. Curr. Biol. 22, 608-614.

Quinn, W.G., and Dudai, Y. (1976). Memory phases in Drosophila. Nature 262, 576577.

Quinn, W.G., Harris, W.A., and Benzer, S. (1974). Conditioned behavior in Drosophila melanogaster. Proc. Natl. Acad. Sci. U. S. A. 71, 708-712.

Redondo, R.L., and Morris, R.G.M. (2011). Making memories last: the synaptic tagging and capture hypothesis. Nat. Rev. Neurosci. 12, 17-30.

Riemensperger, T., Völler, T., Stock, P., Buchner, E., and Fiala, A. (2005). Punishment prediction by dopaminergic neurons in Drosophila. Curr. Biol. 15, 1953-1960.

Robertson, H.M., Warr, C.G., and Carlson, J.R. (2003). Molecular evolution of the insect chemoreceptor gene superfamily in Drosophila melanogaster. Proc. Natl. Acad. Sci. U. S. A. 100, 14537-14542.

Robinson, G.E. (1992). Regulation of division of labor in insect societies. Annu. Rev. Entomol. 37, 637-665.

Roman, G., and Davis, R.L. (2001). Molecular biology and anatomy of Drosophila olfactory associative learning. BioEssays 23, 571-581.

Root, C.M., Masuyama, K., Green, D.S., Enell, L.E., Nässel, D.R., Lee, C.-H., and Wang, J.W. (2008). A presynaptic gain control mechanism fine-tunes olfactory behavior. Neuron 59, 311-321.

Root, C.M., Ko, K.I., Jafari, A., and Wang, J.W. (2011). Presynaptic facilitation by neuropeptide signaling mediates odor-driven food search. Cell 145, 133-144.

Rousseeuw, P.J. (1987). Silhouettes: a graphical aid to the interpretation and validation of cluster analysis. J. Comput. Appl. Math. 20, 53-65.

Ruta, V., Datta, S.R., Vasconcelos, M.L., Freeland, J., Looger, L.L., and Axel, R. (2010). A dimorphic pheromone circuit in Drosophila from sensory input to descending output. Nature 468, 686-690. 
Scheunemann, L., Jost, E., Richlitzki, A., Day, J.P., Sebastian, S., Thum, A.S., Efetova, M., Davies, S.-A., and Schwarzel, M. (2012). Consolidated and labile odor memory are separately encoded within the Drosophila brain. J. Neurosci. 32, 17163-17171.

Schindelin, J., Arganda-Carreras, I., Frise, E., Kaynig, V., Longair, M., Pietzsch, T., Preibisch, S., Rueden, C., Saalfeld, S., Schmid, B., et al. (2012). Fiji: an open-source platform for biological-image analysis. Nat. Methods 9, 676-682.

Schnaitmann, C., Garbers, C., Wachtler, T., and Tanimoto, H. (2013). Color discrimination with broadband photoreceptors. Curr. Biol. 23, 2375-2382.

Schreurs, B.G. (1989). Classical conditioning of model systems: A behavioral review. Psychobiology 17, 145-155.

Schroll, C., Riemensperger, T., Bucher, D., Ehmer, J., Völler, T., Erbguth, K., Gerber, B., Hendel, T., Nagel, G., Buchner, E., et al. (2006). Light-induced activation of distinct modulatory neurons triggers appetitive or aversive learning in Drosophila larvae. Curr. Biol. 16, 1741-1747.

Schulz, R.A., Chromey, C., Lu, M.F., Zhao, B., and Olson, E.N. (1996). Expression of the D-MEF2 transcription in the Drosophila brain suggests a role in neuronal cell differentiation. Oncogene 12, 1827-1831.

Schwaerzel, M., Heisenberg, M., and Zars, T. (2002). Extinction antagonizes olfactory memory at the subcellular level. Neuron 35, 951-960.

Schwaerzel, M., Monastirioti, M., Scholz, H., Friggi-Grelin, F., Birman, S., and Heisenberg, M. (2003). Dopamine and octopamine differentiate between aversive and appetitive olfactory memories in Drosophila. J. Neurosci. 23, 10495-10502.

Scott, E.K., Raabe, T., and Luo, L. (2002). Structure of the vertical and horizontal system neurons of the lobula plate in Drosophila. J. Comp. Neurol. 454, 470-481.

Séjourné, J., Plaçais, P.-Y., Aso, Y., Siwanowicz, I., Trannoy, S., Thoma, V., Tedjakumala, S.R., Rubin, G.M., Tchénio, P., Ito, K., et al. (2011). Mushroom body efferent neurons responsible for aversive olfactory memory retrieval in Drosophila. Nat. Neurosci. 14, 903-910.

Semmelhack, J.L., and Wang, J.W. (2009). Select Drosophila glomeruli mediate innate olfactory attraction and aversion. Nature 459, 218-223.

Shafer, O.T., Kim, D.J., Dunbar-Yaffe, R., Nikolaev, V.O., Lohse, M.J., and Taghert, P.H. (2008). Widespread receptivity to neuropeptide PDF throughout the neuronal circadian clock network of Drosophila revealed by real-time cyclic AMP imaging. Neuron 58, 223-237.

Shanbhag, S.R., Müller, B., and Steinbrecht, R.A. (1999). Atlas of olfactory organs of Drosophila melanogaster: 1 . Types, external organization, innervation and distribution of olfactory sensilla. Int. J. Insect Morphol. Embryol. 28, 377-397. 
Shang, Y., Claridge-Chang, A., Sjulson, L., Pypaert, M., and Miesenböck, G. (2007). Excitatory local circuits and their implications for olfactory processing in the fly antennal lobe. Cell 128, 601-612.

Shearin, H.K., Macdonald, I.S., Spector, L.P., and Stowers, R.S. (2014). Hexameric GFP and mCherry reporters for the Drosophila GAL4, Q, and LexA transcription systems. Genetics 196, 951-960.

Siegel, R.W., and Hall, J.C. (1979). Conditioned responses in courtship behavior of normal and mutant Drosophila. Proc. Natl. Acad. Sci. U. S. A. 76, 3430-3434.

Sigrist, S.J., and Schmitz, D. (2011). Structural and functional plasticity of the cytoplasmic active zone. Curr. Opin. Neurobiol. 21, 144-150.

Sinakevitch, I., Farris, S.M., and Strausfeld, N.J. (2001). Taurine-, aspartate- and glutamate-like immunoreactivity identifies chemically distinct subdivisions of Kenyon cells in the cockroach mushroom body. J. Comp. Neurol. 439, 352-367.

Sitaraman, D., Aso, Y., Jin, X., Chen, N., Felix, M., Rubin, G.M., and Nitabach, M.N. (2015). Propagation of homeostatic sleep signals by segregated synaptic microcircuits of the Drosophila mushroom body. Curr. Biol. 25, 2915-2927.

Skoulakis, E.M., Kalderon, D., and Davis, R.L. (1993). Preferential expression in mushroom bodies of the catalytic subunit of protein kinase $A$ and its role in learning and memory. Neuron 11, 197-208.

Sokolowski, M.B. (2001). Drosophila: genetics meets behaviour. Nat. Rev. Genet. 2, 879-890.

Stettler, D.D., and Axel, R. (2009). Representations of odor in the piriform cortex. Neuron 63, 854-864.

Stocker, R.F., Lienhard, M.C., Borst, A., and Fischbach, K.F. (1990). Neuronal architecture of the antennal lobe in Drosophila melanogaster. Cell Tissue Res. 262, 934.

Stopfer, M., Jayaraman, V., and Laurent, G. (2003). Intensity versus identity coding in an olfactory system. Neuron 39, 991-1004.

Strausfeld, N.J., Hansen, L., Li, Y., Gomez, R.S., and Ito, K. (1998). Evolution, discovery, and interpretations of arthropod mushroom bodies. Learn. Mem. 5, 11-37.

Strausfeld, N.J., Sinakevitch, I., and Vilinsky, I. (2003). The mushroom bodies of Drosophila melanogaster: An immunocytological and golgi study of Kenyon cell organization in the calyces and lobes. Microsc. Res. Tech. 62, 151-169.

Struhl, G., and Basler, K. (1993). Organizing activity of wingless protein in Drosophila. Cell 72, 527-540. 
Tanaka, N.K., Tanimoto, H., and Ito, K. (2008). Neuronal assemblies of the Drosophila mushroom body. J. Comp. Neurol. 508, 711-755.

Tang, S., and Guo, A. (2001). Choice behavior of Drosophila facing contradictory visual cues. Science 294, 1543-1547.

Technau, G.M. (1984). Fiber number in the mushroom bodies of adult Drosophila melanogaster depends on age, sex and experience. J. Neurogenet. 1, 113-126.

Technau, G., and Heisenberg, M. (1982). Neural reorganization during metamorphosis of the corpora pedunculata in Drosophila melanogaster. Nature 295, 405-407.

Tempel, B.L., Bonini, N., Dawson, D.R., and Quinn, W.G. (1983). Reward learning in normal and mutant Drosophila. Proc. Natl. Acad. Sci. 80, 1482-1486.

Tessier, C.R., and Broadie, K. (2008). Drosophila fragile X mental retardation protein developmentally regulates activity-dependent axon pruning. Development $135,1547-$ 1557.

Thompson, R.F. (1986). The neurobiology of learning and memory. Science 233, 941947.

Thorndike, E.L. (1898). Animal intelligence: An experimental study of the associative processes in animals. Psychol. Rev. 2, 4.

Tian, L., Hires, S.A., Mao, T., Huber, D., Chiappe, M.E., Chalasani, S.H., Petreanu, L., Akerboom, J., McKinney, S.A., Schreiter, E.R., et al. (2009). Imaging neural activity in worms, flies and mice with improved GCaMP calcium indicators. Nat. Methods 6, $875-881$.

Tomchik, S.M., and Davis, R.L. (2009). Dynamics of learning-related cAMP signaling and stimulus integration in the Drosophila olfactory pathway. Neuron 64, 510-521.

Tononi, G., and Cirelli, C. (2014). Sleep and the price of plasticity: from synaptic and cellular homeostasis to memory consolidation and integration. Neuron 81, 12-34.

Tsien, R.Y. (1998). The green fluorescent protein. Annu. Rev. Biochem. 67, 509-544.

Tully, T., and Quinn, W.G. (1985). Classical conditioning and retention in normal and mutant Drosophila melanogaster. J. Comp. Physiol. A 157, 263-277.

Tully, T., Preat, T., Boynton, S.C., and Del Vecchio, M. (1994). Genetic dissection of consolidated memory in Drosophila. Cell 79, 35-47.

Turner, G.C., Bazhenov, M., and Laurent, G. (2008). Olfactory representations by Drosophila mushroom body neurons. J. Neurophysiol. 99, 734-746.

del Valle Rodríguez, A., Didiano, D., and Desplan, C. (2012). Power tools for gene expression and clonal analysis in Drosophila. Nat. Methods 9, 47-55. 
Vasin, A., Zueva, L., Torrez, C., Volfson, D., Littleton, J.T., and Bykhovskaia, M. (2014). Synapsin regulates activity-dependent outgrowth of synaptic boutons at the Drosophila neuromuscular junction. J. Neurosci. 34, 10554-10563.

Vasmer, D., Pooryasin, A., Riemensperger, T., and Fiala, A. (2014). Induction of aversive learning through thermogenetic activation of Kenyon cell ensembles in Drosophila. Front. Behav. Neurosci. 8, 174.

Venken, K.J.T., Simpson, J.H., and Bellen, H.J. (2011). Genetic manipulation of genes and cells in the nervous system of the fruit fly. Neuron 72, 202-230.

Verleyen, P., Huybrechts, J., Baggerman, G., Van Lommel, A., De Loof, A., and Schoofs, L. (2004). SIFamide is a highly conserved neuropeptide: a comparative study in different insect species. Biochem. Biophys. Res. Commun. 320, 334-341.

Vogt, K., Aso, Y., Hige, T., Knapek, S., Ichinose, T., Friedrich, A.B., Turner, G.C., Rubin, G.M., and Tanimoto, H. (2016). Direct neural pathways convey distinct visual information to Drosophila mushroom bodies. eLife 5, e14009.

Von Ohlen, T., and Hooper, J.E. (1999). The ciD mutation encodes a chimeric protein whose activity is regulated by wingless signaling. Dev. Biol. 208, 147-156.

Vosshall, L.B., Wong, A.M., and Axel, R. (2000). An olfactory sensory map in the fly brain. Cell 102, 147-159.

Waddell, S. (2013). Reinforcement signalling in Drosophila; dopamine does it all after all. Curr. Opin. Neurobiol. 23, 324-329.

Waddell, S., and Quinn, W.G. (2001). Flies, genes, and learning. Annu. Rev. Neurosci. 24, 1283-1309.

Waddell, S., Armstrong, J.D., Kitamoto, T., Kaiser, K., and Quinn, W.G. (2000). The amnesiac gene product is expressed in two neurons in the Drosophila brain that are critical for memory. Cell 103, 805-813.

Wagh, D.A., Rasse, T.M., Asan, E., Hofbauer, A., Schwenkert, I., Dürrbeck, H., Buchner, S., Dabauvalle, M.-C., Schmidt, M., Qin, G., et al. (2006). Bruchpilot, a protein with homology to ELKS/CAST, is required for structural integrity and function of synaptic active zones in Drosophila. Neuron 49, 833-844.

Wang, J.W., Wong, A.M., Flores, J., Vosshall, L.B., and Axel, R. (2003). Two-photon calcium imaging reveals an odor-evoked map of activity in the fly brain. Cell 112, 271282.

Wang, Y., Mamiya, A., Chiang, A. -s., and Zhong, Y. (2008). Imaging of an early memory trace in the Drosophila mushroom body. J. Neurosci. 28, 4368-4376.

Ward, J.H. (1963). Hierarchical grouping to optimize an objective function. J. Am. Stat. Assoc. 58, 236-244. 
Wichmann, C., and Sigrist, S.J. (2010). The active zone T-bar--a plasticity module? J. Neurogenet. 24, 133-145.

Wilson, R.I., and Laurent, G. (2005). Role of GABAergic inhibition in shaping odorevoked spatiotemporal patterns in the Drosophila antennal lobe. J. Neurosci. 25, 9069-9079.

Wilson, C., Pearson, R.K., Bellen, H.J., O’Kane, C.J., Grossniklaus, U., and Gehring, W.J. (1989). P-element-mediated enhancer detection: an efficient method for isolating and characterizing developmentally regulated genes in Drosophila. Genes Dev. 3, 1301-1313.

Wilson, R.I., Turner, G.C., and Laurent, G. (2004). Transformation of olfactory representations in the Drosophila antennal lobe. Science 303, 366-370.

Withers, G.S., Fahrbach, S.E., and Robinson, G.E. (1993). Selective neuroanatomical plasticity and division of labour in the honeybee. Nature 364, 238-240.

Wolf, R., Wittig, T., Liu, L., Wustmann, G., Eyding, D., and Heisenberg, M. (1998). Drosophila mushroom bodies are dispensable for visual, tactile, and motor learning. Learn. Mem. 5, 166-178.

Wolff, G.H., and Strausfeld, N.J. (2015). Genealogical correspondence of mushroom bodies across invertebrate phyla. Curr. Biol. 25, 38-44.

Wong, A.M., Wang, J.W., and Axel, R. (2002). Spatial representation of the glomerular map in the Drosophila protocerebrum. Cell 109, 229-241.

Wu, J.S., and Luo, L. (2006). A protocol for mosaic analysis with a repressible cell marker (MARCM) in Drosophila. Nat. Protoc. 1, 2583-2589.

Xia, S., Liu, L., Feng, C., and Guo, A. (1997). Memory consolidation in Drosophila operant visual learning. Learn. Mem. 4, 205-218.

Xia, S., Miyashita, T., Fu, T.-F., Lin, W.-Y., Wu, C.-L., Pyzocha, L., Lin, I.-R., Saitoe, M., Tully, T., and Chiang, A.-S. (2005). NMDA receptors mediate olfactory learning and memory in Drosophila. Curr. Biol. 15, 603-615.

Xie, Z., Huang, C., Ci, B., Wang, L., and Zhong, Y. (2013). Requirement of the combination of mushroom body $y$ lobe and $a / \beta$ lobes for the retrieval of both aversive and appetitive early memories in Drosophila. Learn. Mem. 20,474-481.

Xu, T., and Rubin, G.M. (1993). Analysis of genetic mosaics in developing and adult Drosophila tissues. Development 117, 1223-1237.

Xu, T., Yu, X., Perlik, A.J., Tobin, W.F., Zweig, J.A., Tennant, K., Jones, T., and Zuo, Y. (2009). Rapid formation and selective stabilization of synapses for enduring motor memories. Nature 462, 915-919. 
Yamagata, N., Ichinose, T., Aso, Y., Plaçais, P.-Y., Friedrich, A.B., Sima, R.J., Preat, T., Rubin, G.M., and Tanimoto, H. (2015). Distinct dopamine neurons mediate reward signals for short- and long-term memories. Proc. Natl. Acad. Sci. 112, 578-583.

Yang, M.Y., Armstrong, J.D., Vilinsky, I., Strausfeld, N.J., and Kaiser, K. (1995). Subdivision of the Drosophila mushroom bodies by enhancer-trap expression patterns. Neuron 15, 45-54.

Yasuyama, K., Meinertzhagen, I.A., and Schürmann, F.-W. (2002). Synaptic organization of the mushroom body calyx in Drosophila melanogaster. J. Comp. Neurol. 445, 211-226.

Yin, J.C., Wallach, J.S., Del Vecchio, M., Wilder, E.L., Zhou, H., Quinn, W.G., and Tully, T. (1994). Induction of a dominant negative CREB transgene specifically blocks longterm memory in Drosophila. Cell 79, 49-58.

Yu, F., and Schuldiner, O. (2014). Axon and dendrite pruning in Drosophila. Curr. Opin. Neurobiol. 27, 192-198.

Yu, D., Keene, A.C., Srivatsan, A., Waddell, S., and Davis, R.L. (2005). Drosophila DPM neurons form a delayed and branch-specific memory trace after olfactory classical conditioning. Cell 123, 945-957.

Yu, D., Akalal, D.-B.G., and Davis, R.L. (2006). Drosophila a/ $\beta$ mushroom body neurons form a branch-specific, long-term cellular memory trace after spaced olfactory conditioning. Neuron 52, 845-855.

Yu, H.-H., Chen, C.-H., Shi, L., Huang, Y., and Lee, T. (2009). Twin-spot MARCM to reveal the developmental origin and identity of neurons. Nat. Neurosci. 12, 947-953.

Yu, H.-H., Awasaki, T., Schroeder, M.D., Long, F., Yang, J.S., He, Y., Ding, P., Kao, J.C., Wu, G.Y.-Y., Peng, H., et al. (2013). Clonal development and organization of the adult Drosophila central brain. Curr. Biol. CB 23, 633-643.

Yu, J.Y., Kanai, M.I., Demir, E., Jefferis, G.S.X.E., and Dickson, B.J. (2010). Cellular Organization of the Neural Circuit that Drives Drosophila Courtship Behavior. Curr. Biol. 20, 1602-1614.

Yuan, Q., Lin, F., Zheng, X., and Sehgal, A. (2005). Serotonin modulates circadian entrainment in Drosophila. Neuron 47, 115-127.

Yuan, Q., Xiang, Y., Yan, Z., Han, C., Jan, L.Y., and Jan, Y.N. (2011). Light-induced structural and functional plasticity in Drosophila larval visual system. Science 333, $1458-1462$.

Zars, T. (2000). Localization of a short-term memory in Drosophila. Science 288, 672675. 
Zhong, Y., Budnik, V., and Wu, C.F. (1992). Synaptic plasticity in Drosophila memory and hyperexcitable mutants: role of cAMP cascade. J. Neurosci. 12, 644-651.

Zhu, S., Chiang, A.-S., and Lee, T. (2003). Development of the Drosophila mushroom bodies: elaboration, remodeling and spatial organization of dendrites in the calyx. Development 130, 2603-2610. 


\section{Abbreviations}

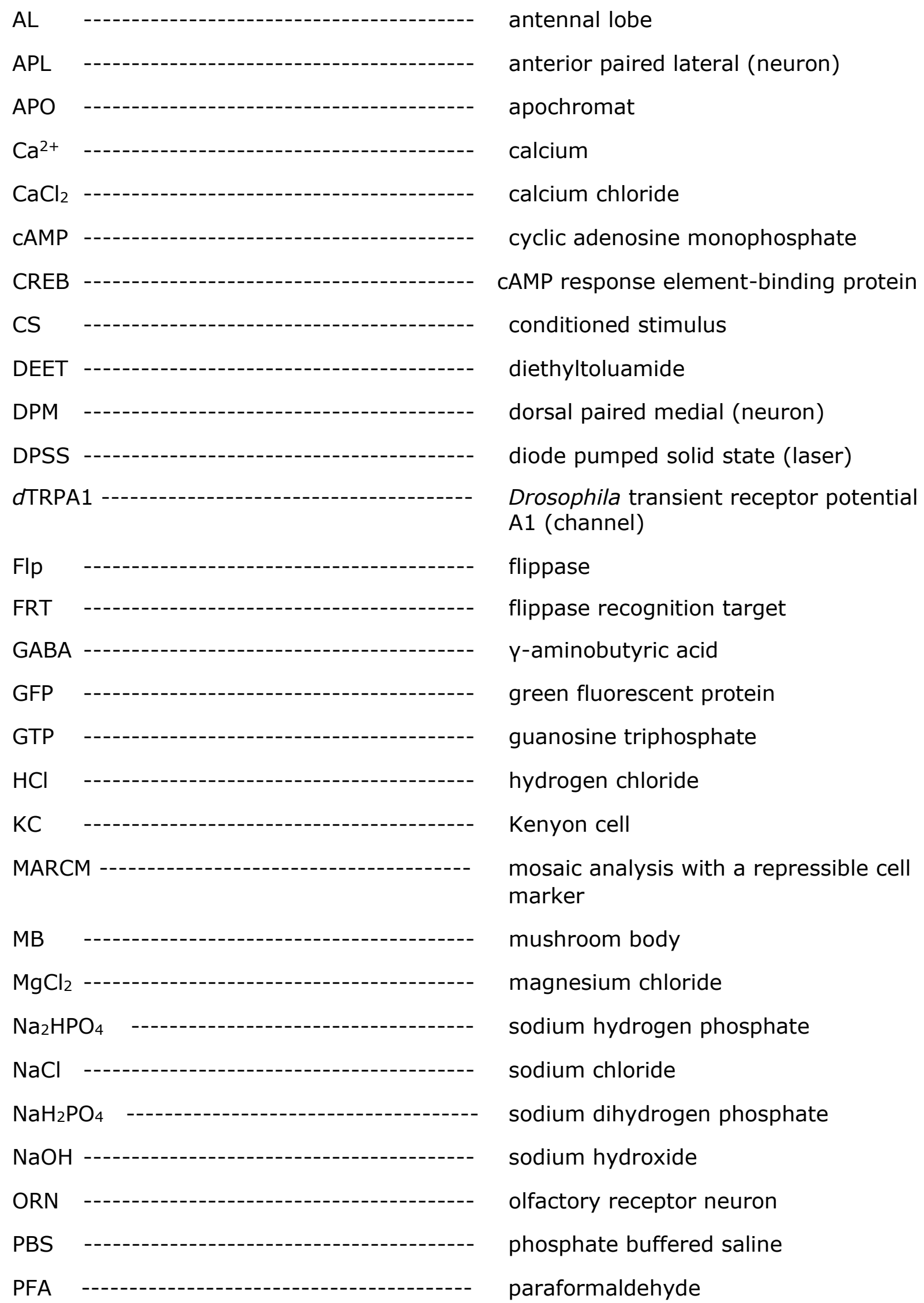




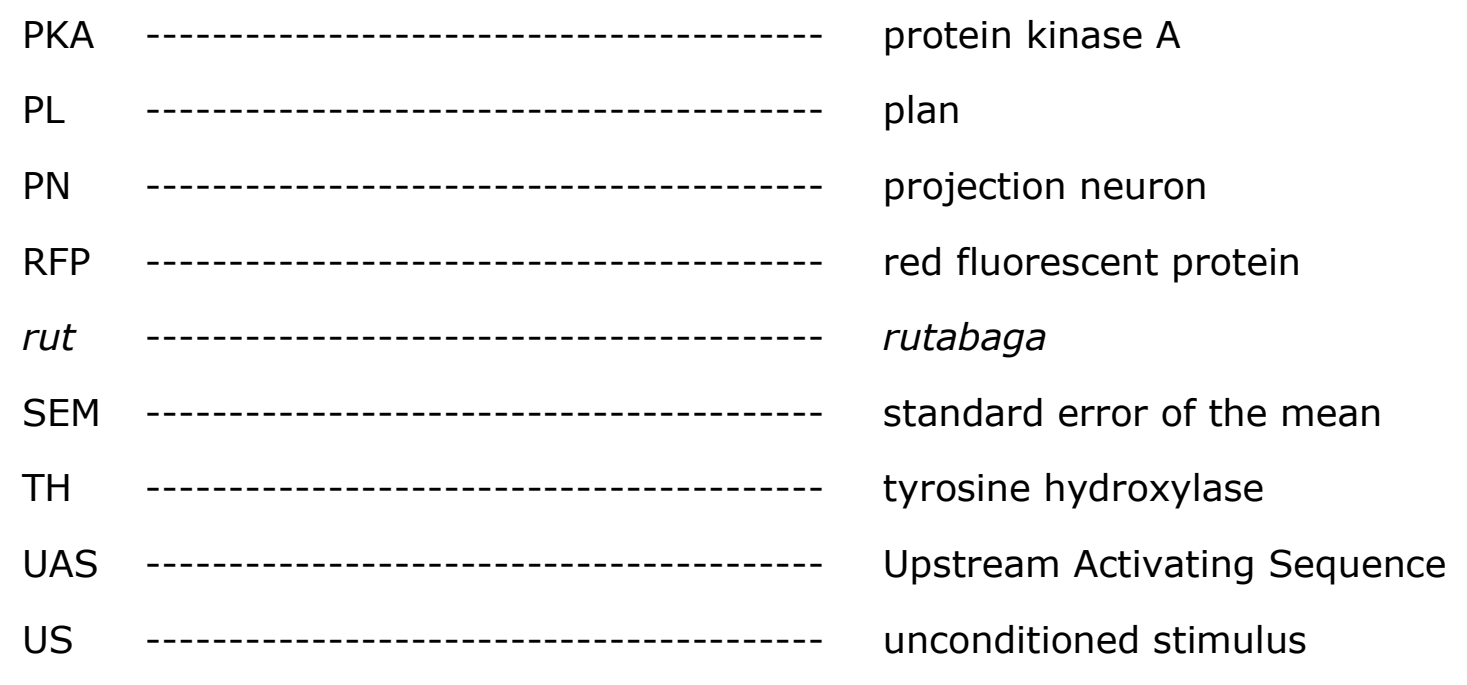




\section{APPENDIX I: R scripts}

Different R scripts have been programmed in order to perform (I) cluster analysis, (II) centroid and nearest neighbor calculations, (III) silhouette value calculations, and (IV) principle component analysis.

\section{(I) Cluster analysis}

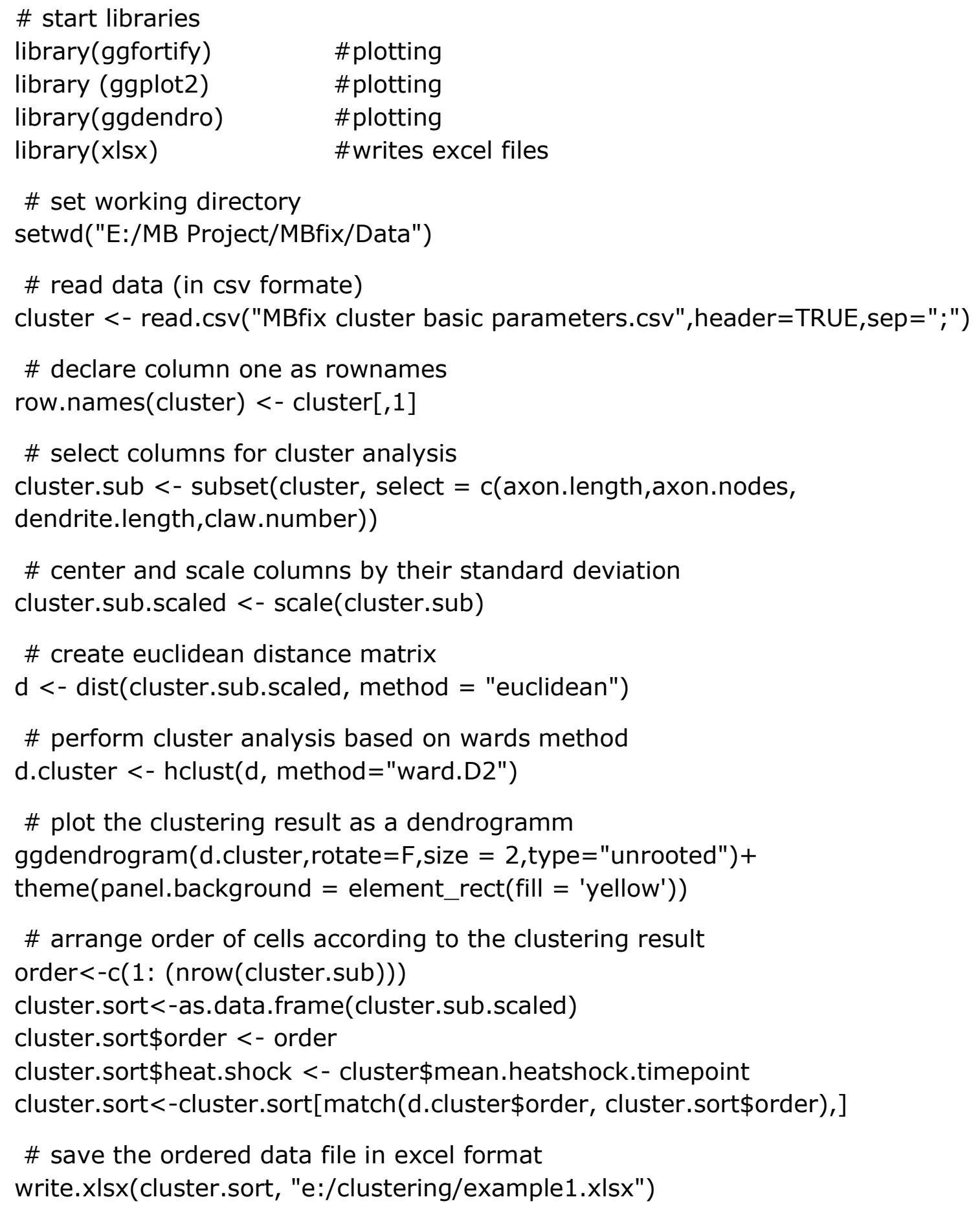




\section{(II) Centroid and nearest neighbor}

\# start libraries

library(xIsx)

\# set working directory

setwd("e:/MB project/MBfix/Clustering \& PCA in R/cluster analysis basic parameters")

\# read data (in csv formate)

cluster <- read.csv("MBfix bp cluster sorted scaled cluster

2.2.csv",header=TRUE, $\operatorname{sep}=" ; ")$

\# declare column one as rownames

row.names(cluster) <- cluster[,1]

\# select columns for centroid analysis

cluster $<-$ subset(cluster, select $=c$ (axon.length,axon.nodes,

dendrite. length, claw.number))

\# calculate median center of the respective cluster

center.median <- c(median(cluster[,1]), median(cluster[,2]),

median(cluster[,3]), median(cluster[,4]))

\# add the median center to the cluster and give it the name "center"

combo <- rbind(cluster, center.median)

rownames(combo)[nrow(cluster)+1] <- "center"

\# create euclidean distance matrix, save "center" column as extra variable

d.combo <- as.matrix(as.matrix(dist(combo)) [nrow(cluster) +1,$]$ )

d.combo $2<-$ d.combo[-(nrow(cluster) +1$)$, ]

\# find nearest neighbor

NN <- as. $\operatorname{matrix}($ which (d.combo2 $==\min ($ d.combo2), arr.ind $=$ TRUE) $)$

$\mathrm{NN}[1]<,-\min ($ d.combo2)

\# save median center in excel format

write.xIsx(center.median,"e:/clustering/MBfix bp cluster 2.2 median center.xlsx")

\section{(III) Silhouette values}

\# start libraries

library(xIsx)

\# set working directory

setwd("E:/MB Project/MBfix/Clustering \& PCA in R/cluster analysis basic parameters")

\# read data of cluster 1 (in csv formate)

cluster1.1 <- read.csv("MBfix bp cluster sorted scaled cluster

1.1.csv",header=TRUE, $\operatorname{sep}=" ; ")$ 
\# read data of cluster 2 (in csv formate)

cluster2.1 <- read.csv("MBfix bp cluster sorted scaled cluster

1.2.csv", header=TRUE, $\operatorname{sep}=" ; ")$

\# declare column one as rownames and delete the first column

row.names(cluster1.1) <- cluster $1.1[, 1]$

cluster1.2 <- cluster1.1[,-c(1)]

row.names(cluster2.1) <- cluster $2.1[, 1]$

cluster2. $2<-$ cluster $2.1[,-c(1)]$

\# create euclidean distance matrices

cluster1.3<-as.matrix(dist(cluster1.2))

cluster2.3<-as.matrix(dist(cluster2.2))

\# create matrix for silhouette values of cluster 1

all. silhouettes <- matrix (nrow=ncol(cluster1.3), ncol=1)

rownames(all.silhouettes) <- rownames(cluster1.3)

colnames(all.silhouettes) <- "silhouette value"

\# loop to calculate silhouette values

for (i in 1:ncol(cluster1.3))

\{

\# a1.3 saves distances of cell $i$ to other cells within its cluster

\# and excludes distance to itself

a1.1 <- cluster1.3 [,c(i)]

a1.2 <- as.matrix (a1.1)

a1.3 <- as.matrix $(\mathrm{a} 1.2[-\mathrm{c}(\mathrm{i})]$,

\# in combo1.1 to combo1.6 the cell $\mathrm{i}$ is added to the neighboring cluster

\# the distance matrix is calculated to show distance of cell $i$ to all other

\# cells in that cluster

combo1.1<-rbind(cluster2.2, cluster1.2[i,])

combo1.2<-dist(combo1.1)

combo1.3<-as.matrix(combo1.2)

combo1.4<-combo1.3[nrow(combo1.1),]

combo1.5<-as.matrix (combo1.4)

combo1.6<-as.matrix(combo1.5[-nrow(combo1.1),])

\# calculates the silhouette of cell $i$

silhouette <- (mean(combo1.6)-mean(a1.3))/max(mean(a1.3), mean(combo1.6))

\# saves the silhouette of cell $i$ in row $i$ of the silhouette matrix

all.silhouettes $[\mathrm{i}]<$,- silhouette

\}

\# save silhouette values of cluster 1 in excel format

write.xIsx(all.silhouettes,"e:/clustering/example silhouette values.xIsx") 


\section{(IV) Principle component analysis}

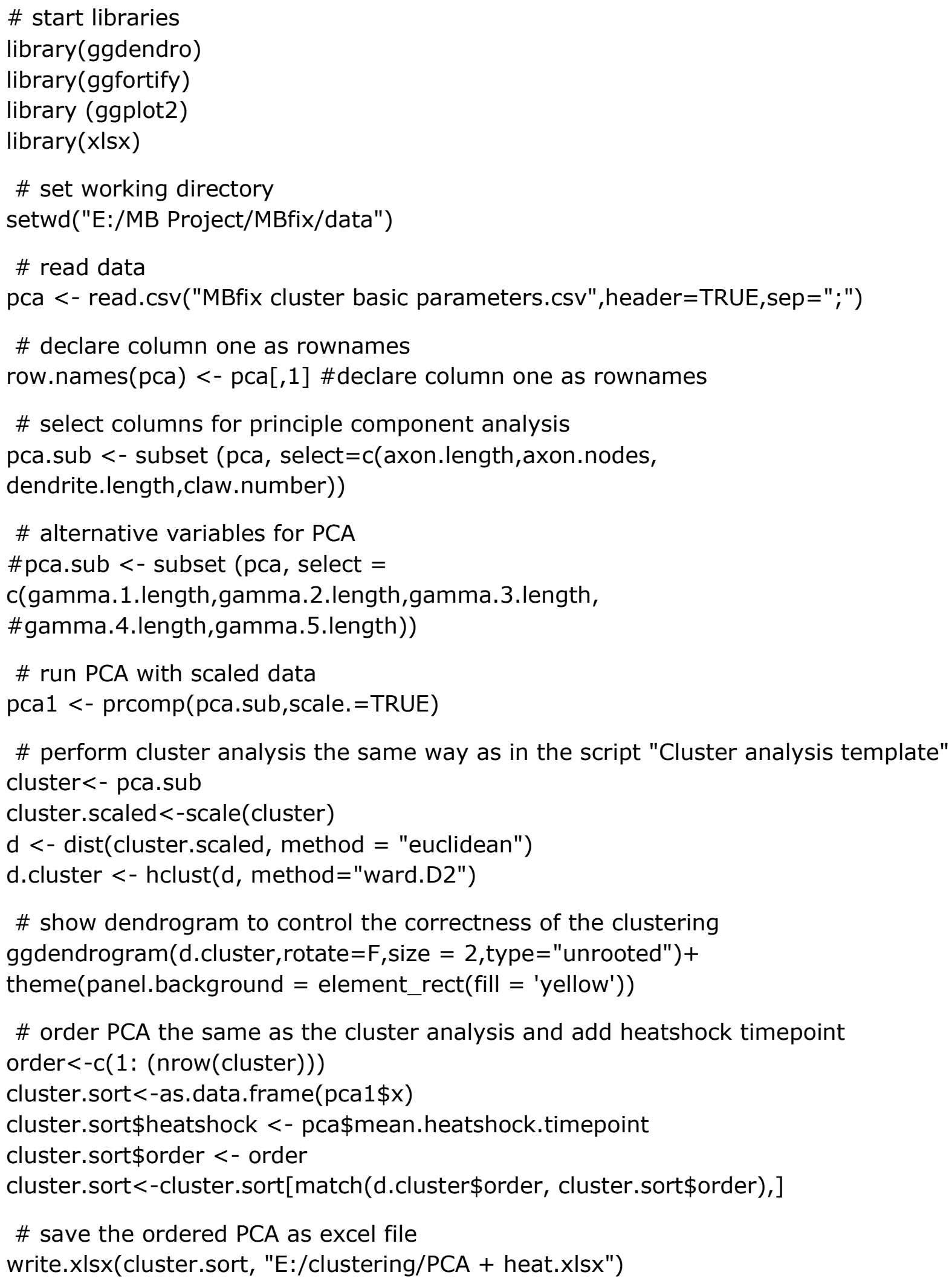




\section{David Vasmer}

Date of birth: February 12, 1986

Citizenship: German

Address: Burgstraße 9, 37073, Göttingen

eMail:d.vasmer@gmail.com

\section{Education}

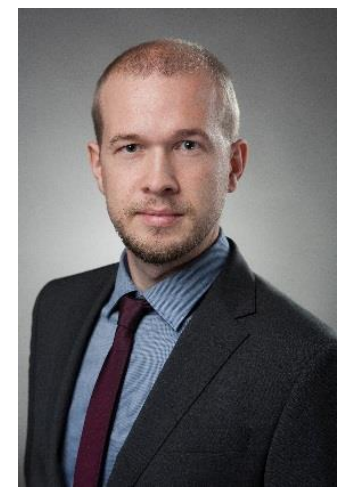

May 2013 - today

University of

Göttingen

Oct 2010 - Apr 2013

University of

Göttingen

Oct 2006 - Sep 2009

University of

Göttingen
Dr. rer. nat. in the deparment of Molecular Neurobiologiy of Behavior, doctoral program: „Sensory and Motor Neuroscience"

Thesis: „Morphological analysis of Kenyon cells of the Drosophila mushroom bodies"

Master of Science in Developmental, Neural, and Behavioral Biology

Thesis: "Thermogenetic induction of learning in Kenyon cells of the Drosophila mushroom bodies"

Bachelor Biology

Thesis: "Leadership in fish shoals"

\section{Awards \& Publications}

* Excellence stipend awarded by the Göttingen Graduate School for Neurosciences, Biophysics, and Molecular (GGNB) for the doctoral project, Jan 2014 - Dec 2016

* Vasmer, D., Pooryasin, A., Riemensperger, T. \& Fiala, A. Induction of aversive learning through thermogenetic activation of Kenyon cell ensembles in Drosophila. (2014) 